\title{
Treatment of periodontal disease for glycaemic control in people with diabetes mellitus (Review)
}

Simpson TC, Weldon JC, Worthington HV, Needleman I, Wild SH, Moles DR, Stevenson B, Furness $S$, Iheozor-Ejiofor Z 
TABLE OF CONTENTS

HEADER

ABSTRACT

PLAIN LANGUAGE SUMMARY

SUMMARY OF FINDINGS

BACKGROUND

OBJECTIVES

METHODS

RESULTS

Figure 1.

Figure 2.

Figure 3.

Figure 4.

Figure 5.

DISCUSSION

AUTHORS' CONCLUSIONS

ACKNOWLEDGEMENTS

REFERENCES

CHARACTERISTICS OF STUDIES

DATA AND ANALYSES

Analysis 1.1. Comparison 1 Periodontal therapy versus no active intervention/usual care, Outcome $1 \mathrm{HbA} 1 \mathrm{c}$ at 3-4 months. ...

Analysis 1.2. Comparison 1 Periodontal therapy versus no active intervention/usual care, Outcome $2 \mathrm{HbA} 1 \mathrm{c}$ at 6 months. .....

Analysis 2.1. Comparison 2 Periodontal therapy versus alternative periodontal therapy, Outcome $1 \mathrm{HbA} 1 \mathrm{c}$ at 3-4 months. .....

Analysis 2.2. Comparison 2 Periodontal therapy versus alternative periodontal therapy, Outcome $2 \mathrm{HbA1c}$ at 6 months. ADDITIONAL TABLES

APPENDICES

WHAT'S NEW

HISTORY

CONTRIBUTIONS OF AUTHORS

DECLARATIONS OF INTEREST

SOURCES OF SUPPORT

DIFFERENCES BETWEEN PROTOCOL AND REVIEW

INDEX TERMS 
[Intervention Review]

\section{Treatment of periodontal disease for glycaemic control in people with diabetes mellitus}

Terry C Simpson ${ }^{1}$, Jo C Weldon ${ }^{2}$, Helen V Worthington 2 , Ian Needleman 3 , Sarah H Wild 4 , David R Moles 5 , Brian Stevenson ${ }^{6}$, Susan Furness ${ }^{2}$, Zipporah Iheozor-Ejiofor ${ }^{7}$

1Edinburgh Dental Institute, University of Edinburgh, Edinburgh, UK. ${ }^{2}$ Cochrane Oral Health, Division of Dentistry, School of Medical Sciences, Faculty of Biology, Medicine and Health, The University of Manchester, Manchester, UK. 3 Unit of Periodontology and International Centre for Evidence-Based Oral Health, UCL Eastman Dental Institute, London, UK. ${ }^{4}$ Centre for Public Health and Primary Care Research, Public Health Sciences, University of Edinburgh, Edinburgh, UK. ${ }^{5}$ Oral Health Services Research, Peninsula Dental School, Plymouth, UK. ${ }^{6}$ Department of Restorative Dentistry, University of Dundee Dental Hospital and School, Dundee, UK. ${ }^{7}$ School of Medicine, University of Central Lancashire, Preston, Lancashire, UK

Contact address: Jo C Weldon, Cochrane Oral Health, Division of Dentistry, School of Medical Sciences, Faculty of Biology, Medicine and Health, The University of Manchester, JR Moore Building, Oxford Road, Manchester, M13 9PL, UK. jo.weldon@manchester.ac.uk.

Editorial group: Cochrane Oral Health Group.

Publication status and date: Edited (no change to conclusions), published in Issue 3, 2018.

Citation: Simpson TC, Weldon JC, Worthington HV, Needleman I, Wild SH, Moles DR, Stevenson B, Furness S, Iheozor-Ejiofor Z. Treatment of periodontal disease for glycaemic control in people with diabetes mellitus. Cochrane Database of Systematic Reviews 2015, Issue 11. Art. No.: CD004714. DOI: 10.1002/14651858.CD004714.pub3.

Copyright $\odot 2018$ The Cochrane Collaboration. Published by John Wiley \& Sons, Ltd.

\section{A B S T R A C T}

\section{Background}

Glycaemic control is a key issue in the care of people with diabetes mellitus (DM). Periodontal disease is the inflammation and destruction of the underlying supporting tissues of the teeth. Some studies have suggested a bidirectional relationship between glycaemic control and periodontal disease. This review updates the previous version published in 2010.

\section{Objectives}

The objective is to investigate the effect of periodontal therapy on glycaemic control in people with diabetes mellitus.

\section{Search methods}

We searched the following electronic databases: the Cochrane Oral Health Group Trials Register (to 31 December 2014), the Cochrane Central Register of Controlled Trials (CENTRAL) (Cochrane Library 2014, Issue 11), MEDLINE via OVID (1946 to 31 December 2014 ), EMBASE via OVID (1980 to 31 December 2014), LILACS via BIREME (1982 to 31 December 2014), and CINAHL via EBSCO (1937 to 31 December 2014). ZETOC (1993 to 31 December 2014) and Web of Knowledge (1990 to 31 December 2014) were searched for conference proceedings. Additionally, two periodontology journals were handsearched for completeness, Annals of Periodontology (1996 to 2003) and Periodontology 2000 (1993 to 2003). We searched the US National Institutes of Health Trials Registry (http://clinicaltrials.gov) and the WHO Clinical Trials Registry Platform for ongoing trials. No restrictions were placed on the language or date of publication when searching the electronic databases.

\section{Selection criteria}

We searched for randomised controlled trials (RCTs) of people with type 1 or type 2 DM (T1DM/T2DM) with a diagnosis of periodontitis. Interventions included periodontal treatments such as mechanical debridement, surgical treatment and antimicrobial therapy. Two broad comparisons were proposed:

1. periodontal therapy versus no active intervention/usual care; 
2. periodontal therapy versus alternative periodontal therapy.

\section{Data collection and analysis}

For this review update, at least two review authors independently examined the titles and abstracts retrieved by the search, selected the included trials, extracted data from included trials and assessed included trials for risk of bias.

Our primary outcome was blood glucose levels measured as glycated (glycosylated) haemoglobin assay (HbA1c).

Our secondary outcomes included adverse effects, periodontal indices (bleeding on probing (BOP), clinical attachment level (CAL), gingival index (GI), plaque index (PI) and probing pocket depth (PPD)), cost implications and diabetic complications.

\section{Main results}

We included 35 studies (including seven from the previous version of the review), which included 2565 participants in total. All studies used a parallel RCT design, and 33 studies (94\%) only targeted T2DM patients. There was variation between studies with regards to included age groups (ages 18 to 80 ), duration of follow-up (3 to 12 months), use of antidiabetic therapy, and included participants' baseline HbA1c levels (from $5.5 \%$ to $13.1 \%$ ).

We assessed 29 studies (83\%) as being at high risk of bias, two studies (6\%) as being at low risk of bias, and four studies (11\%) as unclear. Thirty-four of the studies provided data suitable for analysis under one or both of the two comparisons.

Comparison 1: low quality evidence from 14 studies (1499 participants) comparing periodontal therapy with no active intervention/usual care demonstrated that mean $\mathrm{HbA1c}$ was $0.29 \%$ lower ( $95 \%$ confidence interval $(\mathrm{Cl}) 0.48 \%$ to $0.10 \%$ lower) 3 to 4 months post-treatment, and $0.02 \%$ lower after 6 months (five studies, 826 participants; $95 \% \mathrm{Cl} 0.20 \%$ lower to $0.16 \%$ higher).

Comparison 2: 21 studies (920 participants) compared different periodontal therapies with each other. There was only very low quality evidence for the multiple head-to-head comparisons, the majority of which were unsuitable to be pooled, and provided no clear evidence of a benefit for one periodontal intervention over another. We were able to pool the specific comparison between scaling and root planing (SRP) plus antimicrobial versus SRP and there was no consistent evidence that the addition of antimicrobials to SRP was of any benefit to delivering SRP alone (mean HbA1c $0.00 \%$ lower: 12 studies, 450 participants; $95 \% \mathrm{Cl} 0.22 \%$ lower to $0.22 \%$ higher) at 3-4 months posttreatment, or after 6 months (mean HbA1c 0.04\% lower: five studies, 206 patients; $95 \% \mathrm{Cl} 0.41 \%$ lower to $0.32 \%$ higher).

Less than half of the studies measured adverse effects. The evidence was insufficient to conclude whether any of the treatments were associated with harm. No other patient-reported outcomes (e.g. quality of life) were measured by the included studies, and neither were cost implications or diabetic complications.

Studies showed varying degrees of success with regards to achieving periodontal health, with some showing high levels of residual inflammation following treatment. Statistically significant improvements were shown for all periodontal indices (BOP, CAL, GI, PI and PPD) at 3-4 and 6 months in comparison 1; however, this was less clear for individual comparisons within the broad category of comparison 2.

\section{Authors' conclusions}

There is low quality evidence that the treatment of periodontal disease by SRP does improve glycaemic control in people with diabetes, with a mean percentage reduction in HbAlc of $0.29 \%$ at 3-4 months; however, there is insufficient evidence to demonstrate that this is maintained after 4 months.

There was no evidence to support that one periodontal therapy was more effective than another in improving glycaemic control in people with diabetes mellitus.

In clinical practice, ongoing professional periodontal treatment will be required to maintain clinical improvements beyond 6 months. Further research is required to determine whether adjunctive drug therapies should be used with periodontal treatment. Future RCTs should evaluate this, provide longer follow-up periods, and consider the inclusion of a third 'no treatment' control arm.

Larger, well conducted and clearly reported studies are needed in order to understand the potential of periodontal treatment to improve glycaemic control among people with diabetes mellitus. In addition, it will be important in future studies that the intervention is effective in reducing periodontal inflammation and maintaining it at lowered levels throughout the period of observation.

\section{PLAIN LANGUAGE SUMMARY}

\section{Does treatment for gum disease help people with diabetes control blood sugar levels?}

\section{Review question}

The main question addressed by this review is: how effective is gum disease treatment for controlling blood sugar levels (known as glycaemic control) in people with diabetes, compared to no active treatment or usual care? 


\section{Background}

Gum disease treatment is used to reduce swelling and infection from gum disease. Keeping blood sugar levels under control is a key issue for people with diabetes, and some clinical research suggests a relationship exists between gum disease treatment and glycaemic control. As a result, it is important to discover if gum disease treatment does improve glycaemic control to encourage better use of clinical resources.

There is a broad range of gum disease treatments available for treating patients with diabetes. This review considered two types.

1. Does gum disease treatment improve blood sugar control in people with diabetes?

2. Does one type of gum disease treatment have a bigger effect than another in improving blood sugar control?

\section{Study characteristics}

This review of existing clinical trials was carried out by authors working with the Cochrane Oral Health Group and updates the previous version published in 2010. The evidence is current up to 31 December 2014.

In this review there are 35 trials (including 2565 participants), published between 1997 and 2014, where people randomly received a type of gum disease treatment (including scaling and root planing (SRP) and SRP combined with other types of treatment), or usual care/no active treatment.

The trials included in this review used SRP with, or without, an additional treatment. Additional treatments included instructions for cleaning teeth properly (known as oral hygiene instruction $(\mathrm{OHI})$ ), and other gum treatments (for example, antimicrobials, which are used to treat infections).

\section{Key results}

We found 35 trials that were suitable for inclusion in this review. Thirty-four of those studies provided results that could be included in at least one of the two comparisons.

1. The evidence from 14 trials (1499 participants) showed that SRP reduces blood sugar levels in diabetic patients by $0.29 \%$ up to 4 months after receiving care when compared with usual care/no active treatment. After 6 months, there was no evidence that this reduction was sustained.

2. The evidence from 21 trials (920 participants) investigating different types of gum disease treatments failed to show that one treatment was better than another.

There were not enough studies measuring side effects to be able to show if gum disease treatments cause any harm.

\section{Quality of the evidence}

Currently there is low quality evidence to support using scaling and root planing for controlling blood sugar levels up to 4 months after receiving treatment. Ongoing gum disease treatment is advised to maintain improvements in blood sugar levels. 
SUMMARY OF FINDINGS

Summary of findings for the main comparison. Periodontal therapy compared to no active intervention/usual care for glycaemic control in people with diabetes mellitus

Periodontal therapy versus no active intervention/usual care for glycaemic control in people with diabetes mellitus

Patient or population: Patients with diabetes mellitus

Settings: Hospital, primary care, community

Intervention: Periodontal therapy

Comparison: Usual care/no active treatment

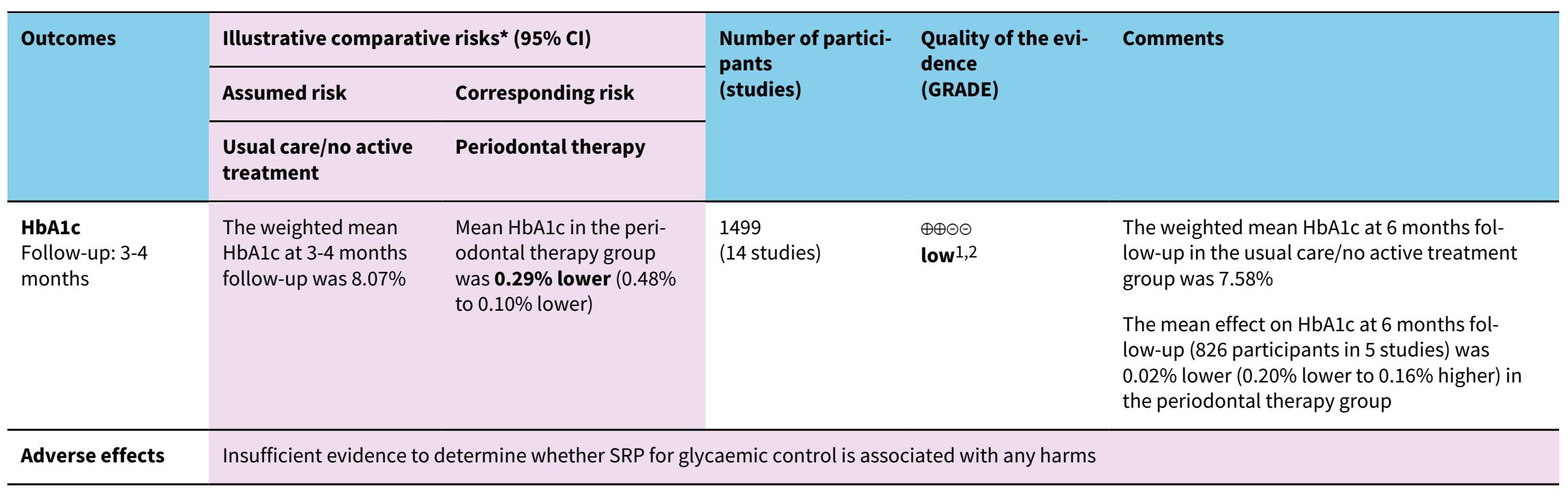

*The basis for the assumed risk (eg the median control group risk across studies) is provided in footnotes. The corresponding risk (and its $95 \% \mathrm{Cl}$ ) is based on the assumed

risk in the comparison group and the relative effect of the intervention (and its $95 \% \mathrm{Cl}$ )

CI: confidence interval; SRP: scaling and root planing

GRADE Working Group grades of evidence

High quality: Further research is very unlikely to change our confidence in the estimate of effect

Moderate quality: Further research is likely to have an important impact on our confidence in the estimate of effect and may change the estimate

Low quality: Further research is very likely to have an important impact on our confidence in the estimate of effect and is likely to change the estimate

Very low quality: We are very uncertain about the estimate

1 High risk of bias, largely due to lack of blinding: quality of evidence downgraded once

2 Moderate heterogeneity $\left(I^{2}=53 \%\right)$ : quality of evidence downgraded once 
Summary of findings 2. Periodontal therapy compared to alternative periodontal therapy for glycaemic control in people with diabetes mellitus

Periodontal therapy versus alternative periodontal therapy for glycaemic control in people with diabetes mellitus

Patient or population: Patients with diabetes mellitus

Settings: Hospital

Intervention: Periodontal therapy ${ }^{1}$

Comparison: Alternative periodontal therapy 1

\begin{tabular}{|c|c|c|c|c|c|c|}
\hline \multirow[t]{3}{*}{ Outcomes } & & \multicolumn{2}{|c|}{ Illustrative comparative risks* $(95 \% \mathrm{CI})$} & \multirow{3}{*}{$\begin{array}{l}\text { Number of par- } \\
\text { ticipants } \\
\text { (studies) }\end{array}$} & \multirow{3}{*}{$\begin{array}{l}\text { Quality of the } \\
\text { evidence } \\
\text { (GRADE) }\end{array}$} & \multirow[t]{3}{*}{ Comments } \\
\hline & & \multirow{2}{*}{$\begin{array}{l}\text { Assumed risk } \\
\begin{array}{l}\text { Alternative periodontal } \\
\text { therapy }\end{array}\end{array}$} & \multirow{2}{*}{\begin{tabular}{|l} 
Corresponding risk \\
Periodontal therapy
\end{tabular}} & & & \\
\hline & & & & & & \\
\hline $\begin{array}{l}\text { HbA1c } \\
\text { Follow-up: 3-4 } \\
\text { months }\end{array}$ & $\begin{array}{l}\text { SRP plus an- } \\
\text { timicrobial } \\
\text { versus SRP }\end{array}$ & $\begin{array}{l}\text { The weighted mean } \\
\text { HbAlc at } 3-4 \text { months fol- } \\
\text { low-up was } 8.04 \% \text { in the } \\
\text { SRP group }\end{array}$ & $\begin{array}{l}\text { Mean HbAlc in the SRP } \\
\text { plus antimicrobial group } \\
\text { was } \mathbf{0 . 0 0 \%} \text { lower }(0.22 \% \\
\text { lower to } 0.22 \% \text { higher) }\end{array}$ & $\begin{array}{l}450 \\
\text { (12 studies) }\end{array}$ & $\begin{array}{l}\oplus \odot \odot \ominus \\
\text { very low } 2,3\end{array}$ & $\begin{array}{l}\text { We were unable to pool the results for } \\
\text { all the other comparisons (apart from } \\
1^{4} \text { ), due to differences in the inter- } \\
\text { ventions being compared. The results } \\
\text { from these small studies (and meta- } \\
\text { analysis) provide no clear evidence of } \\
\text { a benefit }\end{array}$ \\
\hline $\begin{array}{l}\text { Adverse ef- } \\
\text { fects }\end{array}$ & $\begin{array}{l}\text { Insufficient e } \\
\text { nation comp }\end{array}$ & $\begin{array}{l}\text { e to determine whether } m \\
\text { that received by control }\end{array}$ & $\begin{array}{l}\text { re intensive periodontal th } \\
\text { oups) for glycaemic contro }\end{array}$ & $\begin{array}{l}\text { erapy delivery (int } \\
\text { is associated witl }\end{array}$ & $\begin{array}{l}\text { vention groups } \\
\text { ny harms }\end{array}$ & iving an additional treatment combi- \\
\hline
\end{tabular}

*The basis for the assumed risk (eg the median control group risk across studies) is provided in footnotes. The corresponding risk (and its $95 \% \mathrm{Cl}$ ) is based on the assumed risk in the comparison group and the relative effect of the intervention (and its 95\% Cl)

Cl: confidence interval; SRP: scaling and root planing

GRADE Working Group grades of evidence

High quality: Further research is very unlikely to change our confidence in the estimate of effect

Moderate quality: Further research is likely to have an important impact on our confidence in the estimate of effect and may change the estimate

Low quality: Further research is very likely to have an important impact on our confidence in the estimate of effect and is likely to change the estimate

Very low quality: We are very uncertain about the estimate

1 Interventions varied across studies, and are clearly detailed in Additional Table 5 within this review

2 Moderate to high risk of bias across domains: quality of evidence downgraded twice

3 Downgraded once due to imprecision

4 Only two (Santos 2009; Santos 2012) of three studies were suitable to pool to produce the following effect estimate: $0.48 \%(95 \% \mathrm{Cl}-0.54 \%$ to $1.51 \%)$ 


\section{B A C K G R O U N D}

\section{Description of the condition}

Diabetes mellitus (DM) is a chronic disease of the metabolism that is caused by the body's failure either to produce the hormone insulin or to effectively use its production of insulin. Insulin is a hormone produced by the pancreas that enables the body to direct glucose from the bloodstream to cells for energy. Without this vital hormone, glucose accumulates in the bloodstream and can result in disabling and life-threatening complications.

In 2014, the global prevalence of DM was estimated to be $8.3 \%$ among adults over 18 years old (WHO 2015). Estimates produced by the International Diabetes Federation suggest that 387 million people worldwide were affected in 2014, a number that is expected to grow to 592 million by 2035 (International Diabetes Federation 2013). In 2011, under the leadership of the World Health Organization, governments agreed a global action plan for the prevention and control of non-communicable diseases, with a target of reducing global premature deaths in the 30- to 70year-old age group by $25 \%$ by 2025 as part of its overall strategy (WHO 2013; WHO 2014).

Glycaemic control is a key issue in the care of people with DM. Prolonged hyperglycaemia is associated with complications such as retinopathy, peripheral neuropathy, macrovascular disease (coronary heart and cerebrovascular disease), foot disease (arising from a combination of vascular and neuropathic disease) and renal failure. The United Kingdom Prospective Diabetes Study (UKPDS) and the Diabetes Control and Complications trial in the USA have demonstrated that intensive treatment of hyperglycaemia can reduce the risk of long-term complications (DCCT 1993; Stratton 2000; UKPDS 1998). Each 1\% reduction in the haemoglobin A1c $(\mathrm{HbA} 1 \mathrm{c})$ in the UKPDS was associated with a relative risk reduction of $21 \%$ for any diabetes-related endpoint, $21 \%$ for diabetes-related deaths, $14 \%$ for myocardial infarction and $37 \%$ for microvascular complications (Stratton 2000). As part of this process, blood glucose levels may be monitored daily by the patient but also by regular haematological tests in a clinical laboratory. The HbA1c level is commonly measured to assess blood glucose levels over a period of approximately 6 to 8 weeks preceding the test and is recognised as a good indicator of glycaemic control, particularly as higher $\mathrm{HbAlc}$ levels are associated with an increased risk of diabetes-related complications (Bunn 1981).

Poorly controlled diabetes is also a well-recognised risk factor for developing periodontal disease (Papapanou 1996; Preshaw 2012; Seppälä 1993). There is epidemiological evidence that people with both type 1 diabetes mellitus (T1DM) and type 2 (T2DM) experience more periodontal disease, and periodontal disease of greater severity, than the general population (Firatli 1997; Sandberg 2000).

Periodontitis is defined as inflammation and destruction of the underlying supporting tissues of the teeth (the periodontium). In susceptible patients whose oral hygiene is suboptimal, a microbial biofilm (bacteria and extracellular substances) can form around the gum margin and result in inflammation and destruction of the periodontium. This complex, chronic disease requires lifelong control of the causative factors (Kornman 2014). Reduced periodontal support can lead to mobility (or drifting) of teeth, and ultimately tooth loss; this in turn may require additional treatment to restore lost function and appearance. Chronic inflammation of the periodontium may also lead to systemic inflammation more distantly.

The condition is categorised as aggressive or chronic (Armitage 1999). There is no subclass specific to DM, as it is recognised that diabetes may modify all forms of periodontal disease (Milward 2003). Disease severity is graded by measurement of clinical attachment levels (clinical attachment loss, pocket depth, or both if available). It has been estimated that the total surface area of inflamed and ulcerated epithelium of the periodontal tissues in an individual with periodontitis is at least equivalent to the surface area of the palm of the hand (Page 1998).

Observational studies have demonstrated that associations exist between socioeconomic status (SES: broadly includes ethnicity, income, social class, and education) variables and periodontal disease progression (low education and low income: Buchwald 2013), and SES (low income) and DM prevalence (Rabi 2006). Consequently, SES may confound observational studies of the association between DM and periodontal disease; however, adequate randomisation in trials of periodontal treatment will avoid such confounding.

In previous years, evidence has been published suggesting a bidirectional relationship between glycaemic levels and periodontal disease (Grossi 1998; Stewart 2001; Taylor 2001). In other words, the chronic inflammation and infection that results from periodontal disease could also have an adverse effect on glycaemic control in people with diabetes, which, in turn, could lead to worsening gum disease. Authoritative studies on DM such as DCCT 1993; Stratton 2000 and UKPDS 1998 did not collect data on periodontal disease or oral health in general.

\section{Description of the intervention}

Periodontal treatment includes a number of components of care. In many cases, oral hygiene instruction will be used to educate and motivate people to control for themselves the accumulation of causative factors, dental plaque, and bacterial biofilm. In addition, mechanical debridement (different forms of scaling, using conventional hand- or powered-instruments or both) by a dentist or hygienist is often required to remove both plaque and plaque deposits that have mineralised and hardened (calculus). These deposits can form both above and below the gingival margin.

With more advanced forms of disease, surgery can be needed to lift the gingival tissues away from the tooth, facilitating access to clean away the deposits when located below the gum line. Some of these measures require several visits. Antimicrobials (inclusive of antiseptics, antibacterials and antibiotics) have also been used as adjuncts to scaling, although without evidence of a clear benefit (Bonito 2004).

\section{How the intervention might work}

Any improvement in glycaemic control resulting from regular and appropriate periodontal treatment has the potential to make a significant impact on the development of diabetic complications and on quality of life for people with diabetes. We would have included evidence of cost implications of treatment if these had been available from the studies. This review aims to investigate the influence of periodontal treatment upon glycaemic control. 


\section{Why it is important to do this review}

Cochrane Oral Health undertook an extensive prioritisation exercise in 2014 to identify a core portfolio of titles that were the most clinically important ones to maintain on the Cochrane Library (Worthington 2015). This review was identified as a priority title by the periodontal expert panel (Cochrane OHG priority review portfolio). This is an update of the Cochrane review first published in 2010 (Simpson 2010).

The cost to governmental health budgets of managing people with diabetes is substantial. The global cost of diabetes care has been estimated to be USD 612 billion (International Diabetes Federation 2013). The spending on diabetes-related disease has been found to be positively associated with the gross domestic product of countries (Seuring 2015). The economic burden on the UK was estimated to be approximately GBP 9.8 billion in $2010 / 11$ or $10 \%$ of the National Health Service (NHS) budget, with GBP 8.8billion of this amount relating to treatment for people with T2DM, and a further projected rise to $17 \%$ of health service resources by 2035/2036 (Hex 2012).

If there is a direct benefit of periodontal therapy on glycaemic control, the implications may be profound.

- Periodontal disease, which is prevalent in most populations, could be an additional confounder in studies of the effect of glycaemic control.

- Readily available treatments by dentists and auxiliary workers could have a marked effect in improving glycaemic control among people with diabetes.

\section{OB JECTIVES}

The objective is to investigate the effect of periodontal therapy on glycaemic control in people with diabetes mellitus.

\section{METHODS}

\section{Criteria for considering studies for this review}

\section{Types of studies}

Randomised controlled trials (RCTs) were eligible for inclusion.

We excluded trials if the participants were followed up for less than 90 days after completion of the treatment course. We excluded split-mouth and cross-over studies, due to the anticipated influence of carry-over effects from treatment.

\section{Types of participants}

We included studies of people with diabetes mellitus and periodontal disease who were at least 16 years of age. We analysed participants as having a diagnosis of type 1 diabetes mellitus (T1DM) if, at the beginning of the study, the individual's disease classification was juvenile-onset diabetes, type I or insulindependent DM (IDDM). If an individual was described as having adult-onset, type II or non-insulin dependent DM (NIDDM), we analysed the data as for that of a participant with type 2 diabetes mellitus (T2DM).

For periodontitis, we accepted trialist statements that participants were selected on the basis of a diagnosis of chronic or adult periodontitis and we would have investigated the effect of adequacy of diagnosis on the outcomes if sufficient data had been available.

We included studies regardless of the general medical health of the participants. No restriction was placed on setting - primary care, hospital or community were all considered. We would have excluded studies if more than $10 \%$ of the study sample had been diagnosed with gestational diabetes (diabetes associated with pregnancy). We did not include studies where participants were described as having metabolic syndrome.

\section{Types of interventions}

Periodontal treatments (any professionally-delivered intervention designed to reduce periodontal disease) should have included one or more of the following:

- mechanical debridement (also called non-surgical periodontal treatment) - scaling, root planing, subgingival curettage;

- surgical periodontal treatment - flap surgery or gingivectomy;

- antimicrobial therapy (encompassing antibacterials and antibiotics), either locally applied (including mouthrinses, gels or dentifrices) or systemically administered;

- other drug therapy with a possible benefit of improving the periodontal condition of the participant;

- other novel interventions to manage periodontal disease.

Periodontal treatments may also have included either of the following adjuncts as part of 'usual' care:

- oral hygiene instruction;

- education or support sessions to improve self help or self awareness of oral hygiene.

Interventions were compared with no treatment, 'usual care' (for example, supragingival prophylaxis, standalone oral hygiene instruction) or placebo.

Trials that made direct comparisons between different types of periodontal treatment were also included (eg adjunctive drug therapies (including multiple versus single), rapid versus standard delivery methods etc) to identify whether providing enhanced forms of periodontal treatment is of additional benefit for improving glycaemic control.

RCTs comparing surgical against non-surgical periodontal treatment would also have been included within this review if any had been found.

\section{Types of outcome measures}

A number of different blood indices have been identified as indicators of blood glucose levels and therefore, possible prognostic markers. The glycated (glycosylated) haemoglobin assay (HbA1c) gained widespread acceptance during the 1980s as the laboratory test of choice and is still widely used. HbA1c has been measured using a number of differing methods with several internationally adopted standards. These include the Diabetes Control and Complications Trial (DCCT) or the International Federation of Clinical Chemistry (IFCC) standard tests (their respective standardised values were implemented globally after achieving consensus in 2007 before being refined further in 2009 (Hanas 2010)). The latter consistently gives lower values (nondiabetic reference range is about $3 \%$ to $5 \%$ IFCC and $4 \%$ to $6 \%$ 
DCCT, with good control in diabetic groups as $5 \%$ IFCC and $7 \%$ DCCT. Treatment alteration becomes a requirement with values $>6 \%$ IFCC and $>8 \%$ DCCT (Florkowski 2003). We noted methods and reference ranges where given and would have subjected these to sensitivity analysis had the information been available.

Measures of glycaemic control may, therefore, not be comparable between studies, but the focus of this review was internal comparisons. Some studies measured blood glucose levels (such as plasma glucose fasting levels); however, we did not feel that it was appropriate to use this as an outcome measure. Whilst blood glucose is useful for management on a daily basis (particularly in T1DM), it can be very variable and heavily influenced by many factors like diet, exercise etc. HbAlc gives a better measure of long-term glycaemic control and is shown to be more strongly associated with complications of diabetes than blood glucose (Goldstein 2004).

\section{Primary outcomes}

- The absolute percentage change from baseline in $\mathrm{HbA1C}$ - from pre-treatment for periodontal condition to at least 90 days posttreatment.

The minimum of 3-month follow-up duration, for including studies in this review, is clinically justified due to human red blood cells ordinarily having a lifespan of between 8 to 12 weeks (Franco 2012).

We excluded trials that did not measure $\mathrm{HbAlc}$ as an outcome (ie where $\mathrm{HbA1c}$ is not reported in the trial report or these data are not available from the trial authors).

\section{Secondary outcomes}

- Changes in periodontal attachment level.

- Gingival indices (inflammation or bleeding or both).

- Plaque indices.

- Any adverse effects of treatment.

- Quality of life indicators (eg hospital anxiety and depression scale (HADS), health-related quality of life (HRQoL)).

- Cost implications.

- Diabetic complications.

\section{Search methods for identification of studies}

\section{Electronic searches}

For the identification of studies for this review, we developed detailed search strategies for each database searched. These were based on the search strategy developed for MEDLINE but revised appropriately for each database to take account of differences in controlled vocabulary and syntax rules.

The MEDLINE search strategy was linked with the Cochrane Highly Sensitive Search Strategy (CHSSS) for identifying randomised trials (RCTs) in MEDLINE: sensitivity maximising version (2008 revision) as referenced in Chapter 6.4.11.1 and detailed in box 6.4.c of the Cochrane Handbook for Systematic Reviews of Interventions Version 5.1.0 (updated March 2011) (Higgins 2011). The searches of EMBASE and CINAHL were linked to the Cochrane Oral Health Group filters for identifying RCTs, and the search of LILACS was linked to the Brazilian Cochrane Center filter.

We searched the following databases:
- the Cochrane Oral Health Group Trials Register (to 31 December 2014);

- Cochrane Central Register of Controlled Trials (CENTRAL) (Cochrane Library 2014, Issue 11);

- MEDLINE via OVID (1946 to 31 December 2014);

- EMBASE via OVID (1980 to 31 December 2014);

- CINAHL via EBSCO (1937 to 31 December 2014);

- LILACS via BIREME Virtual Health Library (1982 to 31 December 2014);

- ISI Web of Knowledge (conference proceedings) (1990 to 31 December 2014);

- ZETOC (conference proceedings) (1993 to 31 December 2014).

See Appendix 1 for details of all search strategies.

\section{Searching other resources}

We searched the following databases for ongoing trials:

- US National Institutes of Health Trials Registry (http:// clinicaltrials.gov) (to 31 December 2014);

- WHO Clinical Trials Registry Platform (http://apps.who.int/ trialsearch/default.aspx) (to 31 December 2014).

No restrictions were placed on the language of publication when searching the electronic databases, or reviewing reference lists in identified studies or reviews. We attempted to contact known authorities, as identified by the Cochrane Oral Health Group, in the following languages for information about publications, which might contain relevant trials: Japanese, Chinese, German, French and Spanish. In addition to this, any papers we identified by any of the database searches that were in a language other than English were translated and considered for inclusion.

We contacted authors of relevant studies for clarification regarding their own studies and for information regarding other studies of which they are aware.

\section{Handsearching}

The review authors handsearched the following journals:

- Annals of Periodontology (1996 to 2003);

- Periodontology 2000 (1993 to 2003).

We did not handsearch any medical or specialist journals relating to diabetes.

We scrutinised known papers previously published on the topic for potentially relevant references.

\section{Data collection and analysis}

\section{Selection of studies}

Two review authors screened all titles (and abstracts if available) in duplicate. We rejected only clearly irrelevant records at this stage. We retrieved and examined the full text of potentially relevant studies. Four teams of two review authors independently extracted data in duplicate. Where authors disagreed on studies for inclusion, another review author acted as arbiter. The review authors were not blinded to the authors of the studies, as this has been shown to be of dubious value, but adds a significant amount of time to completion of the process (Berlin 1997). 
We maintained a detailed log of study eligibility and reasons for exclusion.

\section{Data extraction and management}

We collected data on a pre-determined and piloted form. The following characteristics of each study were recorded on the data extraction form.

- General characteristics - year of study, language of original publication, country of origin, funding.

- Trial design - sample size, method of allocation, blinding and comparative group characteristics.

- Population studied - ethnic groups, setting, social class, whether T1DM or T2DM (or both), duration of diabetes, duration of diabetic control $^{\star}$, other stated medical conditions, type of periodontal disease (gingivitis only, chronic/adult periodontitis, aggressive/early-onset periodontitis), smoking habits, alcohol consumption, drug therapy.

- Nature of the intervention - oral hygiene, self administered measures, type of periodontal therapy and antimicrobial/ antiseptics employed, compliance.

- Primary outcomes - HbAlc at baseline, during therapy and posttherapy (and where available: test method; reference values; corresponding DCCT/IFCC standards).

- Secondary outcomes - changes in clinical attachment level (CAL), probing pocket depth (PPD), bleeding on probing $(\mathrm{BOP})$, gingival index $(\mathrm{GI})$ and plaque index $(\mathrm{PI})$. Also diabetic complications and changes in antidiabetic therapy.

* It would be of interest to be able to narratively report the proportion of the population whose diabetes control was considered to be well controlled over a longer period of time, to provide context to the findings of this review, which is based on singular HbAlc measures (at follow-up time points).

Two review authors independently extracted numerical data into data tables and Review Manager (RevMan) software (RevMan 2014). A third review author verified the data inputted into RevMan.

\section{Diagnostic assessment}

This was assessed as.

- Diabetes mellitus diagnosis: criteria for diagnosis clearly defined (and consistent with relevant classification in use during study conduct period): adequate, inadequate, unclear.

- Adequate: prior T1DM/T2DM diagnosis (determined by either description of clinical diagnosis in publication or information from study authors).

- Inadequate: participants self report/identify as diabetic without clinical confirmation.

- Unclear: no information provided about T1DM/T2DM diagnosis.

- Periodontal disease diagnosis: criteria for diagnosis clearly defined: adequate, inadequate, unclear.

- Adequate: at least two sites with probing depth of $\geq 5 \mathrm{~mm}$ with $\geq 2 \mathrm{~mm}$ loss of clinical attachment and/or alveolar bone loss of more than $4 \mathrm{~mm}$.

- Inadequate: less than two sites with probing depth of $\geq 5 \mathrm{~mm}$ with $\geq 2 \mathrm{~mm}$ loss of clinical attachment and/or alveolar bone loss of more than $4 \mathrm{~mm}$.
- Unclear: no criteria given.

The diagnostic methods are summarised in Additional Table 1.

\section{Assessment of risk of bias in included studies}

We assessed studies against the following risk of bias criteria, in accordance with the Cochrane Handbook for Systematic Reviews of Interventions 5.1.0 (Higgins 2011):

- random sequence generation;

- allocation concealment;

- blinding of participants;

- blinding of clinical operator;

- incomplete outcome data;

- selective outcome reporting;

- other biases.

We assessed each domain as being at low, high or unclear risk of bias. 'Unclear' indicates either lack of information or uncertainty over the potential for bias.

\section{Measures of treatment effect}

For continuous outcomes (eg HbAlc, clinical outcomes) where studies used the same scale, we used the mean values and standard deviations (SDs) reported in the studies in order to express the estimate of effect as mean difference (MD) with 95\% confidence interval $(\mathrm{Cl})$. If different scales had been used, we would have considered expressing the treatment effect as standardised mean difference (SMD) with $95 \% \mathrm{Cl}$.

If there had been any dichotomous outcomes we would have expressed the estimate of effect as a risk ratio (RR) with $95 \% \mathrm{Cl}$.

\section{Dealing with missing data}

We attempted to contact trial authors to retrieve missing data when they were not available from the trial report, or to clarify areas where data or trial design and conduct were unclear. If we received no response, we excluded the data from meta-analyses until clarified by the study authors. Where standard deviations were missing we obtained these from a study's confidence intervals, $\mathrm{P}$ values or t-values where available.

\section{Assessment of heterogeneity}

We assessed statistical heterogeneity by calculation of the ' $Q$ ' statistic with $P$ value set at $P<0.10$. This was quantified by the calculation of the $\mathrm{I}^{2}$ statistic for heterogeneity.

\section{Assessment of reporting biases}

Where there were sufficient studies (more than 10 per comparison), we assessed publication bias by graphical methods (funnel plots, which indicate potential presence of reporting biases by testing for asymmetry) and via the Begg and Mazumdar adjusted rank correlation test (Begg 1994) and the Egger et al regression asymmetry test (Egger 1997).

\section{Data synthesis}

We conducted meta-analysis where studies were judged to be sufficiently similar. We used random-effects meta-analyses to combine quantitative data, where there were at least four 
studies. All the data analysed were continuous. We expressed pooled outcomes as mean differences with their associated $95 \%$ confidence intervals. Where single studies provided data for two subgroups with a common control group, which were pooled, half of the study's control group was used in each subgroup to avoid double-counting participants.

\section{Subgroup analysis and investigation of heterogeneity}

If there were sufficient studies, we would have used sensitivity analyses and meta-analysis regression (using STATA software) to explore, quantify, and control for sources of heterogeneity between studies for the following quality criteria and prognostic factors:

- study quality;

- periodontal disease severity (initial probing depth);

- T1DM and T2DM;

- DM control - through categorisation of patients into good, fair and poor (mean $\mathrm{HbA} 1 \mathrm{c} 7 \%$, between $7 \%$ and $8.5 \%$ or $>8.5 \%$ on the DCCT or equivalent scale);

- DM duration (since diagnosis);

- age;

- sex;

- smoking habits;

- alcohol consumption;

- general health status;

- presence of other medical conditions;

- plaque control;

- socioeconomic status;

- drug therapy.

\section{Sensitivity analysis}

We performed sensitivity analyses (where there were sufficient studies for each outcome) by excluding studies at high and unclear risk of bias in order to ensure our conclusions were robust.

\section{Summarising findings and assessing the quality of the evidence}

We developed 'Summary of findings' tables for the primary outcomes of this review using GRADEpro software (GRADEpro). We assessed the quality of the body of evidence with reference to the overall risk of bias of the included studies, the directness of the evidence, the inconsistency of the results, the precision of the estimates, the risk of publication bias, and the magnitude of the effect. The quality of the body of evidence for each of the primary outcomes was categorised as high, moderate, low or very low (GRADEpro).

\section{RE S U L T S}

\section{Description of studies}

\section{Results of the search}

The literature search for this review update identified 688 records after the duplicates were removed. These 688 records were screened independently and in duplicate. After screening, we retained 74 records for further assessment and categorised 614 records as not relevant.

We tried to obtain full texts of 74 records, but only found 62 full-text articles as 12 studies were still ongoing. We also found two studies (Calbacho 2004; Singh 2008) in the bibliographies of reviews (Darré 2008; Engebretson 2013a; Sgolastra 2013).

Following our assessment of the 64 full-text articles (including Calbacho 2004 and Singh 2008) from this updated search, we excluded a total of 12 studies (12 articles) with reasons provided (Characteristics of excluded studies), and we categorised five studies (seven articles) as awaiting classification at the next update of this review once required information has been identified (Characteristics of studies awaiting classification).

We included 35 studies (a total of 45 articles, including seven already included studies from the previous version of the review), of which 34 studies (all except Madden 2008) provided useable data. Figure 1 shows the study selection process. 
Figure 1. Review update: study flow diagram

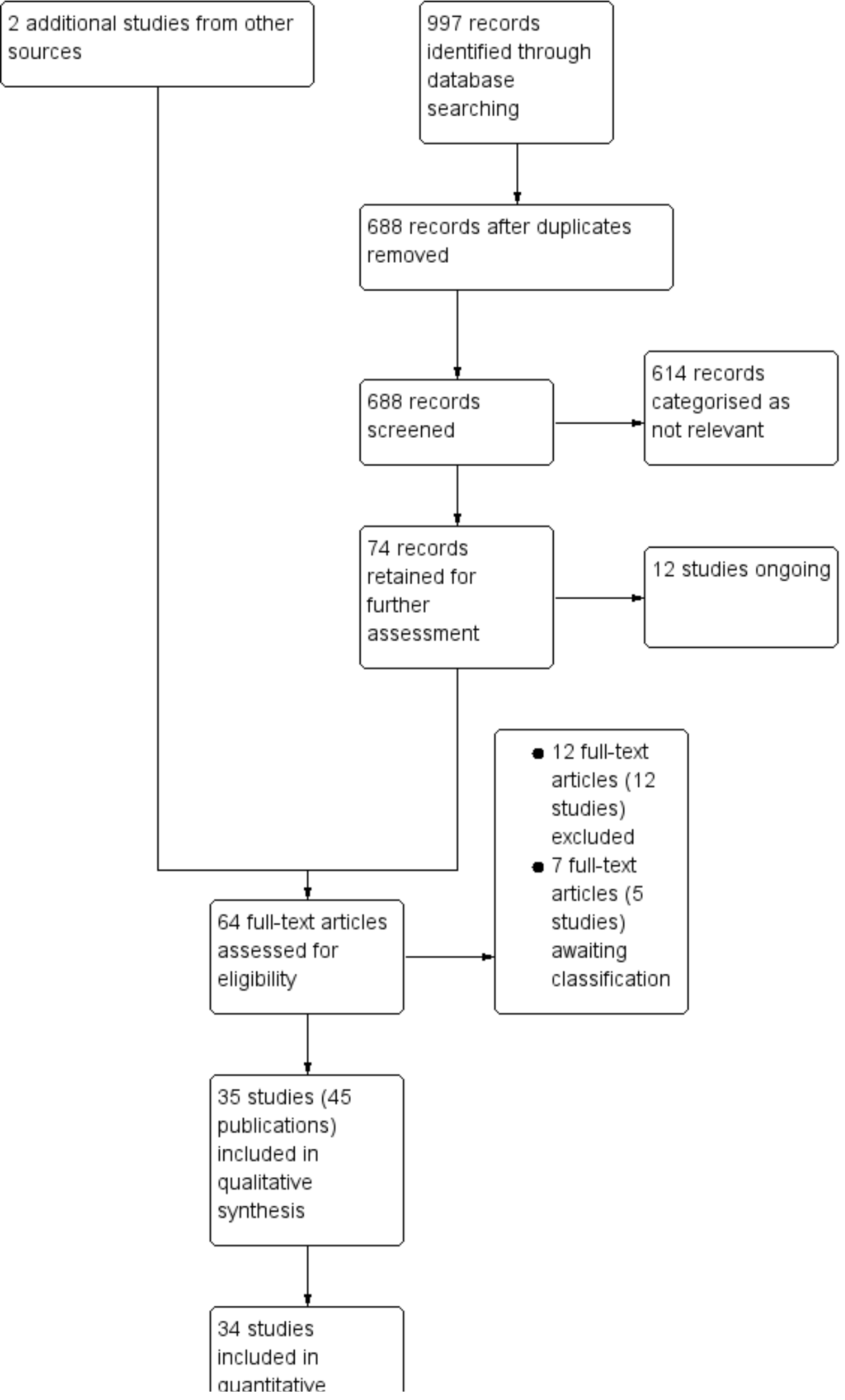


Figure 1. (Continued)

included in
quantitative
synthesis
(meta-analysis)

\section{Included studies}

See Characteristics of included studies table.

Thirty-five studies (involving a total of 2565 participants) met our criteria for inclusion, and all studies except one (Madden 2008) reported usable results for at least one of our two comparisons.

- Does periodontal therapy improve glycaemic control in people with diabetes mellitus?

- Does one periodontal therapy have a greater effect than another on improving glycaemic control?

\section{Characteristics of studies}

\section{Setting}

The included studies were conducted in the following countries:

- Brazil (seven trials, 20\%: Macedo 2014; Miranda 2014; O'Connell 2008; Rodrigues 2003; Santos 2009; Santos 2012; Santos 2013);

- USA (seven trials, 20\%: Engebretson 2011; Engebretson 2013; Gay 2014; Grossi 1997; Jones 2007; Madden 2008; NCT00801164);

- China (five trials, 14\%: Chen 2012; Li 2011; Sun 2011; Yun 2007; Zhang 2013);

- India (three trials, 9\%: Kothiwale 2013; Pradeep 2013; Singh 2008);

- Greece (two trials, 6\%: Koromantzos 2011; Tsalikis 2014);

- Iran (two trials, 6\%: Haerian Ardakani 2014; Moeintaghavi 2012); and

- nine countries (totaling 26\%) conducted one included study (3\%) each (Chile: Calbacho 2004; Japan: Katagiri 2009; Malaysia: Raman 2014; Mexico: Rocha 2001; Poland: Gilowski 2012; Saudi Arabia: Al-Zahrani 2009; Slovenia: Skaleric 2004; Spain: Llambés 2008; Turkey: Kiran 2005).

The majority of trials $(n=24 ; 69 \%)$ were conducted in a hospital setting; two studies (6\%) were conducted in a primary care setting (Calbacho 2004; Jones 2007); two trials (6\%) were conducted in a community setting (Engebretson 2013; Li 2011), and seven trials (20\%) did not report the type of setting (Chen 2012; Gilowski 2012; Grossi 1997; Macedo 2014; Madden 2008; O'Connell 2008; Rocha 2001).

Twenty-five trials ( $71 \%$ ) were conducted from a single centre; three trials (9\%) did not report how many centres they conducted their trials from (Calbacho 2004; Gilowski 2012; Madden 2008), and seven trials (20\%) were multicentred (Engebretson 2013; Jones 2007; Katagiri 2009; Li 2011; Raman 2014; Tsalikis 2014; Yun 2007).

\section{Design}

All studies used a parallel randomised controlled trial (RCT) design. Twenty-nine studies (83\%) had a two-arm design; five studies (14\%) had a three-arm design (Al-Zahrani 2009; Chen 2012; Engebretson
2011; Li 2011; Singh 2008), and one study (3\%) contained five arms (Grossi 1997).

\section{Follow-up}

Length of follow-up varied amongst the 35 included studies, ranging from 3 to 12 months duration.

- 14 studies (40\%) followed up their participants for a period of 3 months (Al-Zahrani 2009; Engebretson 2011; Gilowski 2012; Haerian Ardakani 2014; Kiran 2005; Kothiwale 2013; Llambés 2008; Macedo 2014; Moeintaghavi 2012; O'Connell 2008; Raman 2014; Rodrigues 2003; Singh 2008; Sun 2011).

- Four trials (11\%) had a follow-up period of 4 months (Calbacho 2004; Gay 2014; Jones 2007; Yun 2007). It was agreed by the review authors that there was no clinically significant difference between 3 and 4 months, and therefore these reported timepoints have been pooled within the meta-analyses in this review as '3-4 months.'

- 12 trials (34\%) followed up their participants for 6 months (Chen 2012; Engebretson 2013; Grossi 1997; Katagiri 2009; Koromantzos 2011; Li 2011; NCT00801164; Rocha 2001; Santos 2009; Skaleric 2004; Tsalikis 2014; Zhang 2013).

- One study (3\%) had a follow-up period of 8 months (Madden 2008).

- One study (3\%) had a follow-up period of 9 months (Pradeep 2013).

- Three studies (9\%) followed up participants for 12 months (Miranda 2014; Santos 2012; Santos 2013).

\section{Funding}

Studies were funded by a variety of sources.

- Six of the included studies (17\%) were funded by São Paulo State Research Foundation (Macedo 2014; Miranda 2014; O'Connell 2008; Rodrigues 2003; Santos 2012; Santos 2013).

- Six studies (17\%) were funded by other government sponsors (Chen 2012; Katagiri 2009; Li 2011; Rocha 2001; Sun 2011; Zhang 2013).

- Five studies (14\%) were funded by universities (Al-Zahrani 2009; Gilowski 2012; Madden 2008; Moeintaghavi 2012; Raman 2014).

- Four studies $(11 \%)$ were funded by research institutes (Engebretson 2013; Gay 2014; Grossi 1997; Koromantzos 2011).

- Two studies $(6 \%)$ were jointly funded by universities and research institutes (Engebretson 2011; Jones 2007).

- Two studies (6\%) were self funded (Llambés 2008; Singh 2008 (stating no source of support)).

- One study (3\%) was fully funded by an industry award (Tsalikis 2014).

- One study (3\%) was partially funded by industry (NCT00801164). 
- Eight studies (23\%) did not report their source of funding (Calbacho 2004; Haerian Ardakani 2014; Kiran 2005; Kothiwale 2013; Pradeep 2013; Santos 2009; Skaleric 2004; Yun 2007).

\section{Conflicts of interest}

In 15 of the included studies (43\%), all authors declared no conflict of interests (Al-Zahrani 2009; Chen 2012; Engebretson 2011; Gay 2014; Katagiri 2009; Koromantzos 2011; Miranda 2014; O'Connell 2008; Pradeep 2013; Raman 2014; Santos 2009; Santos 2013; Singh 2008; Sun 2011; Tsalikis 2014); in one study (3\%), conflict of interest declarations were available for all authors except the lead author (Engebretson 2013); and two studies (5\%) have yet to be published, therefore, no declaration of conflict of interests currently exists to report (Kothiwale 2013; NCT00801164). Declarations regarding conflict of interests were not reported in the remaining $17(49 \%)$ included studies.

\section{Study primary outcomes and sample size calculations}

Of the 35 included studies, only 11 (31\%) reported HbA1c to be their studies' primary outcome. Of these 11 studies:

- five studies (14\%) were sufficiently powered to detect a statistically significant difference (Engebretson 2013; Gay 2014; Koromantzos 2011;Raman 2014; Zhang 2013);

- two studies (6\%) were underpowered, due to randomising fewer participants than their own sample size calculation required (Chen 2012; Jones 2007);

- two studies (6\%) did not report their sample size calculation (Engebretson 2011; Kothiwale 2013); and

- two studies (6\%) indicated a sample size calculation had been undertaken, but did not report details and may not have been calculated a priori (Llambés 2008; Madden 2008).

Of the remaining included studies:

- five studies (14\%) reported probing pocket depth (PPD) to be their studies' primary outcome (sufficiently powered: Al-Zahran 2009; Gilowski 2012; Macedo 2014; Miranda 2014. Calculation not reported: Li 2011);

- four studies (12\%) reported clinical attachment level (CAL) to be their studies' primary outcome (sufficiently powered: Santos 2009; Santos 2012; Santos 2013; Tsalikis 2014);

- one study (3\%) reported bone defect fill to be their study's primary outcome (sufficiently powered: Pradeep 2013); and

- 14 studies (40\%) did not report their primary outcome. Of these, 12 studies (34\%) did not report their sample size calculation (Calbacho 2004; Grossi 1997; Haerian Ardakani 2014; Katagiri 2009; Kiran 2005; O'Connell 2008; Rocha 2001; Rodrigues 2003; Singh 2008; Skaleric 2004; Sun 2011; Yun 2007), one study (3\%) has yet to be published but states a required sample size in its trial register record (NCT00801164), and the remaining study (3\%) broadly based their sample size upon figures used in two previous studies (Moeintaghavi 2012 using Kiran 2005 and Rodrigues 2003).

\section{Analysis method}

Of the 35 included studies, 15 reported (43\%) they had analysed their participant data using intention-to-treat (ITT) (Calbacho 2004; Engebretson 2011; Engebretson 2013; Haerian Ardakani 2014; Katagiri 2009; Kiran 2005; Koromantzos 2011; Macedo 2014; Miranda 2014; Moeintaghavi 2012; Santos 2009; Santos 2012;
Santos 2013; Skaleric 2004; Yun 2007); 12 studies (34\%) analysed their participant data as per protocol (Chen 2012; Gay 2014; Grossi 1997; Jones 2007; Kothiwale 2013; Llambés 2008; NCT00801164; O'Connell 2008; Raman 2014; Sun 2011; Tsalikis 2014; Zhang 2013); and the method of data analysis was not reported in eight studies (23\%) (Al-Zahrani 2009; Gilowski 2012; Li 2011; Madden 2008; Pradeep 2013; Rocha 2001; Rodrigues 2003; Singh 2008).

\section{Characteristics of participants}

The largest study (Engebretson 2013) included 514 participants, accounting for $20 \%$ of the total 2565 participants included in this review. Each of the other 34 studies included in the review contained less than 200 participants each.

Thirty-two studies (91\%) included only T2DM participants; one study (3\%) assumed participants to all be T2DM without confirmed diagnosis (Jones 2007); and two studies (6\%) only included T1DM participants (Llambés 2008; Skaleric 2004). Only 16 included studies (47\%) reported $\mathrm{HbAlc}$ criteria for participants at study entry. There was substantial variation between these trials in both the level and range of $\mathrm{HbAlc}$ of participants at baseline, with consequent variation in the potential for improvement in glycaemic control as a result of the intervention. Diabetic control of participants at baseline is summarised in Additional Table 2. The breadth of included studies' diabetic control $\mathrm{HbAlc}$ thresholds for participant inclusion varied greatly amongst included trials and is summarised in Additional Table 2.

Included trials spanned a broad range of age groups from 18 to 80 years. Eight studies (22\%) did not report an age range for inclusion in their trials (Engebretson 2011; Haerian Ardakani 2014; Jones 2007 (reported only as "military veterans"); Li 2011; Moeintaghavi 2012; O'Connell 2008; Rodrigues 2003; Yun 2007).

Twenty-five studies $(71 \%)$ contained a reasonably even balance of males and females; five studies (14\%) contained a two-thirds balance of sexes (in favour of males: Kothiwale 2013; Raman 2014; in favour of females: Grossi 1997; Kothiwale 2013; Santos 2013); one study (3\%) almost exclusively contained male participants (Jones 2007); and proportion of male to female participants was not reported in four studies (11\%) (Haerian Ardakani 2014; NCT00801164; Rodrigues 2003; Singh 2008).

Use of antidiabetic therapy varied greatly amongst included trials and is summarised in Additional Table 3. There was also variation between trials where participants' antidiabetic therapy was changed during the trial conduct period:

- in 11 studies (31\%) there were no changes to prescribed antidiabetic therapy (Al-Zahrani 2009; Calbacho 2004; Engebretson 2011; Katagiri 2009; Kiran 2005; Kothiwale 2013; Moeintaghavi 2012; Santos 2009; Santos 2012; Santos 2013; Singh 2008);

- in 10 studies (29\%) some participants' prescribed antidiabetic therapy was changed (Engebretson 2013; Gay 2014; Grossi 1997; Jones 2007; Koromantzos 2011; Llambés 2008; Madden 2008; Raman 2014; Rodrigues 2003; Skaleric 2004); and

- for 14 studies (40\%), it is unknown whether any changes were made to participants' prescribed antidiabetic therapy (Chen 2012; Gilowski 2012; Haerian Ardakani 2014; Li 2011; Macedo 2014; Miranda 2014; NCT00801164; O'Connell 2008; Pradeep 
2013; Rocha 2001; Sun 2011; Tsalikis 2014; Yun 2007; Zhang 2013).

Additional Table 4 describes the changes made to antidiabetic therapy, as reported by each included study.

Included studies made a variety of participant exclusions. Most frequently reported exclusion criteria were antibiotic use (largely in prior 3 months, where reported) in 28 trials (80\%), pregnancy (including breastfeeding/lactation) in 25 trials (71\%) and tobacco use in 17 trials (49\%).

Other notable exclusions were:

- periodontal treatment (largely in prior 3 months, where reported) in 10 studies (29\%);

- immunosuppressed or compromised patients in 10 studies $(29 \%)$;

- systemic disease in 10 studies (29\%);

- non-steroidal anti-inflammatory drugs (NSAID) use (largely in prior 3 months, where reported) in nine studies (26\%);

- diabetic complications in eight studies $(23 \%)$;

- orthodontic appliance use in six studies (17\%);

- renal/liver dysfunction in five studies (14\%);

- alcohol/drug abuse in four studies (11\%);

- cardiovascular disease/events in four studies (11\%).

Rheumatoid arthritis and anticoagulant use were each exclusion criteria in two studies (6\%), and stroke and insulin use were each excluded from single trials (3\%). Grossi 1997 did not report any exclusion criteria, and unpublished author content relating to exclusions for Calbacho 2004 was unintelligible (request for clarification was sought but no response received)

\section{Characteristics of assessments}

All included studies used adequate criteria for diagnosis of diabetes mellitus (Additional Table 1). Few trials specifically indicated whether they had used IFCC or DCCT standard test scales; however, given the recorded $\mathrm{HbAlc}$ values at baseline, it is likely most studies used the DCCT standard.

Diagnostic criteria for periodontal disease (also Additional Table 1 ) was assessed as adequate in 11 studies (31\%) (Engebretson 2013; Gay 2014; Koromantzos 2011; Llambés 2008; Macedo 2014; Moeintaghavi 2012; Raman 2014; Santos 2009; Sun 2011; Tsalikis 2014; Zhang 2013), and unclear in four studies (11\%) (Calbacho 2004; Grossi 1997; Kiran 2005; Li 2011) due to either not reporting this detail or offering no defined criteria. Diagnosis of periodontal disease was assessed as inadequate in 20 studies (57\%) for a variety of reasons:

- insufficient indication of criteria in two trials (6\%) (Kothiwale 2013; Madden 2008);

- less than two sites required in three trials (9\%) (Miranda 2014; O'Connell 2008; Rodrigues 2003);

- partial criteria use in nine trials (26\%) (PPD only, no CAL/bone loss: Gilowski 2012; Haerian Ardakani 2014; Jones 2007; Katagiri 2009; Skaleric 2004; Yun 2007. CAL only, no PPD/bone loss: AlZahrani 2009; Engebretson 2011; NCT00801164);

- no indication of number of sites in one trial (3\%: Pradeep 2013); and
- a lower threshold was used for PPD/CAL in five trials (14\%) (CAL $\geq 1 \mathrm{~mm}$ : Chen 2012; PPD >3 mm: Rocha 2001; PPD/CAL $\geq 4$ mm: Santos 2012; Santos 2013; Singh 2008).

\section{Characteristics of interventions and comparisons}

Interventions compared by the included studies are shown in Additional Table 5.

All included studies assessed the use of non-surgical periodontal therapy with usual care/no active treatment and/or compared different types of periodontal therapy (periodontal therapy delivered in both arms, with intervention groups receiving more intensive treatment delivery compared to that received by control groups. Combined interventions varied across studies, and are clearly detailed in Additional Table 5 within this review). No included studies compared surgical with non-surgical periodontal interventions.

Twenty-seven studies (77\%) included the use of oral hygiene instruction (OHI) (treatment arm only ( $n=7 ; 20 \%$ : Chen 2012; Kiran 2005; Kothiwale 2013; Singh 2008; Sun 2011; Yun 2007; Zhang 2013), control arm only ( $\mathrm{n}=1 ; 3 \%$ : Calbacho 2004), or both arms ( $\mathrm{n}=$ 19; 54\%: Al-Zahrani 2009; Engebretson 2013; Gay 2014; Gilowski 2012; Katagiri 2009; Koromantzos 2011; Llambés 2008; Macedo 2014; Madden 2008; Miranda 2014; O'Connell 2008; Pradeep 2013; Raman 2014; Rocha 2001; Rodrigues 2003; Santos 2009; Santos 2012; Santos 2013; Tsalikis 2014).

Included studies' interventions ranged greatly between single and multiple delivery sessions (repeated up to a maximum of seven sessions, for example, in Macedo 2014 and Rodrigues 2003), and great variability also existed in the repetition of intervention components within studies.

For comparison 1 , the only subgroup analyses we were able to undertake involved the use of adjunctive antimicrobials (without/ with). For comparison 2, we were able to combine studies in to seven subgroups for analysis at each time-point; however, we were only able to derive an effect estimate from pooled studies within the subgroup comparing scaling and root planing (SRP) plus antimicrobials with SRP, due to the other subgroups comprising of multiple head-to-head intervention comparisons which were unsuitable to be combined to estimate subgroup effects.

\section{Primary outcomes}

HbA1c: 33 studies (94\%) reported at 3-4 months (Al-Zahrani 2009; Calbacho 2004; Chen 2012; Engebretson 2011; Engebretson 2013; Gay 2014; Gilowski 2012; Grossi 1997; Haerian Ardakani 2014; Jones 2007; Katagiri 2009; Kiran 2005; Koromantzos 2011; Kothiwale 2013; Li 2011; Llambés 2008; Macedo 2014; Miranda 2014; Moeintaghavi 2012; NCT00801164; O'Connell 2008; Pradeep 2013; Raman 2014; Rodrigues 2003; Santos 2009; Santos 2012; Santos 2013; Singh 2008; Skaleric 2004; Sun 2011; Tsalikis 2014; Yun 2007; Zhang 2013), and 17 studies (49\%) reported at 6 months (Chen 2012; Engebretson 2013; Grossi 1997; Katagiri 2009; Koromantzos 2011; Li 2011; Madden 2008; Miranda 2014; NCT00801164; Pradeep 2013; Rocha 2001; Santos 2009; Santos 2012; Santos 2013; Skaleric 2004; Tsalikis 2014; Zhang 2013).

\section{Secondary outcomes}

- Bleeding on probing (BOP): 15 studies (43\%) reported at 3-4 months; eight studies (23\%) reported at 6 months. 
- Clinical attachment level (CAL): 20 studies (57\%) reported at 3-4 months; 14 studies (40\%) reported at 6 months.

- Gingival index (GI): nine studies (26\%) reported at 3-4 months; six studies (17\%) reported at 6 months.

- Plaque index (PI): 19 studies (54\%) reported at 3-4 months; nine studies (26\%) reported at 6 months.

- Probing pocket depth (PPD): 23 studies (66\%) reported at 3-4 months; 13 studies (37\%) reported at 6 months.

- Adverse effects: 15 studies (43\%) reported data relating to adverse events.

- No included studies reported data relating to quality of life, cost implications or diabetic complications.

\section{Excluded studies}

After examination of full-text papers, we excluded 12 studies as they failed to meet the criteria stated in the protocol.

The reasons for exclusion are detailed in the Characteristics of excluded studies tables; however, a synopsis of exclusion rationale follows:

- non-randomised design ( $n=3$ : Munenaga 2013; Promsudthi 2005 (patients may self select to control group in both studies); Taylor 2011 (error in MEDLINE reference, is actually a review));

- HbA1c not reported ( $n=2$ : Albrecht 1988; Llambés 2012);

- insufficient follow-up period ( $n=1$ : Mansouri 2006);

- not utilising a professional periodontal intervention ( $n=4$ : $\mathrm{Al}$ Mubarak 2002 (irrigation device); Cinar 2014 (empowerment "health coaching"); Gorbacheva 2010 (toothpaste); Khader 2010 (full mouth tooth extraction));

- abandoned due to recruitment issues ( $n=1$ : NCT01255254);

- a study previously classified under Ongoing studies in the 2010 version of this review has now been excluded, as reinspection of abstract indicates inclusion of non-diabetic controls (Hagiwara 2002).

\section{Studies awaiting classification}

Five studies are awaiting classification, and are fully detailed under Characteristics of studies awaiting classification.

Clarification is required for five of the studies to complete their assessment (relating to periodontitis diagnosis: Al-Mubarak 2010; Chee 2006; Lin 2012; or relating to poor reporting: Botero 2013; Nassar 2014). For all five studies, contact authors were emailed to request required information, although responses were not received prior to publication of this review update. We will reattempt classification of these five studies when this review is next updated.

\section{Ongoing studies}

Twelve studies are still ongoing and detailed under Characteristics of ongoing studies.

\section{Risk of bias in included studies}

Four teams of two authors independently assessed risk of bias for each included study. For consistent rating application, two authors (Jo Weldon (JW) and Zipporah Iheozor-Ejiofor (ZIE)) arbitrated these assessments in accordance with guidance from Chapter 8 of the Cochrane Handbook for Systematic Reviews of Interventions Version 5.1.0 (updated March 2011) (Higgins 2011). All except six of the studies were judged to be at high risk of bias.Two studies (Miranda 2014; Santos 2013) were low risk and four studies (Haerian Ardakani 2014; Li 2011; Macedo 2014; Pradeep 2013) were unclear.

A 'Risk of bias' table was completed for each included study (Characteristics of included studies). Results are presented graphically by study (Figure 2) and by domain across all studies (Figure 3). 
Figure 2. Risk of bias summary: review authors' judgements about each risk of bias item for each included study

\begin{tabular}{|c|c|c|c|c|c|c|c|}
\hline & 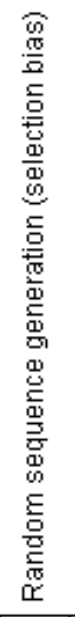 & 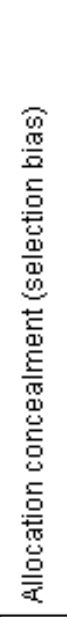 & 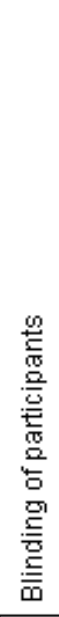 & 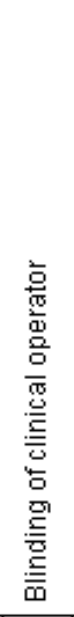 & 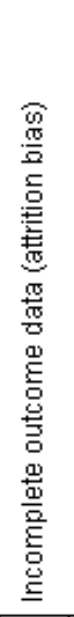 & 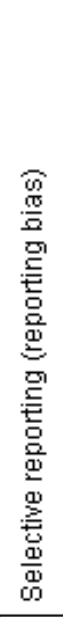 & 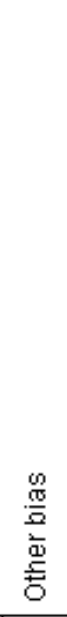 \\
\hline Al-Zahrani 2009 & + & $?$ & - & $\Theta$ & $?$ & + & + \\
\hline Calbacho 2004 & + & $?$ & - & $\odot$ & $\odot$ & $\odot$ & $?$ \\
\hline Chen 2012 & + & $?$ & $\odot$ & $?$ & $\odot$ & 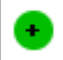 & + \\
\hline Engebretson 2011 & + & + & + & $?$ & $\odot$ & $\odot$ & + \\
\hline Engebretson 2013 & + & + & $\odot$ & 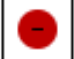 & + & 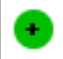 & $?$ \\
\hline Gay 2014 & + & $?$ & $?$ & $?$ & $\odot$ & $?$ & + \\
\hline Gilowski 2012 & + & + & + & + & $?$ & $\odot$ & + \\
\hline Grossi 1997 & $?$ & $?$ & $?$ & $\odot$ & $\odot$ & $?$ & - \\
\hline Haerian Ardakani 2014 & $?$ & $?$ & $?$ & $?$ & $\odot$ & $?$ & $?$ \\
\hline Jones 2007 & + & + & 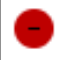 & $\odot$ & $\odot$ & $\odot$ & O \\
\hline Katagiri 2009 & $?$ & $\Theta$ & - & $\odot$ & $\odot$ & $?$ & + \\
\hline Kiran 2005 & + & + & - & $\odot$ & $\odot$ & $?$ & + \\
\hline Koromantzos 2011 & + & + & - & $\Theta$ & + & $?$ & + \\
\hline Kothiwale 2013 & $?$ & $?$ & - & $O$ & - & $?$ & $?$ \\
\hline Li 2011 & $?$ & $?$ & $?$ & $?$ & $?$ & $?$ & $?$ \\
\hline Llambés 2008 & $?$ & $?$ & - & $?$ & $\odot$ & - & + \\
\hline Macedo 2014 & + & $?$ & $?$ & $?$ & $\odot$ & + & + \\
\hline Madden 2008 & + & $?$ & $\odot$ & $?$ & $\theta$ & $\theta$ & $?$ \\
\hline Miranda 2014 & + & + & + & $\odot$ & $\odot$ & 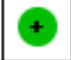 & + \\
\hline Moeintaghavi 2012 & + & $?$ & $\odot$ & $O$ & $\odot$ & $?$ & + \\
\hline NCT00801164 & + & $?$ & + & $?$ & $\ominus$ & $?$ & $?$ \\
\hline O'Connell 2008 & $?$ & $?$ & $?$ & $?$ & $\theta$ & $?$ & $\odot$ \\
\hline
\end{tabular}


Figure 2. (Continued)

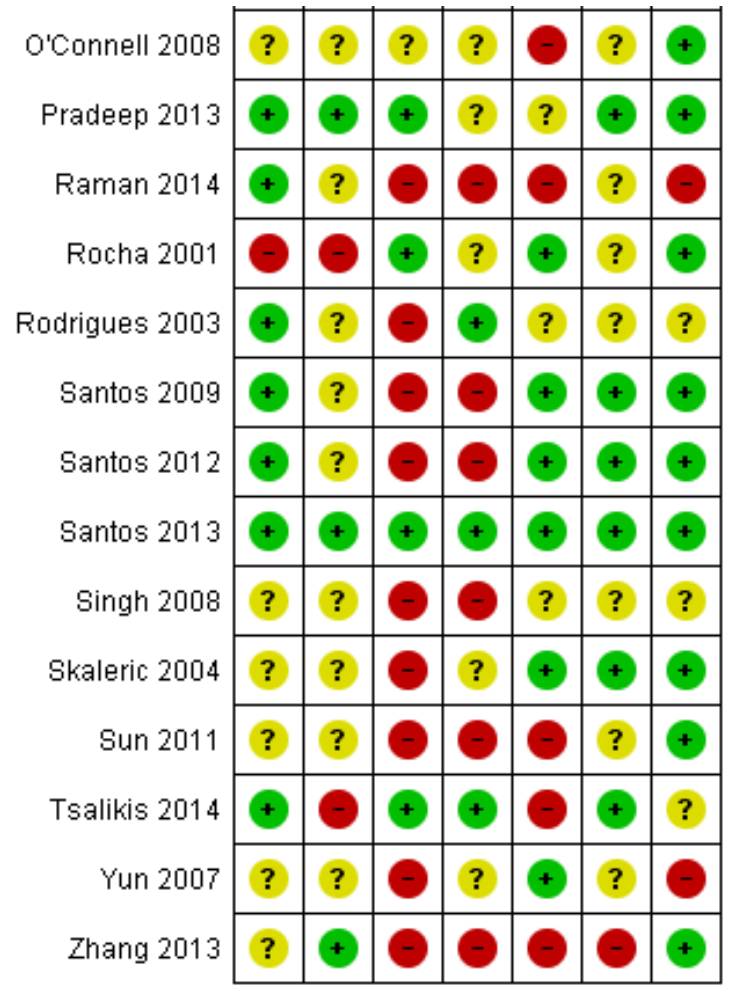

Figure 3. Risk of bias graph: review authors' judgements about each risk of bias item presented as percentages across all included studies

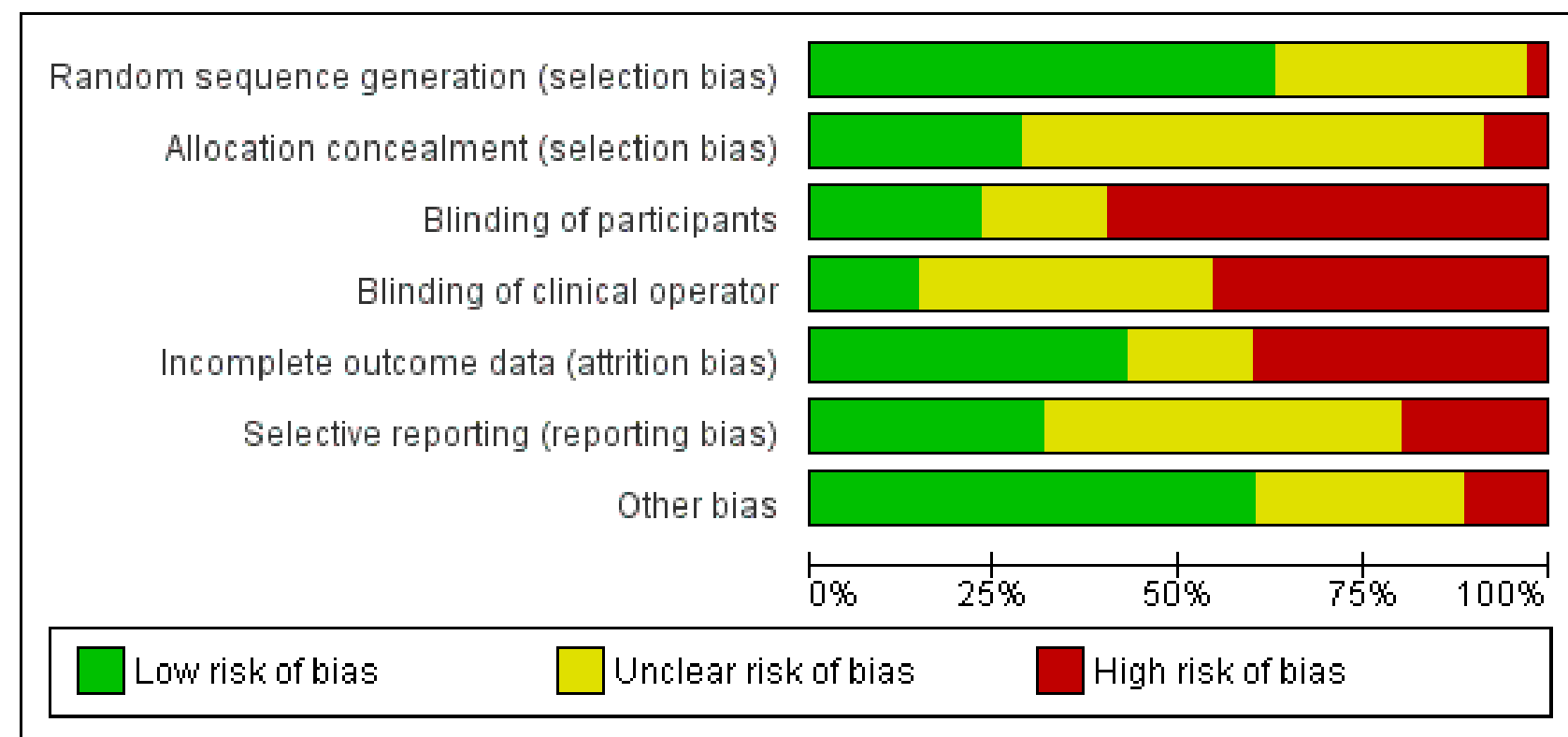

\section{Allocation}

We judged studies for selection bias based on the adequacy of random sequence generation and allocation concealment (to prevent selective enrolment).
Nine studies (Engebretson 2011; Engebretson 2013; Gilowski 2012; Jones 2007; Kiran 2005; Koromantzos 2011; Miranda 2014; Pradeep 2013; Santos 2013) reported adequate random sequence generation and allocation concealment and so were judged to be at low risk of selection bias. 
Three studies (Katagiri 2009; Rocha 2001; Tsalikis 2014) were judged to be at high risk of selection bias. This was due to dentists' knowledge of allocation (Katagiri 2009) and staff being in possession of the randomisation list (Tsalikis 2014). One study (Rocha 2001) reported insufficient information on randomisation, but was assumed to be quasi-randomised.

Twenty-three studies were judged to be at unclear risk of selection bias. This was mostly due to insufficient information about allocation concealment (Al-Zahrani 2009; Calbacho 2004; Chen 2012; Gay 2014; Macedo 2014; Madden 2008; Moeintaghavi 2012; NCT00801164; Raman 2014; Rodrigues 2003; Santos 2009; Santos 2012), randomisation (Zhang 2013) or both (Grossi 1997; Haerian Ardakani 2014; Kothiwale 2013; Li 2011; Llambés 2008; O'Connell 2008; Singh 2008; Skaleric 2004; Sun 2011; Yun 2007).

\section{Blinding}

\section{Performance bias}

We assessed studies for performance bias based on blinding of participants and clinical operators.

We judged four studies (Gilowski 2012; Miranda 2014; Santos 2013; Tsalikis 2014) to be at low risk of performance bias. Of the four studies, three studies (Gilowski 2012; Santos 2013; Tsalikis 2014) used placebo, and personnel were reported to have been blinded or unaware of treatment. One study (Miranda 2014), in addition to blinding of the participants and personnel, also reported that code breaking was only performed after the final analysis.

Nine studies (Engebretson 2011; Gay 2014; Haerian Ardakani 2014; Li 2011; Macedo 2014; NCT00801164; O'Connell 2008; Pradeep 2013; Rocha 2001) were at unclear risk of performance bias. This was either because the studies were referred to as double blinded without further details, or blinding would have been possible but was not reported.

We judged 22 studies to be at high risk of bias of performance bias. Bias was due lack of participant blinding (Chen 2012; Llambés 2008; Madden 2008; Rodrigues 2003; Skaleric 2004; Yun 2007), lack of personnel blinding (Grossi 1997), or both (Al-Zahrani 2009; Calbacho 2004; Engebretson 2013; Jones 2007; Katagiri 2009; Kiran 2005; Koromantzos 2011; Kothiwale 2013; Moeintaghavi 2012; Raman 2014; Santos 2009; Santos 2012; Singh 2008; Sun 2011; Zhang 2013). The studies at high risk of performance bias provided information indicating that blinding was not undertaken at all, was unachievable due to nature of intervention, or was attempted but broken.

\section{Detection bias}

We did not assess the studies for detection bias as $\mathrm{HbAlc}$ tests were carried out remotely, therefore all studies were considered to be at low risk. Blind outcome assessment for the secondary outcomes was considered to be of lesser importance so as not to detract from the primary focus of the review.

\section{Incomplete outcome data}

We judged 15 studies (Calbacho 2004; Engebretson 2013; Haerian Ardakani 2014; Katagiri 2009; Kiran 2005; Koromantzos 2011; Macedo 2014; Miranda 2014; Moeintaghavi 2012; Rocha 2001; Santos 2009; Santos 2012; Santos 2013; Skaleric 2004; Yun 2007) to be at low risk of incomplete outcome bias. This assessment was due to studies reporting 100\% completion (Calbacho 2004; Haerian Ardakani 2014; Katagiri 2009; Kiran 2005; Koromantzos 2011; Macedo 2014; Moeintaghavi 2012; Santos 2009; Santos 2012; Skaleric 2004), similarly low attrition rates across groups (Engebretson 2013; Miranda 2014; Santos 2013) in addition to conducting intention-to-treat (ITT) analysis. One study (Yun 2007) did not provide sufficient information, but appears not to have had any drop-outs. Rocha 2001 was considered to be ITT due to indicated degrees of freedom reported in paired t-test.

We judged six studies to be at unclear risk of incomplete outcome bias. This judgement was based on lack of sufficient information on attrition even though ITT analysis is assumed to have been undertaken (Al-Zahrani 2009; Gilowski 2012; Pradeep 2013; Rodrigues 2003; Singh 2008). One study reported no information ( $\mathrm{Li}$ 2011).

Fourteen studies were at high risk of incomplete outcome bias. Though ITT analysis was undertaken in one study (Engebretson 2011), the attrition rate of over $20 \%$ was still considered a source of bias. The rest of the studies (Chen 2012; Gay 2014; Grossi 1997; Jones 2007; Kothiwale 2013; Llambés 2008; Madden 2008; NCT00801164; O'Connell 2008; Raman 2014; Sun 2011; Tsalikis 2014; Zhang 2013) also reported withdrawals, but did not analyse all participants in the group to which they were randomised.

\section{Selective reporting}

We judged 11 studies (Al-Zahrani 2009; Chen 2012; Engebretson 2013; Macedo 2014; Miranda 2014; Pradeep 2013; Santos 2009; Santos 2012; Santos 2013; Skaleric 2004; Tsalikis 2014) to be at low risk of selective reporting bias as all expected outcome including adverse events were recorded and fully reported.

Seventeen studies were at unclear risk of selective reporting bias. Fourteen of these studies were judged to be unclear because there was no information on whether any adverse events had occurred or not (Gay 2014; Grossi 1997; Haerian Ardakani 2014; Katagiri 2009; Kiran 2005; Koromantzos 2011; Kothiwale 2013; Moeintaghavi 2012; O'Connell 2008; Raman 2014; Rocha 2001; Rodrigues 2003; Sun 2011; Yun 2007), and one study only partially reported adverse effects (Singh 2008: for an intervention component of a single group). For the remaining two studies (Li 2011; NCT00801164), there was insufficient information to make a judgement.

We judged seven studies to be at high risk of selective reporting bias. Some studies at high risk of bias either failed to report on periodontal outcome fully (Engebretson 2011; Gilowski 2012), or did not report both periodontal outcome and adverse events (Calbacho 2004; Llambés 2008). Authors of two studies that reported HbA1C data in subgroups (Madden 2008) and inconsistently (Zhang 2013) were contacted via email, but message delivery failed. One study (Jones 2007) reported on adverse events for one arm only and also did not present $\mathrm{HbAlc}$ data at 4-month follow-up.

\section{Other potential sources of bias}

We judged 21 studies (Al-Zahrani 2009; Chen 2012; Engebretson 2011; Gay 2014; Gilowski 2012; Katagiri 2009; Kiran 2005; Koromantzos 2011; Llambés 2008; Macedo 2014; Miranda 2014; Moeintaghavi 2012; O'Connell 2008; Pradeep 2013; Rocha 2001; Santos 2009; Santos 2012; Santos 2013; Skaleric 2004; Sun 2011; Zhang 2013) to be at low risk of other potential sources of bias. 
We judged 10 studies to be at unclear risk of other potential sources of bias. We were unable to verify whether there were any other apparent biases due insufficient information from data extraction components of translated (Haerian Ardakani 2014; Li 2011) and unpublished studies (Calbacho 2004; Kothiwale 2013; NCT00801164). It was not clear whether there was baseline balance since participant characteristics were not reported (Madden 2008; Rodrigues 2003; Singh 2008). In another study, conflict of interests were reported for all authors except the lead author (Engebretson 2013). One study did not report the proportion of participants in receipt of hypoglycaemic medications (Tsalikis 2014).

We judged four studies to be at high risk of other potential sources of bias. There was baseline imbalance due to the randomisation of more participants with poor metabolic control to the intervention group (Raman 2014) and a difference between groups in participant characteristics (Grossi 1997; Jones 2007). One study (Yun 2007) was judged to be at high risk of bias due to overall poor reporting and study design.

\section{Effects of interventions}

See: Summary of findings for the main comparison Periodontal therapy compared to no active intervention/usual care for glycaemic control in people with diabetes mellitus; Summary of findings 2 Periodontal therapy compared to alternative periodontal therapy for glycaemic control in people with diabetes mellitus

We investigated two comparisons in this review.
1. Does periodontal therapy improve glycaemic control in people with diabetes mellitus?

2. Does one periodontal therapy have a greater effect than another on improving glycaemic control?

\section{Comparison 1. Periodontal therapy versus no active intervention/usual care}

\section{HbA1c: 3-4 months (Analysis 1.1)}

Fourteen studies (1499 participants) compared periodontal therapy against no active intervention/usual care at 3 or 4 months. Overall, there was a benefit for periodontal therapy with a mean percentage reduction in $\mathrm{HbA1C}$ of -0.29 (95\% confidence interval $(\mathrm{Cl})-0.48$ to -0.10 ; effect $P=0.003)$. There was a moderate amount of heterogeneity $\left(P=0.008 ; 1^{2}=53 \%\right)$ (Analysis 1.1 ).

Two subgroups were formed for studies: scaling and root planing (SRP) (eight studies), and SRP plus antimicrobials (seven studies). One study (Singh 2008) contributed data to both subgroups. There was no statistically significant difference between the subgroups ( $P$ $=0.25$ ).

A funnel plot of the 15 included studies (Figure 4: reflecting Singh 2008's contribution to both subgroups) failed to indicate any relationship between mean percentage reduction in $\mathrm{HbA} 1 \mathrm{c}$ and precision (related to sample size). The Egger formal test for asymmetry intercept, was not statistically significant: $-0.94(95 \% \mathrm{Cl}$ -2.40 to $0.52 ; \mathrm{P}=0.19)$ (Egger 1997). 
Figure 4. Funnel plot of comparison: 1 Periodontal therapy versus no active intervention/usual care, outcome: 1.1 HbA1c at 3-4 months

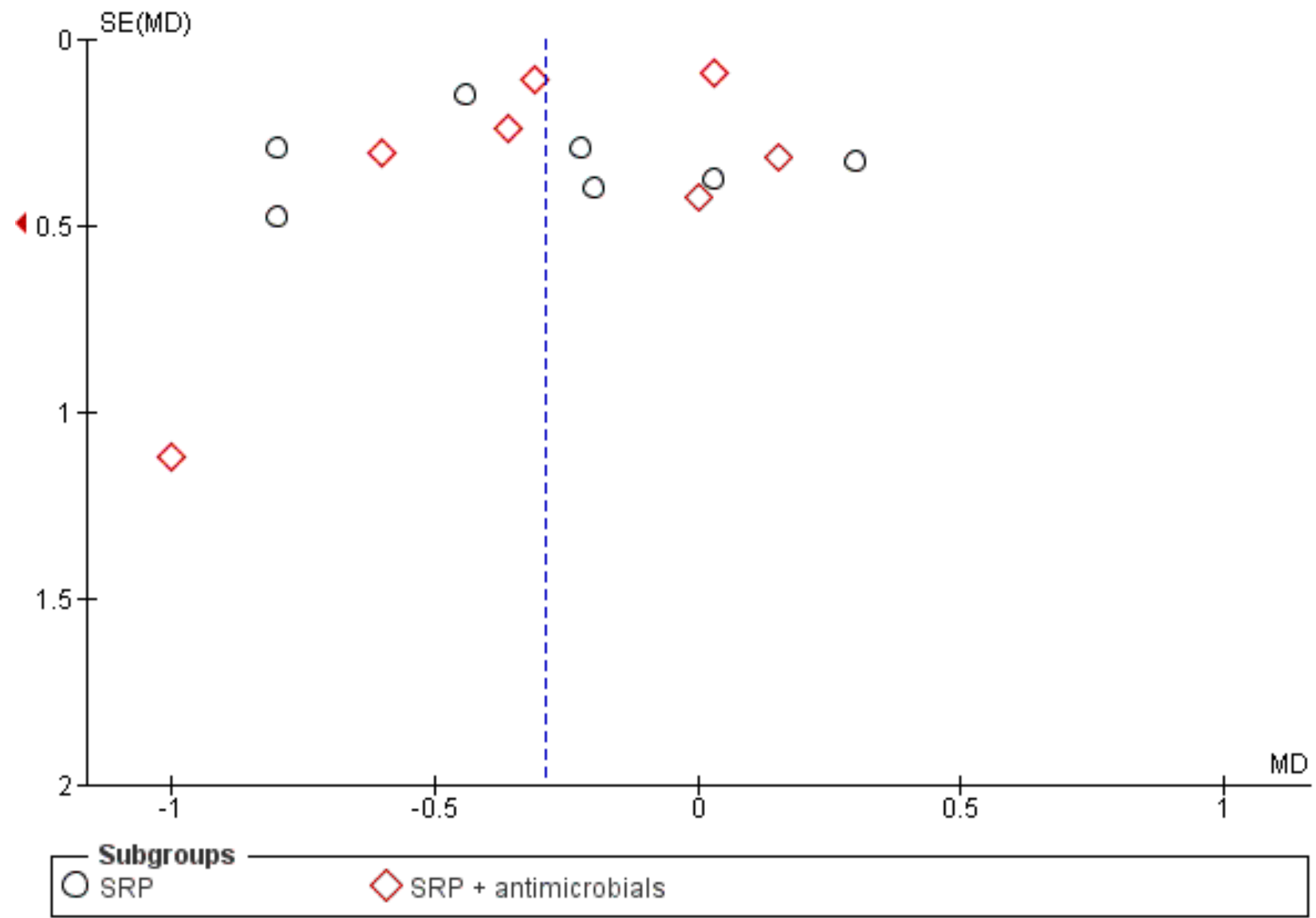

\section{HbA1c: 6 months (Analysis 1.2)}

Five studies (826 participants) compared periodontal therapy against no active intervention/usual care at 6 months. Overall, there was no benefit for periodontal therapy with mean percentage reduction in $\mathrm{HbA} 1 \mathrm{C}$ of $-0.02(95 \% \mathrm{Cl}-0.20$ to 0.16 ; effect $\mathrm{P}=0.84)$. There was little evidence of heterogeneity $\left(P=0.69 ; 1^{2}=0 \%\right)$.

Two subgroups were formed for studies: SRP (three studies), and SRP plus antimicrobials (two studies). There was no statistically significant difference between the subgroups $(P=0.38)$ (Analysis 1.2).

\section{Comparison 2. Periodontal therapy versus alternative periodontal therapy}

The different interventions being compared varied across studies, and are clearly detailed in Additional Table 5 within this review.

\section{HbA1c: 3-4 months (Analysis 2.1)}

Twenty-one studies (920 participants) compared different periodontal therapies at 3 or 4 months, with adjunctive intervention being given in one or both groups.

The studies were categorised into the following detailed comparisons:
- SRP versus alternative mechanical therapy (one study)

- SRP versus alternative SRP (three studies)

- SRP plus antimicrobial versus antimicrobial (one study)

- SRP plus antimicrobial versus SRP (12 studies)

- SRP plus antimicrobial (doxycyline) versus SRP plus alternative antimicrobial (three studies)

- SRP plus combined antimicrobials versus SRP plus alternative antimicrobial (three studies)

- SRP plus statin versus SRP (one study).

We were unable to pool most of these head-to-head comparisons.

We were able to pool the SRP plus antimicrobial versus SRP comparison. There was no consistent evidence that the addition of antimicrobials to SRP was of any benefit to delivering SRP alone (mean HbA1c 0.00\% lower: 12 studies, 450 patients; $95 \% \mathrm{Cl} 0.22 \%$ lower to $0.22 \%$ higher) at 3 or 4 months post-treatment. There was little evidence of heterogeneity $\left(P=0.16 ; 1^{2}=29 \%\right)$.

\section{Sensitivity analysis}

A sensitivity analysis, based on restricting the meta-analysis of the pooled SRP plus antimicrobial versus SRP subgroup to the two studies assessed as being at low risk of bias indicated that the result is robust in stating that there was no consistent evidence that the 
addition of antimicrobials to SRP was of any benefit to delivering SRP alone.

A funnel plot of the 12 included studies in analysis 2.1.4 (Figure

5) failed to indicate any relationship between mean percentage reduction in $\mathrm{HbAlc}$ and precision (related to sample size). The Egger formal test for asymmetry intercept, was not statistically significant: $0.12(95 \% \mathrm{Cl}-1.33$ to $1.57 ; \mathrm{P}=0.86)$ (Egger 1997).

Figure 5. Funnel plot of comparison: 2 Periodontal therapy versus alternative periodontal therapy, outcome: 2.1 HbA1c at 3-4 months

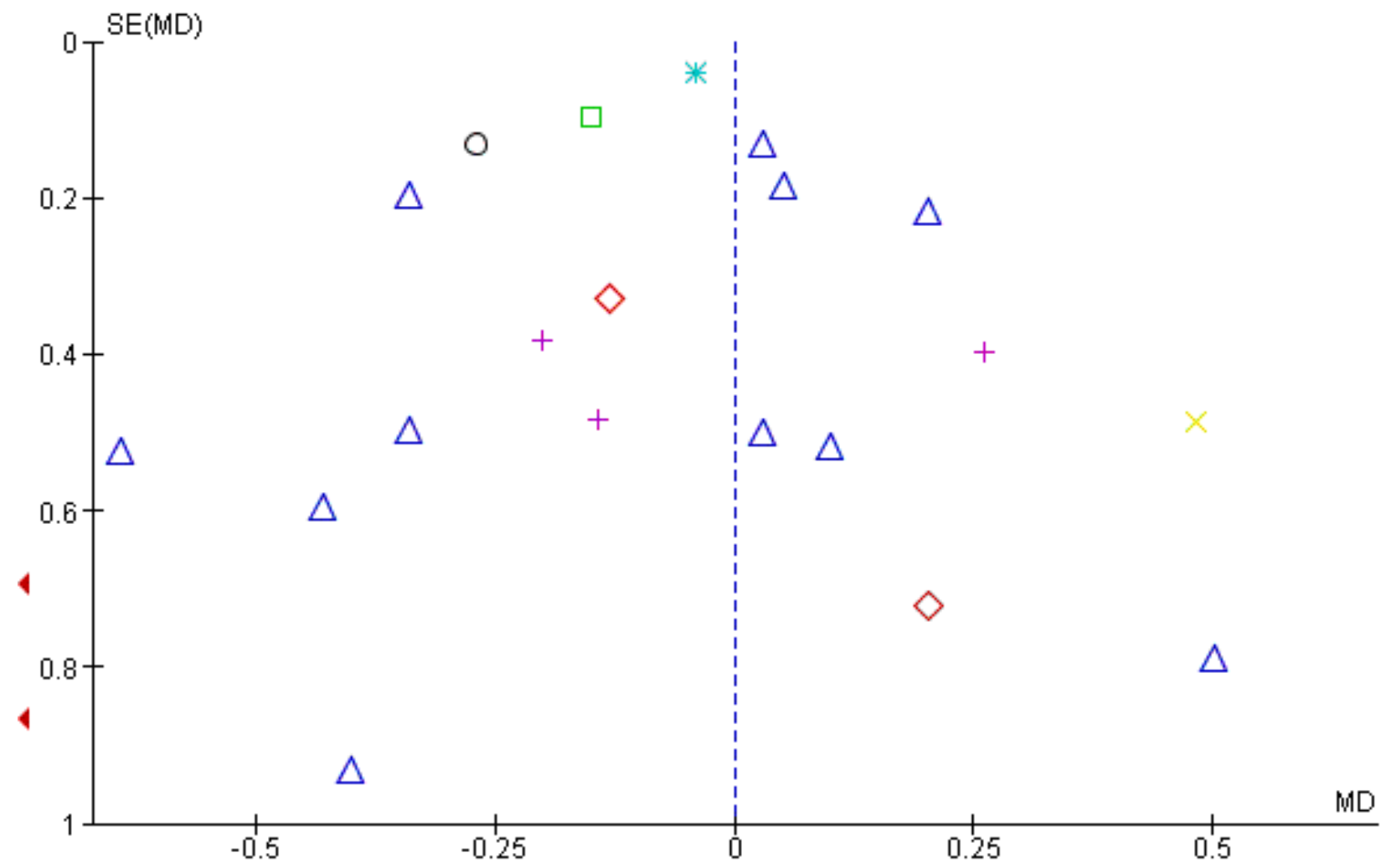

Subgroups

SRP vs alternative mechanical therapy

SRP vs alternative SRP

SRP + antimicrobial vs antimicrobial

SRP + antimicrobial vs SRP

SRP + antimicrobial (doxycycline) vs SRP + alternative antimicrobial

+ SRP + combined antimicrobials vs SRP + single antimicrobial

SRP + statin vs SRP

\section{HbA1c: 6 months (Analysis 2.2)}

Twelve studies (34\%; 583 participants) compared periodontal therapy against an alternative periodontal therapy at 6 months, with adjunctive intervention being given in one or both groups. It was inappropriate to combine studies for estimation of an overall pooled effect across subgroups.

The studies were categorised into the following detailed comparisons:

- SRP versus alternative mechanical therapy (one study)

- SRP versus alternative SRP (three studies)

- SRP plus antimicrobial versus SRP (five studies)
- SRP plus antimicrobial (doxycyline) versus SRP plus alternative antimicrobial (one study)

- SRP plus combined antimicrobials versus SRP plus alternative antimicrobial (one study)

- SRP plus bone modifier versus SRP (one study)

- SRP plus statin versus SRP (one study).

We were unable to pool most of these head-to-head comparisons.

Once again we were able to pool the SRP plus antimicrobial versus SRP comparison. There was no consistent evidence that the addition of antimicrobials to SRP was of any benefit to delivering SRP alone (mean HbA1c 0.04\% lower: five studies, 206 patients; 
$95 \% \mathrm{Cl} 0.41 \%$ lower to $0.32 \%$ higher) at 6 months post-treatment. There was little evidence of heterogeneity $\left(P=0.11 ; 1^{2}=46 \%\right)$ (Analysis 2.2).

\section{Sensitivity analysis}

A sensitivity analysis, based on restricting the meta-analysis of the pooled SRP plus antimicrobial versus SRP comparison to the only two studies assessed as being at low risk of bias indicated that the result is robust in stating that there was no consistent evidence that the addition of antimicrobials to SRP was of any benefit to delivering SRP alone.

\section{Secondary outcomes}

\section{Periodontal indices}

To allow direct comparison of data from studies on the same scale, measurements were converted to the proportion of sites for the following secondary (periodontal parameter) outcomes: bleeding on probing (BOP) (Chen 2012; Engebretson 2013; Gilowsk 2012; Katagiri 2009; Kiran 2005; Koromantzos 2011; Macedo 2014; O'Connell 2008; Rodrigues 2003; Santos 2009; Santos 2012; Santos 2013; Yun 2007), gingival index (GI) (Grossi 1997; Koromantzos 2011), and plaque index (PI) (Gilowski 2012; Grossi 1997; Haerian Ardakani 2014; Macedo 2014; Moeintaghavi 2012; O'Connell 2008; Santos 2009; Santos 2012; Santos 2013).

\section{Comparison 1. Periodontal therapy versus no active intervention/ usual care}

Additional Table 6 documents the clinical periodontal secondary outcomes for the studies included in this comparison at both 3 to 4 , and 6 months.

Statistically significant differences in favour of periodontal intervention were found for all periodontal indices (BOP, clinical attachment level (CAL), GI, PI, probing pocket depth (PPD)) at both 3 to 4 , and 6 months follow-up.

\section{Comparison 2. Periodontal therapy versus alternative periodontal therapy}

Additional Table 7 documents the clinical periodontal secondary outcomes for the studies included in this comparison at both 3 to 4 , and 6 months. The success of the periodontal interventions compared are variable across clinical indices and time points.

\section{Adverse effects}

Twenty studies (57\%) did not report whether their participants experienced adverse effects from their allocated treatment (Calbacho 2004; Gay 2014; Grossi 1997; Haerian Ardakani 2014; Katagiri 2009; Kiran 2005; Koromantzos 2011; Kothiwale 2013; Li 2011; Llambés 2008; Madden 2008; Moeintaghavi 2012; NCT00801164; O'Connell 2008; Raman 2014; Rocha 2001; Rodrigues 2003; Sun 2011; Yun 2007; Zhang 2013).

- Eight studies $(23 \%)$ reported that no adverse events occurred (Al-Zahrani 2009; Chen 2012; Engebretson 2011; Gilowski 2012; Macedo 2014; Pradeep 2013; Santos 2009; Santos 2012).

- One study $(3 \%)$ reported that no adverse effects resulted from use of doxycycline (Singh 2008 as partial intervention component for $\mathrm{Gp} \mathrm{B}$ ), but failed to report whether any side effects resulted from SRP or were experienced by Gps A and C.
- Three studies (9\%) reported that no major adverse events occurred (Engebretson 2013 (common SRP discomfort only); Skaleric 2004; Tsalikis 2014 (one control patient reported dizziness/swallowing difficulty))

- Three studies (9\%) reported the occurrence of minor adverse events (Jones 2007 (doxycyline: diarrhoea, pain, nausea; chlorhexidine: taste change, tooth stain, mouth irritation, swelling and breathlessness); Miranda 2014 (both groups: diarrhoea, headaches, metallic taste, nausea/vomiting); Santos 2013 (more than half in both groups reported taste perception change/dry mouth/staining)).

\section{Quality of life}

No included studies reported data relating to quality of life.

\section{Cost implications}

No included studies reported data relating to cost implications.

\section{Diabetic complications}

No included studies reported data relating to diabetic complications.

\section{ISC U S S I O N}

\section{Summary of main results}

The primary objective was to investigate the effect of periodontal therapy on glycaemic control in people with diabetes. We found evidence to demonstrate that the treatment of periodontal disease does improve glycaemic control in people with diabetes, with a mean percentage reduction of $0.29 \%$ in $\mathrm{HbAlc}$ at 3-4 months. The quality of the body of evidence for this finding was assessed as low.

To put this in context, the epidemiological analysis of UKPDS 1998 data indicated that for every percentage point decrease in $\mathrm{HbA1c}$, there was a $35 \%$ reduction in the risk of microvascular complications, which appeared to be linear; however, we acknowledge that a linear relationship may not exist at lower levels. In a general population, of whom only a minority had diabetes, a lower average $\mathrm{HbA} 1 \mathrm{c}$ level by $0.2 \%$ was associated with a $10 \%$ lower mortality (Khaw 2001) over 2-5 years. although the findings of this and other observational studies of people with diabetes are prone to confounding.

Glycaemic control is only one component of management of diabetes and smoking cessation, weight loss, physical activity and management of dyslipidaemia and hypertension, where appropriate, are particularly important to reduce risk of macrovascular disease. Consequently, the effect of the modest reduction $(0.2 \%)$ in $\mathrm{HbAlc}$ seems unlikely to result in a major population-level effect, particularly as effectiveness of periodontal treatment is only estimated to 3-4 months post-treatment within this review.

There was no evidence that periodontal treatment results in a significant effect at 6 months. Due to periodontitis being a chronic condition, ongoing maintenance care by clinicians would be prudent to maintain clinical improvements beyond 6 months.

Further research is still needed to determine whether or not periodontal treatment (with or without adjunctive intervention) provides further benefit with regard to glycaemic control 
beyond other interventions of known effectiveness including lifestyle and behavioural change and treatment intensification, and perhaps future randomised controlled trials (RCTs) could look at this. Interestingly, another systematic review's metaanalysis (including 19 RCTs, 1431 participants) demonstrated that psychological intervention (counselling, cognitive behavioural therapy or psychodynamic therapy) results in a mean difference (MD) reduction in $\mathrm{HbAlC}$ of $0.54 \%$ (95\% confidence interval $(\mathrm{Cl})$ -0.83 to -0.23 ) for type 2 diabetes mellitus (T2DM) patients (Alam 2009). While their review is not directly comparable (participants were not diagnosed with periodontitis, and their results pooled the effects of RCTs across a broad range of follow-up periods (4 weeks to 12 months), we highlight that future trials could potentially also consider the use of adjunctive psychological intervention as this may further improve the reduction in $\mathrm{HbA} 1 \mathrm{c}$ of $0.29 \%$ demonstrated in this review, but that such a design will require quality of life (QoL) to be measured as an outcome within such trials. Trials may be designed to include three arms, including a no periodontal treatment control.

We also wish to highlight that due to the array of associated factors (eg QoL, diabetic complications) that have been shown to influence glycaemic control, establishing a direct relationship may be particularly difficult (Chew 2015). Future trials might attempt to address this issue by including the use of health-related QoL assessments and more thoroughly reporting the prevalence of diabetic complications from their sample population.

There was no evidence of a difference in glycaemic control demonstrated by delivery of different periodontal treatments (comparison 2 in this review).

There was some variation between studies of the effect of interventions in achieving periodontal health, with some showing high levels of residual inflammation following treatment. For the trials comparing the effect of periodontal therapy with a usual care or no active treatment control group, there was evidence of reductions for all clinical periodontal indices at both 3-4 and 6 months for patients in the periodontal therapy group.

There were several head-to-head studies comparing different periodontal treatments. There was no clear evidence that one treatment was more effective than another.

\section{Overall completeness and applicability of evidence}

The review assessed a varied population with a wide age range, good gender balance and varied glycaemic control (HbAlc thresholds) who were using different forms of antidiabetic therapy; however, information about socioeconomic status (SES) and body mass index (BMI) were rarely reported, raising doubts concerning the applicability of the evidence to people of various sizes and socioeconomic backgrounds.

The studies included in the meta-analyses all provided $\mathrm{HbAlc}$ data, however, adverse events were seldom reported. Participation in the trials might have resulted in patients monitoring their blood sugars and taking better care of their health, by complying with their medication more than they normally would. This might have resulted in an overestimation of the benefit of periodontal interventions due to potential Hawthorne effect impact (McCambridge 2014).
Overall, we find the evidence presented in this review to be pragmatic as the results of most of the studies were analysed by intention-to-treat (ITT); however, there was some variation between studies of the effect of interventions in achieving periodontal health, with some showing high levels of residual inflammation following treatment. As the postulated effect of scaling and root planing (SRP) on glycaemic control is predicated upon reduced periodontal inflammation, reduced efficacy of the intervention may have restricted the potential to show benefit.

A recent cross-sectional survey (Kowall 2015) concluded that having pre-diabetes or well-controlled T2DM (defined in their study as a known $\mathrm{HbA1}$ c level of $<7.0 \%$ ) was not associated with a greater prevalence of periodontitis or edentulism; however, their paper implies an association exists for people with $\mathrm{HbA1c}$ levels greater than $7.0 \%$.

Considering the implications of their findings, we highlight an RCT (Khader 2010) excluded by our own review due to its use of a non-periodontal intervention, full mouth tooth extraction (FME). This study, of 58 T2DM participants diagnosed with severe periodontitis, excluded patients with a baseline $\mathrm{HbA1c}$ level up to $7.0 \%$ (being considered to have well-controlled T2DM), and randomised included participants (all of whose remaining teeth were deemed to be in a hopeless condition and indicated for extraction regardless) to receive FME or no treatment (delayed FME). Khader 2010's choice of intervention potentially addresses our hypothesis in a situation where all periodontal disease may be presumed to be eliminated by use of FME. Significant reductions in mean $\mathrm{HbA} 1 \mathrm{c}$ were demonstrated by the intervention group (FME recipients) at both 3 months follow-up (MD $-0.95 \%$; 95\% Cl 1.41\% lower to $0.49 \%$ lower), and 6 months follow-up also (MD -1.09\%; $95 \% \mathrm{Cl} 1.57 \%$ lower to $0.61 \%$ lower). The improvement detected in this study suggests that the benefits of eliminating infection or inflammation associated with periodontal disease in people with T2DM are likely to be centred on those with both poor metabolic control and with the most severe presentation of periodontal disease.

\section{Quality of the evidence}

The quality of the body of evidence was low due to high risk of bias, moderate to substantial heterogeneity and serious imprecision.

All the included studies were at high risk of bias except six studies, two $(6 \%)$ at low risk of bias and four (11\%) at unclear risk of bias. Bias was due to inadequate randomisation/allocation concealment in four $(11 \%)$ studies, selective reporting in seven (20\%) studies and attrition in $14(40 \%)$ studies. However, the main source of bias was lack of blinding of participants and clinical operator which led to performance bias in $22(63 \%)$ studies.This occurred in studies involving interventions that were mechanical in nature, for which blinding was not possible.

There was little evidence of heterogeneity across the studies despite differences in intervention delivery and various treatment combinations. There was no indirectness nor publication bias detected.

When compared to usual care/no active treatment, different periodontal treatments showed consistently significant effects at 3-4 months follow-up. This significant effect was fairly consistent 
regardless of intervention type and supports confidence in the estimate of effect.

\section{Potential biases in the review process}

Concerted efforts have been made to prevent bias arising in the review process:

- by cross-checking references from existing literature to ensure previously identified studies by others were appraised for inclusion in this review in the eventuality that this review's search strategy had not highlighted them;

- by screening search result records in duplicate and having all decisions arbitrated by a third review author;

- by adherence to an agreed, standardised data extraction format, utilised by at least two review authors per included study and contents reviewed for accuracy/consistency with the original record/s by an additional author;

- by attempting to contact authors (not all attempts were successful) of included/ongoing/excluded studies to obtain/ verify additional information missing from publications/records arising from their studies; and

- by use of standard Cochrane tools to utilise as much data as possible from included studies within the two comparisons in this review (eg by combining intervention arms, or splitting control group patients, from multiarmed studies, where appropriate).

Despite these attempts to avoid bias, there were other sources of potential bias in the review process which we were unable to prevent. Data extraction and risk of bias assessment were done by different teams and may have led to inconsistent ratings; however, two review authors (Jo Weldon (JW) and Zipporah Iheozor-Ejiofor (ZIE)) arbitrated all assessments in order to maintain uniformity.

We chose not to incorporate assessment of periodontal outcome detection bias as it was not the primary focus of the review. We highlight that change scores were used in meta-analysis for the following secondary (periodontal parameter) outcomes: bleeding on probing (BOP) (Engebretson 2013), clinical attachment level (CAL) (Grossi 1997; Li 2011), gingival index (GI) (Engebretson 2013), plaque index (PI) (Li 2011), and probing pocket depth (PPD) (Li 2011); however, this is indicated in the Cochrane Handbook for Systematic Reviews of Interventions Version 5.1.0 (updated March 2011) (Higgins 2011: section 9.4.5.2) as an acceptable method and consequently is not a concern for bias.

We imputed standard deviations (SDs) from reported means for BOP, CAL, GI and PPD (derived from reported Cls) by using standard Cochrane tools for Engebretson 2013.

Where we were unable to contact study authors to verify information, we will reattempt contact at the next update to ensure inclusion/accurate reporting of as many relevant studies as possible.

\section{Agreements and disagreements with other studies or reviews}

Three other recent systematic reviews (Engebretson 2013a; Sgolastra 2013; Teeuw 2010) evaluated the effect of periodontal treatment with no treatment (Comparison 1 in this review) in reducing $\mathrm{HbAlc}$ for patients with diabetes.
After 3 months, Teeuw 2010 (five included studies, 371 participants) estimated weighted mean $\mathrm{HbAlc}$ was lower by $0.40 \%(95 \% \mathrm{Cl}$ -0.77 to $-0.04 \% ; \mathrm{P}=0.03$ ), Sgolastra 2013 (five included studies, 315 participants) estimated weighted mean $\mathrm{HbA1c}$ was lower by $0.65 \%$ ( $95 \% \mathrm{Cl} 0.43$ to $0.88 ; \mathrm{P}<0.05$ ), and Engebretson 2013a (nine included studies, 719 participants) found $\mathrm{HbAlC}$ was reduced by $0.36 \%(95 \% \mathrm{Cl}-0.54$ to $-0.19 ; \mathrm{P}<0.0001)$. This review's relevant meta-analysis (14 included studies, 1499 participants) estimates weighted mean $\mathrm{HbAlc}$ to be $0.29 \%$ lower $(95 \% \mathrm{Cl}-0.48$ to -0.10 ; $\mathrm{P}=$ 0.003) after 3-4 months.

As would be expected with an increased number of included studies and participants, the treatment effect estimate range has narrowed from previous reviews.

We concur with author reported limitations from Engebretson 2013a; Sgolastra 2013 and Teeuw 2010 that intervention variety, small sample size of included studies/lack of large-scale RCTs, and an inherent high risk of bias (owed largely to the impossibility of blinding patients and operators to group allocation for many interventions) may impact the estimation of accurate treatment effects from periodontal intervention when compared with no active treatment; however, we must acknowledge that the inclusion of Engebretson 2013 (accounting for 34\% of participants in comparison 1) significantly increased the sample size available for analysis.

Due to the large number of participants contributed by Engebretson 2013, it is appropriate to address some specific concerns about the trial raised in other literature (Borgnakke 2014) regarding included patients' HbAlc range being close to optimal levels, the decision to include patients above and below protocol-specified thresholds and high BMI. Having considered these concerns, we believe that the inclusion of such patients reflects the breadth of population likely to be seen in clinical practice, and the inclusion of the trial enhances estimation of the true effect of periodontal treatment for glycaemic control in diabetic patients.

A further criticism of Engebretson 2013 made by Borgnakke 2014 is that the trial's periodontal outcomes indicate that the accepted standard of care was not met by the periodontal therapy provided to trial participants; however, in meta-analysis to derive treatment effect estimates for periodontal indices, Engebretson 2013's outcomes were consistent at both time points for all reported outcomes with the other included studies. Consequently, we are satisfied that Engebretson 2013's clinical conduct is not of sufficient concern to warrant post-hoc sensitivity analyses excluding its contribution, and have confidence in its findings being consistent with those of other included studies.

\section{AUTHORS' CONCLUSIONS}

\section{Implications for practice}

There is low quality evidence that the treatment of periodontal disease by scaling and root planing does improve glycaemic control in people with diabetes, with a mean percentage reduction in $\mathrm{HbA1C}$ of $0.29 \%$ at $3-4$ months; however, there is insufficient evidence to demonstrate that this is maintained after 4 months. 
There was no evidence to support that one periodontal therapy was more effective than another in improving glycaemic control in people with diabetes.

In clinical practice, ongoing professional periodontal treatment will be required to maintain clinical improvements beyond 6 months. Further research is required to determine whether adjunctive drug therapies should be used with periodontal treatment. Future randomised controlled trials (RCTs) should evaluate this, provide longer follow-up periods, and consider the inclusion of a third 'no treatment' control arm.

Larger, well conducted and clearly reported studies are needed in order to understand the potential of periodontal treatment to improve glycaemic control among people with diabetes. In addition, it will be important in future studies that the intervention is effective in reducing periodontal inflammation and maintaining it at lowered levels throughout the period of observation.

\section{Implications for research}

Further studies to confirm or refute these findings should be viewed as a public health priority in view of the prevalence of both periodontal disease and diabetes, and the potential impact of impaired diabetes management on morbidity, mortality and quality of life. We suggest a two-phase approach to investigating the importance of periodontal disease treatment on diabetes.

Firstly, a limited number of additional RCTs should be conducted investigating the impact of periodontal therapy on glycaemic control, with at least 6 months follow-up after treatment completion. Withholding treatment for the control group is not ethical. This could be managed, as with some of the reported studies, by the control group continuing with 'usual care' for the duration of the trial (eg Jones 2007: examples might include supragingival prophylaxis, standalone oral hygiene instruction or other reasonable oral care treatments), with rescue treatment provided for progressing periodontal disease. Trials should clearly report the occurrence of adverse events, and the use of antidiabetic therapy at baseline and changes to such therapy during the study periods (treatment phase and follow-up, ideally separately).

Each trial should be large enough to have sufficient power to demonstrate a statistically significant difference if it exists. The data from the existing trials should be able to provide data for such a calculation (allowing for losses to follow-up). Definitions of periodontal disease and diabetes should be clear, and studies should use the CONSORT statement to ensure adequate and transparent reporting of methodology. Trialists should also use the data from this systematic review and meta-analyses to inform their own study designs, and to share the results of new/ongoing trials to update collective findings and determine the overall totality of the evidence. In view of the many potential confounders that exist with a disease such as diabetes, study designs should also include data relating to age, duration, medication, smoking, $\mathrm{HbAlc}$ assessment standard (Diabetes Control and Complications Trial (DCCT) or the International Federation of Clinical Chemistry (IFCC)) and recognised diabetes-related complications.

The intervention and clinical setting should be appropriate for the severity of periodontitis (eg mild to moderate severity periodontitis should be treated in a similar way to primary or community care provision capabilities, whereas severe periodontitis may require specialist skills), and additional supportive maintenance care should be provided to the intervention group (as per usual practice) for studies in excess of 3 to 4 months duration. It will be important for future studies that the intervention is effective in both reducing periodontal inflammation, and maintaining it at lowered levels throughout the period of observation. In addition, studies should investigate the impact of periodontal therapy on both people with type 1 and type 2 diabetes mellitus, tobacco consumption status/ duration since smoking cessation, glycaemic control, and have sufficient included participants in each group to determine the treatment response in both groups.

Secondly, if the evidence clearly indicates a potentially clinically significant benefit, community-based trials should be conducted. Future research may involve close co-operative working between researchers with dental/periodontal and medical backgrounds. Such studies might include a variety of research designs, depending on the question to be addressed. For instance, multicentred and/ or international (including high income and low- to middle-income countries) RCTs (and possibly cluster-randomised trials) should be considered for addressing the question of the impact of periodontal therapy on control of diabetes. As well as glycaemic control, trials should measure quality of life and diabetes complications. Health economic evaluation is also important. Qualitative research could be used to address questions relating to barriers/facilitators of oral health and diabetes care, and preferences and experiences of patients. Furthermore, patient involvement in the design and conduct of these studies is strongly recommended.

\section{ACK N OWLEDGEMENTS}

We thank the Cochrane Oral Health Group (Cochrane OHG) for its support in undertaking this updated review: specifically, Anne Littlewood for running the searches; Laura MacDonald, Luisa Fernandez Mauleffinch and Anne-Marie Glenny for providing editorial and methodological comments; and Luisa Fernandez Mauleffinch for copy editing this review.

Our thanks to Rahul Alam, Deborah Matthews, Didac Mauricio and Hugo Pinto for peer-refereeing the clinical content of this review.

Additionally, we recognise the contribution from Cochrane OHG's translators: Chunjie Li (Li 2011), Andreas Neudecker (Albrecht 1988) and Farhad Shokraneh (Haerian Ardakani 2014; Mansouri 2006).

Particular thanks are due to the trial authors who provided unpublished information that allowed more thorough appraisal of studies: Mohammed Al-Zahrani (Al-Zahrani 2009), Mary Cullinan (ACTRN12605000260628; ACTRN12605000340639), Poliana Mendes Duarte (Miranda 2014; Santos 2009; Santos 2012), Elena Firkova (Yun 2007), Cristiane Miranda França (NCT01964833), Łukasz Gilowski (Gilowski 2012), Sara Grossi (Grossi 1997), Judith Jones (Jones 2007), Sayaka Katagiri (Katagiri 2009), Panagiotis Koromantzos (Koromantzos 2011), Shaila Patil Kothiwale (Kothiwale 2013), Fernando Llambés (Llambés 2008), Thomas Oates (NCT00801164), Rok Schara (Skaleric 2004), Jean Suvan (ISRCTN83229304), Mario Taba (Rodrigues 2003), Elif Unsal (Kiran 2005), and Jean-Noel Vergnes (Calbacho 2004; Mansouri 2006; ISRCTN15334496).

We would also like to acknowledge the contributions of Edward Mills to the original published review. 
Finally, particular thanks are expressed by Terry Simpson to Richard Ibbetson for his personal support and guidance during this review's initial conception. 


\section{R E F E R E N C E S}

\section{References to studies included in this review}

Al-Zahrani 2009 \{published and unpublished data\}

Al-Zahrani MS, Bamshmous SO, Alhassani AA, Al-Sherbini MM. Short-term effects of photodynamic therapy on periodontal status and glycemic control of patients with diabetes. Journal of Periodontology 2009;80:1568-73.

\section{Calbacho 2004 \{published and unpublished data\}}

Calbacho V, Carrasco E, Wilckens M, Barboza P, Grant C, Aguirre $\mathrm{M}$, et al. Evaluation of influence of conventional therapy in diabetics type 2. Journal of Dental Research 2004;84((Spec Iss B) Chilean section):65739.

\section{Chen 2012 \{published data only\}}

* Chen L, Luo G, Xuan D, Wei B, Liu F, Li J, et al. Effects of non-surgical periodontal treatment on clinical response, serum inflammatory parameters, and metabolic control in patients with type 2 diabetes: a randomized study. Journal of Periodontology 2012;83(4):435-43.

Chen L, Wei B, Li J, Liu F, Xuan D, Xie B, et al. Association of periodontal parameters with metabolic level and systemic inflammatory markers in patients with type 2 diabetes. Journal of Periodontology 2010;81(3):364-71.

\section{Engebretson 2011 \{published data only\}}

Engebretson SP, Hey-Hadavi J. Sub-antimicrobial doxycycline for periodontitis reduces hemoglobin Alc in subjects with type 2 diabetes: a pilot study. Pharmacological Research 2011; Vol. 64, issue 6:624-9.

\section{Engebretson 2013 \{published data only\}}

Engebretson S, Gelato M, Hyman L, Michalowicz BS, Schoenfeld E. Design features of the Diabetes and Periodontal Therapy Trial (DPTT): a multicenter randomized single-masked clinical trial testing the effect of nonsurgical periodontal therapy on glycosylated hemoglobin ( $\mathrm{HbA1c}$ ) levels in subjects with type 2 diabetes and chronic periodontitis. Contemporary Clinical Trials 2013;36(2):515-26.

Engebretson S, Michaelowicz B, Seaquist ER, Reddy M, Lewis CE, Oates T, et al. The Diabetes and Periodontal Therapy Trial (DPTT). Journal of Dental Research 2012;91(Suppl A (AADR 41st Annual Meeting; Tampa, Florida)):Abstract No 568.

* Engebretson SP, Hyman LG, Michalowicz BS, Schoenfeld ER, Gelato MC, Hou W, et al. The effect of nonsurgical periodontal therapy on hemoglobin Alc levels in persons with type 2 diabetes and chronic periodontitis: a randomized clinical trial. JAMA 2013;310(23):2523-32.

Michalowicz BS, Hyman L, Hou W, Oates TW Jr, Reddy M, Paquette DW, et al. Factors associated with the clinical response to nonsurgical periodontal therapy in people with type 2 diabetes mellitus. Journal of the American Dental Association 2014;145(12):1227-39.

\section{Gay 2014 \{published data only\}}

Gay IC, Tran DT, Cavender AC, Weltman R, Chang J, Luckenbach $\mathrm{E}$, et al. The effect of periodontal therapy on glycaemic control in a Hispanic population with type 2 diabetes: a randomized controlled trial. Journal of Clinical Periodontology 2014;41(7):673-80.

\section{Gilowski 2012 \{published and unpublished data\}}

Gilowski L, Kondzielnik P, Wiench R, Plocica I, Strojek K, Krzeminski TF. Efficacy of short-term adjunctive subantimicrobial dose doxycycline in diabetic patients-randomized study. Oral Diseases 2012; Vol. 18, issue 8:763-70.

\section{Grossi 1997 \{published and unpublished data\}}

* Grossi SG, Skrepinski FB, DeCaro T, Robertson DC, Ho AW, Dunford RG, et al. Treatment of periodontal disease in diabetics reduces glycated hemoglobin. Journal of Periodontology 1997;68(8):713-9.

Skrepinski F, Grossi S, Ho A, Dunford R, Genco RJ, DeCaro T, et al. A model of treatment of periodontal disease in native Americans with NIDDM. Journal of Periodontology 1996;67:71-2.

Haerian Ardakani 2014 \{published data only\}

Haerian Ardakani A, Asadi Y, Afkhami Ardakani M, Nooorelahi M, Sooratgar A. Effect of topical tetracycline gel with non surgical periodontal therapy on $\mathrm{Hba} 1 \mathrm{C}$ and lipid profile in type 2 diabetic patients: a clinico-biochemical study. Journal of Shahid Sadoughi University of Medical Sciences 2014;22(4):1387-95.

\section{Jones 2007 \{published and unpublished data\}}

Jones JA, Miller DR, Wehler CJ, Rich S, Krall E, Christiansen CL, et al. Study design, recruitment, and baseline characteristics: the Department of Veterans Affairs Dental Diabetes Study. Journal of Clinical Periodontology 2007;34(1):40-5. [PUBMED: 17040483]

* Jones JA, Miller DR, Wehler CJ, Rich SE, Krall-Kaye EA, McCoy LC, et al. Does periodontal care improve glycemic control? The Department of Veterans Affairs Dental Diabetes Study. Journal of Clinical Periodontology 2007;34(1):46-52.

McCoy LC, Wehler CJ, Rich SE, Garcia RI, Miller DR, Jones JA. Adverse events associated with chlorhexidine use: results from the Department of Veterans Affairs Dental Diabetes Study. Journal of the American Dental Association 2008;139(2):178-83. [PUBMED: 18245686]

\section{Katagiri 2009 \{published and unpublished data\}}

Katagiri S, Nitta H, Nagasawa T, Uchimura I, Izumiyama H, Inagaki $\mathrm{K}$, et al. Multi-center intervention study on glycohemoglobin (HbA1c) and serum, high-sensitivity CRP (hsCRP) after local anti-infectious periodontal treatment in type 2 diabetic patients with periodontal disease. Diabetes Research and Clinical Practice 2009;83(3):308-15.

\section{Kiran 2005 \{published and unpublished data\}}

Kiran M, Arpak N, Unsal E, Erdogan MF. The effect of improved periodontal health on metabolic control in type 2 diabetes mellitus. Journal of Clinical Periodontology 2005;32(3):266-72. 
Koromantzos 2011 \{published and unpublished data\}

* Koromantzos PA, Makrilakis K, Dereka X, Katsilambros N, Vrotsos IA, Madianos PN. A randomized, controlled trial on the effect of non-surgical periodontal therapy in patients with type 2 diabetes. Part I: effect on periodontal status and glycaemic control. Journal of Clinical Periodontology 2011;38(2):142-7.

Koromantzos PA, Makrilakis K, Dereka X, Offenbacher S, Katsilambros N, Vrotsos IA, et al. Effect of non-surgical periodontal therapy on C-reactive protein, oxidative stress, and matrix metalloproteinase (MMP)- 9 and MMP-2 levels in patients with type 2 diabetes: a randomized controlled study. Journal of Periodontology 2012; Vol. 83, issue 1:3-10.

\section{Kothiwale 2013 \{published and unpublished data\}}

Kothiwale SV, Kothiwale VA, Bhargava PV. Effect of noninvasive periodontal therapy on glycaemic control in type 2 diabetes mellitus patients - a randomized control trial. Diabetes 2013;62(Suppl 1):Abstract No A229.

Li 2011 \{published data only\}

Li Z, Sha YQ, Zhang BX, Zhu L, Kang J. [Effect of community periodontal care intervention on periodontal health and glycemic control in type 2 diabetic patients with chronic periodontitis]. [Chinese]. Beijing da Xue Xue Bao (Yi Xue Ban/ Journal of Peking University. Health Sciences) 2011;43(2):285-9.

\section{Llambés 2008 \{published and unpublished data\}}

Llambés F, Silvestre FJ, Hernandez-Mijares A, Guiha R, Caffesse R. Effect of non-surgical periodontal treatment with or without doxycycline on the periodontium of type 1 diabetic patients. Journal of Clinical Periodontology 2005;32(8):915-20. [PUBMED: 15998278]

* Llambés F, Silvestre FJ, Hernandez-Mijares A, Guiha R, Caffesse R. The effect of periodontal treatment on metabolic control of type 1 diabetes mellitus. Clinical Oral Investigations 2008;12(4):337-43.

\section{Macedo 2014 \{published data only\}}

Macedo G, Arthur B, Novaes J, Sergio L, Souza S, Mario T, et al. Additional effects of a PDT on nonsurgical periodontal treatment with doxycycline in type II diabetes: a randomized, controlled clinical trial. Lasers in Medical Science 2014;29:881-6. [DOI: 10.1007/s10103-013-1285-6]

\section{Madden 2008 \{published data only\}}

Madden TE, Herriges B, Boyd LD, Laughlin G, Chiodo G, Rosenstein D. Alterations in $\mathrm{HbAlc}$ following minimal or enhanced non-surgical, non-antibiotic treatment of gingivitis or mild periodontitis in type 2 diabetic patients: a pilot trial. The Journal of Contemporary Dental Practice 2008;9(5):9-16.

\section{Miranda 2014 \{published and unpublished data\}}

Miranda TS, Feres M, Perez-Chaparro PJ, Faveri M, Figueiredo LC, Tamashiro NS, et al. Metronidazole and amoxicillin as adjuncts to scaling and root planing for the treatment of type 2 diabetic subjects with periodontitis: 1-year outcomes of a randomized placebo-controlled clinical trial. Journal of Clinical Periodontology 2014;41(9):890-9.

\section{Moeintaghavi 2012 \{published data only\}}

Moeintaghavi A, Arab HR, Bozorgnia Y, Kianoush K, Alizadeh M. Non-surgical periodontal therapy affects metabolic control in diabetics: a randomized controlled clinical trial. Australian Dental Journal 2012; Vol. 57, issue 1:31-7.

NCT00801164 \{published and unpublished data\}

NCT00801164. Exploratory study of iocide oral rinse in a diabetic population (diabetes). clinicaltrials.gov/show/ NCT00801164 (2012).

\section{O'Connell 2008 \{published data only\}}

O'Connell PA, Taba M, Nomizo A, Foss Freitas MC, Suaid FA, Uyemura SA, et al. Effects of periodontal therapy on glycemic control and inflammatory markers. Journal of Periodontology 2008;79(5):774-83.

\section{Pradeep 2013 \{published data only\}}

Pradeep AR, Rao NS, Bajaj P, Kumari M. Efficacy of subgingivally delivered simvastatin in the treatment of patients with type 2 diabetes and chronic periodontitis: A randomized doublemasked controlled clinical trial. Journal of Periodontology 2013;84(1):24-31.

\section{Raman 2014 \{published data only\}}

Raman RP, Taiyeb-Ali TB, Chan SP, Chinna K, Vaithilingam RD. Effect of nonsurgical periodontal therapy versus oral hygiene instructions on Type 2 diabetes subjects with chronic periodontitis: a randomised clinical trial. BMC Oral Health 2014;14(1):2-19.

\section{Rocha 2001 \{published data only\}}

Rocha M, Nava LE, Vazquez de la Torre C, Sanchez-Marin F, Garay-Sevilla ME, Malacara JM. Clinical and radiological improvement of periodontal disease in patients with type 2 diabetes mellitus treated with alendronate: a randomized, placebo-controlled trial. Journal of Periodontology 2001;72(2):204-9

Rodrigues 2003 \{published and unpublished data\}

Rodrigues DC, Taba MJ, Novaes AB, Souza SL, Grisi MF. Effect of non-surgical periodontal therapy on glycemic control in patients with type 2 diabetes mellitus. Journal of Periodontology 2003;74(9):1361-7.

\section{Santos 2009 \{published and unpublished data\}}

Santos VR, Lima JA, De Mendonca AC, Maximo MBB, Faveri M, Duarte PM. Effectiveness of full-mouth and partial-mouth scaling and root planing in treating chronic periodontitis in subjects with type 2 diabetes. Journal of Periodontology 2009;80:1237-53

\section{Santos 2012 \{published and unpublished data\}}

Santos VR, Ribeiro FV, Lima JA, Miranda TS, Feres M, Bastos MF, et al. Partial- and full-mouth scaling and root planing in type 2 diabetic subjects: a 12-month follow-up of clinical parameters and levels of cytokines and osteoclastogenesis-related factors. Journal of Periodontal Research 2012;47(1):45-54. 


\section{Santos 2013 \{published data only\}}

Santos VR, Lima JA, Miranda TS, Gonçalves TE, Figueiredo LC, Faveri M. Full-mouth disinfection as a therapeutic protocol for type-2 diabetic subjects with chronic periodontitis: Twelvemonth clinical outcomes. A randomized controlled clinical trial. Journal of Clinical Periodontology 2013;40(2):155-62.

\section{Singh 2008 \{published data only\}}

Singh S, Kumar V, Kumar S, Subbappa A. The effect of periodontal therapy on the improvement of glycaemic control in patients with type 2 diabetes mellitus: A randomized controlled clinical trial. International Journal of Diabetes in Developing Countries 2008;28(2):38-44.

\section{Skaleric 2004 \{published and unpublished data\}}

Skaleric U, Schara R, Medvescek M, Hanlon A, Doherty F, Lessem J. Periodontal treatment by Arestin and its effects on glycemic control in type 1 diabetes patients. Journal of the International Academy of Periodontology 2004; Vol. 6:160-5.

\section{Sun 2011 \{published data only\}}

Sun WL, Chen LL, Zhang SZ, Wu YM, Ren YZ, Qin GM. Inflammatory cytokines, adiponectin, insulin resistance and metabolic control after periodontal intervention in patients with type 2 diabetes and chronic periodontitis. Internal Medicine 2011; Vol. 50, issue 15:1569-74.

\section{Tsalikis 2014 \{published data only\}}

Tsalikis L, Sakellari D, Dagalis P, Boura P, Konstantinidis A. Effects of doxycycline on clinical, microbiological and immunological parameters in well-controlled diabetes type-2 patients with periodontal disease: a randomized, controlled clinical trial. Journal of Clinical Periodontology 2014;41(10):972-80.

\section{Yun 2007 \{published and unpublished data\}}

Yun F, Firkova El, Jun-Qi L, Xun H. Effect of non-surgical periodontal therapy on patients with type 2 diabetes mellitus. Folia Medica 2007;49(1-2):32-6.

\section{Zhang 2013 \{published data only\}}

Zhang H, Li C, Shang S, Luo Z. Scaling and root planing with enhanced root planing on healthcare for type 2 diabetes mellitus: A randomized controlled clinical trial. Journal of Dental Sciences 2013;8(3):272-80.

\section{References to studies excluded from this review}

\section{Albrecht 1988 \{published data only\}}

Albrecht M, Banoczy J, Gyenes V, Ember G, Rigo O, Valkovics M, et al. Treatment of gingivitis and periodontal diseases with insadol in diabetics. Fogorvosi Szemle 1988;81:65-71.

\section{Al-Mubarak 2002 \{published data only\}}

Al-Mubarak S, Ciancio S, Aljada A, Mohanty P, Ross C, Dandona P. Comparative evaluation of adjunctive oral irrigation in diabetics. Journal of Clinical Periodontology 2002;29(4):295-300.

\section{Cinar 2014 \{published data only\}}

Cinar AB, Schou L. Impact of empowerment on toothbrushing and diabetes management. Oral Health \& Preventive Dentistry 2014;12(4):337-44.

\section{Gorbacheva 2010 \{published data only\}}

Gorbacheva IA, Orekhova LY, Shestakova LA, Musayeva RS, Silina AS. Alternative pathogenetic approach to the treatment of periodontal inflammatory diseases in diabetic patients with polyorganic disorders. Azerbaidzhanskii Meditsinskii Zhurnal (Azerbaijan Medical Journal) 2010;(3):81-7.

\section{Hagiwara 2002 \{published and unpublished data\}}

Hagiwara S, Ogasawara Y, Tanaka A. Effect of non-surgical periodontal therapy on diabetic metabolic control. Journal of Dental Research 2002;81(Suppl 1):Abstract No 1551.

\section{Khader 2010 \{published data only\}}

Khader YS, Al Habashneh R, Al Malalheh M, Bataineh A. The effect of full-mouth tooth extraction on glycemic control among patients with type 2 diabetes requiring extraction of all remaining teeth: a randomized clinical trial. Journal of Periodontal Research 2010; Vol. 45, issue 6:741-7.

\section{Llambés 2012 \{published data only\}}

Llambés F, Silvestre FJ, Hernández-Mijares A, Guiha R, Bautista D, Caffesse R. Efect of periodontal disease and non surgical periodontal treatment on C-reactive protein. Evaluation of type 1 diabetic patients. Medicina Oral, Patología Oral y Cirugía Bucal 2012;17(4):e562-8.

\section{Mansouri 2006 \{published and unpublished data\}}

Mansouri SS, Esteghamati A, Yousefi Y. Evaluation of first phase non-surgical periodontal therapy on diabetes control. Iranian Journal of Diabetes and Lipid Disorders 2006;6:E13.

\section{Munenaga 2013 \{published data only\}}

Munenaga Y, Hiroshima Study G, Yamashina T, Tanaka J, Nishimura F. Improvement of glycated hemoglobin in Japanese subjects with type 2 diabetes by resolution of periodontal inflammation using adjunct topical antibiotics: Results from the Hiroshima Study. Diabetes Research and Clinical Practice 2013;100(1):53-60.

\section{NCT01255254 \{published data only\}}

NCT01255254. The effect of oral hygiene and full mouth scaling on metabolic control in patients with Type II diabetes. clinicaltrials.gov/show/NCT01255254 (2010).

\section{Promsudthi 2005 \{published data only\}}

Promsudthi A, Pimapansri S, Deerochanawong C, Kanchanavasita W. The effect of periodontal therapy on uncontrolled type 2 diabetes mellitus in older subjects. Oral Diseases 2005;11(5):293-8.

\section{Taylor 2011 \{published data only\}}

Taylor GW, Borgnakke WS. Relationship of periodontal infection to diabetes: glycemic control, complications and occurrence. Journal of Dental Health (Japanese Society for Oral Health) 2011;61(Suppl):170-7. 


\section{References to studies awaiting assessment}

Al-Mubarak 2010 \{published data only\}

Al Mubarak S, Abou Rass M, Alsuwyed A, Al-Zoman K, Al Sohail A, Sobki $S$, et al. A new paradigm between mechanical scaling and root planing combined with adjunctive chemotherapy for glycated hemoglobin improvement in diabetics. International Journal of Diabetes Mellitus 2010;2(3):158-64.

Botero 2013 \{published data only\}

* Botero JE, Yepes FL, Ochoa SP, Hincapie JP, Roldan N, Ospina CA, et al. Effects of periodontal non-surgical therapy plus azithromycin on glycemic control in patients with diabetes: a randomized clinical trial. Journal of Periodontal Research 2013;48(6):706-12.

Hincapié JP, Castrillón CA, Yepes FL, Roldan N, Becerra MA, Moreno SM, et al. Microbiological effects of periodontal therapy plus azithromycin in patients with diabetes: results from a randomized clinical trial. Acta Odontológica Latinoamericana 2014;27(2):89-95.

Chee 2006 \{published data only\}

* Chee HK, Lim LP, Tay F, Thai AC, Sum CF. Non-surgical periodontal therapy and serum lipid levels in patients with diabetes mellitus. Annals of the Royal Australasian College of Dental Surgeons 2006;18:46.

Chee HK, Lim LP, Tay F, Thai AC, Sum CF. Non-surgical periodontal treatment and lipid levels in diabetic patients. Annals of the Royal Australasian College of Dental Surgeons 2008;19:183.

Lin 2012 \{published and unpublished data\}

Lin SJ, Tu YK, Tsai SC, Lai SM, Lu HK. Non-surgical periodontal therapy with and without subgingival minocycline administration in patients with poorly controlled type II diabetes: a randomized controlled clinical trial. Clinical Oral Investigations 2012;16(2):599-609.

\section{Nassar 2014 \{published data only\}}

Nassar PO, Poleto R, Salvador CS, Felipetti FA, Nassar CA. Onestage full-mouth disinfection and basic periodontal treatment in patients with diabetes mellitus. Journal of Public Health 2014;22(1):81-6.

\section{References to ongoing studies}

ACTRN12605000260628 \{published and unpublished data\} ACTRN12605000260628. Assessment of diabetes after periodontal treatment. www.anzctr.org.au/Trial/Registration/ TrialReview.aspx?ACTRN=12605000260628 (2005).

ACTRN12605000340639 \{published and unpublished data\} ACTRN12605000340639. RMI adult oral health programme. www.anzctr.org.au/Trial/Registration/TrialReview.aspx? ACTRN $=12605000340639$ (2005).
IRCT2014082417587N7 \{published data only\}

IRCT2014082417587N7. The effect of non-surgical periodontal therapy plus doxycycline on $\mathrm{HbA1c}$ in patients with type 2 diabetes mellitus (DM). www.irct.ir/searchresult.php? keyword=\&id=17587\&number=7\&prt=7287\&total $=10 \& m=1$ (2014).

ISRCTN15334496 \{published and unpublished data\}

Vergnes JN, Arrivé E, Gourdy P, Hanaire H, Rigalleau V, Gin H, et al. Periodontal treatment to improve glycaemic control in diabetic patients: study protocol of the randomized, controlled DIAPERIO trial. Trials 2009;10:65.

ISRCTN83229304 \{published and unpublished data\}

ISRCTN83229304. Periodontitis and type 2 diabetes mellitus. www.isrctn.com/ISRCTN83229304 (2010).

NCT00016835 \{published data only\}

NCT00016835. Treating periodontal infection: effects on glycemic control. clinicaltrials.gov/show/NCT00016835 (2001).

NCT01291875 \{published data only\}

NCT01291875. Periodontal treatment and metabolic control in Type 2 diabetic patients. clinicaltrials.gov/ct2/show/ NCT01291875 (2011).

NCT01881074 \{published data only\}

NCT01881074. The influence of periodontal treatment on gingival inflammatory response of the Type II diabetic patient. clinicaltrials.gov/ct2/show/NCT01881074 (2012).

NCT01901926 \{published data only\}

NCT01901926. Impact of non surgical periodontal treatment on glycemic control in Type II diabetics. clinicaltrials.gov/ct2/show/ NCT01901926 (2012).

NCT01904422 \{published data only\}

NCT01904422. Evaluation of the effectiveness of intensive periodontal treatment as compared to conventional periodontal treatment on the level of glycosylated hemoglobin in patients with decompensated type 2 diabetes mellitus: randomized clinical trial. clinicaltrials.gov/ct2/show/ NCT01904422 (2013).

NCT01964833 \{published and unpublished data\} NCT01964833. Combination of photodynamic therapy and periodontal treatment in patients with type 2 diabetes mellitus: randomized, double-blind clinical trial. clinicaltrials.gov/ct2/ show/NCT01964833 (2013).

U1111-1124-3635 \{published data only\}

U1111-1124-3635. Influence of periodontal treatment in periodontitis and diabetes control. www.ensaiosclinicos.gov.br/ rg/RBR-8dfrpt/ (2012).

\section{Additional references}

\section{Alam 2009}

Alam R, Sturt J, Lall R, Winkley K. An updated meta-analysis to assess the effectiveness of psychological interventions 
delivered by psychological specialists and generalist clinicians on glycaemic control and on psychological status. Patient Education and Counseling 2009;75(1):25-36.

\section{Armitage 1999}

Armitage GC. Development of a classification system for periodontal diseases and conditions. Annals of Periodontology 1999;4(1):1-6.

\section{Begg 1994}

Begg CB, Mazumdar M. Operating characteristics of a rank correlation test for publication bias. Biometrics 1994;50(4):1088-101.

\section{Berlin 1997}

Berlin JA. Does blinding of readers affect the results of metaanalyses? University of Pennsylvania Meta-analysis Blinding Study Group. Lancet 1997;350(9072):185-6.

\section{Bonito 2004}

Bonito AJ, Lohr KN, Lux L, Sutton S, Jackman A, Whitener L, et al. Effectiveness of antimicrobial adjuncts to scaling and root-planing therapy for periodontitis. United States Agency for Healthcare Research and Quality: Evidence Report/Technology Assessment (Summary) 2004;88:1-4.

\section{Borgnakke 2014}

Borgnakke WS, Chapple ILC, Genco RJ, Armitage G, Bartold PM, D'Aiuto F, et al. The multi-center randomized controlled trial (RCT) published by the Journal of the American Medical Association (JAMA) on the effect of periodontal therapy on glycated hemoglobin (HbA1c) has fundamental problems. Journal of Evidence-Based Dental Practice 2014;14(3):127-32.

\section{Buchwald 2013}

Buchwald S, Kocher T, Biffar R, Harb A, Holtfreter B, Meisel P. Tooth loss and periodontitis by socio-economic status and inflammation in a longitudinal population-based study. Journal of Clinical Periodontology 2013;40(3):203-11.

\section{Bunn 1981}

Bunn HF. Evaluation of glycosylated hemoglobin diabetic patients. Diabetes 1981;30(7):613-7.

\section{Chew 2015}

Chew BH, Sherina MS, Hassan NH. Association of diabetesrelated distress, depression, medication adherence, and healthrelated quality of life with glycated hemoglobin, blood pressure, and lipids in adult patients with type 2 diabetes: a crosssectional study. Therapeutics and Clinical Risk Management 2015;11:669-81.

\section{Darré 2008}

Darré L, Vergnes JN, Gourdy P, Sixou M. Efficacy of periodontal treatment on glycaemic control in diabetic patients: A metaanalysis of interventional studies. Diabetes \& Metabolism 2008;34(5):497-506.

\section{DCCT 1993}

Diabetes Control and Complications Trial Research Group (DCCT). The effect of intensive treatment of diabetes on the development and progression of long-term complications in insulin-dependent diabetes mellitus. The New England Journal of Medicine 1993;329(14):977-86.

\section{Egger 1997}

Egger M, Davey-Smith G, Schneider M, Minder C. Bias in meta-analysis detected by a simple, graphical test. BMJ 1997;315(7109):629-34.

\section{Engebretson 2013a}

Engebretson S, Kocher T. Evidence that periodontal treatment improves diabetes outcomes: a systematic review and metaanalysis. Journal of Clinical Periodontology 2013;40(Suppl 14):S153-63.

\section{Firatli 1997}

Firatli E. The relationship between clinical periodontal status and insulin dependent diabetes mellitus. Results after 5 years. Journal of Periodontology 1997;68(2):136-40.

\section{Florkowski 2003}

Florkowski C. HbA1c standardisation issues: should New Zealand follow the DCCT or the IFCC position?. The New Zealand Medical Journal 2003;116(1171):U395.

\section{Franco 2012}

Franco RS. Measurement of red cell lifespan and aging. Transfusion Medicine and Hemotherapy 2012;39(5):302-7.

\section{Goldstein 2004}

Goldstein DE, Little RR, Lorenz RA, Malone JI, Nathan D, Peterson CM, et al. Tests of glycaemia in diabetes. Diabetes Care 2004;27(7):1761-73.

\section{GRADEpro [Computer program]}

McMaster University. GRADEpro. Version 3.6.1. McMaster University, 2014.

\section{Grossi 1998}

Grossi SG, Genco RJ. Periodontal disease and diabetes mellitus: a two-way relationship. Annals of Periodontology 1998;3(1):51-61.

\section{Hanas 2010}

Hanas R, John G, International HBA1c Consensus Committee. 2010 consensus statement on the worldwide standardization of the hemoglobin A1C measurement. Diabetes Care 2010;33(8):1903-4.

\section{Hex 2012}

Hex N, Bartlett C, Wright D, Taylor M, Varley D. Estimating the current and future costs of type 1 and type 2 diabetes in the UK, including direct health costs and indirect societal and productivity costs. Diabetic Medicine 2012;29(7):855-62.

\section{Higgins 2011}

Higgins JPT, Green S (editors). Cochrane Handbook for Systematic Reviews of Interventions Version 5.1.0 [updated March 2011]. The Cochrane Collaboration, 2011. Available from www.cochrane-handbook.org. 


\section{International Diabetes Federation 2013}

International Diabetes Federation. IDF Diabetes Atlas. 6th Edition. Brussels, Belgium: International Diabetes Federation, 2013. [ISBN 2-930229-85-3]

\section{Khaw 2001}

Khaw KT, Wareham N, Luben R, Bingham S, Oakes S, Welch A, et al. Glycated haemoglobin, diabetes, and mortality in men in Norfolk cohort of european prospective investigation of cancer and nutrition (EPIC-Norfolk). BMJ 2001;322(7277):15-8.

\section{Kornman 2014}

Kornman KS. Commentary: Periodontitis severity and progression are modified by various host and environmental factors. Journal of Periodontology 2014;85(12):1642-5.

\section{Kowall 2015}

Kowall B, Holtfreter B, Völzke H, Scipfe S, Mundt T, Rathmann W, et al. Pre-diabetes and well-controlled are not associated with periodontal disease: the SHIP Trend Study. Journal of Clinical Periodontology 2015;42(5):422-30.

\section{McCambridge 2014}

McCambridge J, Witton J, Elbourne DR. Systematic review of the Hawthorne effect: new concepts are needed to study research participation effects. Journal of Clinical Epidemiology 2014;67(3):267-77.

\section{Milward 2003}

Milward MR, Chapple IL. Classification of periodontal diseases: Where were we? Where are we now? Where are we going?. Dental Update 2003;30(1):37-44.

\section{Page 1998}

Page RC. The pathobiology of periodontal diseases may affect systemic diseases: inversion of a paradigm. Annals of Periodontology 1998;3(1):108-20.

\section{Papapanou 1996}

Papapanou PN. Periodontal diseases: epidemiology. Annals of Periodontology 1996;1(1):1-36.

\section{Peter 2007}

Peter S. Essentials Of Preventive and Community Dentistry. 3rd Edition. New Delhi: Arya (Medi) Publishing House, 2007.

\section{Preshaw 2012}

Preshaw PM, Alba AL, Herrera D, Jepsen S, Konstantinidis A, Makrilakis $\mathrm{K}$, et al. Periodontitis and diabetes: a two-way relationship. Diabetologia 2012;55(1):21-31.

\section{Rabi 2006}

Rabi DM, Edwards AL, Southern DA, Svenson LW, Sargious PM, Norton P, et al. Association of socio-economic status with diabetes prevalence and utilization of diabetes care services. BMC Health Services Research 2006;3(6):124.

\section{RevMan 2014 [Computer program]}

Copenhagen: The Nordic Cochrane Centre, The Cochrane Collaboration. Review Manager (RevMan). Version 5.3.
Copenhagen: The Nordic Cochrane Centre, The Cochrane Collaboration, 2014.

\section{Sandberg 2000}

Sandberg GE, Sundberg HE, Fjellstrom CA, Wikblad KF. Type 2 diabetes and oral health: a comparison between diabetic and non-diabetic subjects. Diabetes Reseach and Clinical Practice 2000;50(1):27-34.

\section{Seppälä 1993}

Seppälä B, Seppälä M, Ainamo J. A longitudinal study on insulin-dependent diabetes mellitus and periodontal disease. Journal of Clinical Periodontology 1993;20(3):161-5.

\section{Seuring 2015}

Seuring T, Archangelidi O, Suhrcke M. The economic costs of type 2 diabetes: a global systematic review. PharmacoEconomics 2015;33(8):811-31.

\section{Sgolastra 2013}

Sgolastra F, Severino M, Pietropaoli D, Gatto R, Monaco A. Effectiveness of periodontal treatment to improve metabolic control in patients with chronic periodontitis and type 2 diabetes: a meta-analysis of randomized clinical trials. Journal of Periodontology 2013;84(7):958-73.

\section{Stewart 2001}

Stewart JE, Wager KA, Friedlander AH, Zadeh HH. The effect of periodontal treatment on glycaemic control in patients with type 2 diabetes mellitus. Journal of Clinical Periodontolgy 2001;28(4):306-10.

\section{Stratton 2000}

Stratton IM, Adler AI, Neil HA, Mathews DR, Manley SE, Cull CA, et al. Association of glycaemia with macrovascular and microvascular complications of type 2 diabetes (UKPDS 35): prospective observational study. BMJ 2000;321(7258):405-12.

\section{Taylor 2001}

Taylor GW. Bidirectional interrelationships between diabetes and periodontal diseases: an epidemiological perspective. Annals of Periodontology 2001;6(1):99-112.

\section{Teeuw 2010}

Teeuw WJ, Gerdes VEA, Loos BG. Effect of periodontal treatment on glycemic control of diabetic patients. Diabetes Care 2010;33(2):421-7.

\section{UKPDS 1998}

United Kingdom Prospective Diabetes Study 33 (UKPDS). Intensive blood-glucose control with sulphonylureas or insulin compared with conventional treatment and risk of complications in patients with type 2 diabetes. Lancet 1998;352(9131):837-53.

\section{WHO 2013}

World Health Organization. Global action plan for the prevention and control of noncommunicable diseases (NCDs) 2013-2020. www.who.int/nmh/events/ncd_action_plan/en/. 


\section{WHO 2014}

World Health Organization. Global status report on noncommunicable diseases 2014. www.who.int/nmh/ publications/ncd-status-report-2014/en/.

\section{WHO 2015}

World Health Organization. Diabetes: Fact sheet № 312. www.who.int/mediacentre/factsheets/fs312/en/ (January 2015).

\section{Worthington 2015}

Worthington $\mathrm{H}$, Clarkson J, Weldon J. Priority oral health research identification for clinical decision-making. Evidencebased Dentistry 2015;16(3):69-71.

\section{CHARACTERISTICS OF STUDIES}

Characteristics of included studies [ordered by study ID]

\section{References to other published versions of this review}

\section{Simpson 2004}

Simpson T, Needleman I, Wild SH, Moles DR, Mills EJ. Treatment of periodontal disease for glycaemic control in people with diabetes. Cochrane Database of Systematic Reviews 2004, Issue 2. [DOI: 10.1002/14651858.CD004714]

\section{Simpson 2010}

Simpson TC, Needleman I, Wild SH, Moles DR, Mills EJ. Treatment of periodontal disease for glycaemic control in people with diabetes. Cochrane Database of Systematic Reviews 2010, Issue 5. [DOI: 10.1002/14651858.CD004714.pub2]

* Indicates the major publication for the study

Al-Zahrani 2009

Trial design: 3-arm, single-centre, parallel-design RCT
Location: Saudi Arabia
Setting: Hospital
Number of centres: 1 , King Abdulaziz University Hospital
Recruitment period: January to November 2008
Funding source: Supported by a grant from King Abdulaziz University

Participants

Inclusion criteria: Age $>35$ years, confirmed diagnosis of type 2 diabetes, generalized moderate to severe chronic periodontitis, and $\geq 20$ remaining teeth. The presence of clinical attachment loss $\geq 3 \mathrm{~mm}$ at $\geq 30 \%$ of sites was used to define generalized moderate to severe chronic periodontitis

Exclusion criteria: Pregnant women, patients with major diabetic complications, and patients who received periodontal treatment or antibiotic therapy 6 months before the study

Age at baseline: Overall: mean 52.21 yrs (SD 8.35); Gp A: mean 53.14 yrs (SD 10.91); Gp B: mean 51.42 yrs (SD 6.24); Gp C: mean 51.92 yrs (SD 7.28) $(P=0.87)$

Sex (M:F): Overall: M17:F26; Gp A M7:F8; Gp B M4:F10; Gp C M6:F8 (P=0.58)

Tobacco use (never/ever): Overall: 33/10; Gp A: 9/6; Gp B: 13/1; Gp C: 11/3 ( $P=0.28)$

Alcohol consumption: Not reported

Diabetes type: All T2, as required by inclusion criterion

Duration since diabetes diagnosis: Not reported

Antidiabetic therapy: Insulin - Overall: $n=12$ (28\%); Gp A: $n=3(20 \%) ; G p ~ B: n=4(29 \%) ; G p ~ C: n=5$ $(36 \%)$

Oral hypoglycaemic medication - Quote: "About $72 \%$ and $28 \%$ of the participants were taking, respectively, oral hypoglycaemic medication and insulin to control their diabetes. None of the participants reported a change in the types or doses of their medications during the study period"

Metabolic control: Poor mean $\mathrm{HbA1c}$ at baseline 
Al-Zahrani 2009 (Continued)

Mean HbA1c at baseline: Overall: 8.80\% (SD 1.96); Gp A: 8.75\% (SD 1.43); Gp B: 8.42\% (SD 1.65); Gp C: 9.25\% (SD 2.71) $(P=0.56)$

Other medical conditions: Not reported

Other investigations: No additional investigations undertaken

Number randomised: 45

Number evaluated: 43 (2 lost to follow-up, allocated group not stated - possible to identify 1 each from Gps B+C - Gp A: $n=15 ; G p$ B: $n=14 ; G p ~ C: ~ n=14)$

Comparison: SRP + OHI versus SRP + OHI + doxycycline versus SRP + OHI + aPDT
Gp A $(\mathrm{n}=15): \mathrm{SRP}$ only $+\mathrm{OHI}$
$\mathrm{Gp} \mathrm{B}(\mathrm{n}=14): \mathrm{SRP}+\mathrm{OHI}+2 \times 100 \mathrm{mg}$ doxycycline, then $100 \mathrm{mg}$ daily for 13 days
$\mathrm{Gp} \mathrm{C}(\mathrm{n}=14): \mathrm{SRP}+\mathrm{OHI}+$ aPDT (using $0.01 \%$ methylene blue irrigator (as supplied in pre-filled syringe
by manufacturer), irradiated with $670 \mathrm{~nm}$ non-thermal diode laser)
All participants received individualised OHI at baseline, and SRP was performed across 4 sessions with-
in 7 days, using ultrasonic and manual instruments under local anaesthesia if necessary
Duration of follow-up: 3 months

Outcomes Primary: HbAlc, recorded at baseline and 3 months

Secondary: PI, BOP, CAL, and PPD, recorded at baseline and 3 months

Notes Sample size calculation: A total sample size of 42 subjects (14 in each group) was required to detect a difference of $1 \mathrm{~mm}$ between the highest and lowest means with $80 \%$ power and an assumed common standard deviation of 0.8 at a significance level of $P<0.05$. To compensate for any loss to follow-up, 45 patients were included

\section{Data analysis method: Assumed ITT}

HbA1c assessment method: Commercially available Dimension and Flex HA1C automated processor, Dade Behring, UK

Adverse events: Quote: "None of the patients reported any complications associated with the use of PDT therapy, such as burning sensations, discomfort, or pain"

SES: Education level attained reported

Overall: Illiterate $n=15$ (M:4; F:11); elementary $n=12$ (M:5; F:7); > elementary $n=16$ (M:8; F:8)

Gp A: Illiterate $n=5$; elementary $n=6$; >elementary $n=4$

Gp B: Illiterate $n=3$; elementary $n=4$; >elementary $n=7$

Gp C: Illiterate $n=7$; elementary $n=2$; >elementary $n=5$

Conflict of interests: Quote: "The authors report no conflicts of interest related to this study"

Trial ID: Not reported

\section{Risk of bias}

\begin{tabular}{lll}
\hline Bias & Authors' judgement & Support for judgement \\
\hline $\begin{array}{l}\text { Random sequence genera- } \\
\text { tion (selection bias) }\end{array}$ & Low risk & $\begin{array}{l}\text { A simple randomisation approach using computer-generated random num- } \\
\text { bers was employed to assign subjects to } 1 \text { of the following } 3 \text { treatment modal- } \\
\text { ities }\end{array}$ \\
\hline
\end{tabular}


Al-Zahrani 2009 (Continued)

Allocation concealment (selection bias)
Unclear risk

Quote: "Allocations of subjects to their assigned groups were kept with principal investigator (Al-Zahrani) in sealed envelopes, and were given to the coauthor who was performing the treatment immediately prior to the treatment schedule. The primary investigator was not involved in the immediate inclusion/exclusion of subjects and has no prior knowledge of the subjects' periodontal findings" (from correspondence with the principal author)

Comment: No indication if sealed envelopes were opaque

\begin{tabular}{|c|c|c|}
\hline Blinding of participants & High risk & Interventions different and no placebos used \\
\hline $\begin{array}{l}\text { Blinding of clinical opera- } \\
\text { tor }\end{array}$ & High risk & Not possible \\
\hline \multirow{2}{*}{$\begin{array}{l}\text { Incomplete outcome data } \\
\text { (attrition bias) } \\
\text { All outcomes }\end{array}$} & Unclear risk & $\begin{array}{l}\text { Quote: "Two patients were lost to follow-up; one travelled outside the country, } \\
\text { and the other one refused to return for follow-up" }\end{array}$ \\
\hline & & $\begin{array}{l}2 \text { of the original } 45 \text { randomised were lost to follow-up. Unlikely to introduce a } \\
\text { bias. Analysis assumed to have been ITT, but not specifically reported }\end{array}$ \\
\hline $\begin{array}{l}\text { Selective reporting (re- } \\
\text { porting bias) }\end{array}$ & Low risk & Planned outcomes reported in full \\
\hline Other bias & Low risk & No other apparent biases \\
\hline
\end{tabular}

Calbacho 2004

$\begin{array}{ll}\text { Methods } & \text { Trial design: } 2 \text {-arm, parallel-design RCT } \\ \text { Location: Chile } \\ \text { Setting: Primary care } \\ \text { Number of centres: Not reported } \\ \text { Recruitment period: Not reported } \\ \text { Funding source: Not reported }\end{array}$

Participants

Inclusion criteria: Aged 40-60, diagnosis of T2 DM with poor metabolic control of diabetes and moderate chronic marginal periodontitis diagnosis without treatment of this disease from 1 year or more

Exclusion criteria: Any other treatment or medication (except diabetes), less than 8 teeth (excluding third molars)

Age at baseline: Overall: mean 50.3 yrs (SD 6.2); Gp A: mean 52.8 yrs (SD 5.4); Gp B: mean 47.8 yrs (SD 6.1). No P value reported

Sex (M:F): Overall M10:F14; Gp A: M4:F8; Gp B: M6:F6. No P value reported

Tobacco use: All non-smokers

Alcohol consumption: Not reported

Diabetes type: All T2 DM

Duration since diabetes diagnosis: Both groups 10.0 yrs (SD 3.4)

Metabolic control: Poor mean $\mathrm{HbA1c}$ at baseline 
Antidiabetic therapy: All in receipt of oral hypoglycaemic medication only

HbA1c assessment method: High-performance liquid chromatography

Other clinical investigations: Mean blood glucose levels

Number randomised: 24

Number evaluated: 24

\begin{tabular}{|c|c|c|}
\hline Interventions & \multicolumn{2}{|c|}{$\begin{array}{l}\text { Comparison: SRP + doxycyline versus OHI } \\
\text { Gp A: }(n=12) \text { "conventional" periodontal treatment + doxycycline } 100 \text { mg daily for } 10 \text { days } \\
\text { Gp B: }(n=12) \text { OHI only }\end{array}$} \\
\hline Outcomes & \multicolumn{2}{|c|}{$\begin{array}{l}\text { Primary: } \mathrm{HbA} 1 \mathrm{c} \text {, at baseline, } 2 \text { and } 4 \text { months } \\
\text { Secondary: PPD, PI and BOP }\end{array}$} \\
\hline Notes & $\begin{array}{l}\text { Only abstract publish } \\
\text { pare report and exces } \\
\text { Author (Victor Calbacl } \\
\text { address is no longer v } \\
\text { SES: Not reported } \\
\text { Sample size calculat } \\
\text { Data analysis metho } \\
\text { HbA1c assessment m } \\
\text { Conflict of interests: } \\
\text { Adverse events: Not }\end{array}$ & $\begin{array}{l}\text { to date. Full study unpublished. Author states reason as "lack of time to pre- } \\
\text { of work in other areas" } \\
\text { id, and other authors have been non-responsive to email requests } \\
\text { n: Not reported } \\
\text { ITT } \\
\text { thod: High-performance liquid chromatography } \\
\text { ot reported } \\
\text { ported }\end{array}$ \\
\hline \multicolumn{3}{|l|}{ Risk of bias } \\
\hline Bias & Authors' judgement & Support for judgement \\
\hline $\begin{array}{l}\text { Random sequence genera- } \\
\text { tion (selection bias) }\end{array}$ & Low risk & $\begin{array}{l}\text { Block randomisation - method unexplained } \\
\text { Quote: "12 were at random assigned to a study group and the rest to a control } \\
\text { group" }\end{array}$ \\
\hline $\begin{array}{l}\text { Allocation concealment } \\
\text { (selection bias) }\end{array}$ & Unclear risk & Unknown \\
\hline Blinding of participants & High risk & Not possible \\
\hline $\begin{array}{l}\text { Blinding of clinical opera- } \\
\text { tor }\end{array}$ & High risk & Not possible \\
\hline $\begin{array}{l}\text { Incomplete outcome data } \\
\text { (attrition bias) } \\
\text { All outcomes }\end{array}$ & Low risk & All completed. ITT analysis \\
\hline
\end{tabular}


Calbacho 2004 (Continued)

Selective reporting (re- High risk Secondary data only reported as $\mathrm{P}$ values (no means or SDs provided despite porting bias) repeated email request). Also no detail of adverse events

Other bias

Unclear risk

Insufficient description in abstract and from author's comments to make a judgement. Full study unpublished other than as abstract, and therefore without peer-review although study lead confirms intention to publish

\section{Chen 2012}

Methods

Trial design: 3-arm, single-centre, parallel-design RCT

Location: Guangzhou

Setting: Not reported

Number of centres: 1

Recruitment period: November 2008 to October 2009

Funding source: 2 grants - both government sponsored: 1) Key Projects in the National Science and Technology Pillar Program (11 th 5-year plan periods), Beijing, China and 2) Technology Planning Project of Guangdong Province, China (grant 2010B031600117)

Participants

Inclusion criteria: Diagnosis T2 DM >1 year; no change in TP in the previous 2 months; no major diabetic complication (eg CHD); diagnosis of chronic periodontal disease (AAP criteria), $\geq 16$ teeth, $\geq 1 \mathrm{~mm}$ mean CAL; including mild, moderate and severe periodontitis

Exclusion criteria: Presence of systemic disease other than diabetes that could influence the course of periodontal disease; systemic antibiotic administration in last 3 months; pregnancy or lactation; refusal of written consent; active infections other than periodontitis; periodontal treatment in last 12 months

Age at baseline: Overall 60.3 yrs (SD 10.02); Gp A: mean 59.86 yrs (SD 9.48); Gp B: mean 57.91 yrs (SD 11.35); Gp C: mean 63.2 yrs (SD 8.51) $(P=0.052)$

Sex (M:F): Overall M66:F60; Gp A: M23;F19; Gp B: M26:F17; Gp C: M17:F24 (P=0.2)

Tobacco use: $\mathrm{Gp} \mathrm{A} \mathrm{n} \mathrm{=} \mathrm{7;} \mathrm{Gp} \mathrm{B} \mathrm{n} \mathrm{=} \mathrm{10;} \mathrm{Gp} \mathrm{C} n=7$ (former smoker: $G p A n=1 ; G p B n=1 ; G p C n=0)(P=$ 0.872)

Alcohol consumption: $G p A n=2 ; G p B n=4 ; G p C n=7(P=0.169)$

Diabetes type: All T2 DM

Duration since diabetes diagnosis: Gp A mean 8.69 yrs (SD 5.25); Gp B mean 6.93 yrs (SD 4.31); Gp C mean 9.56 yrs (SD 6.02) $(P=0.066)$

Metabolic control: Fair mean HbA1c at baseline

Mean HbA1c at baseline: Gp A 7.31\% (SD 1.23); Gp B 7.29\% (SD 1.55); Gp C 7.25\% (SD 1.49) (P > 0.05)

Antidiabetic therapy: All in receipt of oral hypoglycaemic medication (Gp A: 38; Gp B: 35; Gp C: 36), insulin (Gp A: 4; Gp B: 5; Gp C: 4), or diet (Gp A: 0; Gp B: 3; Gp C: 1) (P=0.574)

Other clinical investigations: Gingival recession, FPG (mmol/l), hsCRP (mg/L), TNF- $\alpha$ 9pg/ml), TC $(\mathrm{mmol} / \mathrm{l}), \mathrm{TG}(\mathrm{mmol} / \mathrm{l}), \mathrm{HDL}-\mathrm{C}(\mathrm{mmol} / \mathrm{l}), \mathrm{LDL}-\mathrm{C}(\mathrm{mmol} / \mathrm{l})$

Other medical conditions: None

Number randomised: 134 
Chen 2012 (Continued)

Number evaluated: 126 (loss to follow-up Gp A 3, Gp B 2, Gp C 3)

\section{Interventions Comparison: SRP + OHI (x 3) + subgingival debridement versus SRP + OHI $(x)$ 3) + supragingival prophylaxis versus no intervention}

Gp A ( $n=45)$ : SRP (at baseline; with local anaesthetic, no antibiotics or local antimicrobials, using standard Gracey curettes and ultrasonic instrumentation, and completed in $24 \mathrm{hrs})+\mathrm{OHI}(\times 3$ : at 1.5, 3 and 6 months check-ups) + subgingival debridement (at 3 months)

Gp B $(n=45)$ : SRP (at baseline; with local anaesthetic, no antibiotics or local antimicrobials, using standard Gracey curettes and ultrasonic instrumentation, and completed in $24 \mathrm{hrs})+\mathrm{OHI}(\mathrm{x}$ 3: at 1.5, 3 and 6 months check-ups) + supragingival prophylaxis (at 3 months; no intervention in deep periodontal pockets)

Gp C ( $n=44)$ : No intervention (delayed treatment until completion of study)

Duration of follow-up: 6 months with interim readings taken at 1.5 and 3 months

$\begin{array}{ll}\text { Outcomes } & \text { Primary: } \mathrm{HbAlc} \text { (at baseline, month 1.5, month } 3 \text { and month } 6 \text { ) } \\ & \text { Secondary: PI, BOP, mean PD, sites with PD }=4 \text { to } 5 \mathrm{~mm} \text {, sites with PD } \ddagger 6 \text { mm and mean CAL (all at } 1.5 \\ \text { months, } 3 \text { months and } 6 \text { months) }\end{array}$

$\begin{array}{ll}\text { Notes } & \begin{array}{l}\text { Sample size calculation: A priori calculation assuming SD of } 1 \% \text { at } 80 \% \text { power - approximately } 53 \text { per } \\ \text { group }\end{array}\end{array}$

Data analysis method: Per-protocol

HbA1c assessment method: Boronate-affinity chromatography

Conflict of interests: Authors report no conflict of interests

SES: Not reported

Adverse events: No adverse events reported by participants

\section{Risk of bias}

\begin{tabular}{lll}
\hline Bias & Authors' judgement & Support for judgement \\
\hline $\begin{array}{l}\text { Random sequence genera- } \\
\text { tion (selection bias) }\end{array}$ & Low risk & $\begin{array}{l}\text { Quote: "..computer-generated list of random numbers prepared by statisti- } \\
\text { cian" }\end{array}$ \\
\hline $\begin{array}{l}\text { Allocation concealment } \\
\text { (selection bias) }\end{array}$ & Unclear risk & $\begin{array}{l}\text { Quote: "Allocation concealed from researcher LC." Allocation overseen by "in- } \\
\text { dependent research nurse" }\end{array}$ \\
& $\begin{array}{l}\text { Sequentially numbered envelopes used 1-134 } \\
\text { Comment: No indication whether envelopes were opaque and sealed }\end{array}$ \\
\hline
\end{tabular}

\begin{tabular}{lll}
\hline Blinding of participants & High risk & Not possible due to difference in mechanical intervention \\
\hline $\begin{array}{l}\text { Blinding of clinical opera- } \\
\text { tor }\end{array}$ & Unclear risk & Not reported. DX undertook non-surgical periodontal treatment \\
\hline $\begin{array}{l}\text { Incomplete outcome data } \\
\text { (attrition bias) }\end{array}$ & High risk & $\begin{array}{l}\text { All patients accounted for with reasons provided. Per-protocol analysis: not all } \\
\text { participants analysed in groups randomised to, regardless of intervention ac- } \\
\text { tually received }\end{array}$ \\
\hline
\end{tabular}

Selective reporting (re- Low risk No evidence

porting bias) 
Chen 2012 (Continued)

Other bias Low risk No other apparent biases

Engebretson 2011

Methods

Trial design: 3-arm, single-centre, parallel-design RCT

Location: USA

Setting: Hospital

Number of centres: 1 , Naomi Berrie Diabetes Centre, Columbia University Medical Center

Recruitment period: Not reported

Funding source: Columbia University Office of Clinical Trials Pilot Award and National Institute of Dental and Craniofacial Research Award DE 00449 (SE). Collagenex Pharmaceutical Inc. provided subantimicrobial doxycycline doses (brand name: Periostat; Gp A) and placebos ( $\mathrm{Gps} B+\mathrm{C}$ ), and Columbia University Medical Center Research Pharmacy provided visually indistinguishable antimicrobial doxycyline doses (Gp B)

Participants

Inclusion criteria: Chronic periodontitis (CAL $>5 \mathrm{~mm}$ in at least 1 site in each quadrant). Duration since diagnosis $\geq 6$ months. Stable medication for diabetes for $\geq 3$ months (insulin or $\mathrm{OHA}$ )

Exclusion criteria: Present use of Coumadin (warfarin, anticoagulant); pregnancy or lack of birth control; chronic non-steroidal anti-inflammatory drug use or antibiotic use within 6 months; renal impairment, severe liver disease and grade 3 or 4 retinopathy

Age at baseline: Overall: mean $53.8 \mathrm{yrs}$ (SD 2.4); Gp A mean $53.2 \mathrm{yrs}$ (SD 3); Gp B mean $54.4 \mathrm{yrs}$ (SD 2); Gp C mean 53.8 yrs (SD 2) $(P=0.93)$

Sex (M:F): Overall: M20:F25; Gp A M7:F8; Gp B M7:F8; Gp C M6:F9 (P=0.91)

Tobacco use: Not reported

Alcohol consumption: Not reported

Diabetes type: Type 2 DM

Duration since diabetes diagnosis: Overall mean: 9 yrs; Gp A mean 11.6 yrs (SD 13.2); Gp B mean 6.1 yrs (SD 5.1); Gp C mean 7.6 yrs (SD 4.7) ( $P=0.33)$

Metabolic control: Fair mean HbA1c at baseline

Mean HbA1c at baseline: Gp A 7.9\% (SD 1.9); Gp B 7.6\% (SD 2.0); Gp C 8.2\% (SD 2.0) (P = 0.75)

Antidiabetic therapy: Inclusion criteria required stable dosage of oral hypoglycaemic agents or insulin. Study confirms use of medications remained constant during study period

Other clinical investigations: Plasma glucose $(\mathrm{mg} / \mathrm{dL})$, gingival recession

Number randomised: 45

Number evaluated: 34 (11 losses to follow-up)

Gp A ( $=15)$ : SRP (x 3: at baseline, 1 month and 3-month follow-up) + subantimicrobial doxycycline (20 $\mathrm{mg}$ twice a day, for 3 months duration: 2 vials at baseline - AM vial $14 \times 20 \mathrm{mg}$, PM vial $14 \times 20 \mathrm{mg}$; 1 vial at 2-week follow-up - $28 \times 20 \mathrm{mg} ; 1$ vial at 1 month follow-up - $120 \times 20 \mathrm{mg}$ ) 
Gp B ( $n=15)$ : SRP ( $x$ 3: at baseline, 1 month and 3-month follow-up) + antimicrobial dose doxycycline (100 mg daily, for 14 days: 2 vials at baseline - AM vial $14 \times 100 \mathrm{mg}$, PM vial 14 x placebo tablets; 1 vial at 2-week follow-up - 28 x placebo tablets; 1 vial at 1 month follow-up - $120 \times$ placebo tablets)

Gp C ( $n=15)$ : SRP (x 3: at baseline, 1 month and 3-month follow-up) + placebo (twice a day, for 3 months: 2 vials at baseline - AM vial 14 x placebo tablets, PM vial 14 x placebo tablets; 1 vial at 2-week follow-up - 28 x placebo tablets; 1 vial at 1 month follow-up - 120 x placebo tablets)

SRP for all groups including full mouth root planing, scaling with curettes and ultrasonic instrument, under local anaesthesia, lasting no longer than 2 hours

Duration of follow-up: 3 months

Outcomes Primary: HbAlc at baseline, 1 and 3 months (reported for baseline and 3 months: not 1 month)

Secondary: PPD, BOP, PI, CAL at baseline, 1 and 3 months (none reported)

Notes

Sample size calculation: No a priori calculations

Data analysis method: ITT analysis

SES: Not reported

Adverse events: "There were no serious adverse events reported during the study. Differences in adverse events between groups were not observed, and the treatments appeared to be well tolerated"

HbA1c assessment method: Automated affinity chromatography system (BioRad MicroMat II, Hercules, CA)

Conflict of interests: "The authors disclosed no conflicts of interest"

Author emailed to 2 separate addresses for further information/missing periodontal data, but no response received

\section{Risk of bias}

\begin{tabular}{|c|c|c|}
\hline Bias & Authors' judgement & Support for judgement \\
\hline $\begin{array}{l}\text { Random sequence genera- } \\
\text { tion (selection bias) }\end{array}$ & Low risk & "Assigned by computer generated table" to either group \\
\hline $\begin{array}{l}\text { Allocation concealment } \\
\text { (selection bias) }\end{array}$ & Low risk & $\begin{array}{l}\text { Comment: Matching and indistinguishable placebo and intervention were pro- } \\
\text { vided by the pharmacy in vials with unique codes }\end{array}$ \\
\hline Blinding of participants & Low risk & $\begin{array}{l}\text { Comment: Visually indistinguishable placebo given to control group. Time in- } \\
\text { tervals kept the same for each group }\end{array}$ \\
\hline $\begin{array}{l}\text { Blinding of clinical opera- } \\
\text { tor }\end{array}$ & Unclear risk & Comment: Study stated to be double-blinded, but not explained in text \\
\hline \multirow{2}{*}{$\begin{array}{l}\text { Incomplete outcome data } \\
\text { (attrition bias) } \\
\text { All outcomes }\end{array}$} & High risk & $\begin{array}{l}\text { Large number of withdrawals }(22 \%): 1 / 45 \text { withdrew: not indicated from which } \\
\text { arm; } 10 / 45 \text { lost to follow-up: Gp A: } n=6 ; G p \text { B: } n=1 ; G p ~ C: n=3 \text {. }\end{array}$ \\
\hline & & ITT analysis \\
\hline $\begin{array}{l}\text { Selective reporting (re- } \\
\text { porting bias) }\end{array}$ & High risk & No periodontal data reported despite being recorded \\
\hline Other bias & Low risk & No other apparent biases \\
\hline
\end{tabular}


Location: USA

Setting: Community

Number of centres: 5 - diabetes and dental clinics and communities associated with academic medical centres (deliberately selected for geographic diversity): -University of Alabama, Birmingham, Alabama;

-University of Minnesota and Hennepin County Medical Center, Minneapolis, Minnesota; -University of Texas Health Science Center, San Antonio, Texas;

-Stony Brook University, New York;

-University of Texas Health Science Center, Houston, Texas

Recruitment period: November 2009 - March 2012 (originally designed to run until May 2012) Enrollment stopped earlier than anticipated due to futility. Trial stopping rule based on power threshold of $40 \%$ demonstrating interim test statistic of $<-0.12$ t-test for $\mathrm{HbAlc}$ was -0.37 , consequently monitoring board recommended cessation of recruitment

Funding source: 2 x NIH/NIDCR grants: U01 DE018902 (awarded to S Engebretson); U01 DE018886 (awarded to L Hyman)

No detail re: provider/manufacturer of chlorhexidine mouthrinse to compare to conflict of interests declarations

Participants

Inclusion criteria: Either sex; aged 35 or more; with physician-diagnosed type 2 diabetes (duration of $>3$ months); an $\mathrm{HbA1c}$ value between $7-<9 \%$ at screening; under care of physician for management of diabetes; diagnosed with moderate-advanced chronic periodontitis (CAL/PD $>5 \mathrm{~mm}$ in 2 or $>$ quadrants); minimum of 16 natural teeth; received no periodontal treatment in prior 6 months; and agreed to continue current diabetes medications (unless medically indicated otherwise); and avoid pregnancy during the trial period

Exclusion criteria: Treatment required for extensive caries, abscess, or oral infection; limited life expectancy (<1 year); diabetes-related emergency in prior 30 days; NSAID use ( $>7$ days in prior 2 months. Except low-dose aspirin: $75-325 \mathrm{mg} / \mathrm{d}$ ); systemic immunosuppressant use; systemic antibiotic use ( $>6$ days during 30 days after enrolment); receiving dialysis; increased risk of bleeding complications; heavy alcohol consumption (mean $>2$ drinks/day for females and $>3$ drinks/day for males)

Age at baseline: Overall: mean 57.3 yrs (SD 10.1); Gp A: mean 56.7 yrs (SD 10.5); Gp B: mean 57.9 yrs (SD 9.6). No $P$ value reported

Sex (M:F): Overall: M277:F237; Gp A: M143:F114; Gp B: M134:F123. No P value reported

Tobacco use: Gp A: Never $n=129$; former $n=89$; current $n=39$

Gp B: Never $n=144$; former $n=86$; current $n=27$

Weight: Gp A: mean 99.5 kg (SD 24.3); Gp B: mean 97.5 kg (SD 21.7)

BMI: Gp A: 34.7 (SD 7.5); Gp B: 34.2 (SD 6.7)

Alcohol consumption: Not reported

Diabetes type: All T2 DM

Duration since diabetes diagnosis: Gp A: mean 12.3 yrs (SD 8.2); Gp B: 11.3 yrs (SD 8.4)

Metabolic control: Largely fair-poor mean $\mathrm{HbA1c}$ at baseline

Mean HbA1c at baseline: Overall: $<7.0 \% \mathrm{n}=22 ;>7.0 \%-<8.0 \% \mathrm{n}=297 ;>8.0 \%-<9.0 \% \mathrm{n}=179 ;>9.0 \%$ $<10.0 \% \mathrm{n}=16$

Gp A: $<7.0 \% n=12 ;>7.0 \%-<8.0 \% n=143 ;>8.0 \%-<9.0 \% n=93 ;>9.0 \%-<10.0 \% n=9$

Gp B: $<7.0 \% n=10 ;>7.0 \%-<8.0 \% n=154 ;>8.0 \%-<9.0 \% n=86 ;>9.0 \%-<10.0 \% n=7$ 
Antidiabetic therapy: All except 11 patients (2\% of 514 participants) were in receipt of oral hypoglycaemic medication, insulin, or combination treatment

Overall: No diabetes medications $n=11$; oral agents only $n=244$; insulin only $n=80$; combination of medications $\mathrm{n}=179$

Gp A: No diabetes medications $n=7$; oral agents only $n=117$; insulin only $n=40$; combination of medications $\mathrm{n}=93$

Gp B: No diabetes medications $n=4$; oral agents only $n=127$; insulin only $n=40$; combination of medications $n=86$

Other investigations: Change in insulin, fasting glucose levels, HOMA2 scores and diabetes medication from baseline; participants requiring periodontal/diabetes rescue therapy

\section{Other medical conditions:}

Overall: Angina $n=32$; myocardial infarction $n=43$; stroke $n=24$; hypertension $n=364$; kidney disease $\mathrm{n}=26$

Gp A: Angina $n=21$; myocardial infarction $n=22$; stroke $n=12$; hypertension $n=180$; kidney disease $n=$ 14

Gp B: Angina $n=11 ;$ myocardial infarction $n=21 ;$ stroke $n=12$; hypertension $n=184$; kidney disease $n$ $=12$

Number randomised: $514(\mathrm{Gp} \mathrm{A} n=257 ; \mathrm{Gp} \mathrm{B} n=257)$

\section{Number evaluated:}

ITT analysis (HbA1c outcome only):

Baseline: $\mathrm{Gp} \mathrm{An}=257 ; \mathrm{Gp} \mathrm{B} \mathrm{n}=257$

3 months: $\mathrm{Gp} \mathrm{An}=257 ; \mathrm{Gp} \mathrm{B} n=257$

6 months: $G p A n=257 ; G p B n=257$

Per-protocol analysis (all outcomes - all participants with HbA1c data at 6-month visit):

Baseline: $\mathrm{Gp} \mathrm{An}=240 ; \mathrm{Gp} \mathrm{B} \mathrm{n}=235$

3 months: Gp A $n=233 ; \mathrm{Gp} \mathrm{B} n=227$ (missed 3-month visit: Gp A $n=6 ; \mathrm{Gp} \mathrm{B} n=7$. Periodontal data missing: $G p A n=1 ; G p B n=1$ )

6 months: $G p A n=240 ; G p B n=233$ (periodontal data missing: $G p A n=0 ; G p B n=2$ )

Interventions

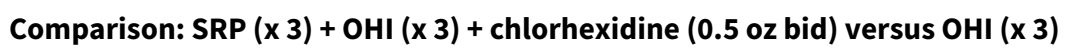

Gp A ( $n=257$ ): SRP (at baseline, 3 and 6 months: initial SRP >160 min treatment with local anaesthesia over 2 or more sessions, and completed within 42 days of initial baseline visit; SRP at 3 and 6 months comprised of a single 1 hour session each time) $+\mathrm{OHI}$ and provision of $0.12 \%$ chlorhexidine gluconate oral rinse ( $0.5 \mathrm{oz}$ twice daily for 2 weeks), toothbrush, toothpaste, and dental floss

$\mathrm{Gp} \mathrm{B} \mathrm{(} \mathrm{n}=257)$ : OHI at baseline, 3 months and 6 months (followed by offer of SRP after 6-month visit)

Duration of follow-up: 6 months

\section{Outcomes}

Primary: HbA1c (at baseline, 3 and 6 months)

Secondary: GI, BOP, PPD and CAL (at baseline, 3 and 6 months) ror). Accounting for attrition rate of $20 \%$, planned sample size was 600 (300 in each arm)

Data analysis: ITT (periodontal data provided per-protocol analysis; however, all periodontal parameters provided as tertiles, therefore not able to use per-protocol data in meta-analysis)

SES: Ethnicity data provided

Overall: Black $n=146$; white $n=280$; Hispanic $n=166$; other $n=88$

Gp A: Black $n=76$; white $n=140$; Hispanic $n=81$; other $n=41$

Gp B: Black $n=70$; white $n=140$; Hispanic $n=85$; other $n=47$

Adverse events: Quote: "No study-related serious adverse events occurred" 
Diabetes rescue therapy required by $1.7 \%$ in $\mathrm{Gp} \mathrm{A}(4 / 241)$, and $2.1 \%$ in $\mathrm{Gp} \mathrm{B}(5 / 236)$ during the trial

Change in medication from baseline required by $45.0 \%$ in Gp A (105/233), and $40.2 \%$ in Gp B (92/229)

HbA1c assessment method: Whole-blood samples iced and analysed within 4 days by high-performance liquid chromatography (Tosoh HPLC G7 Glycohemoglobin Analyzer, Tosoh Medics Inc)

Conflict of interests: No conflict declaration from lead author (Dr Engebretson), but available for others:

Quote: "Dr Gelato reported receiving travel/meeting expenses from the Endocrine Society. Dr Seaquist reported serving as a board member and President Elect of Science and Medicine for the American Diabetes Association; serving as a consultant for AMG Medical, sanofi-aventis, SkyePharma, and Merck; receiving grants or grants pending from the American Diabetes Association, Eli Lilly, and the National Institutes of Health; and receiving payment for lectures from the Japan Diabetes Society, the American Diabetes Association, Intellyst Medical Education, Pediatric Academic Societies, the Association of Specialty Professors, and the International Society for Neurochemistry. Dr Lewis reported receiving a grant or grant pending from Novo Nordisk. Dr Katancik reported serving as a consultant for the Texas Healthy Baby Initiative 2011 and receiving a grant or grant pending, and travel/meeting expenses, from Zimmer Dental. Dr Paquette reported serving as a board member for Colgate-Palmolive; receiving a speakers honorarium from Colgate-Palmolive; and serving as a consultant for MIS Implant Technologies"

Trial ID: NCT00997178 (trial referred to as Diabetes and Periodontal Therapy Trial (DPTT))

\section{Risk of bias}

\begin{tabular}{lll}
\hline Bias & Authors' judgement & Support for judgement \\
\hline $\begin{array}{l}\text { Random sequence genera- } \\
\text { tion (selection bias) }\end{array}$ & Low risk & $\begin{array}{l}\text { Quote: "Randomization was conducted centrally by the CC using a site-specific } \\
\text { randomization assignment sequence generated prior to the start of the study. } \\
\text { Assignments to the Treatment and Control Groups were created through a } \\
\text { custom computer program using a permuted block randomization scheme } \\
\text { stratified by Clinical Site using block sizes of 2, } 4 \text { or } 6 "\end{array}$ \\
\hline
\end{tabular}

\section{Allocation concealment Low risk} (selection bias)
Quote: "..randomization assignments by individual participant were accessible in Velos eResearch only to the necessary CC personnel and the Clinical Site Coordinators. Participant IDs did not contain treatment assignment codes"

"Once eligibility for an individual was confirmed, the CC Study Coordinator generated the randomization assignment electronically and notified the Clinic Coordinator by email or fax. The Clinic Coordinator then contacted the participant with the treatment group assignment. No other Clinical Site personnel other than the Study Therapist were informed of the assignments"

\begin{abstract}
Quote: "Double masking would have required us to provide some type of "sham" periodontal therapy to control participants, which, to the best of our knowledge, had not been done in any previous trial in periodontology"

"Periodontal therapy also frequently results in gingival (gum) recession and tooth sensitivity, especially to hot and cold temperatures. Treatment also removes the discolored calcified deposits that form at and just beneath the gum line. These signs and symptoms, which can be readily noticed by patients, would not be expected following some type of "sham" treatment. Thus, it is unlikely that the provision of a sham treatment would adequately mask control participants either"
\end{abstract}


Engebretson 2013 (Continued)

Blinding of clinical opera- High risk Quote: "An endpoint of treatment is the complete removal of hard and soft detor posits from the tooth and root surfaces. Thus it is not possible to mask therapists"

\begin{tabular}{|c|c|c|}
\hline $\begin{array}{l}\text { Incomplete outcome data } \\
\text { (attrition bias) }\end{array}$ & Low risk & $\begin{array}{l}93 \% \text { completed the study (476/514), similar retention across both arms Gp A: } \\
240 / 257(93.4 \%) ; \text { Gp B: } 236 / 257(91.8 \%)\end{array}$ \\
\hline All outcomes & & ITT analysis of HbA1c data. Periodontal data provided per-protocol analysis \\
\hline
\end{tabular}

\begin{tabular}{ll}
\hline $\begin{array}{l}\text { Selective reporting (re- } \\
\text { porting bias) }\end{array}$ & Low risk
\end{tabular}

Other bias Unclear risk Conflict of interests declaration reported for all authors except lead author

Gay 2014

Methods

Trial design: 2 -arm, single-centre, parallel-design RCT

Location: USA

Setting: Hospital

Number of centres: 1, University of Texas Health Science Center, Houston, Texas

Recruitment period: Not reported.

Funding source: Quote: "This study is funded by National Institutes of Health Clinical and Translational Award ULI RR024148 and KL2 RR024149 from the National Center For Research Resources"

Participants

Inclusion criteria: >18 yrs old; diagnosed T2 DM; possessing $\mathrm{HbA} 1 \mathrm{c}$ value $>6.5 \%$ at screening (although initial values of $5.7-6.5 \%$ were included if taking hypoglycaemic medication: $n=16$ (note: unsure of allocation between groups)); Hispanic; presence of local or general severe chronic periodontitis (AAP criteria)

Exclusion criteria: Smokers; dental treatment within prior 12 months; systemic antibiotics within 6 months of recruitment (not specified if a pre- or post-recruitment requirement)

Age at baseline: Overall: mean 52.8 yrs (SD 9.7); Gp A: mean 51.5 yrs (SD 9.0); Gp B: 54.0 yrs (SD 10.2). No $P$ value reported

Sex (M:F): Overall M55:F71; Gp A: M30:F36; Gp B: M25:F35. No P value reported

Tobacco use: Smokers were excluded from participation in the trial

Weight: Not reported

BMI: Not reported

Alcohol consumption: Not reported

Diabetes type: All T2 DM

Duration since diabetes diagnosis: Not reported

Metabolic control: Poor mean HbA1c at baseline Mean HbA1c at baseline: Gp A: 9.00\% (SD 2.30); Gp B: 8.40\% (SD 2.00)

Antidiabetic therapy: All except 26 patients ( $21 \%$ of 126 participants) were in receipt of "diabetic treatment" without further description: Gp A 78.8\% ( $n=52)$; Gp B $80.0 \%(n=48)$. Of diabetic treatment recipients, 21 patients were on insulin therapy: Gp A: $21 \%(n=14) ; G p$ B: $12 \%(n=7)$

Other investigations: Distance from free gingival margin to cementoenamel junction (FGM-CEJ) 
Other medical conditions: Not reported

Number randomised: 154 (Gp A n $=77 ;$ Gp B n $=77$ )

Number evaluated: $126(\mathrm{Gp} \mathrm{A} \mathrm{n}=66 ; \mathrm{Gp} \mathrm{B} n=60)$

Note: All data (including baseline) only presented for evaluated patients, rather than those randomised

Attrition: Gp A: dropped out $n=2$; lost to follow-up $n=8$ (1 patient not accounted for); Gp B: dropped out $n=12$; lost to follow-up $n=2$; excluded for unreliable data $n=2$ ( 1 patient not accounted for)

Interventions

Comparison: SRP + OHI (x 2) versus OHI

Gp A ( $n=77)$ : OHI at baseline (including modified Bass technique, interdental brush/floss use), + SRP 4-6 weeks later (ultrasonic scaler, Gracey curettes, on 2 quadrants, local anaesthetic, by 2 calibrated periodontists) when $\mathrm{OHI}$ repeated

Gp B ( $n=77)$ : OHI at baseline (including modified Bass technique, interdental brush/floss use), + repeat OHI 4-6 weeks later

Duration of follow-up: 4 months

\section{Outcomes}

Primary: $\mathrm{HbA1c}$ (at baseline and 4 months)

Secondary: BOP, PD and CAL (at baseline and 1 month)

Notes Sample size calculation: 123 participants required ( $90 \%$ power: 2 -sided t-test, .05 type I error). Accounting for attrition rate of $20 \%$, planned sample size was 154 (77 in each arm)

Data analysis: Per-protocol

SES: Not reported specifically except that all participants were of Hispanic origin

Adverse events: Not reported

Change in medication from baseline required by Gp A: $27.3 \%(n=18) ; \mathrm{Gp} \mathrm{B}: 21.7 \%(n=13)$

HbA1c assessment method: Afinion AS100 Analyzer. High value samples run in duplicate, and several other samples run in duplicate for compliance

Conflict of interests: Authors declare no conflict of interests

Trial ID: NCT01128374

\section{Risk of bias}

\begin{tabular}{lll}
\hline Bias & Authors' judgement & Support for judgement \\
\hline $\begin{array}{l}\text { Random sequence genera- } \\
\text { tion (selection bias) }\end{array}$ & Low risk & $\begin{array}{l}\text { Computer-randomised sequence generation } \\
\text { Quote: "Permuted blocks randomization with varying block sizes using Stata } \\
11 \text { was performed by a statistician (DT) to generate allocation sequences" }\end{array}$ \\
\hline $\begin{array}{l}\text { Allocation concealment } \\
\text { (selection bias) }\end{array}$ & Unclear risk & $\begin{array}{l}\text { Quote: "These sequences were used by the research coordinator (AC) to recruit } \\
\text { and blindly randomize } 154 \text { participants either to a control ( } n=77) \text { or experi- } \\
\text { mental group ( } n=77) \text { with a } 1: 1 \text { allocation ratio" }\end{array}$ \\
& $\begin{array}{l}\text { Comment: "Blindly randomized" does not infer adequate allocation conceal- } \\
\text { ment }\end{array}$ \\
\hline Blinding of participants & Unclear risk & Not reported \\
\hline
\end{tabular}


Gay 2014 (Continued)
Blinding of clinical opera-
Unclear risk
Not reported
tor

Incomplete outcome data High risk (attrition bias)

All outcomes
All data (including baseline) only presented for evaluated $(n=126)$ patients, rather than those randomised $(n=154)$

1 patient from each group not accounted for Attrition: Gp A: dropped out $n=2$; lost to follow-up $n=8$ (1 patient not accounted for); Gp B: dropped out $n=12$; lost to follow-up $n=2$; excluded for unreliable data $n=2$ (1 patient not accounted for)

Per-protocol analysis: not all participants analysed in groups randomised to, regardless of intervention actually received

\begin{tabular}{|c|c|c|}
\hline $\begin{array}{l}\text { Selective reporting (re- } \\
\text { porting bias) }\end{array}$ & Unclear risk & $\begin{array}{l}\text { All initially stated outcomes reported on in results/tables, albeit only including } \\
\text { those evaluated. No adverse events reported }\end{array}$ \\
\hline
\end{tabular}

Other bias Low risk No other apparent biases

Location: Poland

Setting: Not reported

Number of centres: Not reported, but assumed to be single centre due to single examiner and small sample size

Recruitment period: Not reported

Funding source: Medical University of Silesia, Katowice, Poland moderate, localized or generalized CP. Each patient had at least 4 non-adjacent sites with PD $>=4 \mathrm{~mm}$

Exclusion criteria: Previous dental prophylaxis and periodontal treatment within 6 months of the initial visit; known hypersensitivity to tetracyclines; smoking; antibiotic therapy within the 3 months of the initial visit; chronic therapy with non-steroidal anti-inflammatory drugs or immunosuppressive medications and administration of medications that can interfere with doxycycline (anticoagulants and contraceptive drugs); systemic diseases, which can have an influence on periodontal status or immunologic profile (osteoporosis, autoimmune diseases, primary-,or secondary immunodeficiency); pregnancy; or lactation. No clinical signs of aggressive periodontal disease

Age at baseline: Overall mean 56.8 yrs (SD 8.4); Gp A mean 57.6 yrs (SD 8.0); Gp B mean 56.0 yrs (SD 9.0) $(P=0.59)$

Sex (M:F): Overall M16:F18; Gp A M7:F10; Gp B M9:F8 ( $P=0.73)$

Tobacco use: Only non-smokers enrolled

Alcohol consumption: Not reported

Diabetes type: $T 2 \mathrm{DM}$

Duration since diabetes diagnosis: Gp A: mean 6.7 yrs (SD 6.5); Gp B: mean 9.4 yrs (SD 8.1) $(P=0.24)$

Metabolic control: Good-fair mean $\mathrm{HbA1c}$ at baseline

Mean HbA1c at baseline: Gp A 6.9\% (SD 1.2); Gp B 7.3\% (SD 2.0) $(P=0.36)$ 
Antidiabetic therapy: Quote: "All patients received optimal diabetic treatment including diet regimen, insulin supplementation, and/or oral hypoglycemic drugs"

Other clinical investigations: GCF, MMP-8

Other medical conditions: As stated in exclusion criteria

BMI: Gp A: 31.0 (SD 4.9); Gp B: 29.8 (SD 5.0) (P = 0.93)

Number randomised: 34

Number evaluated: Assumed 34

Comparison: SRP + OHI + doxycycline tablet (20 mg bid) versus SRP + OHI + placebo tablet
Gp A $(n=17): S R P+O H I+S D D:$ subantimicrobial dose doxycycline 20 mg bid 3 months
Gp B $(n=17): S R P+O H I+$ placebo bid 3 months

Duration of follow-up: 3 months

\begin{tabular}{ll}
\hline Outcomes & Primary: $\mathrm{HbAlc}$ (at baseline and 3 months) \\
& Secondary: CAL, BOP, PPD (at baseline and 3 months)
\end{tabular}

Notes Sample size calculation: "Based on previous information from a pilot study recently conducted by our research group, using data relative to the mean difference between groups and standard deviation 3 months after periodontal treatment (unpublished data). Using difference in clinical parameter ( $P D>=$ $4 \mathrm{~mm}$ ) as primary outcome, with an expected mean difference between groups after the therapy of 0.5 $\mathrm{mm}$ and an expected standard deviation of $0.5 \mathrm{~mm}$, it was estimated that at $80 \%$ power and a level of significance of 0.05 , the sample size should be 16 subjects per group. To allow the possible drop outs, 34 patients (17 per group) were finally recruited"

(Note: Principal author supplied further unpublished data, including mean (SD) data)

Data analysis: Assumed ITT

HbA1c assessment method: Turbidimetric inhibition immunoassay (Cobas Integra 400 plus; Roche Diagnostics Polska)

Adverse events: No adverse effects reported by participants

SES: Not reported

Conflict of interests: Not reported

\section{Risk of bias}

\begin{tabular}{lll}
\hline Bias & Authors' judgement & Support for judgement \\
\hline $\begin{array}{l}\text { Random sequence genera- } \\
\text { tion (selection bias) }\end{array}$ & Low risk & Did not specify computerised per se, but did mention randomised blocks \\
\hline $\begin{array}{l}\text { Allocation concealment } \\
\text { (selection bias) }\end{array}$ & Low risk & Investigators not involved in assignment of drug containers \\
\hline Blinding of participants & Low risk & Quote: "indistinguishable placebo" \\
\hline $\begin{array}{l}\text { Blinding of clinical opera- } \\
\text { tor }\end{array}$ & Low risk & All personnel blinded "indistinguishable placebo" \\
\hline $\begin{array}{l}\text { Incomplete outcome data } \\
\text { (attrition bias) }\end{array}$ & Unclear risk & $\begin{array}{l}\text { Comment: Insufficient information to judge. No indication of the numbers } \\
\text { used in each analysis }\end{array}$ \\
\hline
\end{tabular}

Treatment of periodontal disease for glycaemic control in people with diabetes mellitus (Review) 
Gilowski 2012 (Continued)

All outcomes

\section{Analysis assumed to have been ITT, but not specifically reported}

\begin{tabular}{lll}
\hline $\begin{array}{l}\text { Selective reporting (re- } \\
\text { porting bias) }\end{array}$ & High risk & CAL data not reported although measured \\
\hline Other bias & Low risk & No other apparent biases \\
\hline
\end{tabular}

Grossi 1997

Methods Trial design: 5-arm, single-centre, parallel-design RCT

Location: USA

Setting: Not reported

Number of centres: 1

Recruitment period: Not stated

Funding source: Grants from 'USPHS' and National Institute of Dental Research and equipment donated by Eastman Kodak

Inclusion criteria: Non-insulin dependent diabetics from the Gila River Indian Community, aged 25 to 65 years, with moderate to severe periodontitis

Exclusion criteria: Patients on renal dialysis or presenting with diabetic complications requiring hospitalisation

Age at baseline: Not reported

Sex (M:F): Overall M32:F81. "..patients [...] stratified by duration of diabetes (below and above 10 years duration), insulin use, and sex and randomly assigned to one of the 5 treatment groups. Each of the groups had at least one third males"

Tobacco use: Not reported

Alcohol consumption: Not reported

Diabetes type: T2 DM

Duration since diabetes diagnosis: Not reported

Metabolic control: Poor mean HbA1c at baseline

Mean HbA1c at baseline: Gp A: 10.4\% (SD 2.6); Gp B: 10.4\% (SD 1.9); Gp C: 10.3\% (SD 2.6); Gp D: 10.5\% (SD 2.0); Gp E: 9.3\% (SD 2.7)

(Note: Means provided by study author (we requested SDs) differ from published figures (means and SEs). Content provided by author used where available)

Antidiabetic therapy: All in receipt of oral hypoglycaemic medication. Also states groups were stratified by insulin use, but no further detail provided

Other clinical investigations: Detection of Porphyromonas gingivalis

Other medical conditions: Not reported

Number randomised: 113

Number evaluated: $106 / 7$

(Depends on outcome: HbA1c: 106; CAL/GI/PI/PPD: 107) 
Grossi 1997 (Continued)

Interventions

Comparison: SRP + water rinse + doxycycline versus SRP + chlorhexidine + doxycycline versus SRP + iodine + doxycycline versus SRP + chlorhexidine + placebo versus SRP + water rinse + placebo

Gp A ( $n=18): S R P+$ water rinse + doxycycline (100 mg daily for 14 days)

Gp B ( $n=22):$ SRP + chlorhexidine $(0.12 \%)+$ doxycycline (100 mg daily for 14 days)

Gp C ( $n=21):$ SRP + iodine ( $0.05 \%$ povidone iodine $)+$ doxycycline (100 mg daily for 14 days $)$

Gp D ( $n=27):$ SRP + chlorhexidine $(0.12 \%)+$ placebo (daily for 14 days)

Gp E ( $n=24): S R P+$ water rinse + placebo (daily for 14 days)

All participants received ultrasonic bactericidal curettage (UBC) performed with an ultrasonic device, with continuous irrigation with an antimicrobial solution, in 2 sessions, 1 week apart at baseline (half of mouth was treated at each session)

Duration of follow-up: 6 months

$\begin{array}{ll}\text { Outcomes } & \text { Primary: } \mathrm{HbAlc} \text { (at baseline, } 3 \text { and } 6 \text { months) } \\ & \text { Secondary: } \mathrm{CAL}, \mathrm{PD}, \mathrm{PI} \text { and } \mathrm{GI} \text { (at baseline, } 3 \text { and } 6 \text { months) }\end{array}$

Notes

Sample size calculation: Not reported

Data analysis: Per-protocol

SES: Not specifically detailed, but all participants were Native Indian residents of the Gila River Indian Community (of Pima or Pima/Papago heritage), in Arizona, USA

Adverse events: Not reported

HbA1c assessment method: Biorad high performance liquid chromatography (HPLC)

Conflict of interests: Not reported

Data provided by email from Bob Genco. Group ns not clearly detailed in study paper, instead derived from provided data

\section{Risk of bias}

\begin{tabular}{lll}
\hline Bias & Authors' judgement & Support for judgement \\
\hline $\begin{array}{l}\text { Random sequence genera- } \\
\text { tion (selection bias) }\end{array}$ & Unclear risk & $\begin{array}{l}\text { Quote: "randomisation was stratified by duration of diabetes }(>\text { or }<10 \text { years), } \\
\text { insulin use, and sex." Method of sequence generation not described }\end{array}$ \\
\hline $\begin{array}{l}\text { Allocation concealment } \\
\text { (selection bias) }\end{array}$ & Unclear risk & Not reported \\
\hline $\begin{array}{l}\text { Blinding of participants } \\
\text { Blinding of clinical opera- } \\
\text { tor }\end{array}$ & Hnclear risk risk & Not reported, perhaps not possible \\
\hline $\begin{array}{l}\text { Incomplete outcome data } \\
\text { (attrition bias) } \\
\text { All outcomes }\end{array}$ & High risk & Not possible \\
\hline
\end{tabular}


Grossi 1997 (Continued)

Selective reporting (re- Unclear risk HbAlc not reported in publication, although obtained directly from authors porting bias) and no estimates of variance reported for PD or plaque, numbers in each group not reported. Adverse events not reported

Other bias High risk Imbalance of HbA1c between groups at baseline. Antidiabetic therapy use not reported

Location: Iran

Setting: Hospital

Number of centres: 1, Diabetes Research Center, Yazd City

Recruitment period: Not reported

Funding source: Not reported

\section{Participants}

Inclusion criteria: Patients with type 2 diabetes and proper blood glucose control $(\mathrm{HbA} 1 \mathrm{c} \leq 7 \%)$ and chronic periodontitis with 3 regions probe depth more than $4 \mathrm{~mm}$ and less than $7 \mathrm{~mm}$

Exclusion criteria: Patients treated with anti-inflammatory agents; systemic antibiotics within 3 months before the start of study; periodontal treatment in past 6 months; smokers; acute medical conditions; less than 8 teeth in month; and pregnant or breast-feeding women

Age at baseline: Not reported

Sex (M:F): Not reported

Tobacco use: Not reported but smoking reported as an exclusion criteria

Alcohol consumption: Not reported

Diabetes type: All participants had diabetes type 2

Duration since diabetes diagnosis: Not reported

Metabolic control: Good mean $\mathrm{HbAlc}$ at baseline Mean HbA1c at baseline: Gp A: 6.48\% (SD 0.51) Gp B: 6.44\% (SD 0.35)

Antidiabetic therapy: Not reported.

Other investigations: Cholesterol, LDL, HDL, triglyceride

Other medical conditions: N/A

Number randomised: 30

Number evaluated: 30

Gp A ( $n=15)$ : SRP with topically applied tetracycline gel (5\%)

Gp B ( $n=15):$ SRP (standard periodontal care)

Duration of follow-up: 3 months

Outcomes Primary: HbA1c (at baseline and 3 months)

Treatment of periodontal disease for glycaemic control in people with diabetes mellitus (Review) 
Secondary: GI, PI, and PPD (at baseline and 3 months)

Notes

Sample size calculation: Not reported

Data analysis: ITT

HbA1c assessment method: Not reported

Trial ID: IRCT2013092614774N1 (www.irct.ir/searchresult.php?id=14774\&number=1)

Conflict of interests: Not reported

Adverse events: Not reported

SES: Not reported

Study data translated by Farhad Shokraneh - January 2015

\section{Risk of bias}

\begin{tabular}{lll}
\hline Bias & Authors' judgement & Support for judgement \\
\hline $\begin{array}{l}\text { Random sequence genera- } \\
\text { tion (selection bias) }\end{array}$ & Unclear risk & $\begin{array}{l}\text { Not reported. (Protocol (see URL above) simply refers to an "interventional } \\
\text { randomized clinical trial" "randomly divided 15 patients") }\end{array}$ \\
\hline $\begin{array}{l}\text { Allocation concealment } \\
\text { (selection bias) }\end{array}$ & Unclear risk & Not reported. \\
\hline $\begin{array}{l}\text { Blinding of participants } \\
\text { Blinding of clinical opera- } \\
\text { tor }\end{array}$ & Unclear risk & Not reported \\
\hline $\begin{array}{l}\text { Incomplete outcome data } \\
\text { (attrition bias) }\end{array}$ & Low risk & Not reported \\
All outcomes & No losses to follow-up. ITT analysis \\
\hline $\begin{array}{l}\text { Selective reporting (re- } \\
\text { porting bias) }\end{array}$ & Unclear risk & $\begin{array}{l}\text { All recorded outcomes were reported on within the results section, however, } \\
\text { no adverse events reported }\end{array}$ \\
\hline $\begin{array}{l}\text { Other bias } \\
\text { Und }\end{array}$ & Unclear risk & $\begin{array}{l}\text { No way to verify if other biases exist due to translation of data extraction com- } \\
\text { ponents }\end{array}$ \\
\hline
\end{tabular}

Jones 2007

Methods Trial design: 2-arm, multicentre, parallel-design RCT (at 4 months)

Location: USA

Setting: Primary care

Number of centres: 4, New England

Recruitment period: Not stated

Funding source: Grants from Veterans Affairs Health Services Research and Development Service and Boston University (VA HSR\&D QUERI DII-99.206 and NIH K24 DE00419). Dentsply International provided ultrasonic scalers, and Colgate Oral Pharmaceuticals provided the Gluconate rinse (PerioGards) 
Jones 2007 (Continued)

Participants
Inclusion criteria: A repeat $\mathrm{HbA1c}$ of $8.5 \%$ or above; a minimum of 8 natural teeth; periodontal treatment need as evidenced by the Community Periodontal Index of Treatment Need CPITN scores of 3 or 4 in at least 2 sextants on examination; and sufficient health and willingness to complete the 12-16month study

Exclusion criteria: Grave medical or psychiatric illness or severe immune compromise (eg HIV or cancer)

Age at baseline: Mean 58.36 yrs. Gp A: 57.79 yrs; Gp B: 58.96 yrs. 4-month group 58.08, 12-month group 58.39

Sex (M:F): Overall: M97\%:F3\%; Gp A: M100\%:F0\%; Gp B: M94\%:F6\%

Tobacco use: Overall: 24\%; Gp A: 29.5\%; Gp B: $18.8 \%$

Alcohol consumption: Overall: 1.8 drinks p/wk (SD 5); Gp A: 2.2 drinks p/wk (no SD); Gp B: 1.43 drinks p/wk (no SD)

Diabetes type: Assumed majority T2 DM

Quote: "Because all participants were veterans whose admission to military service was on the basis of their health, and thus developed diabetes after the beginning of military service, we reasoned that the vast majority of them had Type 2 diabetes"

Duration since diabetes diagnosis: Gp A 11.4 yrs; Gp B 14.1 yrs (no SDs provided by group)

Metabolic control: Poor mean $\mathrm{HbA1c}$ at baseline

Mean HbA1c pre-baseline: Gp A: 10.07\%; Gp B: 10.29\%

Antidiabetic therapy: All in receipt of oral hypoglycaemic medications, insulin, or combination

Other medical conditions: Many co-morbidities (co-morbidity index: Gp A: 5.95; Gp B: 6.11), high levels of hypertension, hypercholesterolaemia, obesity, atherosclerosis

Number randomised: 193

Number evaluated: 165 (Gp A: 82; Gp B: 83)/132 depending on outcome

Interventions

\section{Comparison: SRP + doxycycline + chlorhexidine rinse versus usual treatment}

Gp A ( $n=98)$ : SRP + doxycycline (100 mg qid for 14 days) + chlorhexidine rinse $(0.12 \%$ twice daily for 4 months)

Gp B ( $\mathrm{n}=95)$ : Usual treatment (described only as "usual medical and dental care")

Duration of follow-up: 4 months

Outcomes

Primary: Change in HbAlc (not fully reported)

Secondary: $\mathrm{Gl}$, gingival recession

Notes

Sample size calculation: Quote: "The study was designed to have 300 participants. Allowing for $33 \%$ attrition, we expected 200 patients studied, 100/group. We anticipated $80 \%$ power to detect a moderate-sized effect ( $E S \delta=0.40$ ) of the intervention in 2 -sided tests at the $5 \%$ level. For the analysis at 4 months comparing the proportion of patients in Early Treatment and Usual Care groups who experienced a greater than $1 \%$ drop in their HbA1c levels, we expected similar power"

Data analysis: Per-protocol

\section{Adverse events:}

Chlorhexidine: Disturbance in taste (15\%); tooth staining (13.6\%); sore mouth/tongue irritation (5\%); swelling of lips, face, tongue and throat also reported in a small number of participants. Also shortness of breath

Doxycyline: Diarrhoea (7.1\%); abdominal pain (3.6\%); nausea (2.9\%)

Treatment of periodontal disease for glycaemic control in people with diabetes mellitus (Review) 
Jones 2007 (Continued)

"Compliance with the study drug regimen was not universal. Eighty-three percent used both chlorhexidine and doxycycline, another $8 \%$ used chlorhexidine only, and $7 \%$ used doxycycline only. Thus, over $90 \%$ in the treatment group used each study drug. Among users of chlorhexidine, 17 participants reported less than daily use, 19 reported daily use, and 29 reported twice daily use. One chlorhexidine user had four bottles left, nine had two to three bottles left, 16 had one left, and 41 used all the chlorhexidine. Among doxycycline users 50 reported using all the pills, two had 10 pills left (of 14), and five had more than 10 pills left"

SES: Race is reported, although only as \% of white participants: Overall: $97 \%$; Gp A: $84 \%$; Gp B: 79\%

HbA1c assessment method: Not reported

Conflict of interests: Not reported

Means data for analysis provided by lead author in 2007

\section{Risk of bias}

Bias Authors' judgement Support for judgement

Random sequence genera- Low risk tion (selection bias)
Quote: "We used PROC PLAN in Statistical Analysis Systems (SAS) Version 8.1, Cary, NC, USA) to obtain 12 blocks of eight, using a seed of 020348. Group assignments were put on white cards and sealed in white envelopes and numbered consecutively. Study staff took the top envelope to assign study group"

\begin{tabular}{lll}
\hline $\begin{array}{l}\text { Allocation concealment } \\
\text { (selection bias) }\end{array}$ & Low risk & See above \\
\hline Blinding of participants & High risk & Participants knew which group they are allocated to \\
\hline $\begin{array}{l}\text { Blinding of clinical opera- } \\
\text { tor }\end{array}$ & High risk & $\begin{array}{l}\text { Quote: "...by seeking physicians' concurrence, in essence we notified each par- } \\
\text { ticipant's primary care provider that his or her patient's diabetes was under } \\
\text { poor control. Because of this notification,some providers likely became more } \\
\text { aggressive in treating these patients" }\end{array}$ \\
\hline
\end{tabular}

Incomplete outcome data High risk
(attrition bias) All outcomes

193 participants recruited, 28 excluded after randomisation for reasons not related to interventions. Numbers from each group not reported. 165 in study providing baseline data then 33 withdrawals, reasons given but not by group

Potentially, such high drop-out rates within the short study duration may reflect the reported adverse events experienced by Gp A (relating to doxycycline and chlorhexidine)

Per-protocol analysis: not all participants analysed in groups randomised to, regardless of intervention actually received

Selective reporting (re- High risk
porting bias)
No mean HbA1c values at 4 months reported, only 2 dichotomous outcomes. No reporting of SD for each group, only overall reported Author supplied means and SDs in correspondence

Adverse events only reported for Gp A

All characteristics data (including baseline) only presented for evaluated patients (varies for each characteristic) $(n=154-165)$, rather than those randomised $(n=193)$

1 patient from each group not accounted for
High risk
Baseline differences with respect to smoking, history of stroke, TIAs, diabetes with nephropathy 
Location: Japan

Setting: Hospital

Number of centres: 5 diabetic clinics: Tokyo Medical and Dental University Hospital, Kagoshima University Medical and Dental Hospital, Aichi Gakuin University Dental Hospital, Tokyo Medical University Hospital and Kyoto Prefecture Medical University Hospital.

\section{Recruitment period: Not reported}

Funding source: Supported by Grants-in Aid from the Ministry of Health and Welfare of Japan (H16Iryo-020) and the Mitsui Sumitomo insurance foundation

Participants

Inclusion criteria: Aged 39-75 years, $\mathrm{HbA1c} 6.5-10.0 \%$; at least 11 remaining teeth, at least 2 pocket sites with probing depth $4 \mathrm{~mm}$ or more (indicated as mild to severe periodontitis), no periodontal treatment during the preceding 6 months

Exclusion criteria: Severe diabetic complications; evidence of systemic diseases other than diabetes as a risk factor for periodontitis; systemic antibiotics during the preceding 3 months; pregnancy or lactation; allergy to tetracycline; smoking; modifications in the treatment of diabetes during the preceding 2 months

Age at baseline: Overall: 59.7 yrs (SD 7.4); Gp A: mean 60.3 yrs (SD 9.9); Gp B: mean 59.0 yrs (SD 4.8)

Sex (M:F): Overall: M27:F22; Gp A: M21:F11; Gp B: M6:F11

Tobacco use: Non-smokers

Alcohol consumption: Not stated

Diabetes type: T2 DM

Duration since diabetes diagnosis: Gp A: 11.3 yrs (SD 6.4); Gp B: 8.8 yrs (SD 7.5)

Metabolic control: Good mean $\mathrm{HbA1c}$ at baseline Mean HbA1c at baseline: Gp A: 7.2 (SD 0.9); Gp B: 6.9 (SD 0.9)

Antidiabetic therapy: All in receipt of oral hypoglycaemic medication, insulin, or diet

Diet: Overall: $n=3 ;$ Gp A: $n=1 ; G p$ B: $n=2$

Oral hypoglycaemic medication: Overall: $\mathrm{n}=27$; Gp A: $\mathrm{n}=15 ; \mathrm{Gp} \mathrm{B}: \mathrm{n}=12$

Insulin: Overall: $\mathrm{n}=19 ; \mathrm{Gp}$ A: $\mathrm{n}=16 ; \mathrm{Gp} \mathrm{B}: \mathrm{n}=3$

Other medical conditions: None reported

Number randomised: 49 (Gp A 32; Gp B 17)

Number evaluated: 49

\section{Comparison: SRP + minocycline + $\mathrm{OHI}$ versus $\mathrm{OHI}$}

Gp A ( $n=32)$ : Mechanical debridement of the subgingival plaque and calculus was performed using piezoelectric ultrasonic scalers, and $10 \mathrm{mg}$ of minocycline ointment (Periofil1, Showa Yakuhin Co., Tokyo, Japan) was administered topically in every periodontal pocket at the end of each visit. The in- 
tensive periodontal treatment was completed over the course of 4 visits within 2 months. Additional periodontal treatment including instructions for brushing, supra- and sub-gingival debridement without topical administration of antibiotics were performed, if necessary

Gp B ( $=17)$ : Instructions for brushing their teeth, including the use of interproximal cleaning aids, such as floss and interdental brushes, depending on their individual needs

After the completion of 2 months of intensive period periodontal treatment, all participants visited the respective medical and dental clinics at 1, 3 and 6 months

Duration of follow-up: 6 months

$\begin{array}{ll}\text { Outcomes } & \text { Primary: HbAlc at } 1 \text { month, } 3 \text { months and } 6 \text { months } \\ & \text { Secondary: Change in PPD at } 1 \text { month (Delta PPD), change in BOP at } 1 \text { month (Delta BOP) and inter- } \\ & \text { vention of periodontal treatment on the change in HbAlc at } 6 \text { months }\end{array}$

Notes

Sample size calculation: Not reported

Data analysis: ITT

HbA1c assessment method: High-performance liquid chromatography (Kyotokagaku Co, Japan)

Adverse events: Not reported

SES: Not reported

Conflict of interests: Authors declare no conflict of interests

\begin{tabular}{lll}
\hline Risk of bias & & \\
\hline Bias & Authors' judgement & Support for judgement \\
\hline $\begin{array}{l}\text { Random sequence genera- } \\
\text { tion (selection bias) }\end{array}$ & Unclear risk & $\begin{array}{l}\text { Quote: "randomly allocated by envelope method" - method of sequence gen- } \\
\text { eration not described }\end{array}$ \\
\hline $\begin{array}{l}\text { Allocation concealment } \\
\text { (selection bias) }\end{array}$ & High risk & $\begin{array}{l}\text { Envelope method. Dentists knew the allocations to each group (from corre- } \\
\text { spondence with the author) }\end{array}$ \\
\hline $\begin{array}{l}\text { Blinding of participants } \\
\text { Blinding of clinical opera- } \\
\text { tor }\end{array}$ & High risk risk & Not possible \\
\hline $\begin{array}{l}\text { Incomplete outcome data } \\
\text { (attrition bias) } \\
\text { All outcomes }\end{array}$ & Low risk & Not possible \\
\hline $\begin{array}{l}\text { Selective reporting (re- } \\
\text { porting bias) }\end{array}$ & Unclear risk & All randomised participants included in outcome evaluation. ITT analysis \\
\hline \begin{tabular}{l} 
Other bias \\
\hline
\end{tabular} & Low risk & $\begin{array}{l}\text { HbAlc not reported by group but details later supplied by the lead author. Ad- } \\
\text { verse events not reported }\end{array}$ \\
\hline
\end{tabular}

Kiran 2005

Methods Trial design: 2-arm, single-centre, parallel-design RCT

Location: Turkey 
Kiran 2005 (Continued)
Setting: Hospital Endocrinology
Recruitment period: Not reported
Funding source: Not reported

Number of centres: 1, Ankara University Faculty of Medicine, Department of Metabolic Diseases and

Participants

Inclusion criteria: Patients with type 2 DM with glycated haemoglobin (HbA1c) values: $6 \%-8 \%$; creatinine values $01.4 \mathrm{mg} / \mathrm{dl}$; liver function tests not $>3 \times$ the normal range

Exclusion criteria: Major diabetic complications; systemic antibiotics administered within prior 3 months; periodontal treatment within prior 6 months

Sex (M:F): Overall: M18:F26; Gp A: M10:F12; Gp B: M8:F14

Age at baseline: Overall 54.39 yrs (SD 11.27); Gp A: mean 55.95 yrs (SD 11.21); Gp B: mean 52.82 yrs (SD 12.27)

Tobacco use (daily): Overall: $\mathrm{n}=7$ (15.9\%); Gp A $n=5$ (22.7\%); Gp B n = 2 (9.1\%)

Alcohol consumption: Not reported

Diabetes type: $\mathrm{T} 2 \mathrm{DM}$

Duration since diabetes diagnosis: Overall mean 8.68 yrs (SD 7.18). Gp A: 9.32 yrs (SD 11.21); Gp B: 8.05 yrs (SD 5.90)

Metabolic control: Good-fair mean HbA1c at baseline Mean HbA1c at baseline: Gp A: 7.31\% (SD 0.74); Gp B: 7.00\% (SD 0.72)

Antidiabetic therapy: All in receipt of oral hypoglycaemic medication (Gp A: 64\%; Gp B: 72\%), insulin (Gp A: 9\%; Gp B: 9\%), diet (Gp A: 9\%; Gp B: 5\%) or combination (Gp A: 18\%; Gp B:14\%). No P values presented

Other clinical investigations: Gingival recession; fasting plasma glucose; 2-hour post-prandial glucose; total cholesterol; triglyceride; HDL-cholesterol (HDL); LDL-cholesterol (LDL); microalbuminurea

Other medical conditions: None reported

Number randomised: 44

Number evaluated: 44

Interventions

\section{Comparison: SRP + OHI versus no intervention}

$\mathrm{Gp} \mathrm{A}(\mathrm{n}=22): \mathrm{OHI}$ and full mouth SRP performed under local anaesthesia

Gp B ( $n=22)$ : No periodontal treatment during study period (delayed treatment offered, if required, after conclusion of study)

Duration of follow-up: 3 months

Outcomes Primary: HbAlc, at baseline, at 1 month and 3 months

Secondary: PI, GI, PPD, CALs, and BOP were recorded at baseline, at 1 month and 3 months

Notes

Sample size calculation: Not reported

Data analysis: ITT

HbA1c assessment method: Not reported

SES: Not reported 
Kiran 2005 (Continued)

\author{
Adverse events: Not reported \\ Conflict of interests: Not reported \\ Clarification supplied by author \\ Note: teeth with periapical lesions were allocated additional treatment: \\ Gp A: 9 patients, 9 teeth: 4 extractions, 5 root canal treatment \\ Gp B: 5 patients, 5 teeth: 5 root canal treatment
}

\title{
Risk of bias
}

\begin{tabular}{|c|c|c|}
\hline Bias & Authors' judgement & Support for judgement \\
\hline $\begin{array}{l}\text { Random sequence genera- } \\
\text { tion (selection bias) }\end{array}$ & Low risk & $\begin{array}{l}\text { Quote: "A list was prepared in advance using random numbers. The list was } \\
\text { transferred to a series of sealed envelopes each containing the allocation on } \\
\text { the card" (from correspondence with a co-author) }\end{array}$ \\
\hline $\begin{array}{l}\text { Allocation concealment } \\
\text { (selection bias) }\end{array}$ & Low risk & $\begin{array}{l}\text { Quote: "The clinician opened the envelope in the series when the patient en- } \\
\text { tered the trial" (from correspondence with a co-author) }\end{array}$ \\
\hline Blinding of participants & High risk & Not possible \\
\hline $\begin{array}{l}\text { Blinding of clinical opera- } \\
\text { tor }\end{array}$ & High risk & Not possible \\
\hline $\begin{array}{l}\text { Incomplete outcome data } \\
\text { (attrition bias) } \\
\text { All outcomes }\end{array}$ & Low risk & $\begin{array}{l}\text { ITT analysis, although subjects who had surgical treatment were excluded } \\
\text { from statistical analysis. All participants underwent periodontal examination } \\
\text { at baseline and } 9 / 22 \text { and } 5 / 22 \text { had periapical lesions requiring treatment pri- } \\
\text { or to study start. Correspondence with co-author indicates: "HbA1c data was } \\
\text { recorded for all } 44 \text { trial participants, } 22 \text { for test and } 22 \text { for control patients. } \\
\text { There were no patients lost in the follow up period" }\end{array}$ \\
\hline $\begin{array}{l}\text { Selective reporting (re- } \\
\text { porting bias) }\end{array}$ & Unclear risk & All outcomes reported, except adverse events \\
\hline Other bias & Low risk & No other apparent biases \\
\hline
\end{tabular}

Koromantzos 2011

Methods Trial design: 2-arm, single-centre, parallel-design RCT

Location: Greece

Setting: Hospital

Number of centres: 1 , outpatient university diabetes clinic, Laiko Hospital, Athens

Recruitment period: January 2006 to December 2008

Funding source: European National Fund and National Resources (EPEAEK 2 PYTHAGORAS)

\begin{abstract}
Participants
Inclusion criteria: Diabetes Type: Type $2 \mathrm{DM}$ with HbA1c levels ranging from 7-10\%; moderate-to severe periodontitis; > 16 teeth present; PPD with at least 8 sites $\geq 6 \mathrm{~mm}$ and CAL $\geq 5 \mathrm{~mm}$ in at least 4 sites distributed to at least 2 quadrants
\end{abstract}


Exclusion criteria: Systemic antibiotic usage in last 3 months; non-surgical periodontal treatment during last 6 months; surgical periodontal treatment over last 12 months; current medication including usage of calcium channel blockers, phenytoin or cyclosporine; history of stroke or acute cardiovascular event over the past 12 months; renal dysfunction determined by creatinine levels $>1.5 \mathrm{mg} / \mathrm{dl}$ or liver dysfunction defined as AT/ALT levels >2.5 times ULN

Age at baseline: Overall: mean 59.52 yrs (SD 8.88); Gp A: mean 59.62 yrs (SD 7.95); Gp B: mean 59.42 yrs (SD 9.8)

\section{Sex (M:F): Overall M33:F27; Gp A M17:F13; Gp B M16:F14}

Tobacco use: Recorded at 3 levels - current, ex and non

Gp A: 4(13.3\%)/13(43.3\%)/13(43.3\%); Gp B: 7(23.3\%)/16(53.3\%)/7(23.3\%)

Alcohol consumption: Not recorded

Duration since diabetes diagnosis: Overall 7.8 yrs (SD 5.7); Gp A 7.76 yrs (SD 4.3); Gp B 7.84 yrs (SD 6.8)

Metabolic control: Fair mean HbA1c at baseline

Mean HbA1c at baseline: Gp A 7.87\% (SD 0.74); Gp B 7.59 (SD 0.66) (P value not reported)

Antidiabetic therapy: Insulin Gp A 12/30 (40\%), Gp B 7/30 (23.3\%) (P value not reported); OHA Gp A 21/30 (70\%), Gp B 27/30 (90\%) (P value not reported)

Mean BMI (kg/m²): Gp A 27.76 (SD) 3.68, Gp B 27.51 (SD) 3.83 (P value not reported)

Mean remaining teeth 23.52 (SD) 3.99, 24.23 (SD) 3.78 ( $P$ value not reported)

Other clinical investigations: Total cholesterol, total triglycerides, LDL-cholesterol, HDL-cholesterol

Number randomised: 60

Number evaluated: 60 (4 lost to follow-up in Gp A, 3 in Gp B)

Interventions Comparison: SRP + OHI versus supragingival cleaning + OHI

Gp A ( $n=30)$ : OHI (at baseline, 1 month and 3 months) + SRP (2 sessions, 1 week apart at baseline, using ultrasonic scaler and hand instruments, under local anaesthesia) + additional supportive SRP (at 1 month and 3 months) if required

$\mathrm{Gp} \mathrm{B} \mathrm{(} \mathrm{n}=30): \mathrm{OHI}$ (at baseline, 1 month and 3 months) + supragingival cleaning (described as "supragingival removal of all deposits (plaque and calculus) with an ultrasonic scaler." Delayed SRP provided to all after conclusion of study)

Duration of follow-up: 6 months

Outcomes Primary: HbAlc (recorded at baseline, 1 month, 3 and 6 months)

Secondary: CAL, PPD, BOP and GI (recorded at baseline, 1 month, 3 and 6 months) tween groups of $0.4 \%$ (90\% power, 2-sided type 1 error of $5 \%)$

HbA1c assessment method: High-performance liquid chromatography

\section{Data analysis: ITT}

SES: All Greek patients, no further details

Adverse events: Not reported

Conflict of interests: Authors declare no conflict of interests

Notes: Gp A: 2/30 had extractions at baseline 
Koromantzos 2011 (Continued)

Risk of bias

\begin{tabular}{lll}
\hline Bias & Authors' judgement & Support for judgement \\
\hline $\begin{array}{l}\text { Random sequence genera- } \\
\text { tion (selection bias) }\end{array}$ & Low risk & $\begin{array}{l}\text { Computer assignment undertaken by } 1 \text { author (PK) before recruitment. Se- } \\
\text { quential }\end{array}$ \\
& $\begin{array}{l}\text { Quote: "The randomization sequence was generated by one author (P.K.) be- } \\
\text { fore patient recruitment. Numbers from } 1 \text { to } 60 \text { were assigned to patients ac- } \\
\text { cording to their recruitment date (first recruited patient would be number } 1 \\
\text { and last would be number } 60) \text {. Random assignment into two groups of } 30 \text { pa- } \\
\text { tients each was then accomplished with the use of a computer program" }\end{array}$ \\
\hline
\end{tabular}

Allocation concealment Low risk $\quad 4$ containers numbered 1-60, designated for each visit of each patient maintain

(selection bias) masking

Quote: "Containers (numbered 1-60, four for each visit of each patient) were designated to maintain examiner blinding"

Blinding of participants High risk Not possible

Quote from correspondence with author: "Every patient after the screening examination was assigned to control or treatment groups according to their rank in that sequence (first that was recruited, 2nd, 3rd etc.). The participants did not know what category they were assigned in until they received SRP or prophylaxis, they were informed that they would have treatment at the beginning or at the end of the study"

\begin{tabular}{|c|c|c|}
\hline $\begin{array}{l}\text { Blinding of clinical opera- } \\
\text { tor }\end{array}$ & High risk & $\begin{array}{l}\text { Quote from correspondence with author: "The periodontist that performed } \\
\text { SRP or prophylaxis (same for all patients, P.K.) knew the allocation group of } \\
\text { the patients, right after the baseline visit" }\end{array}$ \\
\hline
\end{tabular}

Incomplete outcome data Low risk 100\% completion. ITT analysis
(attrition bias)

All outcomes

\begin{tabular}{|c|c|c|}
\hline \multirow[t]{2}{*}{$\begin{array}{l}\text { Selective reporting (re- } \\
\text { porting bias) }\end{array}$} & Unclear risk & $\begin{array}{l}\text { No change data for triglycerides, total cholesterol, LDL-cholesterol and HDL- } \\
\text { cholesterol. Adverse events not reported }\end{array}$ \\
\hline & & $\begin{array}{l}\text { Quote from correspondence with author: "..in our study we divided pock- } \\
\text { et depth and CAL in } 3 \text { categories, (percentage of shallow, medium and deep } \\
\text { pockets) and there is no available information in overall pocket depth or CAL." } \\
\text { Despite this, PPD and CAL data not considered to be a source of bias }\end{array}$ \\
\hline Other bias & Low risk & No other apparent biases \\
\hline
\end{tabular}

Kothiwale 2013

Methods

Trial design: 2-arm, single-centre, parallel-design $\mathrm{RCT}$

Location: India

Setting: Hospital

Number of centres: 1, Department of Periodontics, KLE VK Institute of Dental Sciences, Belgaum

Recruitment period: Unknown 
Kothiwale 2013 (Continued)

Funding source: Unknown

Participants

Inclusion criteria: Either sex; aged 25 or older; known cases of type 2 diabetes (minimum duration of 2 years); possessing >20 natural teeth; and receiving oral hypoglycaemic medications

Exclusion criteria: History of smoking, haemoglobinopathies, or hypertension; receiving insulin therapy, renal dialysis or requiring hospitalisation; undergone periodontal therapy in prior 6 months; antibiotic/anti-inflammatory drugs taken in prior 3 months; have abnormal hepatic function; pregnant or lactating

Age at baseline: Gp A: mean 57.7 yrs (SD 8.61); Gp B: mean 56.4 yrs (SD 11.53)

Sex (M:F): Overall: M32:F18; Gp A: M15:F10; Gp B: M17:F8

Tobacco use: Excluded from participation if possess history of smoking

Weight: Not reported

BMI: Gp A: 23.7 (SD 1.92); Gp B: 23.85 (SD 1.65)

Alcohol consumption: Not reported

Diabetes type: All T2 DM

Duration since diabetes diagnosis: Gp A: mean 5.3 yrs (SD 2.76); Gp B: 5.2 yrs (SD 2.20)

Metabolic control: Fair mean $\mathrm{HbA1c}$ at baseline

Mean HbA1c at baseline: Gp A: 8.16 (SD 0.61); Gp B: 7.94 (SD 0.66)

Antidiabetic therapy: All in receipt of oral hypoglycaemic medication

Quote: "The oral hypoglycemic drugs for diabetes, diet and physical therapy was unchanged throughout the course of the study as monitored by the physician"

Other investigations: Change in periodontal status (by CPI and LOA scores)

Other medical conditions: Not reported

Number randomised: $50(\mathrm{Gp} \mathrm{A} n=25 ; \mathrm{Gp} \mathrm{B} n=25)$

Number evaluated: Not reported

\section{Comparison: SRP + OHI versus no treatment}

Gp A ( $n=25)$ : SRP after baseline examination (by ultrasonic scaler, hand scaler and curette across varying numbers of sessions - dependent of treatment needs of individual patients), followed a further SRP session (unspecified time point) by same investigator, and provision of $\mathrm{OHI}$

Gp B ( $n=25)$ : No treatment (followed by SRP and OHI after end of study)

Duration of follow-up: 3 months

\section{Outcomes}

Primary: Change in $\mathrm{HbA} 1 \mathrm{c}$ from baseline to 3 months

Secondary: None

Data analysis: Per-protocol

SES: Education status data provided:

Overall: Illiterate $n=11(22 \%) ;$ primary school $n=14(28 \%)$; high school $n=15(30 \%)$; graduate $n=10$ $(20 \%)$

Gp A: Illiterate $n=5(20 \%)$; primary school $n=10(40 \%)$; high school $n=6(24 \%)$; graduate $n=4(16 \%)$ Gp B: Illiterate $n=6(24 \%)$; primary school $n=4(16 \%)$; high school $n=9(36 \%)$; graduate $n=6(24 \%)$ 
Kothiwale 2013 (Continued)

\section{Adverse events: Not reported}

HbA1c assessment method: High-pressure liquid chromatography (HPLC)

Conflict of interests: Not reported

\section{Risk of bias}

\begin{tabular}{|c|c|c|}
\hline Bias & Authors' judgement & Support for judgement \\
\hline $\begin{array}{l}\text { Random sequence genera- } \\
\text { tion (selection bias) }\end{array}$ & Unclear risk & $\begin{array}{l}\text { Simply states } 50 \text { patients randomly assigned into } 2 \text { groups. No indication of } \\
\text { method }\end{array}$ \\
\hline $\begin{array}{l}\text { Allocation concealment } \\
\text { (selection bias) }\end{array}$ & Unclear risk & Not reported \\
\hline Blinding of participants & High risk & Not possible \\
\hline $\begin{array}{l}\text { Blinding of clinical opera- } \\
\text { tor }\end{array}$ & High risk & $\begin{array}{l}\text { Not reported, but assumed not possible as only intervention group patients } \\
\text { would have received care }\end{array}$ \\
\hline \multirow[t]{2}{*}{$\begin{array}{l}\text { Incomplete outcome data } \\
\text { (attrition bias) } \\
\text { All outcomes }\end{array}$} & High risk & $\begin{array}{l}\text { No patient flow provided or any drop-outs indicated, although states "After } \\
\text { the non-surgical therapy was completed, patients were revaluated for surgical } \\
\text { treatment needs. The data concerning the group of patients who had surgical } \\
\text { treatment were excluded in the statistical analysis" }\end{array}$ \\
\hline & & $\begin{array}{l}\text { Per-protocol analysis: not all participants analysed in groups randomised to, } \\
\text { regardless of intervention actually received }\end{array}$ \\
\hline $\begin{array}{l}\text { Selective reporting (re- } \\
\text { porting bias) }\end{array}$ & Unclear risk & Planned outcomes reported on; however no reporting of adverse events \\
\hline Other bias & Unclear risk & $\begin{array}{l}\text { Unpublished data, and therefore without peer-review. Author indicated inten- } \\
\text { tion to publish study in full in near future }\end{array}$ \\
\hline
\end{tabular}

Li 2011

Methods Trial design: 3 -arm, multicentre, parallel-design RCT

Location: Peking, China

Setting: Community

Number of centres: 6 community healthcare centres

Recruitment period: Not reported

Funding source: National Key Project of Science and Technical Supporting Programs of China, National Natural Science Foundation of China, "211" Project Foundation, Mega-projects of Science Research for the $10^{\text {th }}$ Five-year Plan

Participants

Inclusion criteria: Type 2 DM (the diagnostic criteria was 1999 WHO DM diagnostic criteria) with chronic periodontitis (at least 1 tooth with $P D \geq 3 \mathrm{~mm}$ and $A L \geq 3 \mathrm{~mm}$ ). The number of residual teeth must have exceeded 16 and no receipt of any periodontal treatment within 1 year

Exclusion criteria: Aggressive periodontitis, severe chronic or debilitating disease; long-term usage of antibiotics or steroids

Age at baseline: Gp A: 60.86 yrs (SD 10.22); Gp B: 64.21 yrs (SD 5.99); Gp C: 61.64 yrs (SD 9.6) 
Li 2011 (Continued)

Sex (M:F): Overall M28:F38; Gp A M9:F13; Gp B M8:F11;Gp C M11:F14

Tobacco use: Gp A (9.1\%); Gp B (15.8\%); Gp C (12\%)

Alcohol consumption: Not reported

Diabetes type (I/II): Gp A (0/22); Gp B (0/19); Gp C (0/25)

Duration since diabetes diagnosis: Gp A 6.5 (SD) 5.1 yrs; Gp B 8.84 (SD) 5.77 yrs; Gp C 7.92 (SD) 5.14 yrs

Metabolic control: Fair mean HbA1c at baseline

Mean HbA1c at baseline: Gp A: 7.64 (SD 1.77); Gp B: 8.15 (SD 1.97); Gp C: 8.12 (SD 1.88)

Antidiabetic therapy: Gp A (oral hypoglycaemic agents: $77.3 \%$ /insulin injection: $27.3 \%$ ); Gp B

(78.9\%/21.1\%); Gp C (76\%/16\%)

Other clinical investigations: FBG (fasting blood glucose); modified bleeding index

Other medical conditions: Diabetes complications Gp A (27.3\%); Gp B (21.1\%); Gp C (32\%)

Number randomised: 66

Number evaluated: Not reported

Interventions

Comparison: Non-surgical periodontal treatment versus supragingival scaling versus no intervention

Gp A $(n=22)$ : Periodontal initial therapy: periodontal non-surgical treatment given by periodontists (details not given)

Gp B ( $n=19)$ : Professional mechanical tooth cleaning: coronal/supragingival scaling given by oral hygienists (details not given)

Gp C ( $n=25)$ : Non-clinical therapy: no active intervention

Duration of follow-up: 6 months

Outcomes

Primary: HbA1c (at baseline, 6 weeks, 3 and 6 months)

Secondary: Probing depth, attachment loss, plaque index - change data only for periodontal parameters

Notes

Sample size calculation: Not reported

Data analysis: Assumed ITT

SES: Not reported

Adverse events: Unknown, was a stated secondary outcome in paper

HbA1c assessment method: Not reported

Conflict of interests: Not reported

Translation by Chunjie Li, May 2014

\section{Risk of bias}

\begin{tabular}{lll}
\hline Bias & Authors' judgement & Support for judgement \\
\hline $\begin{array}{l}\text { Random sequence genera- } \\
\text { tion (selection bias) }\end{array}$ & Unclear risk & No information \\
\hline
\end{tabular}


Li 2011 (Continued)

\begin{tabular}{lll}
$\begin{array}{l}\text { Allocation concealment } \\
\text { (selection bias) }\end{array}$ & Unclear risk & No information \\
\hline Blinding of participants & Unclear risk & No information \\
\hline $\begin{array}{l}\text { Blinding of clinical opera- } \\
\text { tor }\end{array}$ & Unclear risk & No information \\
\hline $\begin{array}{l}\text { Incomplete outcome data } \\
\text { (attrition bias) } \\
\text { All outcomes }\end{array}$ & Unclear risk & No information \\
\hline $\begin{array}{l}\text { Selective reporting (re- } \\
\text { porting bias) }\end{array}$ & Unclear risk & No information \\
\hline $\begin{array}{l}\text { Other bias } \\
\text { Unclear risk }\end{array}$ & $\begin{array}{l}\text { No way to verify if other biases exist due to translation of data extraction com- } \\
\text { ponents }\end{array}$
\end{tabular}

Llambés 2008

Methods

Trial design: 2-arm, single-centre, parallel-design RCT

Location: Spain

Setting: Hospital

Number of centres: 1, Dr Peset University Hospital, Valencia, Spain.

Recruitment period: September 2003 to March 2004

Funding source: Not reported

Participants

Inclusion criteria: Diabetes for more than 1 year, and none of them had other major illnesses or severe diabetic complications; patients had not taken antibiotics for at least 3 months prior to baseline and did not have any active infection; a panoramic radiograph was taken to assure that neither extensive caries nor periapical lesions were present; eligible subjects had 14 or more natural teeth, of which at least 5 had a site with PPD $\geq 5 \mathrm{~mm}$ and $\mathrm{CAL} \geq 3 \mathrm{~mm}$ i.e. moderate to severe periodontal disease

Exclusion criteria: Periodontal treatment or professional cleaning of the teeth in year prior to the study; pregnant and breastfeeding women

Age at baseline: Overall mean 35.3 yrs (SD 9.0); Gp A mean 36.8 yrs (SD 9.5); Gp B mean 33.8 yrs (SD 9)

Sex (M:F): Overall M30:F30; Gp A M17:F13; Gp B M13:F17

Tobacco use: Overall $n=22 ; G p A n=11 ; G p ~ B n=11$

Alcohol consumption: Not reported

Diabetes type: Type $1 \mathrm{DM}$

Duration since diabetes diagnosis: At least 1 year, Gp A 14 (SD 7.5), Gp B 15 (10) years

Metabolic control: Fair mean HbA1c at baseline

Mean HbA1c at baseline: Gp A: 7.64 (SD 1.81); Gp B: 7.51 (SD 1.36)

Antidiabetic therapy: All in receipt of insulin

Other clinical investigations: Fructosamine 
Llambés 2008 (Continued)

\section{Number randomised: 72}

Number evaluated: 60

\section{Interventions \\ Comparison: SRP + OHI + chlorhexidine rinse + systemic doxycycline versus SRP + OHI + chlorhex- idine rinse}

Gp A ( $\mathrm{n}=30$ ): $\mathrm{OHI}$ (instruction on Bass brushing technique and interproximal cleaning) + SRP (under local anaesthesia, in 1 or 2 sessions, 1 week apart depending on periodontal disease severity and number of natural teeth) + chlorhexidine rinse (20 ml for $30 \mathrm{sec}$, twice daily for 12 weeks) + systemic doxycycline (200 $\mathrm{mg}$ on day 1 , then $100 \mathrm{mg} /$ day for 14 days)

Gp B ( $\mathrm{n}=30)$ : As above, without systemic doxycycline

Duration of follow-up: 3 months

Outcomes Primary: Changes in HbAlc

Secondary: PI, BOP, PPD, CAL (recorded but not presented, and not provided by authors)

Notes

Sample size calculation: Reported that "enough statistical power to detect $\mathrm{HbA} 1 \mathrm{c}$ changes $\geq 0.3 \%$ with a risk of $0.05 . "$ Possibly a post hoc calculation

Compliance with chlorhexidine rinsing reported to be same in both groups

Data analysis: Per-protocol

HbA1c assessment method: Not reported

SES: Not reported

Adverse events: Not reported

Conflict of interests: Not reported

\section{Risk of bias}

\begin{tabular}{lll}
\hline Bias & Authors' judgement & Support for judgement \\
\hline $\begin{array}{l}\text { Random sequence genera- } \\
\text { tion (selection bias) }\end{array}$ & Unclear risk & $\begin{array}{l}\text { Quote: "The sample was randomized, allowing the subjects to self-select a } \\
\text { coded number contained in an envelope; this number identified the group to } \\
\text { which the patient was assigned (group 1 or 2)" } \\
\text { Comment: Method of code generation not stated }\end{array}$ \\
\hline $\begin{array}{l}\text { Allocation concealment } \\
\text { (selection bias) }\end{array}$ & Unclear risk & Comment: Unclear if envelope was sealed and opaque \\
\hline Blinding of participants & High risk & No placebo tablets given \\
\hline $\begin{array}{l}\text { Blinding of clinical opera- } \\
\text { tor }\end{array}$ & Unclear risk & Not reported \\
\hline
\end{tabular}

Incomplete outcome data High risk

(attrition bias)

All outcomes
Quote: "At the end of the study, 12 subjects were dropped out because they did not follow appropriately the study protocol or due to active acute infections during posttreatment period"

Per-protocol analysis: not all participants analysed in groups randomised to, regardless of intervention actually received 
Llambés 2008 (Continued)

Selective reporting (re- High risk PI, BOP, PPD, CAL recorded but not reported. Adverse events also not reported porting bias)

Other bias Low risk No other apparent biases

Macedo 2014

Methods

Trial design: 2 -arm, single-centre, parallel-design RCT

Location: Brazil

Setting: Not reported

Number of centres: 1 , School of Dentistry of Sao Paulo University

Recruitment period: Not stated

Funding source: Financial support from the State of Sao Paulo Research Foundation (FAPESP protocol number 06/04600-9)

Participants

Inclusion criteria: Type 2 diabetes diagnosed for $>5$ years, $\mathrm{HbA} 1 \mathrm{c}>7 \%, \geq 1$ site with $\mathrm{PPD} \geq 5 \mathrm{~mm}$ on each quadrant, 2 teeth with $\geq 6 \mathrm{~mm} \mathrm{CAL}$

Exclusion criteria: Use of antibiotics or periodontal treatment in the previous 6 months; smoking within the past 5 years; pregnancy or lactation; major diabetic complication; concomitant medical therapy to systemic complications

Age at baseline: 48.73 yrs (SD 7.11); Gp A: 48.1 yrs (SD 9.0); Gp B: 49.4 yrs (SD 6.8)

Sex (M:F): overall M11:F19; Gp A: M6:F9; Gp B: M5:F10

Tobacco use: Specified as exclusion criteria to have smoked in prior 5 years

Alcohol consumption: Not reported

Diabetes type: All T2 DM

Duration since diabetes diagnosis: Not specifically reported, although all participants diagnosed for over 5 years

Metabolic control: Fair mean HbA1c at baseline

Mean HbA1c at baseline: Gp A 7.64 (SD 1.81); Gp B: 7.51 (SD 1.36)

Quote: "poorly controlled diabetes with an elevated mean HbA1c serum level of 8.33\%"

Antidiabetic therapy: Not reported

Other medical conditions: Not reported

Other clinical investigations: Suppuration

Number randomised: 30

Number evaluated: 30

Interventions

SRP + adjunctive antimicrobial + aPDT + OHI (x 7) versus SRP + adjunctive antimicrobial + OHI (x 7)

Gp A ( $n=15)$ : SRP (using hand instruments, ultrasonic instrumentation, and local anaesthesia in 2-4 sessions within 24-36 hours by the same operator) + adjunctive doxycycline (100 mg p/d x 2 weeks, initiated 1 day prior to SRP commencing) + phenothiazine chloride photo sensitiser-induced antimicrobial photodynamic therapy (aPDT: single-episode of diode laser using $660 \mathrm{~nm}$ wavelength, with $10 \mathrm{mg}$ / 
$\mathrm{ml}$ concentration photo sensitiser, continuously deposited in each pocket for 1 minute per tooth, followed by distilled water irrigation to remove excess). $\mathrm{OHI}$ delivered 14 days prior to treatment

Gp B ( $n=15)$ : SRP (using hand instruments, ultrasonic instrumentation, and local anaesthesia in 2-4 sessions within 24-36 hours by the same operator) + adjunctive doxycycline (100 mg p/d x 2 weeks, initiated 1 day prior to SRP commencing). OHI delivered 14 days prior to treatment

Note: All patients received supragingival professional tooth cleaning 7 days prior to treatment, $\mathrm{OHI}$ review every 14 days, and prophylaxis for 3 months

\begin{tabular}{ll}
\hline Outcomes & Primary: HbAlc at baseline and 3 months \\
& Secondary: PPD, CAL, BOP, and PI at baseline and 3 months \\
& Duration of follow-up: 3 months \\
\hline Notes & Sample size calculation: 30 participants required (76\% power, .05 significance) \\
& Data analysis: ITT \\
& $\begin{array}{l}\text { Adverse events: Quote: "Healing was uneventful on all cases. No adverse effects were reported by any } \\
\text { of the subjects" }\end{array}$
\end{tabular}

HbA1c assessment method: Automated immunoturbidimetric method using $15 \mathrm{ml}$ samples

SES: Not reported

Conflict of interests: Not reported

\section{Risk of bias}

\begin{tabular}{lll}
\hline Bias & Authors' judgement & Support for judgement \\
\hline $\begin{array}{l}\text { Random sequence genera- } \\
\text { tion (selection bias) }\end{array}$ & Low risk & $\begin{array}{l}\text { Quote: "A randomization approach using computer-generated random num- } \\
\text { bers was employed to assign subjects to one of the following two treatment } \\
\text { modalities" }\end{array}$ \\
\hline $\begin{array}{l}\text { Allocation concealment } \\
\text { (selection bias) }\end{array}$ & Unclear risk & Not reported \\
\hline $\begin{array}{l}\text { Blinding of participants } \\
\text { Blinding of clinical opera- }\end{array}$ & Unclear risk & Not reported \\
\hline $\begin{array}{l}\text { tor } \\
\text { (attrition bias) } \\
\text { All outcomes }\end{array}$ & Not reported \\
\hline $\begin{array}{l}\text { Selective reporting (re- } \\
\text { porting bias) }\end{array}$ & Low risk & No drop-outs, all 30 completed the trial. ITT analysis \\
\hline \begin{tabular}{l} 
Other bias \\
\hline
\end{tabular} & Low risk & All outcomes reported, including adverse events \\
\hline
\end{tabular}

Madden 2008

Methods Trial design: 2-arm, parallel-design RCT

Location: USA 


\section{Setting: Not reported \\ Number of centres: Not reported \\ Recruitment period: Not reported}

Funding source: Financial support from the Medical Research Foundation of Oregon (Oregon Health and Science University)

Participants

Inclusion criteria: Mild/moderate gingivitis or periodontitis; $\mathrm{HbA} 1 \mathrm{c}>7.0 \%-<13.11 \%$; elevated $\mathrm{HbAlc}>1$ year; >15 natural teeth; $18-80$ years old

Exclusion criteria: Periodontal surgery/antibiotic prophylaxis required; conditions/medications interfering with diabetic control; antibiotic/steroid/hydantoin/NSAID use; immunosuppression; rheumatoid arthritis; HIV; pregnancy; tobacco use; fixed orthodontic appliances; inability to give consent; unable/unwilling to remain enrolled for 8 months

Age at baseline: Not reported

Sex (M:F): Not reported specifically, only "treatment groups were balanced for gender only"

Tobacco use: Specified as exclusion criteria

Alcohol consumption: Not reported

Diabetes type: All T2 DM

Duration since diabetes diagnosis: Not reported

Metabolic control: Fair-poor mean $\mathrm{HbA1c}$ at baseline

Mean $\mathrm{HbA1C}$ at baseline presented by 4 subgroups, without SDs, and only for the 42 completing patients:

Gp A: No medication change: severe Gp: $10.3 \%(n=6)$ mild Gp: $8.0 \%(n=6)$; medication change: severe Gp: $11.10 \%(n=5)$ mild Gp: $7.8 \%(n=4)$

Gp B: No medication change: severe Gp: $10.0 \%(n=5)$ mild Gp: $8.1 \%(n=10)$; medication change: severe Gp: $10.2 \%(n=2)$ mild Gp: $7.7 \%(n=4)$

Antidiabetic therapy: Not reported

Other medical conditions: Not reported

Other clinical investigations: Calculus index

Number randomised: 50

Number evaluated: 42 (Gp A $n=21 ; G p B n=21)$

Losses: Died (unrelated to study) $n=2$; withdrawn for inclusion/exclusion violations (smoking indicated as example) $n=6$

Gp A ( $n=25)$ : SRP x 5 ("inflamed periodontal pockets with clinical attachment loss" only; single session (60-90 $\mathrm{min}$ ) delivery by the same operator) + adjunctive $0.12 \%$ chlorhexidine gluconate rinse (Peridex, Zila Pharmaceuticals: 30 seconds oral rinsing bid for duration of study) $+\mathrm{OHI}$. All delivered at baseline, 2, 4, 6 and 8 months follow-up

Gp B ( $n=25)$ : SRP $\times 2$ (inflamed periodontal pockets with clinical attachment loss only; single session $(60-90 \mathrm{~min})$ delivery by the same operator $)+\mathrm{OHI} \times 2$. Both delivered at baseline and 6-month follow-up

\section{Outcomes}

Secondary: PPD, CAL (indicated as CEJ in graph), GI, and PI at baseline, 6 and 8 months 
Madden 2008 (Continued)

Duration of follow-up: 8 months

Notes Sample size calculation: Indication calculation occurred, although not specifically reported. Quote: "Sample size presented the major flaw in this study and was exacerbated by the need to accommodate statistically for 15 subjects [Gp A: $n=9 ; G p$ B: $n=6$ ] undergoing changes in their diabetes medications"

Data analysis: Not reported

Adverse events: Not reported

HbAlc assessment method: Not reported

SES: Not reported

Conflict of interests: Not reported

\begin{tabular}{|c|c|c|}
\hline \multicolumn{3}{|l|}{ Risk of bias } \\
\hline Bias & Authors' judgement & Support for judgement \\
\hline $\begin{array}{l}\text { Random sequence genera- } \\
\text { tion (selection bias) }\end{array}$ & Low risk & Quote: "consenting subjects were randomized (by flip of coin)" \\
\hline $\begin{array}{l}\text { Allocation concealment } \\
\text { (selection bias) }\end{array}$ & Unclear risk & Not reported \\
\hline Blinding of participants & High risk & Not possible \\
\hline $\begin{array}{l}\text { Blinding of clinical opera- } \\
\text { tor }\end{array}$ & Unclear risk & $\begin{array}{l}\text { Not specifically reported, and probably not possible as all treatment delivered } \\
\text { by single operator. Quote: "All treatment provided in this protocol was deliv- } \\
\text { ered by the research dental hygienist" }\end{array}$ \\
\hline $\begin{array}{l}\text { Incomplete outcome data } \\
\text { (attrition bias) } \\
\text { All outcomes }\end{array}$ & High risk & $\begin{array}{l}\text { Per-protocol analysis: not all participants analysed in groups randomised to, } \\
\text { regardless of intervention actually received } \\
\text { Rationale provided for all missing patients, although not by group }\end{array}$ \\
\hline $\begin{array}{l}\text { Selective reporting (re- } \\
\text { porting bias) }\end{array}$ & High risk & $\begin{array}{l}\text { Primary outcome data presented as subgroups (severe/mild HbAlc) and ta- } \\
\text { bles split by patients not requiring a change in antidiabetic therapy. All data } \\
\text { presented without SDs. Adverse events also not reported. Clarification request } \\
\text { email to authors bounced }\end{array}$ \\
\hline Other bias & Unclear risk & $\begin{array}{l}\text { No patient characteristics data presented. Potential baseline incompatibility } \\
\text { between groups. Inclusion criteria states "gingivitis or periodontitis" without } \\
\text { indication of proportion characteristics. Concern study population is not nec- } \\
\text { essarily equal to other included studies due to potential inclusion of gingivitis }\end{array}$ \\
\hline
\end{tabular}

Miranda 2014

Methods

Trial design: 2 -arm, single-centre, parallel-design RCT

Location: Brazil

Setting: Hospital

Number of centres: 1, Periodontal Clinic of Guarulhos University, São Paulo

Recruitment period: Assumed 1 month, September-October 2011, as 12 month-long follow-up period. Quote: "The study was conducted between September 2011 and October 2012" 
Funding source: São Paulo State Research Foundation (São Paulo, São Paulo, Brazil, \# 2011/14872-4; 2013/01072-5)

\section{Participants} Inclusion criteria: $>35$ yrs; diagnosed type $2 \mathrm{DM}>5 \mathrm{yrs}$; receiving hypoglycaemic treatment/supplementation; $\mathrm{HbA1c}$ level $>6.5 \%-<11 \%$; at least 15 teeth (excluding 3rd molars, or decayed teeth requiring extraction); $>30 \%$ of sites with $>4 \mathrm{~mm} \mathrm{PD/CAL}$; minimum 6 teeth with at least 1 site $\mathrm{BOP}$ and $>5 \mathrm{~mm} \mathrm{PD/}$ CAL

Exclusion criteria: Smoker within prior 5 years; SRP in prior 12 months; antimicrobial therapies in prior 6 months; antimicrobial-containing mouthrinse use in prior 3 months; pregnancy; lactation; allergic to metronidazole or amoxicillin; systemic condition affecting periodontitis progression (including immunological disorders, osteoporosis); conditions requiring prophylactic antibiotics; orthodontic appliances; extensive prosthetic rehabilitation; major complications of DM

Age at baseline: Overall: mean 53.9 yrs (SD 8.1); Gp A: 54.0 yrs (SD 8.2); Gp B: 53.7 yrs (SD 8.0)

Sex (M:F): Overall M30:F26; Gp A: M12:F17; Gp B: M18:F9

Tobacco use: Specified as exclusion criteria to have smoked in prior 5 years

Alcohol consumption: Not reported

Diabetes type: All type 2

Duration since diabetes diagnosis: Overall 7.7 yrs (SD 3.4); Gp A: 8.0 yrs (SD 3.2); Gp B: 7.4 yrs (SD 3.6)

Metabolic control: Poor mean $\mathrm{HbA1C}$ at baseline

Mean HbA1c at baseline: Gp A: 8.53 (SD 1.56); Gp B: 8.99 (SD 1.63)

Antidiabetic therapy: Required by inclusion criteria. Quote: "all subjects included in this study reported to be under metformin or glibenclamide treatment. In addition, two subjects per group also reported to be under insulin supplementation"

Other medical conditions: Not reported

Other investigations: Suppuration; differences between groups for disease progression (low, moderate, high risk patients); FPG

Number randomised: 58 (Gp A: $n=29 ; G p$ B: $n=29)$

Number evaluated: 56 (Gp A: $n=29 ; G p$ B: $n=27$ - 2 did not attend baseline visit) timicrobial

Gp A ( $n=29)$ : SRP (4-6 1 hour-sessions within 14 days of baseline, under local anaesthesia, using manual curettes and ultrasonic device); $\mathrm{OHI}$ (brushing technique and provision of toothpaste); and metronidazole ( $3 \times 400 \mathrm{mg} \mathrm{p} / \mathrm{d}$ for 14 days) and amoxicillin ( $3 \times 500 \mathrm{mg} \mathrm{p} / \mathrm{d}$ for 14 days) administered immediately after first SRP session

Gp B ( $n=29)$ : SRP (4-6 1 hour-sessions within 14 days of baseline, under local anaesthesia, using manual curettes and ultrasonic device); $\mathrm{OHI}$ (brushing technique and provision of toothpaste); and placebo pills (6 p/d for 14 days) administered immediately after first SRP session

Duration of follow-up: 12 months 
Adverse events: $\mathrm{Gp} \mathrm{A}: \mathrm{n}=14(48 \%)$; Gp B: $\mathrm{n}=6(22 \%)$ report at least 1 adverse event of antibiotic/placebo use

Gp A: Diarrhea $n=7(24 \%)$; headache $n=4(14 \%) ;$ metallic taste $n=4(14 \%)$; nausea/vomiting $n=5$ $(17 \%)$

Gp B: Diarrhea $n=3(11 \%)$; headache $n=1(4 \%)$; metallic taste $n=2(7 \%)$; nausea/vomiting $n=2(7 \%)$

Quote: "All subjects stated that the medications did not cause any major disturbance in their daily routine and, therefore, they would repeat the antibiotic treatment, if necessary"

HbA1c assessment method: High-performance liquid chromatography

Conflicts of interest: Authors declare no conflict of interest. All participants received Colgate Total toothpaste (attributed to Colgate Palmolive Co., São Paulo, SP, Brazil) for use throughout trial, but it is not clear if products were donated by the company

Trial ID: NCT02135952

\section{Risk of bias}

\begin{tabular}{lll}
\hline Bias & Authors' judgement & Support for judgement \\
\hline $\begin{array}{l}\text { Random sequence genera- } \\
\text { tion (selection bias) }\end{array}$ & Low risk & $\begin{array}{l}\text { Quote: "..each selected subject received a code number and one of the study } \\
\text { coordinators (M.Fe.) used a computer-generated table to randomly allocate } \\
\text { them into one of the }[. . .] \text { treatment groups" }\end{array}$ \\
& $\begin{array}{l}\text { "..randomization was stratified to allow each operator to treat half of the sub- } \\
\text { jects in each treatment group" }\end{array}$
\end{tabular}

\begin{tabular}{ll}
\hline $\begin{array}{l}\text { Allocation concealment } \\
\text { (selection bias) }\end{array}$ & Quote: "The same pharmacy (Proderma Farmacia de Manipulacao Ltda, Piraci- \\
& caba, SP, Brazil) prepared the antibiotics and placebos. Identical plastic bot- \\
& tles containing the antibiotics or placebos were sent to one of the study coor- \\
& dinators (P.M.D.), who marked the code number of each subject on each bot- \\
te, according to the therapy assigned. Allocation concealment was assured & by means of sequentially numbered drug containers of identical appearance. \\
& Study personnel, including the examiner (T.S.M.), the two operators, the in- \\
vestigator responsible for the data analysis (M.Fa.) and the participants were & blinded to treatment assignment. Code breaking was performed after final sta- \\
tistical analysis"
\end{tabular}

\begin{tabular}{ll}
\hline Blinding of participants $\quad$ Low risk & $\begin{array}{l}\text { Quote: "..participants were blinded to treatment assignment. Code breaking } \\
\text { was performed after final statistical analysis" }\end{array}$
\end{tabular}
was performed after final statistical analysis"

\begin{tabular}{|c|c|c|}
\hline $\begin{array}{l}\text { Blinding of clinical opera- } \\
\text { tor }\end{array}$ & Low risk & $\begin{array}{l}\text { Quote: "Study personnel, including [...], the two operators, [...] were blinded } \\
\text { to treatment assignment. Code breaking was performed after final statistical } \\
\text { analysis" }\end{array}$ \\
\hline
\end{tabular}

Incomplete outcome data Low risk (attrition bias) being uncontactable, however still included in study's ITT analysis

All outcomes
Comment: Both arms' attrition occurred at 6-month follow-up, due to patients Quote: "Four subjects in the control group and two in the test group were lost during the follow up visits"
"The data were evaluated using intention-to-treat analysis with last observa- tion carried forward"
All outcomes reported, although PD/CAL provided as subsets (moderate (4-6 $\mathrm{mm}$; deep $>7 \mathrm{~mm}$ ) thus not usable in meta-analysis Despite this, not consid- ered to be a source of bias


Miranda 2014 (Continued)
Other bias
Low risk
No other apparent biases

Moeintaghavi 2012

Trial design: 2-arm, single-centre, parallel-design RCT
Location: Iran
Setting: Hospital
Number of centres: 1, Periodontics Department, Mashhad Dental School
Recruitment period: June 2007 to September 2008 (Parsian Diabetes Clinic and Mashhad Diabetics
Centre)
Funding source: Grant from Mashhad University of Medical Sciences

Participants

Inclusion criteria: Mild-moderate periodontitis (AAP criteria); diagnosis of T2 DM with $\mathrm{HbA} 1 \mathrm{C}>7 \%$; no major complications of diabetes; controlled by $\mathrm{OHA}$ (glybenglamide and metformin) but not insulin administration; no periodontal treatment or antibiotic administration in last 6 months

Exclusion criteria: Presence of systemic diseases other than T2 DM that could influence course of periodontal disease; intake of immunosuppressive drugs, steroids, hydantoin, or NSAIDs; tobacco use; pregnancy or intention to become pregnant during study period; fixed orthodontic appliances; refusal or inability to give informed consent

Age at baseline: Overall: 50.29 yrs (SD 3); M 52.48 yrs (SD 3); F 48.1 yrs (SD 3) (by sex $\mathrm{P}=0.9$ )

No detail of age by group allocation

Sex (M:F): Overall M20:F20; Gp A M9:F13; Gp B M11:F7 (P=0.341)

Tobacco use: Excluded

Alcohol consumption: Not reported, although consumption of alcohol is illegal in Iran

Diabetes type: $\mathrm{T} 2 \mathrm{DM}$

Duration since diabetes diagnosis: Not reported

Metabolic control: Poor mean $\mathrm{HbAlc}$ at baseline

Mean HbA1c at baseline: Gp A 8.15\% (SD 2.22); Gp B 8.72\% (SD 1.82) (P= 0.304)

Antidiabetic therapy: All in receipt of oral hypoglycaemic medication (no insulin)

Other clinical investigations: Biochemical markers TG, TC (total cholesterol), LDL, HDL, FPG

Number randomised: 40

Number evaluated: 40

Interventions

\section{Comparison: SRP versus no intervention}

Gp A ( $n=22)$ : SRP (ultrasonic device, standard periodontal curettes, local anaesthetic and no limitation on time)

Gp B ( $n=18)$ : No treatment (delayed SRP provided after completion of trial)

Duration of follow-up: 3 months 
Secondary: CAL, PPD, PI and GI (at baseline and 3 months)

Notes HbA1c assessment method: Cobas Integra 700; Roche Diagnostics, Germany

Data analysis: ITT

Conflict of interests: Not reported

Adverse events: Not reported

SES: Not reported

Sample size calculation: A priori calculation based on Kiran 2005 and Rodrigues 2003 of 20 per group $(\alpha=0.05$ and $\beta=0.2)$

Trial ID: NCT01252082

\section{Risk of bias}

\begin{tabular}{|c|c|c|}
\hline Bias & Authors' judgement & Support for judgement \\
\hline $\begin{array}{l}\text { Random sequence genera- } \\
\text { tion (selection bias) }\end{array}$ & Low risk & $\begin{array}{l}\text { Quote: "Patients were randomly divided into treatment and non-treatment } \\
\text { (control) groups by the study research assistant (KK) using a computer gener- } \\
\text { ated random numbers table" }\end{array}$ \\
\hline $\begin{array}{l}\text { Allocation concealment } \\
\text { (selection bias) }\end{array}$ & Unclear risk & $\begin{array}{l}\text { Examiner (AMT) at baseline "blinded to subjects' group assignment."Although } \\
\text { 'AMT' blinded, randomisation statement relates to 'KK' and therefore unclear if } \\
\text { allocation concealment occurred }\end{array}$ \\
\hline Blinding of participants & High risk & Not possible \\
\hline $\begin{array}{l}\text { Blinding of clinical opera- } \\
\text { tor }\end{array}$ & High risk & Not possible \\
\hline $\begin{array}{l}\text { Incomplete outcome data } \\
\text { (attrition bias) } \\
\text { All outcomes }\end{array}$ & Low risk & $\begin{array}{l}\text { ITT analysis. All patients completed the study, however several non-planned } \\
\text { treatments occurred: Reported extractions - } 1 \text { per group } \\
\text { Endodontic treatment to } 1 \text { in Gp A }\end{array}$ \\
\hline $\begin{array}{l}\text { Selective reporting (re- } \\
\text { porting bias) }\end{array}$ & Unclear risk & $\begin{array}{l}\text { Age differences not reported between group but by sex instead } \\
\text { Adverse events not reported }\end{array}$ \\
\hline Other bias & Low risk & No other apparent biases \\
\hline
\end{tabular}

Recruitment period: December 2008 -? 
NCT00801164 (Continued)

Funding source: Biomedical Development Corporation (manufacturer of an iodine mouthrinse product: www.biodevcorp.com/products/icleanmouths/); Methodist Healthcare Ministries of South Texas; Foundation Of Collaborative Unique Science (FOCUS)

Participants

Inclusion criteria: Either sex; over 35 years old; self reported T2 DM ( $>3$ months duration); HbA1c value between $7.0 \%$ and $12 \%$; no change in diabetes-related medications during prior 3 months; at least 16 natural teeth; moderate to severe chronic periodontitis, defined by loss of clinical attachment of $>5$ $\mathrm{mm}$ on 2 separate teeth, and no treatment within 6 months; able and willing to comply with study requirements (instructions and attendance); written informed consent; females to test negative for pregnancy, before and during the study period; use of effective birth control

Exclusion criteria: Evidence of chronic medical or psychiatric condition to prevent active study participation; TPOab positive; baseline serum level of TSH $<0.35$ or $>5.5$; antibiotics 3 months prior, or any other systemic condition requiring antibiotic premedication; history of thyroid disease; purported sensitivity or allergy to iodine; known sensitivity or allergy to shellfish; history of autoimmune disease, chronic infection (eg HIV or hepatitis), CVD (in prior 3 months), stroke or history/treatment for transient ischaemic attacks (in prior 3 months), or pulmonary embolus (in prior 6 months), angina pectoris, renal disease: serum creatinine exceeding $1.4 \mathrm{mg} / \mathrm{dl}$ (women) or $1.5 \mathrm{mg} / \mathrm{dl}$ (men), or currently receiving dialysis; periodontal disease, rampant caries, tissue damage created by poor oral care or treatment, soft or hard tissue tumours; mucosal tissue ulcerations, inflammation, or canker sores; orthodontic (including removable) appliances impinging on oral tissues; history of alcohol abuse or drug abuse; use of concomitant medication that, in the opinion of the investigator, might interfere with the outcome of the study (eg antibiotics, immuno-suppressants, steroids, or therapeutic doses of non-steroidal anti-inflammatory agents, phenytoin, calcium antagonists, cyclosporine or coumadin); concomitant therapy with another investigational drug or device without prior approval from the sponsor within 4 weeks prior to Visit 2 (Study Day 1); concomitant endodontic or periodontal therapy other than prophylaxis in the past 6 months; pregnant or nursing mothers, or intention to become pregnant during the study; residence in the same household as a subject already enrolled in the study (inclusion may create blinding and/or compliance issues)

Age at baseline: Unknown

Sex (M:F): Unknown

Tobacco use: Unknown

Alcohol consumption: Unknown, participants with history of alcohol abuse excluded though

Diabetes type: T2 DM

Duration since diabetes diagnosis: $>3$ months

Metabolic control: Poor mean $\mathrm{HbA1C}$ at baseline

Mean HbA1c at baseline: Gp A: 8.77 (SD 0.37); Gp B: 8.48 (SD 0.38)

Antidiabetic therapy: Not reported

Other clinical investigations: Suppuration; systemic biomarkers (LPS, TNF-a, CRP, IL-6, serum insulin and glucose); staining, calculus, opportunistic Candida infection; and patient satisfaction

Number randomised: 30

Number evaluated: 27

Interventions

Comparison: SRP + iodine rinse versus SRP + placebo rinse

Gp A $(n=15)$ : Standard periodontal therapy including SRP, Frio (locide) mouthrinse (molecular free iodine antibacterial rinse, $15 \mathrm{ml} \times 2$ daily for 90 days)

Gp B ( $n=15)$ : Standard periodontal therapy including SRP, placebo mouthrinse ( $15 \mathrm{ml} \times 2$ daily for 90 days)

Duration of follow-up: 6 months 
NCT00801164 (Continued)

Secondary: CAL, PPD, PI, GI (currently unpublished)
Trial ID: NCT00801164; HSC20080508H (study title: Exploratory study of iocide oral rinse in a diabetic population)

Patent information on Frio rinse product: www.freepatentsonline.com/WO2008005059.pdf

Adverse events: Not reported

Data analysis: Per-protocol

Conflict of interests: Unknown

SES: Unknown

Sample size calculation: Unknown. Trial register indicates 12 patients required per arm Contact information: Thomas W Oates (oates@uthscsa.edu)

Emailed. Response received. Full existing study report (student thesis) not available until published in a journal.

Quote: "We have not published this study other than as a student's masters thesis, I am sorry to say. However we have completed it. Here are the results for A1c - treatment was completed by 3 weeks from baseline:

HbA1c (\%): Baseline; 2 months; 3 months; 6 months

locide: 8.771 (SD 0.365); 8.529 (SD 0.380); 8.714 (SD 0.468); 9.136 (SD 0.468)

Placebo: 8.477 (SD 0.379); 8.415 (SD 0.394); 8.662 (SD 0.486); 8.800 (SD 0.485)

There were no significant differences between groups or time points

This was a randomized study using a computer generated list with examiners and operators blinded to allocations. 30 patients in total were entered into the study, with 27 completers (14 test, 13 control)"

\section{Risk of bias}

\begin{tabular}{|c|c|c|}
\hline Bias & Authors' judgement & Support for judgement \\
\hline $\begin{array}{l}\text { Random sequence genera- } \\
\text { tion (selection bias) }\end{array}$ & Low risk & $\begin{array}{l}\text { Correspondence: "..using a computer generated list with examiners and oper- } \\
\text { ators blinded to allocations" }\end{array}$ \\
\hline \multirow[t]{2}{*}{$\begin{array}{l}\text { Allocation concealment } \\
\text { (selection bias) }\end{array}$} & Unclear risk & $\begin{array}{l}\text { Correspondence: "...using a computer generated list with examiners and oper- } \\
\text { ators blinded to allocations" }\end{array}$ \\
\hline & & Comment: Unclear whether this refers to allocation concealment or blinding \\
\hline Blinding of participants & Low risk & $\begin{array}{l}\text { Unknown, although provided with a placebo rinse, so no reason to indicate } \\
\text { they were aware of their arm allocation }\end{array}$ \\
\hline \multirow[t]{2}{*}{$\begin{array}{l}\text { Blinding of clinical opera- } \\
\text { tor }\end{array}$} & Unclear risk & $\begin{array}{l}\text { Correspondence: "...using a computer generated list with examiners and oper- } \\
\text { ators blinded to allocations" }\end{array}$ \\
\hline & & $\begin{array}{l}\text { Comment: Insufficient information. Unclear whether this refers to allocation } \\
\text { concealment or blinding }\end{array}$ \\
\hline
\end{tabular}

\section{Incomplete outcome data High risk} (attrition bias)

All outcomes
Per-protocol analysis: not all participants analysed in groups randomised to, regardless of intervention actually received

27 of 30 completed the study, with no indication of reasons for drop-outs; despite this the rates are similar. Data presented by email for $\mathrm{Gp} \mathrm{An}=14, \mathrm{Gp} \mathrm{B} n$ $=13$

Selective reporting (re- Unclear risk Unknown until study published


NCT00801164 (Continued) Other bias Unclear risk $\quad \begin{aligned} & \text { Unpublished data, and therefore without peer-review although study lead } \\ & \text { confirms intention to publish }\end{aligned}$

Location: Brazil

Setting: Not reported

Number of centres: 1, University of Sao Paulo-Ribeirao Preto, Sao Paulo, Brazil

Recruitment period: Not stated

Funding source: Grant from Sao Paulo Research foundation (04/09844-8), National Council for Scientific and Technological Development (470638/2006) and a scholarship from Coordination for the Improvement of Graduated Personnel. Doxycycline was donated by Pfizer, Brazil

Participants

Inclusion criteria: Type $2 \mathrm{DM}$ diagnosed for $>5$ years and $\mathrm{HbA1C}>8 \%$, at least 1 site with probing depth $\geq 5 \mathrm{~mm}$, and 2 teeth with attachment loss $\geq 6 \mathrm{~mm}$

Exclusion criteria: The use of antibiotics or periodontal treatment in previous 6 months; smoking within past 5 years; pregnancy or lactation; major diabetic complications; concomitant medical therapy

Age at baseline: Overall: mean 52.9 yrs (SD ?); Gp A: mean 53.5 yrs (SD 13.6); Gp B: mean 52.3 yrs (SD 6.3)

Sex (M:F): Overall: M14:F16; Gp A M8:F7; Gp B M6:F9

Tobacco use: Non-smokers, required by exclusion criteria

Alcohol consumption: Not reported

Diabetes type: All T2 DM

Duration since diabetes diagnosis: $>5$ years

Metabolic control: Poor mean $\mathrm{HbA1c}$ at baseline

Mean HbA1c at baseline: Gp A 10.7\% (SD 2.0) 2.0; Gp B 11.8\% (SD 1.6)

Antidiabetic therapy: Not reported

Other medical conditions: Unlikely as concomitant medical therapy is exclusion criterion

Other clinical investigations: Suppuration, missing teeth, serum biomarkers

Number randomised: 35

Number evaluated: 30

Gp A ( $n=15$ ): SRP (in 2-4 sessions within 24 to 36 hours, using hand instruments and ultrasonic device, under local anaesthesia) $+\mathrm{OHI}$ and prophylaxis $(\mathrm{OHI}$ and scale and polish delivered every 2 weeks for 3 months) + doxycycline ( $200 \mathrm{mg}$ : 1 day prior to SRP, then $100 \mathrm{mg}$ daily for 14 days)

$\mathrm{Gp} \mathrm{B}(\mathrm{n}=15): \mathrm{SRP}+\mathrm{OHI}+$ prophylaxis with placebo as above

Duration of follow-up: 3 months 
O'Connell 2008 (Continued)

Outcomes

Primary: $\mathrm{HbA1c}$ at baseline and 3 months

Secondary: CAL, PPD, PI, BOP at baseline and 3 months

\section{Notes}

Sample size calculation: Not reported

Data analysis: Per-protocol

Adverse events: Not reported

SES: Not reported

HbA1c assessment method: High pressure liquid chromatography (Labtest Sistemas para Diagnostico, Lagoa Santa, MG, Brazil)

Conflict of interests: Authors declare no conflict of interests exist

\section{Risk of bias}

\begin{tabular}{|c|c|c|}
\hline Bias & Authors' judgement & Support for judgement \\
\hline $\begin{array}{l}\text { Random sequence genera- } \\
\text { tion (selection bias) }\end{array}$ & Unclear risk & Quote: "Subjects were randomly assigned to two groups" \\
\hline $\begin{array}{l}\text { Allocation concealment } \\
\text { (selection bias) }\end{array}$ & Unclear risk & Not reported \\
\hline Blinding of participants & Unclear risk & Quote: "double-masked, placebo-controlled study" \\
\hline $\begin{array}{l}\text { Blinding of clinical opera- } \\
\text { tor }\end{array}$ & Unclear risk & $\begin{array}{l}\text { Same operator performed SRP sessions. Not reported whether operator was } \\
\text { blinded as part of "double-masked" study }\end{array}$ \\
\hline \multirow{3}{*}{$\begin{array}{l}\text { Incomplete outcome data } \\
\text { (attrition bias) } \\
\text { All outcomes }\end{array}$} & High risk & $\begin{array}{l}\text { Per-protocol analysis: not all participants analysed in groups randomised to, } \\
\text { regardless of intervention actually received }\end{array}$ \\
\hline & & $\begin{array}{l}\text { Quote: "Two subjects were eliminated because they did not finish the treat- } \\
\text { ment phase, two subjects eliminated because they had to use an anticoagu- } \\
\text { lant agent, and one subject died" }\end{array}$ \\
\hline & & $\begin{array}{l}\text { Comment unclear which groups, and whether they could have obtained out- } \\
\text { come data for subjects who did not finish treatment phase }\end{array}$ \\
\hline $\begin{array}{l}\text { Selective reporting (re- } \\
\text { porting bias) }\end{array}$ & Unclear risk & All outcomes reported except adverse events \\
\hline Other bias & Low risk & No other apparent biases \\
\hline
\end{tabular}

Pradeep 2013

Methods

Trial design: 2-arm, single-centre, parallel-design RCT

Location: India

Setting: Hospital

Number of centres: 1 , outpatient section of Department of Periodontics, Government Dental College and Research Institute, Bangalore, India

Recruitment period: January 2011 to September 2011 
Funding source: Not reported. States Simvastatin (SMV) sample was provided by Aurobindo Pharma, Hyderabad, Andhra Pradesh, India (www.aurobindo.com/)

\section{Participants} Inclusion criteria: Patients with well-controlled type 2 diabetes; with $\mathrm{PD}>5 \mathrm{~mm}$ or $\mathrm{CAL}>4 \mathrm{~mm}$ and vertical bone loss $>3 \mathrm{~mm}$; no history of periodontal therapy or use of antibiotics in prior 6 months; and with $>20$ teeth

Exclusion criteria: Known systemic disease; known/suspected allergy to SMV; on systemic SMV/statin therapy; hyperlipidaemia or on a lipid-lowering diet; aggressive periodontitis; use of tobacco in any form; alcoholics; immunocompromised patients; pregnant or lactating; teeth with furcation defects, gingival recession, endodontic involvement or third molars

Age at baseline: Overall: range 30-50 years; data not provided by group

Sex (M:F): Overall: M20:F18; data not provided by group

Tobacco use: Exclusion criteria prevented participation of patients who use any form of tobacco

Weight: Not reported

BMI: Not reported

Alcohol consumption: Not reported

Diabetes type: All T2 DM

Duration since diabetes diagnosis: Not reported

Metabolic control: Good mean $\mathrm{HbA1C}$ at baseline

Mean HbA1c at baseline: Gp A: 6.66 (SD 0.11); Gp B: 6.71 (SD 0.13)

Quote: "Patients with well-controlled type 2 diabetes were selected"

Antidiabetic therapy: Not reported

Other investigations: Complete bone defect fill; intrabony defect (IBD) fill

Other medical conditions: Not reported

Number randomised: 38 (Gp A n = 19 (36 sites); Gp B n = 19 (34 sites))

Number evaluated: 35 (Gp A n = 17 (29 sites); Gp B $n=18$ (29 sites))

Lost to follow-up: 3 (Gp A $n=2 ; G p$ B: $n=1)$

Gp A ( $n=19)$ : OHI, followed by SRP and then adjunctive local delivery of 1.2\% SMV gel syringe-injected (by blunt cannula) into periodontal pockets

Gp B ( $n=19): O H I$, followed by SRP and then adjunctive local placement of placebo gel

Duration of follow-up: 9 months

Outcomes

Primary: Change in HbA1c from baseline to 3, 6, and 9 months

Secondary: Change in PI, GI (reported as modified sulcus bleeding index (mSBI)), PD and CAL from baseline to 3,6 , and 9 months

Notes

Sample size calculation: Number of participants required unknown as calculation was performed for sites rather than patients in each group ( $90 \%$ power to detect mean differences between groups). Planned sample size was 60 sites ( 30 in each arm). After loss to follow-up, trial presents data for 29 sites in each arm

SES: Not reported

Data analysis: Assumed ITT 
Pradeep 2013 (Continued)

Adverse events: Quote: "All participants tolerated the drug well without any complications or adverse reactions to the drug. Soft tissues healed within normal limits, and no significant visual differences were noted"

HbA1c assessment method: Not reported

Conflict of interests: Authors declare no conflict of interests

\section{Risk of bias}

\begin{tabular}{|c|c|c|}
\hline Bias & Authors' judgement & Support for judgement \\
\hline $\begin{array}{l}\text { Random sequence genera- } \\
\text { tion (selection bias) }\end{array}$ & Low risk & Randomisation conducted by computer-generated random numbers table \\
\hline $\begin{array}{l}\text { Allocation concealment } \\
\text { (selection bias) }\end{array}$ & Low risk & $\begin{array}{l}\text { Randomisation process conducted by external statistical unit, and states in- } \\
\text { vestigators were neither involved in the randomisation process, nor aware of } \\
\text { group assignment when assessing outcomes }\end{array}$ \\
\hline Blinding of participants & Low risk & $\begin{array}{l}\text { States participants masked to allocation, and only difference between inter- } \\
\text { vention and control group delivery was use of a placebo gel }\end{array}$ \\
\hline $\begin{array}{l}\text { Blinding of clinical opera- } \\
\text { tor }\end{array}$ & Unclear risk & Not reported \\
\hline $\begin{array}{l}\text { Incomplete outcome data } \\
\text { (attrition bias) }\end{array}$ & Unclear risk & Analysis assumed to have been ITT, but not specifically reported \\
\hline All outcomes & & $\begin{array}{l}3 \text { patients lost to follow-up, but clearly identified in patient flow diagram } \\
\text { which arms they were from (Intervention } n=2 ; \text { Control } n=1 \text { ) }\end{array}$ \\
\hline $\begin{array}{l}\text { Selective reporting (re- } \\
\text { porting bias) }\end{array}$ & Low risk & All specified outcomes (including adverse events) reported \\
\hline Other bias & Low risk & No other apparent biases \\
\hline
\end{tabular}

Raman 2014

Methods Trial design: 2-arm, multicentre, parallel-design RCT

Location: Malaysia

Setting: Hospital

Number of centres: 2, patients recruited from outpatient Diabetes Clinic of the University of Malaya Medical Centre, then treated at Periodontology Clinic at the Faculty of Dentistry, University of Malaya

Recruitment period: Recruitment period not explicit, although states screening and treatment from May 2010 - April 2011

Funding source: 2 research grants from University of Malaya (P0027/2009B and RG/11HTM)

Exclusion criteria: Systemic antibiotic use in prior 4 months; pregnancy; current smoker; cardiovascular/cerebrovascular event in prior 12 months; diabetes medication change during study; non-surgical periodontal therapy in prior 6 months; surgical periodontal therapy in prior 12 months

Age at baseline: Overall 56.2 yrs (SD 8.1); Gp A: 57.7 yrs (SD 9.9); Gp B: 54.6 yrs (SD 6.2) 
Tobacco use: Current smokers excluded from participation

Alcohol consumption: Not reported

Diabetes type: All type 2

Duration since diabetes diagnosis: Overall: $<7$ yrs $n=7(21.9 \%), 7-12$ yrs $n=8(25.0 \%),>12$ yrs $n=$ $17(53.1 \%) ;$ Gp A: $<7$ yrs $n=4(26.7 \%), 7-12$ yrs $n=4(26.7 \%),>12$ yrs $n=7(46.7 \%) ; G p$ B: $<7$ yrs $n=3$ $(17.6 \%), 7-12$ yrs $n=4(23.5 \%),>12$ yrs $n=10(58.8 \%)$

Metabolic control: Fair mean $\mathrm{HbA1c}$ at baseline

Mean HbA1c at baseline: Gp A: 7.80 (SD 1.50); Gp B: 7.60 (SD 1.50)

Antidiabetic therapy: Not reported fully. Only a quote: "All subjects who completed the study were on oral hypoglycaemic drugs"

Other medical conditions: Not reported

Other clinical investigations: Systemic hs-CRP, GBI

Number randomised: 40

Number evaluated: 32 (Gp A: $n=15 ; G p$ B: $n=17)$

Gp A ( $n=20)$ : Repeat OHI (modified Bass technique, soft-bristled toothbrush, compact-tuft toothbrush, interdental brush, floss (using TePe oral hygiene education set)) until PI $<20 \%$, followed by SRP (single visit, ultrasonic scaler, Gracey curettes) and $0.12 \%$ chlorhexidine mouthrinse (Hexipro, Evapharm, Kuala Lumpur, Malaysia) $3 \times 15 \mathrm{ml}$ p/d for 14 days. OHI repeated at each monthly visit

Gp B ( $n=20)$ : OHI (modified Bass technique, soft-bristled toothbrush, compact-tuft toothbrush, interdental brush, floss (using TePe oral hygiene education set)). OHI repeated at each monthly visit

Duration of follow-up: 3 months

Outcomes

Primary: HbA1c at baseline and 3 months

Secondary: PI, PPD, PAL (corresponds to CAL) at baseline, 2 months, and 3 months

Sample size calculation: 30 required (15 per arm; 80\% power). Accounting for attrition, recruited 40 (20 per arm). Results confirm arms were sufficiently powered after accounting for attrition. Quote: "This gave a within group analyses power of $80 \%$ for the NSPT group [Gp A] and $88 \%$ for the OHI group [Gp B]"

Data analysis: Per-protocol

SES: Ethnicity data provided. Overall: Malay $n=9(28.1 \%)$; Chinese $n=8(25 \%)$; Indian $n=6(46.9 \%)$ Gp A: Malay $n=5(33.3 \%)$; Chinese $n=4(26.7 \%) ;$ Indian $n=6(40.0 \%)$

Gp A: Malay n = 4 (23.5\%); Chinese $n=4(23.5 \%) ;$ Indian $n=9(52.9 \%)$

Adverse events: Not reported

HbA1c assessment method: Not reported. Assessed by private laboratory, using $15 \mathrm{ml}$ venous blood

Conflicts of interest: Authors declare no conflict of interests

Trial ID: NCT01951547

\section{Risk of bias}

Bias Authors' judgement Support for judgement

Treatment of periodontal disease for glycaemic control in people with diabetes mellitus (Review) 
Raman 2014 (Continued)

Random sequence genera- Low risk Quote: "All subjects were assigned via block randomisation to age matched tion (selection bias) NSPT and OHI groups. Following randomisation, baseline values for hs-CRP and $\mathrm{HbA} 1 \mathrm{c}$ were obtained"

\begin{tabular}{lll}
\hline $\begin{array}{l}\text { Allocation concealment } \\
\text { (selection bias) }\end{array}$ & Unclear risk & Not reported \\
\hline Blinding of participants & High risk & Not possible \\
\hline $\begin{array}{l}\text { Blinding of clinical opera- } \\
\text { tor }\end{array}$ & High risk & States "not double-blinded." Not reported further \\
\hline
\end{tabular}

Incomplete outcome data High risk

Per-protocol analysis: not all participants analysed in groups randomised to, All outcomes regardless of intervention actually received Gp A: lost 5 patients. 2 due to medication change during study (exclusion criteria); 2 withdrew for unspecified reasons; and 1 unable to attend recall due to distance

Gp B: lost 3 patients. 1 due to medication change during study; and 2 withdrew for unspecified reasons

\begin{tabular}{lll}
\hline $\begin{array}{l}\text { Selective reporting (re- } \\
\text { porting bias) }\end{array}$ & Unclear risk & All outcomes fully reported on, except adverse events \\
\hline Other bias & High risk & $\begin{array}{l}\text { Quote: "..during the randomization of subjects, more participants with poor } \\
\text { metabolic control were placed in the NSPT group. In the OHI group, there was } \\
\text { equal distribution of participants with poor and good metabolic control" }\end{array}$ \\
\hline
\end{tabular}

\section{Rocha 2001}

Methods Trial design: 2 -arm, single-centre, parallel-design RCT

Location: Mexico

Setting: Not reported

Number of centres: 1

Recruitment period: Not reported

Funding source: Grant from Consejo Nacional de Ciencia y Tecnologia (CONACYT) SIHGO 19980202026

Inclusion criteria: Patients aged 50-60 years with diagnosis of type 2 DM for a minimum of 5 years and established periodontitis, defined as probing depth $\geq 3 \mathrm{~mm}$ in at least 1 tooth. Patients did not have clinical evidence of complications of diabetes or systemic infections or other metabolic diseases. At least 6 teeth, gingival Index of 2-3 according to Silness and Loe, and gingival recession of 2-3 mm

Exclusion criteria: Smoking; recent peptic or oesophageal disorders; used drugs to inhibit gastric secretion for more than 2 weeks in previous 6 months; chronic treatment with NSAIDs estrogens or glucocorticoids

Age at baseline: Gp A mean 56 yrs (SD 3.5); Gp B mean 55 yrs (SD 3.6) $(P=0.22)$

Sex (M:F): M20:F20. Split by group not reported

Tobacco use: Non-smokers only

Alcohol consumption: Not reported 
Rocha 2001 (Continued)

\title{
Diabetes type: $\mathrm{T} 2 \mathrm{DM}$
}

Duration since diabetes diagnosis: $\geq 5$ years, mean 10.9 years $(5.8)$

Metabolic control: Poor mean $\mathrm{HbA1c}$ at baseline

Mean HbA1c at baseline: Gp A 11.9\% (SD 3.2); Gp B 13.1\% (SD 2.9) $(P=0.18)$

Antidiabetic therapy: Not reported

Other medical conditions: None

Other clinical investigations: Dental mobility, gingival recession, gingival bleeding

Number randomised: 40

Number evaluated: 40

\begin{abstract}
Interventions
Comparison: SRP + OHI + alendronate versus SRP + OHI + placebo

Gp A ( $n=20)$ : SRP (at baseline, by quadrant in 4 successive sessions) + OHI (instruction on mechanical toothbrushing and flossing) + alendronate $10 \mathrm{mg} /$ daily (aminobisphosphonate)

Gp B $(n=20)$ : SRP (at baseline, by quadrant in 4 successive sessions) + OHI (instruction on mechanical toothbrushing and flossing) + placebo capsules (1 capsule/daily; v trivitamin $100 \mathrm{mg}$ thiamine pyroxidine $50 \mathrm{mg}$ cyanocobalamin $250 \mu \mathrm{g}$ )
\end{abstract}

\section{Duration of follow-up: 6 months}

Subjects assessed for plaque fortnightly during 6-month study period, when flossing technique reinforced for each arm

\begin{tabular}{|c|c|c|}
\hline Outcomes & \multicolumn{2}{|c|}{$\begin{array}{l}\text { Primary: } \mathrm{HbA} 1 \mathrm{C} \text { (at baseline, and } 6 \text { months) } \\
\text { Secondary: } \mathrm{PD}, \mathrm{CAL} \text { (at baseline, and } 6 \text { months) }\end{array}$} \\
\hline Notes & $\begin{array}{l}\text { Sample size calculatic } \\
\text { Data analysis: Assume } \\
\text { SES: Not reported } \\
\text { HbA1c assessment me } \\
\text { souri, USA) } \\
\text { Conflict of interests: } \\
\text { medications under stu }\end{array}$ & $\begin{array}{l}\text { thod: Cation-exchange chromatography (Sigma Chemical Co, St. Louis, Mis- } \\
\text { ot reported, however does state "the companies producing or marketing the } \\
\text { ly were unaware of this work" }\end{array}$ \\
\hline \multicolumn{3}{|l|}{ Risk of bias } \\
\hline Bias & Authors' judgement & Support for judgement \\
\hline $\begin{array}{l}\text { Random sequence genera- } \\
\text { tion (selection bias) }\end{array}$ & High risk & $\begin{array}{l}\text { Quote: "..randomised double blind case control study" } \\
\text { Comment: Method of sequence generation not described and likely to be qua- } \\
\text { si random. Sex and age matched pairs before randomisation to trial arms }\end{array}$ \\
\hline $\begin{array}{l}\text { Allocation concealment } \\
\text { (selection bias) }\end{array}$ & High risk & Not reported \\
\hline Blinding of participants & Low risk & Quote: "Both patients and the examiner were blind to the treatment group" \\
\hline $\begin{array}{l}\text { Blinding of clinical opera- } \\
\text { tor }\end{array}$ & Unclear risk & Blinding possible, but not reported \\
\hline
\end{tabular}


Rocha 2001 (Continued)

$\begin{array}{ll}\begin{array}{l}\text { Incomplete outcome data } \\ \text { (attrition bias) }\end{array} & \text { Low risk } \\ \text { All outcomes } & \begin{array}{l}\text { Analysis assumed to have been ITT, but not specifically reported Withdrawals } \\ \text { and drop-outs not mentioned but numbers included in outcome evaluation } \\ \text { probably include all as degree of freedom for paired t-test is } 19\end{array}\end{array}$

\begin{tabular}{lll}
\hline $\begin{array}{l}\text { Selective reporting (re- } \\
\text { porting bias) }\end{array}$ & Unclear risk & $\begin{array}{l}\text { Planned outcomes not clearly specified but expected outcomes reported. No } \\
\text { adverse events data reported for alendronate }\end{array}$ \\
\hline Other bias & Low risk & No other apparent biases \\
\hline
\end{tabular}

Rodrigues 2003

Trial design: 2-arm, single-centre, parallel-design RCT
Location: Brazil
Setting: Hospital
Number of centres: 1, University Hospital Sao Paulo, Brazil
Recruitment period: Not reported
Funding source: Grants from CAPES - Coordenação de Aperfeiçoamento de Pessoal de Nivel Superior
(Brazilian Ministry of Education organisation to fund graduate education), and FAPESP (Fundação de
Amparo à Pesquisa do Estado de São Paulo (São Paulo Research Foundation) - grant 2000/14108-8)

Participants

Inclusion criteria: Type 2 diabetes and periodontal disease defined as probing depth of more than 5 $\mathrm{mm}$ at least 1 site; 2 teeth with more than $6 \mathrm{~mm} \mathrm{CAL}$

Exclusion criteria: Antibiotics in the last 6 months, smoking, pregnant, insulin controlled or diagnosis of DM less than 5 years

Age at baseline: Not reported

Sex (M:F): Not reported

Tobacco use: Specified exclusion

Alcohol consumption: Not reported

Diabetes type: $T 2$ DM

Duration since diabetes diagnosis: At least 5 years

Metabolic control: Poor mean $\mathrm{HbAlc}$ at baseline Mean HbA1c at baseline: Gp A 9.5\% (SD 2.4), Gp B 8.8\% (SD 1.8)

Antidiabetic therapy: Insulin users excluded from participation, no other detail reported except that "alterations in [...] diabetes control were recorded" - but again, data not reported

Other medical conditions: Test group had more reported diabetic complications

Cardiac complications (44\% versus 6\%) and hypertension (55\% versus $40 \%$ )

Other clinical investigations: FBG, RAL, percentage of surfaces exhibiting biofilm, and suppuration

Number randomised: 30

Number evaluated: Unclear, assumed 30. No information re: attrition 
Rodrigues 2003 (Continued)

Gp A ( $n=15)$ : SRP (2 sessions, within 24-36 hours; using standard curettes, an ultrasonic device and under local anaesthesia) $+\mathrm{OHI}(x 7$; prior to baseline, and twice $\mathrm{p} /$ month for 3 months after SRP) + prophylaxis ( 6 6; twice $p /$ month for 3 months after SRP) + systemic amoxicillin/clavulanic acid (given 24 hours prior to SRP, $875 \mathrm{mg}$ bid for 14 days)

Gp B ( $n=15)$ : SRP (2 sessions, within 24-36 hours; using standard curettes, an ultrasonic device and under local anaesthesia) + OHI ( $x$; p prior to baseline, and twice $p /$ month for 3 months after SRP) + prophylaxis ( 6 ; twice $p /$ month for 3 months after SRP)

Duration of study: 3 months

\begin{tabular}{ll} 
Outcomes & Primary: HbA1c (at baseline and 3 months) \\
& Secondary: PD, BOP (at baseline and 3 months) \\
\hline Notes & Sample size calculation: Not reported \\
& Data analysis: Assumed ITT \\
& HbA1c assessment method: High pressure liquid chromatography (Labtest Sistemas para Diagnostico, \\
& Lagoa Santa, MG, Brazil)
\end{tabular}

SES: Not reported

Conflict of interests: Not reported

\section{Risk of bias}

\begin{tabular}{|c|c|c|}
\hline Bias & Authors' judgement & Support for judgement \\
\hline $\begin{array}{l}\text { Random sequence genera- } \\
\text { tion (selection bias) }\end{array}$ & Low risk & $\begin{array}{l}\text { Confirmed in correspondence: "...the groups were randomized in advance of } \\
\text { the subject selection and then treated following the randomization table" }\end{array}$ \\
\hline $\begin{array}{l}\text { Allocation concealment } \\
\text { (selection bias) }\end{array}$ & Unclear risk & Not reported \\
\hline Blinding of participants & High risk & No placebo tablets \\
\hline $\begin{array}{l}\text { Blinding of clinical opera- } \\
\text { tor }\end{array}$ & Low risk & Confirmed in correspondence \\
\hline $\begin{array}{l}\text { Incomplete outcome data } \\
\text { (attrition bias) } \\
\text { All outcomes }\end{array}$ & Unclear risk & $\begin{array}{l}\text { Analysis assumed to have been ITT, but not specifically reported. Attrition not } \\
\text { reported. Unclear how many in each group at } 3 \text { months }\end{array}$ \\
\hline $\begin{array}{l}\text { Selective reporting (re- } \\
\text { porting bias) }\end{array}$ & Unclear risk & $\begin{array}{l}\text { No tabulated data, only extractable from core text of report. States alterations } \\
\text { in diabetes control/antibiotic use were recorded, but not presented. No re- } \\
\text { porting of adverse events }\end{array}$ \\
\hline Other bias & Unclear risk & Participant characteristics not reported \\
\hline
\end{tabular}

Santos 2009

Methods

Trial design: 2 -arm, single-centre, parallel-design RCT

Location: Brazil

Setting: Hospital 
Recruitment period: Not reported

Funding source: Not reported

Participants
Inclusion criteria: Participants aged 36-70 years with type 2 diabetes diagnosed within past 5 years and chronic periodontitis based on 1999 World Workshop Classification of Periodontal Disease; $>30$ years old with $\geq 15$ standing teeth (excluding third molars and teeth with severe periodontitis and/or caries with an indication for surgical extraction); $30 \%$ of sites required to have $\geq C A L$ of $5 \mathrm{~mm}$ at baseline

Exclusion criteria: Pregnancy; lactation; current smoking or smoking within the previous 5 years; periodontal or antibiotic therapy in the previous 6 months (including mouthrinses etc containing antimicrobials within the previous 2 months); any systemic condition affecting the progression of periodontal disease except DM; patients taking anti-inflammatory or immunosuppressive medications. Also excluded were people with periapical pathology, orthodontic treatment and those with multiple systemic diabetic complications

Age at baseline: Gp A mean 52.3 yrs (SD 9.4); Gp B mean 53.0 yrs (SD 9.2)

Sex (M:F): Overall: M16:F20 (44\%:56\%). Gp A M8:F10; Gp B M8:F10

Tobacco use: Specified exclusion

Alcohol consumption: Not reported

Diabetes type: T2 DM

Duration since diabetes diagnosis: $\leq 5$ years

Metabolic control: Poor mean $\mathrm{HbA1c}$ at baseline

Mean HbA1c at baseline: Gp A: 9.10 (SD 2.10); Gp B: 9.20 (SD 1.90)

Classified in study as:

Poor (HbA1c 9\%-12\%) Gp A 11, Gp B 10

Better (HbA1c 4.8\%-8.7\%) Gp A 7, Gp B 8

Antidiabetic therapy: All in receipt of oral hypoglycaemic medications, insulin, diet or combination

Other medical conditions: None

Other clinical investigations: FBG, suppuration on probing (SUP)

Number randomised: 36

Number evaluated: 36
Comparison: Immediate SRP (24 hrs) + OHI (x 3) + prophylaxis (x 2) versus ongoing SRP (21 days) $+\mathrm{OHI}(\times 3)+$ prophylaxis (x 2)

Gp A ( $n=18)$ : "Full-mouth" SRP (under local anaesthetic, completed in 2 sessions lasting 2 hours each, within 24 hours) $+\mathrm{OHI}$ ( $\times 3$ : at baseline, 3 and 6 months; brushing technique instruction for soft manual toothbrush, dental floss, interdental brushes, and provision of branded toothpaste for duration of study period) + supportive prophylaxis ( $\times 2$ : at 3 and 6 months, professional plaque control including abrasive sodium carbonate air-powder system)

Gp B ( $n=18)$ : "Partial" SRP (under local anaesthetic, completed in 4 sessions lasting 1 hour each, within a maximum of 21 days) $+\mathrm{OHI}$ ( $\times 3$ : at baseline, 3 and 6 months; brushing technique instruction for soft manual toothbrush, dental floss, interdental brushes, and provision of branded toothpaste for duration of study period) + supportive prophylaxis (x 2: at 3 and 6 months, professional plaque control including abrasive sodium carbonate air-powder system)

Duration of follow-up: 6 months 
Santos 2009 (Continued)

Outcomes

Primary outcome: HbA1c (at baseline, 3 and 6 months)

Secondary outcomes: PI, BOP, PD, CAL (at baseline, 3 and 6 months)

Notes
Sample size calculation: "The ideal sample size to ensure adequate power for this clinical trial was calculated considering differences $\geq 1 \mathrm{~mm}$ for CAL and a standard deviation of $0.94 \mathrm{~mm}$ between groups in initially deep periodontal pockets $(>6 \mathrm{~mm})$. Based on these calculations, it was decided that 14 subjects per group were necessary to provide $80 \%$ power with $a=0.05 "$

Data analysis: ITT

SES: Not reported

HbA1c assessment method: High pressure liquid chromatography

Adverse events: No adverse events occurred

Conflict of interests: Authors declare no conflict of interests exists

Note: Clarification sought from authors whether Santos 2009 and 2012 were the same study. Author confirmed they are indeed 2 separate studies on different participants (the difference being the lack of data collection for GCF and biofilm in Santos 2009), but that unfortunately the studies were not registered at the time to be able to reference trial IDs

\section{Risk of bias}

\begin{tabular}{|c|c|c|}
\hline Bias & Authors' judgement & Support for judgement \\
\hline $\begin{array}{l}\text { Random sequence genera- } \\
\text { tion (selection bias) }\end{array}$ & Low risk & Quote: "Toss of a coin" \\
\hline $\begin{array}{l}\text { Allocation concealment } \\
\text { (selection bias) }\end{array}$ & Unclear risk & Allocation concealed but no methods described \\
\hline Blinding of participants & High risk & Not possible \\
\hline $\begin{array}{l}\text { Blinding of clinical opera- } \\
\text { tor }\end{array}$ & High risk & Operative treatment differed between arms \\
\hline $\begin{array}{l}\text { Incomplete outcome data } \\
\text { (attrition bias) } \\
\text { All outcomes }\end{array}$ & Low risk & All participants completed study. ITT analysis \\
\hline $\begin{array}{l}\text { Selective reporting (re- } \\
\text { porting bias) }\end{array}$ & Low risk & Pre-specified outcomes reported, including adverse events \\
\hline Other bias & Low risk & No other apparent biases \\
\hline
\end{tabular}

Santos 2012

Methods Trial design: 2 -arm, single-centre, parallel-design RCT

Location: Brazil

Setting: Hospital

Number of centres: 1, Guarulhos University, Sao Paulo

Recruitment period: December 2007 until March 2009 

were receiving supplementation with insulin, an appropriate dietary regimen and/or oral hypoglycaemic agents. All subjects were $>30$ years of age, had at least 15 teeth (excluding third molars and teeth indicated to exodontias) and had more than $30 \%$ of sites with a probing depth and CAL of $>=4$ $\mathrm{mm}$ at baseline

Exclusion criteria: Pregnancy; lactation; current smoking and smoking within the past 5 years; periodontal and/or antibiotic therapy in the previous 6 months; regular use of mouthrinses containing antimicrobials in the preceding 2 months; other systemic condition that could affect the progression of periodontal disease; long-term treatment with anti-inflammatory and immunosuppressive medications; periapical pathology; orthodontic appliances and multiple systemic complications of DM

Age at baseline: Overall 42-67 years; Gp A mean 51.9 (SD) 7.8 years, Gp B mean 53.1 (SD) 8.1 years

Sex (M:F): Overall: M16:F18 (47\%:53\%). Gp A M9:F8; Gp B M7:F10

Tobacco use: Excluded

Alcohol consumption: Not reported

Diabetes type: T2 DM

Duration since diabetes diagnosis: >= 5 years

Metabolic control: Poor mean $\mathrm{HbA1c}$ at baseline

Mean HbA1c at baseline: Gp A: 9.40 (SD 2.50); Gp B: 8.90 (SD 2.20)

Antidiabetic therapy: All in receipt of oral hypoglycaemic medications, insulin, diet or combination

Other medical conditions: As stated in exclusion criteria

Other clinical investigations: SUP, FPG levels and measurements of cytokine- and osteoclastogenesis related factors (measured by gingival crevicular fluid)

Number randomised: 34

Number evaluated: 34

Interventions

Comparison: Immediate SRP (24 hrs) + OHI (x 4) + prophylaxis (x 3) versus ongoing SRP (21 days) $+\mathrm{OHI}$ (x 4) + prophylaxis (x 3)

Gp A ( $n=17)$ : "Full-mouth" SRP (under local anaesthetic, completed in 2 sessions lasting 2 hours each, within 24 hours) + OHI ( $x$ : at baseline, 3, 6 and 9 months; brushing technique instruction for soft manual toothbrush, dental floss, interdental brushes, and provision of branded toothpaste for duration of study period) + supportive prophylaxis ( $x$ 3: at 3, 6 and 9 months; professional plaque control including abrasive sodium carbonate air-powder system)

Gp B ( $n=17)$ : "Partial" SRP (under local anaesthetic, completed in 4 sessions lasting 1 hour each, within a maximum of 21 days) $+\mathrm{OHI}$ ( $x$ 4: at baseline, 3, 6 and 9 months; brushing technique instruction for soft manual toothbrush, dental floss, interdental brushes, and provision of branded toothpaste for duration of study period) + supportive prophylaxis ( $x$ 3: at 3, 6 and 9 months; professional plaque control including abrasive sodium carbonate air-powder system)

Duration of follow-up: 12 months

$\begin{array}{ll}\text { Outcomes } & \text { Primary: } \mathrm{HbA} 1 \mathrm{c} \text { (at baseline, 3, } 6 \text { and } 12 \text { months) } \\ & \text { Secondary: CAL, PI, BOP, PPD (at baseline, 3, } 6 \text { and } 12 \text { months) }\end{array}$

Notes

Sample size calculation: Quote: "..differences of at least $1 \mathrm{~mm}$ for clinical attachment level and a standard deviation of $1 \mathrm{~mm}$ between groups in initially deep pockets ( $>=7 \mathrm{~mm}$ ). Based on these calculations, it was decided that 17 subjects per group would provide $80 \%$ power at a significance level of $5 \% "$ 
Santos 2012 (Continued)

\section{Data analysis: ITT}

SES: Quote: "the subjects included in the current investigation were predominantly from families of a low socioeconomic status who often lack financial, social and educational support and have inadequate or limited access to medical care"

Adverse events: No adverse events occurred

HbAlc assessment method: High pressure liquid chromatography

Conflict of interests: Not reported

Note: Clarification sought from authors whether Santos 2009 and 2012 were the same study. Author confirmed they are indeed 2 separate studies on different participants (the difference being the lack of data collection for GCF and biofilm in Santos 2009), but that unfortunately the studies were not registered at the time to be able to reference trial IDs

\section{Risk of bias}

\begin{tabular}{|c|c|c|}
\hline Bias & Authors' judgement & Support for judgement \\
\hline $\begin{array}{l}\text { Random sequence genera- } \\
\text { tion (selection bias) }\end{array}$ & Low risk & $\begin{array}{l}\text { Quote: "The subjects were randomly assigned, by tossing a coin (performed by } \\
\text { the same assessor (P.M.D.))" }\end{array}$ \\
\hline $\begin{array}{l}\text { Allocation concealment } \\
\text { (selection bias) }\end{array}$ & Unclear risk & No detail provided \\
\hline Blinding of participants & High risk & $\begin{array}{l}\text { Number of treatment appointments differed between arms so not possible to } \\
\text { blind participants }\end{array}$ \\
\hline $\begin{array}{l}\text { Blinding of clinical opera- } \\
\text { tor }\end{array}$ & High risk & Operative treatment differed between arms \\
\hline $\begin{array}{l}\text { Incomplete outcome data } \\
\text { (attrition bias) }\end{array}$ & Low risk & ITT analysis \\
\hline All outcomes & & $\begin{array}{l}\text { Quote: "There were no subject or site drop-outs during the study period. No } \\
\text { adverse effects, such as fever and indisposition after treatment, were report- } \\
\text { ed by any subject, and no changes in the category of treatment regimen for DM } \\
\text { occurred during the study" }\end{array}$ \\
\hline $\begin{array}{l}\text { Selective reporting (re- } \\
\text { porting bias) }\end{array}$ & Low risk & Pre-specified outcomes reported, including adverse events \\
\hline Other bias & Low risk & No other apparent biases \\
\hline
\end{tabular}

Location: Brazil

Setting: Hospital

Number of centres: 1 , Clinic of Guarulhos University

Recruitment period: July 2008 - May 2010

Funding source: Sao Paulo Research Foundation (\#2008/09687-0 2008/11419-4) 
Santos 2013 (Continued)

Participants
Inclusion criteria: $\geq 35$ years old; $\geq 15$ teeth present after excluding third molars and teeth requiring exodontia due to advanced caries; diagnosis of generalised chronic periodontitis (Armitage 1999); concomitant PPD and CAL $\geq 4 \mathrm{~mm}$ in at least $30 \%$ of sites; presenting with T2 DM in the last 5 years; must be on insulin, diet controlled or oral hypoglycaemic agents

Exclusion criteria: Pregnancy, lactation, smokers or those that had smoked in last 5 years. SRP in last 12 months. Antimicrobial therapy in previous 6 months including those with medical conditions requiring antibiotic cover and use of antimicrobial mouthwashes in last 3 months. Medical conditions that affect the progress of periodontitis and long-term administration of anti-inflammatory and immunosuppressive medications. Allergy to CHX. Periapical pathology or wearing orthodontic appliances. Major complications of DM

Age at baseline: Gp A mean 50.3 yrs (SD 9.5); Gp B 53.9 yrs (SD 10.8)

Sex (M:F) Overall: M10:F28; Gp A M4:F15; Gp B M6:F13

Tobacco use: Excluded from study

Alcohol consumption: Not recorded

Diabetes type: Type 2 DM

Duration since diabetes diagnosis: Gp A mean 6.3 yrs (SD 0.8); Gp B mean 6.8 yrs (SD 1.1)

Metabolic control: Poor mean $\mathrm{HbA1c}$ at baseline

Mean HbA1c at baseline: Gp A 10.00\% (SD 2.41); Gp B 10.4\% (SD 2.9)

HbAlc >8.0\%: Gp A 15/19, Gp B 13/19

Antidiabetic therapy: All in receipt of oral hypoglycaemic medication, insulin, diet or combination

Other clinical investigations: FPG (mg/dl), SUP (\%)

Number randomised: 38

Number evaluated: 3 months $=37,6$ months $=37,12$ months $=33$

(Gp A: 2 lost to follow-up; Gp B: 3 lost to follow-up (1 excluded from analysis due to not attending 3month assessment))

ITT analysis used for all results using 6-month data

Interventions

Comparison: SRP + FMD (CHX rinse x 60 days \& CHX gel x 1) + OHI (x 4) + prophylaxis (x 3) versus SRP + placebo FMD (rinse $x 60$ days \& gel $x 1)+$ OHI $(x 4)+$ prophylaxis $(x 3)$

Gp A ( $n=19):$ SRP + FMD (full mouth disinfection with chlorhexidine digluconate (CHX) $0.12 \%$ rinse for 60 days, and $\mathrm{CHX}$ gel ( $1 \%$, applied to irrigate all treated pockets 3 times, within 10 minutes)) $+\mathrm{OHI}(\mathrm{X} 4$ : at baseline, 3, 6 and 9 months; brushing technique instruction for soft manual toothbrush, dental floss, interdental brushes, and provision of branded toothpaste for duration of study period) + supportive prophylaxis ( $\times 3$ : at 3, 6 and 9 months; professional plaque control including abrasive sodium carbonate air-powder system and subgingival debridement)

Gp B ( $n=19)$ : SRP + placebo FMD (placebo rinse for 60 days, and placebo gel (1\%, applied to irrigate all treated pockets 3 times, within 10 minutes: composed of aspartame, zinc acetate dehydrate, alcohol, colourant, flavouring, acesulphame and purified water)) $+\mathrm{OHI}$ ( $\mathrm{x}$ 4: at baseline, 3, 6 and 9 months; brushing technique instruction for soft manual toothbrush, dental floss, interdental brushes, and provision of branded toothpaste for duration of study period) + supportive prophylaxis (x 3: at 3, 6 and 9 months; professional plaque control including abrasive sodium carbonate air-powder system and subgingival debridement)

Duration of follow-up: 12 months 
SES: Not reported

Adverse events: Gp A: 89\%; Gp B: 67\% report taste perception change/dry mouth/staining

HbAlc assessment method: High pressure liquid chromatography

Conflict of interests: Authors declare no conflict of interests exists

\section{Risk of bias}

\begin{tabular}{lll}
\hline Bias & Authors' judgement & Support for judgement \\
\hline $\begin{array}{l}\text { Random sequence genera- } \\
\text { tion (selection bias) }\end{array}$ & Low risk & $\begin{array}{l}\text { Code number given a number during enrolment visit. Assignment by computer } \\
\text { generated table (PMD) }\end{array}$ \\
\hline $\begin{array}{l}\text { Allocation concealment } \\
\text { (selection bias) }\end{array}$ & Low risk & PMD allocated code bottles \\
\hline $\begin{array}{l}\text { Blinding of participants } \\
\text { Blinding of clinical opera- } \\
\text { tor }\end{array}$ & Low risk risk & Placebo given to control group \\
\hline $\begin{array}{l}\text { Incomplete outcome data } \\
\text { (attrition bias) } \\
\text { All outcomes }\end{array}$ & Low risk & $\begin{array}{l}\text { Both groups received similar mechanical therapy, and the operator did not } \\
\text { identify the contents of the bottles at any time during the study }\end{array}$ \\
\hline $\begin{array}{l}\text { Selective reporting (re- } \\
\text { porting bias) }\end{array}$ & Low risk & $\begin{array}{l}\text { ITT analysis. All patients accounted for. 5 patients lost to follow-up. Similar } \\
\text { rates, no concerns }\end{array}$ \\
\hline \begin{tabular}{l} 
Other bias \\
\hline
\end{tabular} & Low risk & All outcomes reported, including adverse events \\
\hline
\end{tabular}

Singh 2008

Methods Trial design: 3 -arm, single-centre, parallel-design RCT

Location: India

Setting: Hospital

Number of centres: 1, Department of Periodontics, JSS Dental College, Mysore, India

Recruitment period: Not reported

Funding source: Quote: "Source of support: Nil" examined teeth with $\geq 4 \mathrm{~mm}$ probing depth); absence of any major diabetic complications; no evidence of any systemic disease (other than diabetes) being a risk factor for periodontitis

Exclusion criteria: Uncontrolled DM; periodontal treatment in prior 6 months; antibiotic administration in prior 3 months; $<16$ remaining natural teeth 
Age at baseline: Not reported

Sex (M:F): Not reported

Tobacco use: Not reported

Alcohol consumption: Not reported

Diabetes type: Type 2 DM

Duration since diabetes diagnosis: Not reported

Metabolic control: Fair mean HbA1c at baseline

Mean HbAlc at baseline: Gp A: mean 7.9\% (SD 0.7); Gp B mean 8.3\% (SD 0.7); Gp C mean 8.08\% (SD 0.7)

Antidiabetic therapy: Not specifically reported. All in receipt of antidiabetic therapy but no indication what form ("Exclusion criteria: Patients with uncontrolled DM")

Other clinical investigations: FPG, PPBG

Number randomised: 45

Number evaluated: 45

\section{Comparison: SRP + OHI versus SRP + OHI + doxycycline versus no treatment}

Gp A ( $n=15)$ : Full mouth SRP (under local anaesthesia) + OHI

Gp B ( $n=15)$ : Full mouth SRP + OHI + systemic doxycycline (200 mg on treatment day, followed by 100 $\mathrm{mg} \mathrm{p} / \mathrm{d} \times 14$ days)

Gp C ( $\mathrm{n}=15)$ : No treatment

Note: Additionally "after oral examination the teeth with poor prognosis were extracted." No indication which Gps or how many patients received extractions, or whether this may have affected treatment outcomes

Duration of follow-up: 3 months

\begin{tabular}{|c|c|c|}
\hline Outcomes & \multicolumn{2}{|c|}{$\begin{array}{l}\text { Primary: } \mathrm{HbA} 1 \mathrm{c} \text { (at baseline and } 3 \text { months) } \\
\text { Secondary: PI, GI, PPD, CAL (at baseline and } 3 \text { months) }\end{array}$} \\
\hline Notes & $\begin{array}{l}\text { Sample size calculati } \\
\text { Data analysis: Assum } \\
\text { SES: Not reported } \\
\text { Adverse events: Quot } \\
\text { doxycycline" } \\
\text { HbA1c assessment m } \\
\text { Conflict of interests: }\end{array}$ & $\begin{array}{l}\text { n: Not reported } \\
\text { ITT } \\
\text { thod: Liquid chromatography } \\
\text { uthors declare no conflict of interests exists }\end{array}$ \\
\hline \multicolumn{3}{|l|}{ Risk of bias } \\
\hline Bias & Authors' judgement & Support for judgement \\
\hline $\begin{array}{l}\text { Random sequence genera- } \\
\text { tion (selection bias) }\end{array}$ & Unclear risk & $\begin{array}{l}\text { Quote: "They were randomly divided into three groups of } 15 \text { patients each" } \\
\text { Comment: No further details }\end{array}$ \\
\hline
\end{tabular}


Singh 2008 (Continued)

\begin{tabular}{lll}
$\begin{array}{l}\text { Allocation concealment } \\
\text { (selection bias) }\end{array}$ & Unclear risk & Not reported \\
\hline Blinding of participants & High risk & Not possible, and no placebos used \\
\hline $\begin{array}{l}\text { Blinding of clinical opera- } \\
\text { tor }\end{array}$ & High risk & Not reported, and unlikely to be possible due to differing treatments provided \\
\hline $\begin{array}{l}\text { Incomplete outcome data } \\
\text { (attrition bias) } \\
\text { All outcomes }\end{array}$ & Unclear risk & $\begin{array}{l}\text { No patients reported as lost to follow-up. Analysis assumed to have been ITT, } \\
\text { but not specifically reported }\end{array}$ \\
\hline $\begin{array}{l}\text { Selective reporting (re- } \\
\text { porting bias) }\end{array}$ & Unclear risk & $\begin{array}{l}\text { Planned outcomes reported for 3 months; however, assessed at } 1 \text { month and } \\
\text { not reported. Furthermore, no adverse events reported other than for doxycy- } \\
\text { cline use (Gp B) relating to SRP (Gps A+B) or no treatment (Gp C) }\end{array}$ \\
\hline $\begin{array}{l}\text { Other bias } \\
\text { Unclear risk }\end{array}$ & $\begin{array}{l}\text { No patient characteristics presented therefore unknown if baseline imbal- } \\
\text { ances between groups. Also no indication of how many patients in each arm } \\
\text { received tooth extractions as part of treatment protocol as wound healing may } \\
\text { potentially affect results }\end{array}$ \\
\hline
\end{tabular}

Skaleric 2004

Methods

Trial design: 2 -arm, single-centre, parallel-design RCT

Location: Slovenia

Setting: Hospital

Number of centres: 1 , Diabetes Clinic University of Ljubljana

Recruitment period: Not reported

Funding source: Not reported

Participants

Inclusion criteria: $>21$ years old; good general health; clinical diagnosis of periodontitis defined as 4 teeth in more than 2 quadrants with $\geq 5 \mathrm{~mm}$ periodontal pocketing, 2 of which had 6-9 $\mathrm{mm}$ pockets and BOP; uncontrolled or poorly controlled T1 DM as determined by HbA1c of $9 \%$; stable dosage of angiotensin II (ACE/AII) inhibitors or any other hypotensive; stable oral antidiabetic medications in last 2 months; no major control of dental infection (extraction, root canal therapy, local or systemic antibiotics).

Exclusion criteria: T2 DM. Pregnant, lactating or of child bearing potential and not using acceptable methods of birth control (hormonal, barrier or abstinence) and patients treated with medication known to affect periodontal status (eg phenytoin, calcium antagonists, Coumadin, cyclosporine, and NSAIDs) within 1 month of baseline visit. Presence of diabetes-related wounds and/or ulcers, requirement of prophylactic antibiotics, allergies to tetracyclines, steroid medications except for acute topical treatment. Use of systemic antibiotics within 3 months prior to enrolment, serum creatinine $\geq 1.9 \mathrm{mg} /$ $\mathrm{ml}$, laboratory values in excess of twice normal limit, and average baseline BP $>160 / 100$ limit. History of severe CVD within last 3 months, severe pulmonary disease, severe liver disease, ESRD (end stage renal disease), active malignancy, cerebral vascular disease, HIV, tuberculosis, hepatitis or other active infectious disease, drug or alcohol abuse, mental or cognitive deficiencies, or any condition which might require surgery during the course of the study and no major adverse events were reported

Age at baseline: Overall mean: $41.8 \mathrm{yrs}$ (SD 7.5); Gp A mean $42.0 \mathrm{yrs}$ (SD not reported); Gp B 41.6 yrs (SD not reported $)(P=0.98)$

Sex (M:F): Overall: M10:F10; Gp A M5:F5; Gp B M5:F5 (P = 1) 
Tobacco use: $10 / 20 \mathrm{Gp} \mathrm{A} 7, \mathrm{Gp} \mathrm{B} 3(\mathrm{P}=0.18)$

Alcohol consumption: Not reported

Diabetes type: Type $1 \mathrm{DM}$

Duration since diabetes diagnosis: $<5$ years: $n=4 ;>5$ years: $n=16$

Metabolic control: Poor mean HbA1c at baseline

Mean HbA1c at baseline: Gp A 9.11\% (SD 0.93); Gp B 9.49\% (SD 1.29)

Antidiabetic therapy: Not specifically reported, except for quotes: "Patients with type 2 non-insulin dependent diabetes were excluded from the study." "If patients were on additional oral antidiabetic medication, they had to be stable with no changes in the past two months."

Other clinical investigations: Sites with PD $>5 \mathrm{~mm}$

Number randomised: 20

Number evaluated: 20 at 3 months, 20 at 6 months phylaxis

Gp A ( $n=10)$ : SRP (within 48 hrs of baseline; using hand curettes, ultrasonic instruments and local anaesthesia) + minocycline hydrochloride microspheres (1 mg; controlled release bioresorbable polymer, at baseline and 12 weeks; in pockets $\geq 5 \mathrm{~mm}$ after completion of SRP and bleeding subsided participants instructed to avoid toothbrushing for $12 \mathrm{hrs}$, and flossing for 10 days post-treatment) + supragingival prophylaxis (at 12 weeks; full-mouth cleaning, for $20 \mathrm{~min}$ )

Gp B $(n=10)$ : SRP (within 48 hrs of baseline; using hand curettes, ultrasonic instruments and local anaesthesia) + supragingival prophylaxis (at 12 weeks; full-mouth cleaning, for $20 \mathrm{~min}$ )

Duration of follow-up: 6 months

Outcomes

Primary: $\mathrm{HbAlc}$ at baseline, 6 weeks, 12 weeks ( 3 months), 18 weeks, and 24 weeks ( 6 months)

Secondary: CAL, PD, PI, GI, at baseline, 12 weeks ( 3 months) and 24 weeks ( 6 months)

Data analysis: ITT

SES: Not reported

HbAlc assessment method: Not reported

Conflict of interests: Not reported

Adverse events: No major adverse events reported

Note: Use of antibacterial rinses, toothpastes (triclosan or $0.454 \%$ stannous fluoride) and irrigating devices were not allowed by patients during the study period

\section{Risk of bias}

\begin{tabular}{lll}
\hline Bias & Authors' judgement & Support for judgement \\
\hline $\begin{array}{l}\text { Random sequence genera- } \\
\text { tion (selection bias) }\end{array}$ & Unclear risk & Randomised by envelope. Inadequate description \\
\hline $\begin{array}{l}\text { Allocation concealment } \\
\text { (selection bias) }\end{array}$ & Unclear risk & Not reported \\
\hline
\end{tabular}


Skaleric 2004 (Continued)

\begin{tabular}{|c|c|c|}
\hline Blinding of participants & High risk & Not possible \\
\hline $\begin{array}{l}\text { Blinding of clinical opera- } \\
\text { tor }\end{array}$ & Unclear risk & Described as single blinded but no description \\
\hline $\begin{array}{l}\text { Incomplete outcome data } \\
\text { (attrition bias) } \\
\text { All outcomes }\end{array}$ & Low risk & All patients completed. ITT analysis \\
\hline $\begin{array}{l}\text { Selective reporting (re- } \\
\text { porting bias) }\end{array}$ & Low risk & $\begin{array}{l}\text { All pre-stated outcomes reported although pockets }>5 \mathrm{~mm} \text { were only de- } \\
\text { scribed in baseline data. Adverse events also reported }\end{array}$ \\
\hline Other bias & Low risk & No other apparent biases \\
\hline
\end{tabular}

\section{Sun 2011}

Methods

Trial design: 2-arm, single-centre, parallel-design RCT

Location: China

Setting: Hospital

Number of centres: 1, Second Affiliated Hospital, College of Medicine, Zhejiang University, China

Recruitment period: August 2008 to November 2010

Funding source: Grants from public research organisations: Zhejiang Science and Technology Projects (2009C33168), Natural Science Foundation of Zhejiang Province (Y2100077), Zhejiang Education Committee Projects (Y201017607), National Natural Science Foundation of China (30872884) and Zhejiang Health Bureau Fund (2009A104)

Participants

Inclusion criteria: Patients diagnosed with T2 DM at least 1 year prior to study; moderately poor glycaemic control (HbA1c between $7.5 \%$ and $9.5 \%$ ); aged 70 years; BMI $19-26 \mathrm{~kg} / \mathrm{m}^{2}$ in women, BMI $20-27$ $\mathrm{kg} / \mathrm{m}^{2}$ in men; no medication changes during the 3 months of study; not smoking; without severe complications, such as diabetic nephropathy, stroke, angina, myocardial infarction and so on. The diagnosis

of periodontitis met the following conditions: at least 20 teeth, $\mathrm{PD}>5 \mathrm{~mm},>30 \%$ teeth with attachment loss (AL) over $4 \mathrm{~mm}$, or $>60 \%$ teeth with $\mathrm{PD}>4 \mathrm{~mm}$ and $\mathrm{AL}>3 \mathrm{~mm}$; no periodontal treatment in the previous 6 months; no antibiotics or non-steroidal anti-inflammatory drugs administered in previous 3 months; no serious systemic diseases or complications

Exclusion criteria: Patients with systemic inflammatory diseases (rheumatoid arthritis, etc.), blood disease, liver damage, kidney disease or trauma

Age at baseline: Gp A mean 55.13 yrs (SD 11.16); Gp B mean 54.23 yrs (SD 10.85)

Sex (M:F): Overall: M67:F90; Gp A: M35:F47; Gp B: M32:F43

Tobacco use: Smokers excluded

Alcohol consumption: Not reported

Diabetes type: All T2 DM

Duration since diabetes diagnosis: >1 year

Metabolic control: Poor mean $\mathrm{HbA1c}$ at baseline

Mean HbA1c at baseline: Gp A: 8.75\% (SD 0.67); Gp B: 8.70\% (SD 0.65) 
Sun 2011 (Continued)

Antidiabetic therapy: Not reported, only study requirement for no medication changes during study period

\section{Other medical conditions: None}

Other clinical investigations: Sulcus bleeding index; fasting plasma glucose; triglycerides; total cholesterol; high-density lipoprotein cholesterol; low-density lipoprotein cholesterol; FINS, fasting insulin; homeostasis model assessment of insulin resistance; high-sensitivity $\mathrm{C}$ reactive protein; tumour necrosis factor; interleukin-6; adiponectin

Number randomised: 190

Number evaluated: 157

Interventions

Comparison: SRP + OHI + antibiotics versus no intervention

$\mathrm{Gp} \mathrm{A}$ ( $\mathrm{n}=82$ after removal of patients not completing the study): $\mathrm{OHI}$, full mouth scaling (supragingival and subgingival scaling), root planing, periodontal flap surgery when indicated, and extraction of hopeless teeth, restore of balanced occlusion. Antibiotics (tinidazole $1.0 \mathrm{~g}$, bid, po and ampicillin $0.25 \mathrm{~g}$, qid, po) were prescribed for 3 days before and after periodontal intervention. All periodontal interventions were performed by 1 periodontist

Gp B ( $n=75$ after removal of patients not completing the study): No periodontal treatment (no indication if $\mathrm{OHI}$ delivered)

Duration of follow-up: 3 months

Outcomes

Primary: $\mathrm{HbA} 1 \mathrm{C}$ at baseline and 3 months

Secondary: PD, CAL, BI, PI at baseline and 3 months

Notes

Sample size calculation: Not reported

Data analysis: Per-protocol

SES: Not reported

Adverse events: Not reported

HbA1c assessment method: Immunoturbidimetry

Conflict of interests: Authors declare no conflict of interests exists

Note: Not detailed anywhere how many were originally in each group

Quote: "A total of 33 patients did not finish the study. The reasons for dropping out included withdrawal due to personal reasons (such as sickness, no available time) (12 patients), later follow-up visit (21 patients, over 3 months). The data of these patients have been excluded from the data at the baseline (Table 1, 2)"

\section{Risk of bias}

\begin{tabular}{lll}
\hline Bias & Authors' judgement & Support for judgement \\
\hline $\begin{array}{l}\text { Random sequence genera- } \\
\text { tion (selection bias) }\end{array}$ & Unclear risk & $\begin{array}{l}\text { Quote; "..patients were randomly divided into two Groups." This is the only in- } \\
\text { formation reported. The study is not even described as being an RCT }\end{array}$ \\
\hline $\begin{array}{ll}\text { Allocation concealment } \\
\text { (selection bias) }\end{array}$ & Unclear risk & Not reported \\
\hline Blinding of participants & High risk & Quote: "study was not blinded" \\
\hline
\end{tabular}


Sun 2011 (Continued)
Blinding of clinical opera-
High risk
Quote: "study was not blinded"
tor

Incomplete outcome data High risk

(attrition bias)

All outcomes
Per-protocol analysis: not all participants analysed in groups randomised to, regardless of intervention actually received

All losses accounted for by rationale, but not indicated which arm withdrawals are from: personal reasons $n=12$; postponed follow-up visit $n=21$, over 3 months

\begin{tabular}{lll}
\hline $\begin{array}{l}\text { Selective reporting (re- } \\
\text { porting bias) }\end{array}$ & Unclear risk & All outcomes reported, except adverse events \\
\hline Other bias & Low risk & No other apparent biases
\end{tabular}

Tsalikis 2014

Methods Trial design: 2-arm, multicentre, parallel-design RCT

Location: Greece

Setting: Hospital

Number of centres: 2 , undergraduate and postgraduate clinics of the Department of Preventive Dentistry, Periodontology and Implant Biology, Dental School, Aristotle University of Thessaloniki, Greece; Pathology Clinic, Hippokrateion Hospital, Aristotle University of Thessaloniki, Greece

Recruitment period: Not reported

Funding source: Procter and Gamble Hellas (Koulourides 2011 Award for Dental Research, Greece)

\section{Participants}

Inclusion criteria: > 30 years; T2 DM (diagnosis at least 1 year prior to baseline); $\mathrm{HbA} 1 \mathrm{c}<7.5 \%$ from at least 2 consecutive patient medical record values; absence of important diabetes-related comorbidities; moderate or advanced periodontitis; absence of other systemic diseases known to be risk factors for periodontitis; written consensus; ability to attend recall visits

Exclusion criteria: T1 DM; antibiotics in prior 3 months; periodontal treatment in prior 6 months; smoking; infectious conditions (eg hepatitis, HIV); pregnant/lactating

Age at baseline: Overall: 60.4 yrs (SD 9.1); Gp A: 62.9 yrs (SD 10.0); Gp B: 57.9 yrs (SD 8.2)

Sex (M/F): Overall: M38:F28; Gp A: M18:F13; Gp B: M20:F15

Tobacco use: Smokers excluded from participation in study

Alcohol consumption: Not reported

Diabetes type: All T2 DM

Duration since diabetes diagnosis: Overall: 11.0 yrs (SD 5.8); Gp A: 11.8 yrs (SD 5.9); Gp B: 10.2 yrs (SD 5.7)

Metabolic control: Good mean $\mathrm{HbA1c}$ at baseline

Mean HbA1c at baseline: $6.70 \%$ (SD 0.61); Gp B: 6.89\% (SD 0.60)

Antidiabetic therapy: Not reported

Other medical conditions: Not specifically reported, although inclusion criteria specifies requirement for "Absence of important comorbidities due to diabetes"

Other investigations: Gingival recession 
Tsalikis 2014 (Continued)

\section{Number randomised: 70}

Number evaluated: 66 (Gp A: 31; Gp B: 35)

\section{Interventions \\ Comparison: SRP + OHI + adjunctive antimicrobial (doxycycline) versus SRP + OHI + placebo an- timicrobial}

Gp A ( $n=35)$ : 2-week OHI pretreatment phase (resulting in $<20 \%$ plaque for inclusion in trial), followed by SRP ( 2 sessions of supragingival Piezon ultrasonic instrumentation and subgingival hand instrumentation with Gracey curettes, under local anaesthetic), before randomisation to receive systemic doxycycline ( 21 days: $200 \mathrm{mg}$ loading dose, followed by $100 \mathrm{mg}$ for further 20 days) and subsequent OHI reinforcement after clinical assessments (patients provided with identical soft nylon multitufted Oral-B Indicator manual toothbrushes)

Gp B ( $n=35)$ : 2-week OHI pretreatment phase (resulting in <20\% plaque for inclusion in trial), followed by SRP ( 2 sessions of supragingival Piezon ultrasonic instrumentation and subgingival hand instrumentation with Gracey curettes, under local anaesthetic), before randomisation to receive systemic placebo ( 21 days: initial dose, followed by additional daily dose for further 20 days) and subsequent $\mathrm{OHI}$ reinforcement after clinical assessments (patients provided with identical soft nylon multitufted Oral-B Indicator manual toothbrushes)

\section{Outcomes}

Primary: $\mathrm{HbAlC}$ at baseline, 3 and 6 months

Secondary: PPD, CAL, BOP at baseline, 3 and 6 months

Duration of follow-up: 6 months

\section{Notes}

Sample size calculation: 60 required ( 30 per arm; 0.05 significance (2-tailed test) with $99 \%$ power to detect CAL means difference of $1.13 \mathrm{~mm}$ SD $1.0 \mathrm{~mm}$ ). Accounting for attrition, recruited 70 (35 per arm)

Data analysis: Per-protocol

Adverse events: Quote: "no major adverse effects were reported for both groups. Dizziness and difficulty to swallow was reported by one female participant in the control group (Gp B)"

SES: Not reported

HbA1c assessment method: A1CNow+ Multitest HbA1c system (Bayer HealthCare, Basel, Switzerland) in accordance with manufacturer instructions

Conflicts of interest: Authors declare no conflict of interests

Trial ID: Not reported

\section{Risk of bias}

\begin{tabular}{|c|c|c|}
\hline Bias & Authors' judgement & Support for judgement \\
\hline $\begin{array}{l}\text { Random sequence genera- } \\
\text { tion (selection bias) }\end{array}$ & Low risk & $\begin{array}{l}\text { Quote: "Randomization was generated using randomization software, } \\
\text { (www.randomization.com) and the randomization list was kept by one of the } \\
\text { authors (LT) until patients were eligible for the study" }\end{array}$ \\
\hline \multirow[t]{2}{*}{$\begin{array}{l}\text { Allocation concealment } \\
\text { (selection bias) }\end{array}$} & High risk & $\begin{array}{l}\text { Quote: "Upon completion of [mechanical] treatment, subjects were allocated } \\
\text { [to receive antibiotic or placebo] in the two groups by one of the authors (DS) } \\
\text { according to the randomization list kept by LT" }\end{array}$ \\
\hline & & Comment: Inadequate concealment due to list held by author \\
\hline Blinding of participants & Low risk & $\begin{array}{l}\text { Quote: "Subjects in the test group were administered systemic doxycycline } \\
\text { ( } 200 \mathrm{mg} \text { as loading dose and } 100 \mathrm{mg} \text { for } 20 \text { days), while patients in the control } \\
\text { group were administered placebo with the same instructions" }\end{array}$ \\
\hline
\end{tabular}


Tsalikis 2014 (Continued)

$\begin{aligned} & \text { Blinding of clinical opera- Low risk } \\ & \text { tor }\end{aligned} \quad \begin{aligned} & \text { Quote: "Neither therapists nor the examiner were aware of the treatment } \\ & \text { group" }\end{aligned}$

High risk

Incomplete outcome data

(attrition bias)

Per-protocol analysis: not all participants analysed in groups randomised to,

All outcomes

regardless of intervention actually received

4 patients (all Gp A) lost to follow-up. 2 at 3 months, and the remaining 2 at 6 months. All 4 cited as unwilling to continue

$\begin{array}{ll}\begin{array}{l}\text { Selective reporting (re- } \\ \text { porting bias) }\end{array} & \text { Low risk }\end{array}$

Other bias Unclear risk Study does not report proportion of patients in receipt of hypoglycaemic medications either by group or overall

Location: China

Setting: Hospital

Number of centres: 1, periodontal department of Guanghua College of Stomatology, Sun Yat-sen University, China

Recruitment period: Not reported

Funding source: Not reported

Participants

Inclusion criteria: Patients with newly diagnosed type 2 diabetes and no history of another major illness, no antibiotics or other medications received for at least 3 previous months; at least 14 standing teeth, pocket probing depth was $\geq 5 \mathrm{~mm}$, but $<8 \mathrm{~mm}$ in at least 1 site in 4 teeth in at least 2 different quadrants; bleeding and/or suppuration on probing; no periodontal treatment for 6 months prior to baseline examination

Exclusion criteria: Pregnancy or lactation

Age at baseline: Gp A mean 53.41 (SD 2.42) years, Gp B mean 55.10 (SD 2.64) years

Sex (M:F): Overall: M22:F24; Gp A: M10:F13; Gp B: M12:F11

Tobacco use: Not reported

Alcohol consumption: Not reported

Diabetes type: T2 DM

Duration since diabetes diagnosis: "newly diagnosed"

Metabolic control: Fair mean HbA1c at baseline

Mean $\mathrm{HbA1c}$ at baseline:Gp A 8.26\% (SD 0.31); Gp B 8.22\% (SD 0.45)

Antidiabetic therapy: Not specifically reported.

Quote: "These groups were well matched for ..oral hypoglycaemic medication, the proportion of patients prescribed diet control"

Other medical conditions: No history of other major illness

Number randomised: 46 
Yun 2007 (Continued)

\section{Number evaluated: 46}

\section{Interventions Comparison:SRP + OHI + doxycycline versus doxycycline alone}

Gp A ( $n=23)$ : Patients were treated weekly with 5 1-hour sessions on a weekly basis. First session OHI and supragingival scaling and polishing, then on subsequent sessions $\mathrm{OHI}$ reinforced and SRP under topical anaesthesia on quadrant by quadrant basis. Doxycycline $100 \mathrm{mg} /$ day for 14 days. Reassessed 8 weeks last session (3 months post-baseline)

Gp B ( $n=23)$ : Doxycycline $100 \mathrm{mg} /$ day for 14 days. This group received periodontal treatment as above after the end of the study

Duration of follow-up: 4 months

\begin{tabular}{ll}
\hline Outcomes & Primary: HbA1c (at baseline and 4 months) \\
& Secondary: BOP, PPD, CAL, PI (at baseline and 4 months) \\
\hline Notes & Sample size calculation: Not reported \\
& Data analysis: ITT \\
& Adverse events: Not reported \\
& Conflict of interests: Not reported \\
& SES: Not reported \\
HbA1c assessment method: High pressure liquid chromatography
\end{tabular}

Risk of bias

\begin{tabular}{|c|c|c|}
\hline Bias & Authors' judgement & Support for judgement \\
\hline $\begin{array}{l}\text { Random sequence genera- } \\
\text { tion (selection bias) }\end{array}$ & Unclear risk & Quote: "randomly divided" \\
\hline $\begin{array}{l}\text { Allocation concealment } \\
\text { (selection bias) }\end{array}$ & Unclear risk & Not reported \\
\hline Blinding of participants & High risk & Patients would know which group they were in \\
\hline $\begin{array}{l}\text { Blinding of clinical opera- } \\
\text { tor }\end{array}$ & Unclear risk & Not reported \\
\hline $\begin{array}{l}\text { Incomplete outcome data } \\
\text { (attrition bias) } \\
\text { All outcomes }\end{array}$ & Low risk & Not reported, but there do not seem to be any drop-outs. ITT analysis \\
\hline $\begin{array}{l}\text { Selective reporting (re- } \\
\text { porting bias) }\end{array}$ & Unclear risk & All measured outcomes were reported, except adverse events \\
\hline Other bias & High risk & Poorly reported \\
\hline
\end{tabular}

Zhang 2013

Methods

Trial design: 2 -arm, single-centre, parallel-design RCT

Location: China 
Zhang 2013 (Continued)

\author{
Setting: Hospital \\ Number of centres: 1 , Hubei Provincial Govt Hospital, Hubei, China \\ Recruitment period: July 2010 to May 2011 \\ Funding source: $11^{\text {th }}$ 5-year National Science and Technology Support Project (2007BAl18B02)
}

Participants

Inclusion criteria: Chronic periodontitis and had been diagnosed to have T2 DM for more than 1 year. A diagnosis of T2 DM should meet at least 1 of the following criteria: (1) postprandial plasma glucose $200 \mathrm{mg} / \mathrm{dL}$ (11.1 mmol/L); (2) fast plasma glucose (FPG) $126 \mathrm{mg} / \mathrm{dL}$ (7.0 mmol/L); (3) 2-hour oral glucose tolerance test $200 \mathrm{mg} / \mathrm{dL}$ (11.1 mmol/L). In addition, patients should have the following attributes: 35 to 80 years old; with at least 16 natural teeth; with at least 4 teeth with PPD $=5 \mathrm{~mm}, C A L=4 \mathrm{~mm}$, and $\mathrm{BOP}$, distributed in 2 or more oral quadrants; and the $\mathrm{HbA1c}$ level within 3 months before recruitment should at least be $5.5 \%$

Exclusion criteria: Accompanied with other systemic immune diseases; administered with antibiotics, immunomodulators, contraceptives, or any other form of hormone within the past 3 months; underwent modified diabetes treatment strategy within 3 months; had periodontal treatment within the past 12 months; needed extraction or endodontic treatment; smokes more than 4 cigarettes per day; pregnant or lactating women. Patients were dropped from the study if these conditions were met during the study course: diabetes treatment scheme was changed; drugs were systemically administered; patients could not revisit on time; participants were lost on follow-up

Age at baseline: Gp A mean 60.4 yrs (SD 9.77); Gp B mean 62.7 yrs (SD 10.7) ( $P=0.377)$

Sex (M:F): Overall: M31:F40; Gp A: M21:F28; Gp B: M10:F12 (P=0.838)

Tobacco use: Overall: $n=18(25 \%) ;$ Gp A: $n=12(24 \%) ; G p$ B: $n=6(27 \%)$

Alcohol consumption: Overall: $\mathrm{n}=20(28 \%) ; \mathrm{Gp} \mathrm{A}: \mathrm{n}=13(27 \%)$; $\mathrm{Gp}$ B: $\mathrm{n}=7$ (32\%)

Diabetes type: $\mathrm{T} 2 \mathrm{DM}$

Duration since diabetes diagnosis: Gp A 8.63 yrs (SD 4.20); Gp B 7.29 yrs (SD 5.61) $(P=0.305)$

Metabolic control: Fair mean $\mathrm{HbAlc}$ at baseline

Mean HbA1c at baseline: Gp A 7.68\% (SD 1.22); Gp B 7.38 (SD 1.30)

Antidiabetic therapy: All in receipt of oral hypoglycaemic medication, insulin or combination Overall: oral medication $n=55(77 \%)$; insulin $n=41(58 \%)$; Gp A: oral medication $n=40(82 \%)$; insulin $n$ $=30(61 \%) ;$ Gp B: oral medication $n=15(68 \%)$; insulin $n=11(50 \%)$

\title{
Other medical conditions: $\mathrm{n} / \mathrm{a}$
}

Other clinical investigations: FPG

Number randomised: $75 ; \mathrm{Gp} \mathrm{A} \mathrm{n}=50 ; \mathrm{Gp} \mathrm{B} n=25$

Number evaluated: 3 months $n=72 ; 6$ months $n=71$

Gp B $(\mathrm{n}=25)$ : Delayed treatment

Gp A subdivided at 3 months into Gp C ( $n=25 ; \mathrm{SRP}+\mathrm{OHI}+$ "sub-enhanced root planing" ("sub-ERP")) and Gp D = 25; SRP + OHI + "subprophylaxis" - HbA1c not reported with this further breakdown)

Duration of follow-up: 6 months 
Secondary: BOP, PPD, CAL, PI (at baseline, 3 and 6 months)

Notes Sample size calculation: Preliminary trial on 5 subjects per group SRP versus no treatment. A priori calculation at $80 \%$ power 20 in control and 40 in treatment group at $95 \%$ significance

Data analysis: Per-protocol

Adverse events: Not reported

Conflict of interests: Not reported

SES: Not reported

HbA1c assessment method: Ion exchange chromatography (Drew Scientific DS5, England)

\section{Risk of bias}

\begin{tabular}{|c|c|c|}
\hline Bias & Authors' judgement & Support for judgement \\
\hline $\begin{array}{l}\text { Random sequence genera- } \\
\text { tion (selection bias) }\end{array}$ & Unclear risk & $\begin{array}{l}\text { Pre-prepared randomisation in group A, B and C. No description of sequence } \\
\text { generation }\end{array}$ \\
\hline $\begin{array}{l}\text { Allocation concealment } \\
\text { (selection bias) }\end{array}$ & Low risk & Number coded-envelopes \\
\hline Blinding of participants & High risk & Not possible \\
\hline $\begin{array}{l}\text { Blinding of clinical opera- } \\
\text { tor }\end{array}$ & High risk & Not possible \\
\hline \multirow{2}{*}{$\begin{array}{l}\text { Incomplete outcome data } \\
\text { (attrition bias) } \\
\text { All outcomes }\end{array}$} & High risk & $\begin{array}{l}4 \text { lost to follow-up: Gp A: } 1 \text { lost at evaluation } 2 \text { ( } 3 \text { months); Gp B: } 2 \text { lost at evalu- } \\
\text { ation } 2 \text { ( } 3 \text { months), and } 1 \text { at evaluation } 3 \text { ( } 6 \text { months) }\end{array}$ \\
\hline & & $\begin{array}{l}\text { Per-protocol analysis: not all participants analysed in groups randomised to, } \\
\text { regardless of intervention actually received }\end{array}$ \\
\hline $\begin{array}{l}\text { Selective reporting (re- } \\
\text { porting bias) }\end{array}$ & High risk & $\begin{array}{l}\text { HbA1c data presented inconsistently, adverse effects not reported, periodon- } \\
\text { tal outcomes presented as graphs without data. Email to authors bounced }\end{array}$ \\
\hline Other bias & Low risk & No other apparent biases \\
\hline
\end{tabular}

aPDT = antimicrobial photodynamic therapy; $\mathrm{AL}=$ attachment level; $\mathrm{BI}=$ bleeding index; bid = twice daily; $\mathrm{BMI}=$ body mass index; $\mathrm{BOP}=$ bleeding on probing; $\mathrm{CAL}=$ clinical attachment level; $\mathrm{CHX}=$ chlorhexidine; $\mathrm{DM}=$ diabetes mellitus; $\mathrm{F}=$ female; $\mathrm{FMD}=$ full mouth disinfection; $\mathrm{GI}=$ gingival index; $\mathrm{Gp}$ = group; $\mathrm{GR}=$ gingival recession; ITT = intention-to-treat; $\mathrm{M}$ = male; NSAIDS = non-steroidal anti-inflammatory drugs; $\mathrm{OHI}=$ oral hygiene instruction; $\mathrm{PD}=$ probing depth; $\mathrm{PI}=$ plaque index; $\mathrm{po}=$ orally; $\mathrm{PPD}$ = probing pocket depth; qid = 4 times a day; $\mathrm{RCT}=$ randomised controlled trial; $\mathrm{SD}$ = standard deviation; $\mathrm{SE}$ = standard error; $\mathrm{SES}$ = socioeconomic status; SRP = scaling and root planing

Characteristics of excluded studies [ordered by study ID]

\begin{tabular}{ll}
\hline Study & Reason for exclusion \\
\hline Al-Mubarak 2002 & $\begin{array}{l}\text { Intervention (Waterpik, an irrigation device) is neither professionally-delivered, nor a periodontal } \\
\text { therapy }\end{array}$ \\
\hline Albrecht 1988 & $\begin{array}{l}\text { No HbAlc outcome reported. Study was not translated to English but advice sought from a Hungar- } \\
\text { ian speaker on the content }\end{array}$ \\
\hline Cinar 2014 & Intervention (empowerment "health coaching") is not a periodontal therapy \\
\hline \hline
\end{tabular}




\begin{tabular}{ll}
\hline Study & Reason for exclusion \\
\hline Gorbacheva 2010 & $\begin{array}{l}\text { Intervention (emoxypine succinate salt toothpaste) is neither professionally-delivered, nor a peri- } \\
\text { odontal therapy. Comparator also a toothpaste }\end{array}$ \\
\hline Hagiwara 2002 & $\begin{array}{l}\text { Study previously noted under ongoing studies (2010 version of this review); however, upon rein- } \\
\text { spection of abstract it clearly states non-diabetic control participants were included }\end{array}$ \\
\hline Khader 2010 & $\begin{array}{l}\text { Use of a non-periodontal intervention: full-mouth tooth extraction for patients whose remaining } \\
\text { teeth were deemed to be in a hopeless condition and indicated for extraction regardless }\end{array}$ \\
\hline Llambés 2012 & $\begin{array}{l}\text { No HbA1c outcome measurement (only hs-CRP), and appears to be subset of included Llambés } \\
\text { 2008 study (this second paper reporting hs-CRP separately from HbAlc data already reported in }\end{array}$ \\
\hline Llambés 2008)
\end{tabular}

Characteristics of studies awaiting assessment [ordered by study ID]

\section{Al-Mubarak 2010}

\begin{tabular}{ll}
\hline Methods & RCT conducted in Saudi Arabia \\
\hline Participants & 369 participants (T1DM and T2DM) randomised to 4 groups \\
& 346 participants completed (T1DM: $\mathrm{n}=33 ;$ T2DM: $\mathrm{n}=313)$ \\
\hline
\end{tabular}

Interventions

\section{Comparison: SRP + antimicrobial (doxycycline) versus SRP + placebo}

Gp 1: 1 x SRP session (baseline visit) + placebo tablets twice/day (at baseline for 3 months)

Gp 2: 1 x SRP session (baseline visit) + doxycycline hyclate (20 mg twice/day, at baseline for 3 months)

Gp 3: 2 x SRP session (baseline; 6 months) + placebo tablets twice/day (at baseline for 3 months; at 6-month visit for 3 months)

GP 4: 2 x SRP session (baseline; 6 months) + doxycycline hyclate $20 \mathrm{mg}$ twice/day (at baseline for 3 months; at 6-month visit for 3 months)

\begin{tabular}{ll}
\hline Outcomes & Primary: $\mathrm{HbAlC}$ at $3,6,9$ and 12 months \\
& Secondary: BOP, CAL, GI, PI, PPD at 3, 6, 9 and 12 months \\
& Duration: 12 months
\end{tabular}

Notes

International Standard Randomized Controlled Trial Number: ISRCTN-11742127 
Botero 2013

\begin{tabular}{ll}
\hline Methods & RCT conducted in Columbia \\
\hline Participants & 105 patients (T1DM $(n=39)$ or T2DM $(n=63))$ with moderate periodontitis \\
\hline
\end{tabular}

Interventions Comparison: Subgingival prophylaxis + antimicrobial (azithromycin) versus supragingival
prophylaxis + antimicrobial (azithromycin) versus subgingival prophylaxis + placebo

Gp A: Azithromycin $500 \mathrm{mg} /$ day $\times 3$ days + subgingival scaling

$\mathrm{Gp}$ B: Placebo $500 \mathrm{mg} /$ day $\times 3$ days + subgingival scaling

Gp C: Azithromycin $500 \mathrm{mg} /$ day $\times 3$ days + supragingival prophylaxis

\begin{tabular}{ll}
\hline Outcomes & Primary: HbAlc at 3, 6 and 9 months \\
& Secondary: CAL, PPD at 3, 6 and 9 months \\
& Duration: 9 months \\
\hline Notes & $\begin{array}{l}\text { Poorly reported. Need further data (particularly need accurate HbA1c means/SDs, data re: statin } \\
\text { use) from author to complete assessment. Awaiting reply from authors }\end{array}$ \\
\hline
\end{tabular}

Chee 2006

\begin{tabular}{ll}
\hline Methods & RCT conducted in Singapore \\
\hline Participants & 159 diabetic patients \\
\hline
\end{tabular}

\begin{tabular}{ll}
\hline Interventions & Comparison: SRP + OHI versus OHI versus no treatment \\
GP A: SRP + OHI \\
Gp B: OHI \\
Gp C: No treatment \\
\hline Outcomes & Primary: HbAlc (no indication of time points for recording) \\
& Secondary: BOP, PI, PPD (no indication of time points for recording) \\
\hline Notes & $\begin{array}{l}\text { No indication whether patients had diagnosed periodontitis. Poorly reported. Insufficient data to } 9 \text { months } \\
\text { complete assessment. Several attempts to contact authors for further details proved unsuccessful }\end{array}$ \\
\hline
\end{tabular}

Lin 2012

\begin{tabular}{ll}
\hline Methods & RCT conducted in Taiwan \\
\hline Participants & 28 T2DM patients \\
\hline
\end{tabular}


Lin 2012 (Continued)

Interventions

\section{Comparison: SRP + antimicrobial (minocycline) versus SRP}

Gp A: SRP (weekly quadrants undertaken over a month) + subgingival application of $2 \%$ minocycline gel

Gp B: SRP (weekly quadrants undertaken over a month)

\begin{tabular}{ll}
\hline Outcomes & Primary: HbAlc at 3, 6 months \\
& Secondary: BOP, CAL at 3, 6 months \\
& Duration: 6 months \\
\hline Notes & $\begin{array}{l}\text { No affirmation of diagnosed periodontitis. Also needed HbAlc data from author, but not provided } \\
\text { in email response. Emailed again to request, but no further response received }\end{array}$ \\
\hline
\end{tabular}

Nassar 2014

\begin{tabular}{|c|c|}
\hline Methods & RCT conducted in Brazil \\
\hline Participants & 40 (80? inconsistent reporting) diabetic patients \\
\hline Interventions & 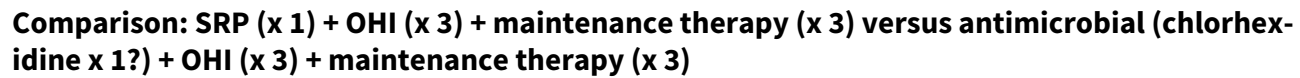 \\
\hline
\end{tabular}

Gp A: SRP (at baseline) + OHI (x 3: baseline, 3 months, 6 months) + mechanical maintenance therapy ((small-headed, soft-bristled toothbrush + standard toothpaste) $\times 3$ : baseline, 3 months, 6 months)

Gp B: Antimicrobial (chlorhexidine) full-mouth disinfection (at baseline) + $\mathrm{OHI}$ ( $\times$ 3: baseline, 3 months, 6 months) + mechanical maintenance therapy ((small-headed, soft-bristled toothbrush + standard toothpaste) $\times 3$ : baseline, 3 months, 6 months)

Primary: HbA1c at 3, 6 months
Secondary: BOP, CAL, PPD at 3, 6 months
Duration: 6 months

Notes

Poorly reported, particular clarification sought re: number of patients/randomisation/confirmed use of chlorhexidine (assumed from text). Awaiting response from authors

$\mathrm{BOP}=$ bleeding on probing; $\mathrm{CAL}=$ clinical attachment level; $\mathrm{GI}=$ gingival index; $\mathrm{Gp}=$ group $\mathrm{OHI}=$ oral hygiene instruction; $\mathrm{PI}=$ plaque index; $\mathrm{PPD}=$ probing pocket depth; RCT = randomised controlled trial; SD = standard deviation; SRP = scaling and root planing; T1DM = type 1 diabetes mellitus; T2DM = type 2 diabetes mellitus

Characteristics of ongoing studies [ordered by study ID]

ACTRN12605000260628

Trial name or title Assessment of diabetes after periodontal treatment

Trial acronym: ADAPT

\begin{tabular}{ll}
\hline Methods & RCT \\
\hline Participants & 60 patients
\end{tabular}

Treatment of periodontal disease for glycaemic control in people with diabetes mellitus (Review) 
Inclusion criteria: Either sex; $>35$ years old; able to give informed consent; random glucose $>200$ $\mathrm{mg} / \mathrm{dL}$; at least 16 teeth; chronic periodontitis

Exclusion criteria: Pregnancy; gross dental caries; requirement for antibiotic cover for dental treatment; anticoagulant therapy; other serious illness

Interventions $\begin{aligned} & \text { Comparison: SRP + antimicrobial toothpaste (triclosan) versus no treatment + placebo tooth } \\ & \text { paste }\end{aligned}$

Gp A: "Periodontal treatment" + triclosan/fluoride toothpaste

Gp B: Triclosan/fluoride toothpaste + delayed "periodontal treatment"

Follow-up duration: 12 months

\begin{tabular}{ll}
\hline Outcomes & $\begin{array}{l}\text { Primary: HbAlc (recorded at baseline, } 6 \text { and } 12 \text { months) } \\
\text { Secondary: (Unspecified: assume periodontal parameters) "response to periodontal treatmen- } \\
\text { t" (recorded at baseline, } 6 \text { and } 12 \text { months) }\end{array}$ \\
\hline Starting date & 2005 \\
\hline Contact information & Mary Cullinan: m.cullinan@uq.edu.au \\
\hline Notes & Funding source: Australian Dental Research Fund and Colgate Palmolive Co USA \\
& $\begin{array}{l}\text { Dr Cullinan confirmed (February 2015), completed but not published. Not able to share results (un- } \\
\text { known if analysed) }\end{array}$ \\
\hline
\end{tabular}

\begin{tabular}{|c|c|}
\hline Trial name or title & RMI adult oral health programme \\
\hline Methods & $\mathrm{RCT}$ \\
\hline \multirow[t]{3}{*}{ Participants } & 60 patients \\
\hline & $\begin{array}{l}\text { Inclusion criteria: Either sex; }>35 \text { years old; able to give informed consent; random glucose }>200 \\
\mathrm{mg} / \mathrm{dL} \text {; at least } 16 \text { teeth; chronic periodontitis }\end{array}$ \\
\hline & $\begin{array}{l}\text { Exclusion criteria: Pregnancy; requirement for antibiotic cover for dental treatment; anticoagulant } \\
\text { therapy; other serious illness }\end{array}$ \\
\hline \multirow[t]{4}{*}{ Interventions } & $\begin{array}{l}\text { Comparison: SRP + OHI + antimicrobial toothpaste (triclosan) versus SRP + OHI + placebo } \\
\text { toothpaste }\end{array}$ \\
\hline & $\mathrm{Gp} \mathrm{A:SRP}+\mathrm{OHI}+$ triclosan/fluoride toothpaste \\
\hline & Gp B: SRP + OHI + fluoride toothpaste (placebo) \\
\hline & Follow-up duration: 12 months \\
\hline \multirow[t]{2}{*}{ Outcomes } & Primary: HbA1c (recorded at baseline, 6 and 12 months) \\
\hline & Secondary: (Unspecified) "periodontal parameters" (recorded at baseline, 6 and 12 months) \\
\hline Starting date & 2005 \\
\hline Contact information & Mary Cullinan: m.cullinan@uq.edu.au \\
\hline Notes & Funding source: Colgate Palmolive Co USA \\
\hline
\end{tabular}


ACTRN12605000340639 (Continued)

Dr Cullinan confirmed (February 2015), completed but not published. Not able to share results (unknown if analysed)

\section{IRCT2014082417587N7}

Trial name or title

abetes mellitus

\begin{tabular}{ll}
\hline Methods & RCT \\
\hline
\end{tabular}

Participants 24 type 2 DM patients with chronic-moderate periodontitis

Interventions

Comparison: SRP + OHI + antimicrobial (doxycycline) versus SRP + OHI + placebo antimicrobial

Gp A: SRP + OHI + antimicrobial (doxycycline: $100 \mathrm{mg} /$ day $\times 15$ days)

Gp B: SRP + OHI + placebo antimicrobial (x 15 days)

Follow-up duration: 3 months

\begin{tabular}{ll}
\hline Outcomes & Primary: HbA1c (at 3 months) \\
& Secondary: BOP, CAL, PI, PPD (at 3 months) \\
\hline Starting date & September 2015 \\
\hline Contact information & Amirhossein Farahmand: perio-implant@hotmail.com \\
\hline Notes & Recruitment complete (September 2015) \\
\hline
\end{tabular}

\section{ISRCTN15334496}

Trial name or title Periodontal treatment for improving glycaemic control in diabetic patients: a randomised controlled trial

Trial acronym: DIAPERIO

Trial ID: ISRCTN15334496

Methods 2-centre, single-blind RCT

\section{Participants}

150 people with type 1 and 2 DM and:

- be aged 18 years or older (male or female),

- be affiliated to a public health system,

- be diagnosed as having had type 1 or 2 diabetes for at least 1 year before V0,

- have a last known value of HbA1c, measured within 3 months prior V0, between $6.8 \%$ and $9.7 \%$,

- have received stable antidiabetic therapy (no changes to diet, medication, dosage or formula-

tion) during the 3 months preceding Vo,

- have at least 6 natural permanent teeth,

- be available for all study visits over 3 months in the dental care departments (V1 to V6),

- be able to give their written informed consent

\section{Comparison: SRP + OHI + systemic antimicrobial (amoxicillin) + topical antimicrobial} (chlorhexidine) versus no treatment 
ISRCTN15334496 (Continued)

Gp A: SRP + OHI + systemic antimicrobial (500 mg amoxicillin tid for 7 days; or if contraindicated to beta-lactam antibiotics: $300 \mathrm{mg}$ clindamycin bid for 7 days) + topical antimicrobial (chlorhexidine $0.12 \%$ )

Gp B: No treatment (delayed until 23 weeks, when receive Gp A's allocated treatment)

\begin{tabular}{ll}
\hline Outcomes & $\begin{array}{l}\text { Primary: HbA1c after } 13 \text { weeks } \\
\text { Secondary: Quality of life } \\
\text { Duration: } 13 \text { weeks }\end{array}$ \\
\hline Starting date & May 2009 \\
\hline Contact information & Jean-Noel Vergnes: jn.vergnes@mcgill.ca \\
\hline Notes & Protocol only \\
& May 2013, email from JN Vergnes confirmed that this trial is still recruiting \\
& Followed up December 2014, no response from author
\end{tabular}

\section{ISRCTN83229304}

Trial name or title Periodontitis and type 2 diabetes mellitus

Trial acronym: DRN211

\begin{tabular}{ll}
\hline Methods & Single centre RCT \\
\hline Participants & 280 patients \\
& Inclusion criteria: Either sex; $>18$ years old; able to give consent; diagnosed type 2 DM; at least 15 \\
& teeth; signs of active moderate-severe periodontitis ( 20 periodontal pockets, PPD $>4$ mm and \\
& BOP) \\
& Exclusion criteria: Pregnancy; requirement for antibiotic cover for periodontal procedures; chronic \\
& treatment with phenytoin/cyclosporin; known HIV/hepatitis B or C/uncontrolled systemic disease; \\
& neoplasm
\end{tabular}

Interventions

\section{Comparison: "Intensive periodontal therapy" versus supragingival scaling and polishing}

Gp A: "Intensive periodontal therapy"

Gp B: "Usual periodontal care (supragingival scaling and polishing)"

Follow-up duration: 12 months

\begin{tabular}{ll}
\hline Outcomes & Primary: HbAlc (recorded at baseline and 12 months) \\
\hline Starting date & 2008 \\
\hline Contact information & Jean Suvan: j.suvan@eastman.ucl.ac.uk \\
\hline Notes & Author confirmed (December 2014) data currently being analysed \\
\hline
\end{tabular}


NCT00016835

\begin{tabular}{|c|c|}
\hline Trial name or title & Treating periodontal infection: effects on glycaemic control in people with type 2 diabetes \\
\hline Methods & 3-arm RCT \\
\hline Participants & 45 type 2 DM patients with established periodontitis \\
\hline \multirow[t]{4}{*}{ Interventions } & $\begin{array}{l}\text { Comparison: Scaling + topical antimicrobial (povidone-iodine) + systemic antimicrobial } \\
\text { (doxycycline) versus scaling + topical antimicrobial (povidone-iodine) + systemic antimicro- } \\
\text { bial (metronidazole) versus supragingival prophylaxis/calculus removal + placebo + delayed } \\
\text { subgingival scaling + topical antimicrobial (povidone-iodine) }\end{array}$ \\
\hline & $\begin{array}{l}\text { Gp A: Ultrasonic scaling with local anaesthesia (as needed), local antimicrobial treatment with } \\
\text { povidone-iodine irrigation and an oral systemic antibiotic (doxycycline) at baseline }\end{array}$ \\
\hline & $\begin{array}{l}\text { Gp B: Ultrasonic scaling with local anaesthesia (as needed), local antimicrobial treatment with } \\
\text { povidone-iodine irrigation and an oral systemic antibiotic (metronidazole) at baseline }\end{array}$ \\
\hline & $\begin{array}{l}\text { Gp C: Placebo and supragingival oral prophylaxis and ultrasonic removal of supragingival calculus } \\
\text { with water irrigation at baseline, + subgingival ultrasonic scaling with povidone-iodine irrigation at } \\
9 \text { months }\end{array}$ \\
\hline \multirow[t]{2}{*}{ Outcomes } & Primary: HbA1c. Others unknown \\
\hline & Duration: 15 months \\
\hline Starting date & April 2001 \\
\hline Contact information & George Taylor: gwt@umich.edu \\
\hline Notes & $\begin{array}{l}\text { Author (GT) previously supplied some information for } 2010 \text { review indicating that data had been } \\
\text { collected and was awaiting analysis. Emailed Dr Taylor to check if trial completed/obtain unpub- } \\
\text { lished results, but no response }\end{array}$ \\
\hline
\end{tabular}

\begin{tabular}{ll}
\hline Trial name or title & Periodontal treatment and metabolic control in type 2 diabetic patients \\
\hline Methods & 2-arm RCT \\
\hline Participants & 732 type 2 DM patients with severe periodontitis \\
\hline Interventions & Comparison: SRP versus mechanical debridement \\
& $\begin{array}{l}\text { Gp A: SRP under local analgesia (depending on the severity in 1 session or 2 sessions within 2 days) } \\
+ \text { extraction of indicated hopeless teeth + additional SRP where necessary at follow-up } \\
\text { Gp B: "Supragingival biofilm control": supragingival mechanical instrumentation/polishing using } \\
\text { hand and machine driven (piezoelectric) instrumentation }\end{array}$ \\
\hline Outcomes & Primary: HbA1c (at 2, 6, 12 months). Others unknown \\
\hline Starting date & February 2011 \\
\hline Contact information & Hilana Artese: hilanartese@gmail.com; Giuseppe Romito: garomito@usp.br \\
\hline
\end{tabular}


NCT01291875 (Continued)

Notes

Emailed Drs Artese and Romito to check if trial completed/obtain unpublished results, but no response

NCT01881074

\begin{tabular}{|c|c|}
\hline Trial name or title & Periodontal treatment response in type II diabetic patients \\
\hline Methods & 2-arm RCT \\
\hline Participants & 68 type 2 DM patients \\
\hline \multirow[t]{3}{*}{ Interventions } & $\begin{array}{l}\text { Comparison: Prophylaxis }(\times 6)+\text { antimicrobial toothpaste (triclosan) versus prophylaxis }(\times 6) \\
\text { + placebo toothpaste }\end{array}$ \\
\hline & $\begin{array}{l}\text { Gp A: "Dental cleaning" ( } 6 \text { : at baseline, } 6 \text { weeks, 3, 6, 9, and } 12 \text { months) + use of provided antimi- } \\
\text { crobial toothpaste (triclosan) (for full } 12 \text { months) }\end{array}$ \\
\hline & $\begin{array}{l}\text { Gp B: "Dental cleaning" ( } \times 6 \text { : at baseline, } 6 \text { weeks, 3, 6, 9, and } 12 \text { months) + use of provided placebo } \\
\text { toothpaste (for full } 12 \text { months) }\end{array}$ \\
\hline \multirow[t]{3}{*}{ Outcomes } & Primary: HbA1c (at 3, 6, 12 months) \\
\hline & Secondary: CAL, PI, PPD (at 3, 6, 12 months) \\
\hline & Duration: 12 months \\
\hline Starting date & June 2012 \\
\hline Contact information & Luciana Shaddox: ShaddoxResearch@dental.ufl.edu \\
\hline \multirow[t]{3}{*}{ Notes } & Emailed Dr Shaddox to check if trial completed/obtain unpublished results, but no response \\
\hline & Collaborative study with Colgate-Palmolive \\
\hline & No indication of patients requiring diagnosis of periodontitis \\
\hline
\end{tabular}

\section{NCT01901926}

\begin{tabular}{ll}
\hline Trial name or title & Periodontal treatment and glycaemic control \\
\hline Methods & RCT \\
\hline Participants & 184 type 2 DM patients with mild-moderate periodontitis
\end{tabular}

Interventions Comparison: SRP versus no treatment

Gp A: SRP

Gp B: No treatment

\begin{tabular}{ll}
\hline Outcomes & Primary: $\operatorname{HbA1c}$ (at 3, 6, 9 months) \\
& Secondary: BOP, CAL, PPD (at 3, 6, 9 months) \\
& Duration: 9 months
\end{tabular}


NCT01901926 (Continued)

Starting date December 2012

\section{Contact information}

Notes
Salman Aziz:dr_salman_aziz@yahoo.com

Emailed Dr Aziz to check if trial completed/obtain unpublished results, but no response

\section{NCT01904422}

\begin{tabular}{|c|c|}
\hline Trial name or title & Periodontal treatment in non-controlled type 2 diabetes mellitus patients (FONIS12I2106) \\
\hline Methods & 2-arm RCT \\
\hline Participants & 100 type 2 DM patients \\
\hline \multirow[t]{3}{*}{ Interventions } & $\begin{array}{l}\text { Comparison: Immediate treatment (SRP + additional mechanical therapy (prophylaxis) + OHI) } \\
\text { versus ongoing treatment SRP + additional mechanical therapy (prophylaxis) + OHI) }\end{array}$ \\
\hline & $\begin{array}{l}\text { Gp A: Intensive treatment ( } 2 \text { sessions (left side, then right) in } 24 \text { hrs: SRP + OHI + supragingival and } \\
\text { subgingival debridement) }\end{array}$ \\
\hline & $\begin{array}{l}\text { Gp B: Ongoing treatment ( } 5 \text { sessions: } 1 \text { quadrant } \mathrm{p} / \mathrm{w} \text { ) over } 5 \text { weeks: SRP }+\mathrm{OHI}+\text { supragingival and } \\
\text { subgingival debridement) }\end{array}$ \\
\hline \multirow[t]{3}{*}{ Outcomes } & Primary: HbA1c (at 3, 6 months) \\
\hline & Secondary: BOP, CAL, PPD (at 3, 6 months) \\
\hline & Duration: 6 months \\
\hline Starting date & March 2013 \\
\hline Contact information & Helia Morales: heliamorales@gmail.com \\
\hline \multirow[t]{2}{*}{ Notes } & No indication of patients requiring diagnosis of periodontitis \\
\hline & Emailed Dr Morales to check if trial completed/obtain unpublished results, but no response \\
\hline
\end{tabular}

\section{NCT01964833}

Trial name or title

Combination of photodynamic therapy and periodontal treatment in patients with type 2 diabetes mellitus: randomised, double-blind clinical trial

Trial acronym: PDTDMT2

\begin{tabular}{ll}
\hline Methods & RCT \\
\hline Participants & 44 patients \\
& Inclusion criteria: Either sex; >18 years old; compensated type 2 DM or with adequate control based \\
on the criteria of the Brazilian Society of Diabetes (SBD, 2012); chronic periodontitis (AAP, 2001); at \\
least 15 teeth and at least 4 teeth with BOP and PPD >4 mm; under follow-up with an endocrinolo- \\
gist \\
Exclusion criteria: Uncompensated diabetes, based on SBD criteria; smoking habit in 12 months \\
prior to treatment; anaemia; active cancer; current pregnancy; history of antibiotic therapy in pre-
\end{tabular}


NCT01964833 (Continued)

vious 6 months; history of anti-inflammatory therapy in previous 3 months; clotting disorder (use of anti-coagulant, presence of liver disease, thrombocytopenia and immunosuppression); currently undergoing orthodontic treatment

\begin{tabular}{|c|c|}
\hline Interventions & $\begin{array}{l}\text { Comparison: SRP + OHI + aPDT versus SRP + OHI + placebo aPDT } \\
\text { Gp A: SRP + OHI + aPDT } \\
\text { Gp B: SRP + OHI + placebo aPDT } \\
\text { Follow-up duration: } 6 \text { months }\end{array}$ \\
\hline Starting date & 2013 \\
\hline Notes & $\begin{array}{l}\text { Author reports (December } 2014 \text { ) trial neither completed nor published, and encountered signifi- } \\
\text { cant problems with following-up patients }\end{array}$ \\
\hline
\end{tabular}

U1111-1124-3635

\begin{tabular}{ll}
\hline Trial name or title & Influence of periodontal treatment in periodontitis and diabetes control \\
\hline Methods & RCT \\
\hline Participants & 150 type 2 DM patients with chronic periodontitis \\
\hline
\end{tabular}

Interventions Comparison: SRP versus ultrasonic debridement versus OHI

Gp A: SRP

Gp B: Ultrasonic debridement

Gp C: OHI

\begin{tabular}{ll}
\hline Outcomes & Primary: HbAlc \\
& Secondary: PI, PPD \\
& Duration: 6 months \\
\hline Starting date & August 2011 \\
\hline Contact information & Renata Cimões: renata.cimoes@globo.com \\
\hline Notes & Emailed to check if completed/unpublished results available: no response \\
\hline
\end{tabular}

aPDT = antimicrobial photodynamic therapy; bid = twice daily; $\mathrm{BOP}=$ bleeding on probing; $\mathrm{CAL}=\mathrm{clinical}$ attachment level; $\mathrm{DM}=$ diabetes mellitus; $\mathrm{Gp}=$ group; $\mathrm{OHI}=$ oral hygiene instruction; $\mathrm{PI}=$ plaque index; $\mathrm{PPD}=$ probing pocket depth; RCT = randomised controlled trial; SRP = scaling and root planing; tid = 3 times a day 


\section{DATA AND ANALYSES}

Comparison 1. Periodontal therapy versus no active intervention/usual care

\begin{tabular}{|c|c|c|c|c|}
\hline $\begin{array}{l}\text { Outcome or sub- } \\
\text { group title }\end{array}$ & No. of studies & $\begin{array}{l}\text { No. of partici- } \\
\text { pants }\end{array}$ & Statistical method & Effect size \\
\hline $1 \mathrm{HbA} 1 \mathrm{c}$ at $3-4$ months & 14 & 1499 & Mean Difference (IV, Random, 95\% CI) & $-0.29[-0.48,-0.10]$ \\
\hline $1.1 \mathrm{SRP}$ & 8 & 547 & Mean Difference (IV, Random, 95\% CI) & $-0.41[-0.73,-0.08]$ \\
\hline $\begin{array}{l}\text { 1.2 SRP + antimicro- } \\
\text { bials }\end{array}$ & 7 & 952 & Mean Difference (IV, Random, 95\% CI) & $-0.18[-0.39,0.03]$ \\
\hline $2 \mathrm{HbA} 1 \mathrm{c}$ at 6 months & 5 & 826 & Mean Difference (IV, Random, 95\% CI) & $-0.02[-0.20,0.16]$ \\
\hline $2.1 \mathrm{SRP}$ & 3 & 263 & Mean Difference (IV, Random, 95\% CI) & $-0.18[-0.58,0.22]$ \\
\hline $\begin{array}{l}2.2 \text { SRP + antimicro- } \\
\text { bials }\end{array}$ & 2 & 563 & Mean Difference (IV, Random, 95\% CI) & $0.02[-0.18,0.22]$ \\
\hline
\end{tabular}

Analysis 1.1. Comparison 1 Periodontal therapy versus no active intervention/usual care, Outcome 1 HbA1c at 3-4 months.

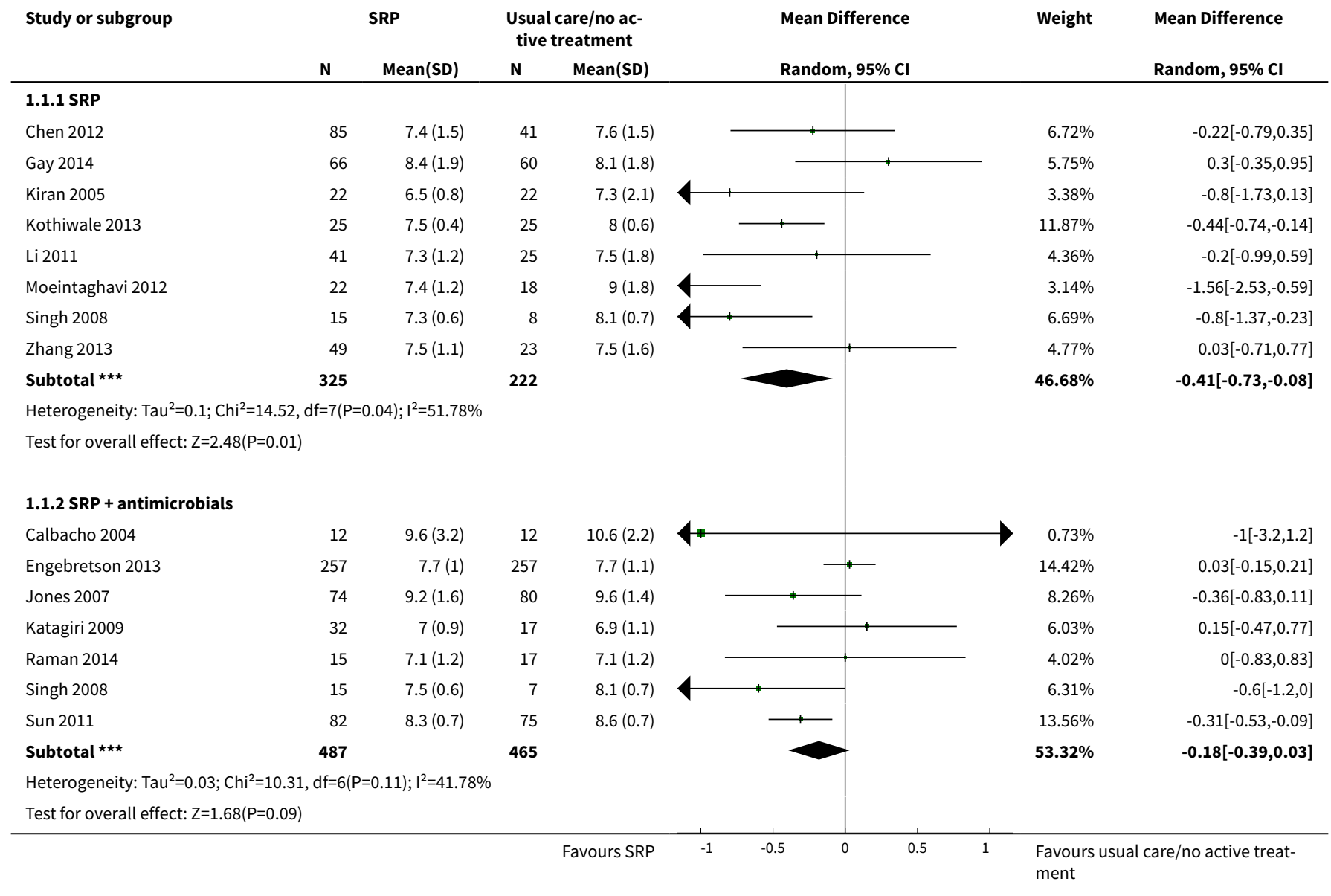




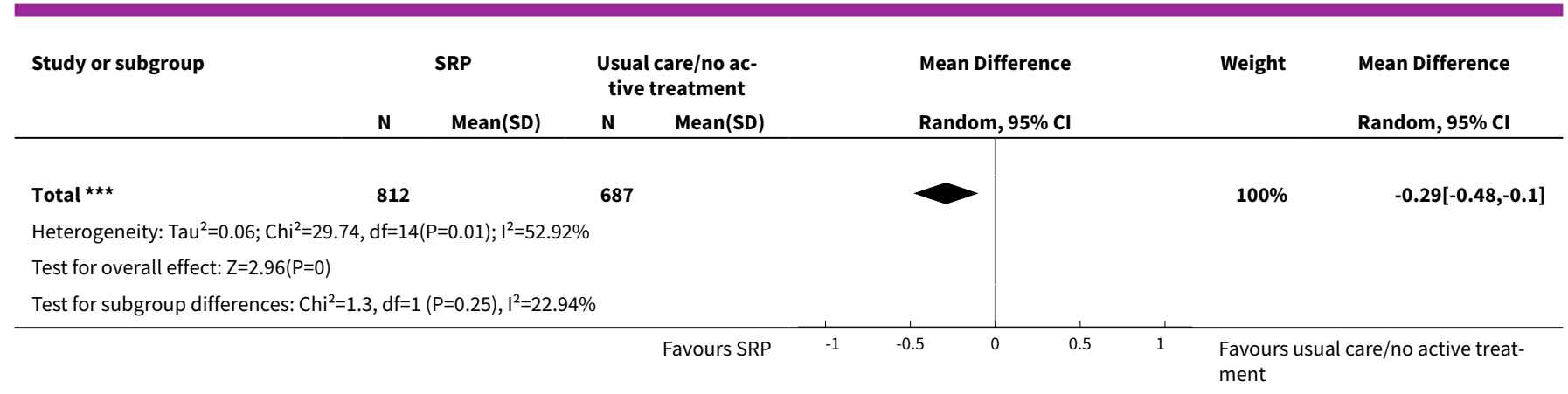

Analysis 1.2. Comparison 1 Periodontal therapy versus no

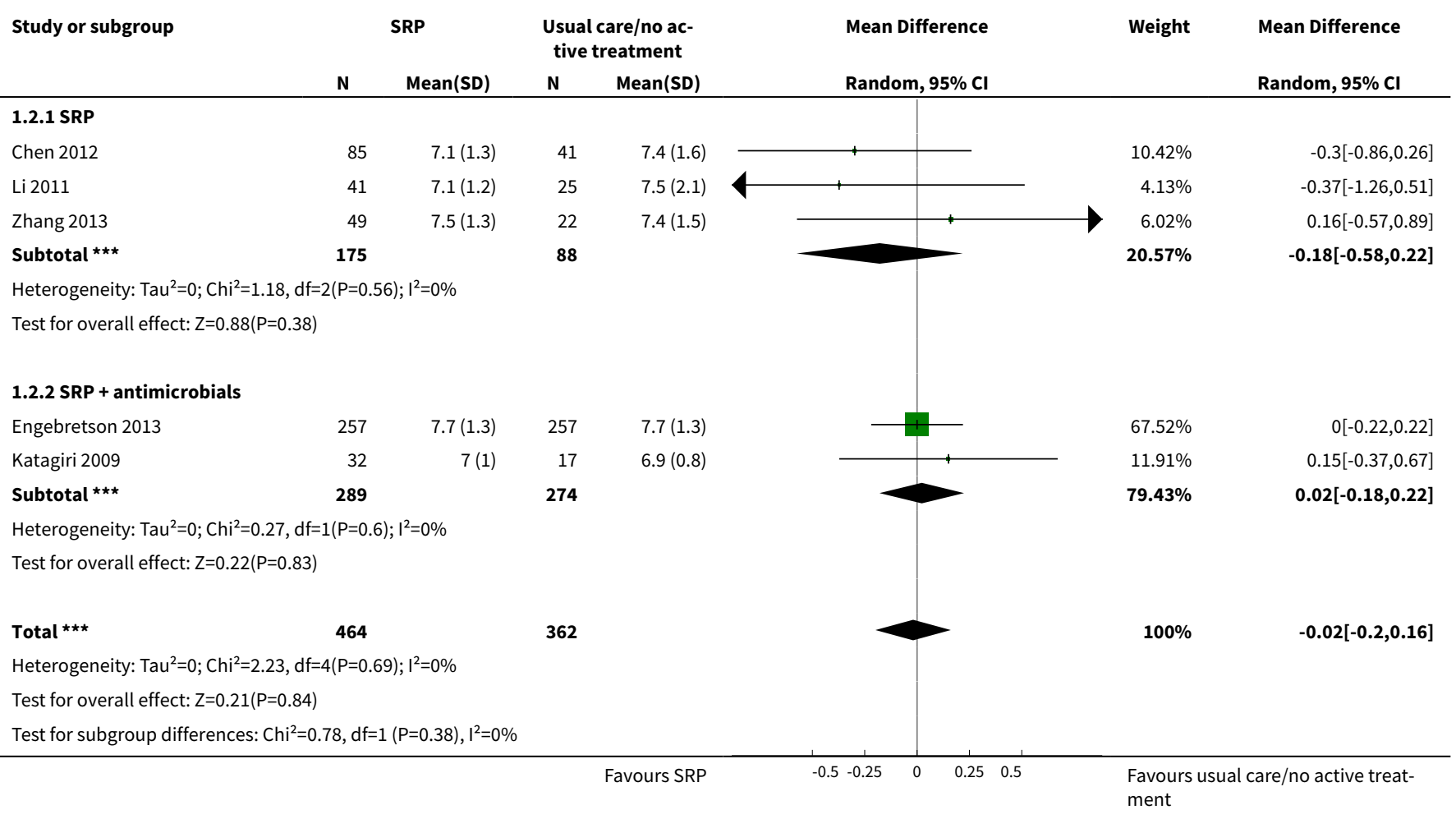

\section{Comparison 2. Periodontal therapy versus alternative periodontal therapy}

\begin{tabular}{|c|c|c|c|c|}
\hline Outcome or subgroup title & No. of studies & $\begin{array}{l}\text { No. of partici- } \\
\text { pants }\end{array}$ & Statistical method & Effect size \\
\hline $1 \mathrm{HbA} 1 \mathrm{c}$ at 3-4 months & 21 & & $\begin{array}{l}\text { Mean Difference (IV, Random, } \\
95 \% \mathrm{CI} \text { ) }\end{array}$ & $\begin{array}{l}\text { Totals not select- } \\
\text { ed }\end{array}$ \\
\hline $\begin{array}{l}1.1 \text { SRP vs alternative mechanical } \\
\text { therapy }\end{array}$ & 1 & & $\begin{array}{l}\text { Mean Difference (IV, Random, } \\
95 \% \mathrm{CI} \text { ) }\end{array}$ & $0.0[0.0,0.0]$ \\
\hline 1.2 SRP vs alternative SRP & 3 & & $\begin{array}{l}\text { Mean Difference (IV, Random, } \\
95 \% \mathrm{CI} \text { ) }\end{array}$ & $0.0[0.0,0.0]$ \\
\hline
\end{tabular}




\begin{tabular}{|c|c|c|c|c|}
\hline Outcome or subgroup title & No. of studies & $\begin{array}{l}\text { No. of partici- } \\
\text { pants }\end{array}$ & Statistical method & Effect size \\
\hline $\begin{array}{l}\text { 1.3 SRP + antimicrobial vs antimi- } \\
\text { crobial }\end{array}$ & 1 & & $\begin{array}{l}\text { Mean Difference (IV, Random, } \\
95 \% \mathrm{CI} \text { ) }\end{array}$ & $0.0[0.0,0.0]$ \\
\hline 1.4 SRP + antimicrobial vs SRP & 12 & & $\begin{array}{l}\text { Mean Difference (IV, Random, } \\
95 \% \mathrm{CI})\end{array}$ & $0.0[0.0,0.0]$ \\
\hline $\begin{array}{l}1.5 \text { SRP + antimicrobial (doxycy- } \\
\text { cline) vs SRP + alternative antimi- } \\
\text { crobial }\end{array}$ & 3 & & $\begin{array}{l}\text { Mean Difference (IV, Random, } \\
95 \% \mathrm{CI})\end{array}$ & $0.0[0.0,0.0]$ \\
\hline $\begin{array}{l}\text { 1.6 SRP + combined antimicrobials } \\
\text { vs SRP + single antimicrobial }\end{array}$ & 3 & & $\begin{array}{l}\text { Mean Difference (IV, Random, } \\
95 \% \mathrm{CI})\end{array}$ & $0.0[0.0,0.0]$ \\
\hline 1.7 SRP + statin vs SRP & 1 & & $\begin{array}{l}\text { Mean Difference (IV, Random, } \\
95 \% \mathrm{CI})\end{array}$ & $0.0[0.0,0.0]$ \\
\hline $2 \mathrm{HbA} 1 \mathrm{c}$ at 6 months & 12 & & $\begin{array}{l}\text { Mean Difference (IV, Random, } \\
95 \% \mathrm{CI})\end{array}$ & $\begin{array}{l}\text { Totals not select- } \\
\text { ed }\end{array}$ \\
\hline $\begin{array}{l}2.1 \text { SRP vs alternative mechanical } \\
\text { therapy }\end{array}$ & 1 & & $\begin{array}{l}\text { Mean Difference (IV, Random, } \\
95 \% \mathrm{CI})\end{array}$ & $0.0[0.0,0.0]$ \\
\hline 2.2 SRP vs alternative SRP & 3 & & $\begin{array}{l}\text { Mean Difference (IV, Random, } \\
95 \% \mathrm{CI} \text { ) }\end{array}$ & $0.0[0.0,0.0]$ \\
\hline 2.3 SRP + antimicrobial vs SRP & 5 & & $\begin{array}{l}\text { Mean Difference (IV, Random, } \\
95 \% \mathrm{CI} \text { ) }\end{array}$ & $0.0[0.0,0.0]$ \\
\hline $\begin{array}{l}2.4 \text { SRP + antimicrobial (doxycy- } \\
\text { cline) vs SRP + alternative antimi- } \\
\text { crobial }\end{array}$ & 1 & & $\begin{array}{l}\text { Mean Difference (IV, Random, } \\
95 \% \mathrm{CI})\end{array}$ & $0.0[0.0,0.0]$ \\
\hline $\begin{array}{l}2.5 \mathrm{SRP}+\text { combined antimicrobials } \\
\text { vs SRP + single antimicrobial }\end{array}$ & 1 & & $\begin{array}{l}\text { Mean Difference (IV, Random, } \\
95 \% \mathrm{CI})\end{array}$ & $0.0[0.0,0.0]$ \\
\hline 2.6 SRP + bone modifier vs SRP & 1 & & $\begin{array}{l}\text { Mean Difference (IV, Random, } \\
95 \% \mathrm{CI} \text { ) }\end{array}$ & $0.0[0.0,0.0]$ \\
\hline 2.7 SRP + statin vs SRP & 1 & & $\begin{array}{l}\text { Mean Difference (IV, Random, } \\
95 \% \mathrm{CI} \text { ) }\end{array}$ & $0.0[0.0,0.0]$ \\
\hline
\end{tabular}

Analysis 2.1. Comparison 2 Periodontal therapy versus alternative periodontal therapy, Outcome $1 \mathrm{HbA1C}$ at 3-4 months.

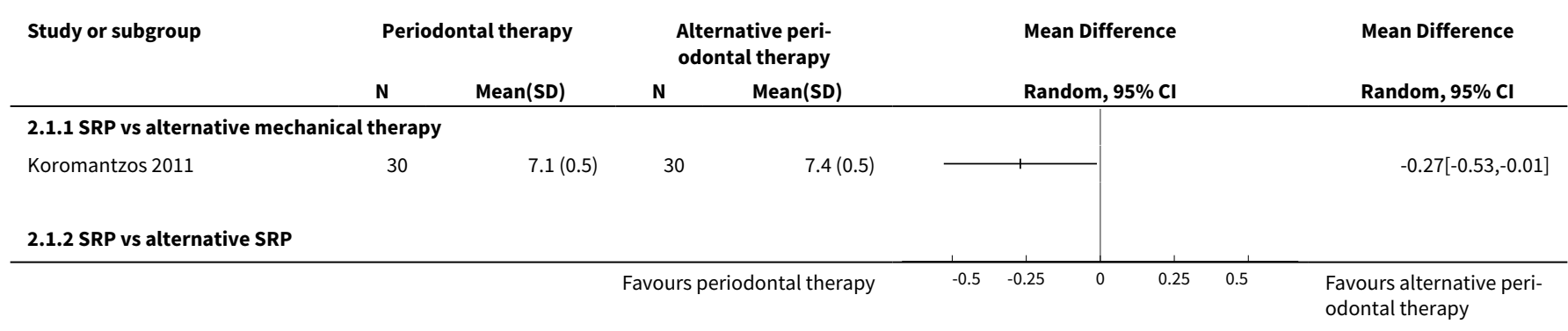




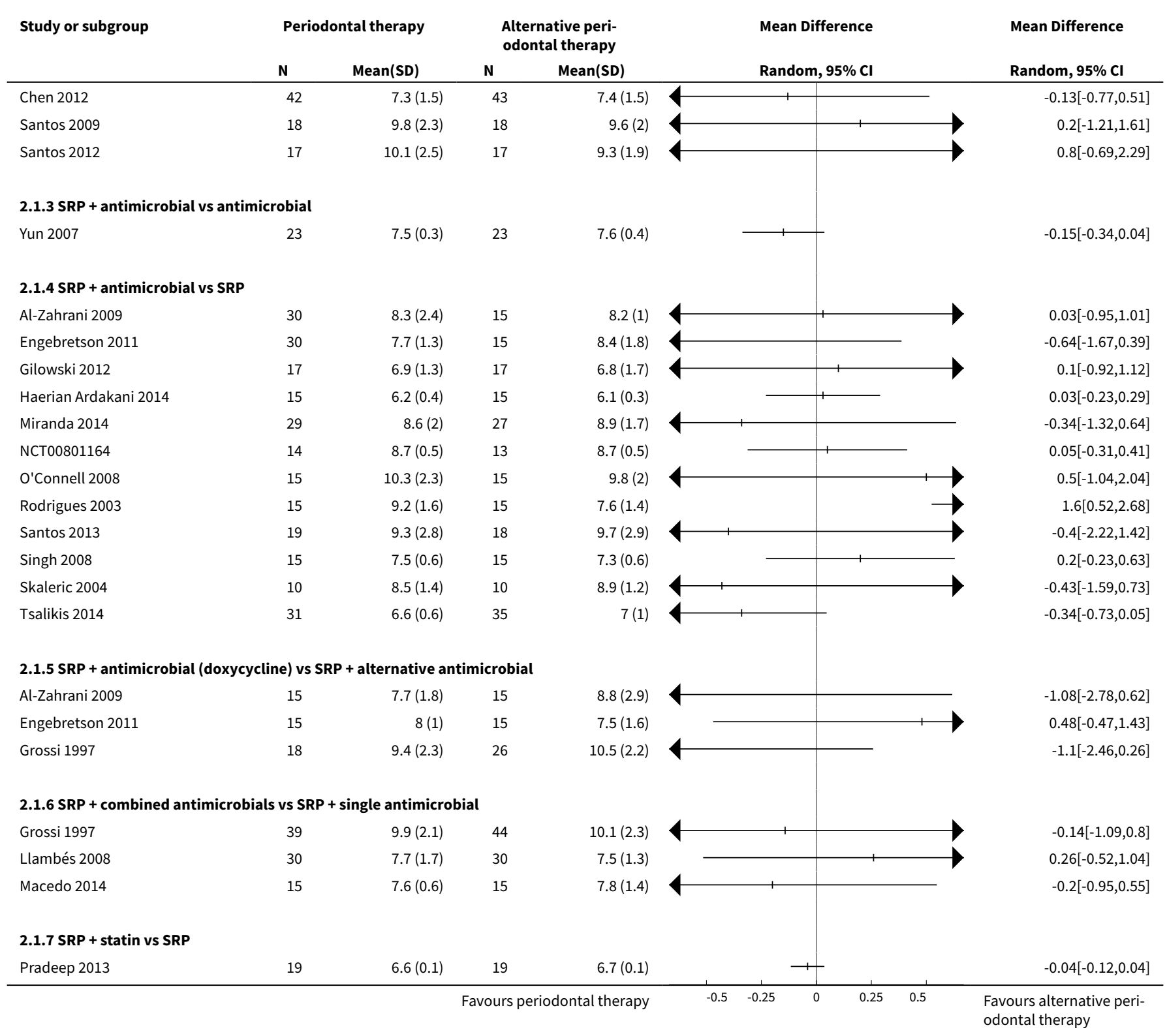

Analysis 2.2. Comparison 2 Periodontal therapy versus alternative periodontal therapy, Outcome $2 \mathrm{HbA1C}$ at 6 months.

\begin{tabular}{|c|c|c|c|c|c|c|c|c|c|}
\hline \multirow[t]{2}{*}{ Study or subgroup } & \multicolumn{2}{|c|}{ Periodontal therapy } & \multicolumn{2}{|c|}{$\begin{array}{l}\text { Alternative peri- } \\
\text { odontal therapy }\end{array}$} & \multirow{2}{*}{\multicolumn{4}{|c|}{$\begin{array}{l}\text { Mean Difference } \\
\text { Random, } 95 \% \mathrm{CI}\end{array}$}} & \multirow{2}{*}{$\begin{array}{l}\text { Mean Difference } \\
\text { Random, } 95 \% \mathrm{Cl}\end{array}$} \\
\hline & $\mathbf{N}$ & Mean(SD) & $\mathbf{N}$ & Mean(SD) & & & & & \\
\hline \multicolumn{10}{|c|}{ 2.2.1 SRP vs alternative mechanical therapy } \\
\hline Koromantzos 2011 & 30 & $7.2(0.7)$ & 30 & $7.5(0.7)$ & & & - & & $-0.31[-0.67,0.05]$ \\
\hline \multicolumn{10}{|c|}{ 2.2.2 SRP vs alternative SRP } \\
\hline Chen 2012 & 42 & $7.1(1.3)$ & 43 & $6.9(1.1)$ & & & & & $0.22[-0.31,0.75]$ \\
\hline Santos 2009 & 18 & $9.5(1.9)$ & 18 & $10.3(2.6)$ & & & & & $-0.8[-2.29,0.69]$ \\
\hline \multirow[t]{2}{*}{ Santos 2012} & 17 & $9.7(2)$ & 17 & $10.2(2.9)$ & & & & & $-0.5[-2.17,1.17]$ \\
\hline & & & Favours & dontal therapy & -0.5 & -0.25 & 0.25 & 0.5 & $\begin{array}{l}\text { Favours alternative peri- } \\
\text { odontal therapy }\end{array}$ \\
\hline
\end{tabular}




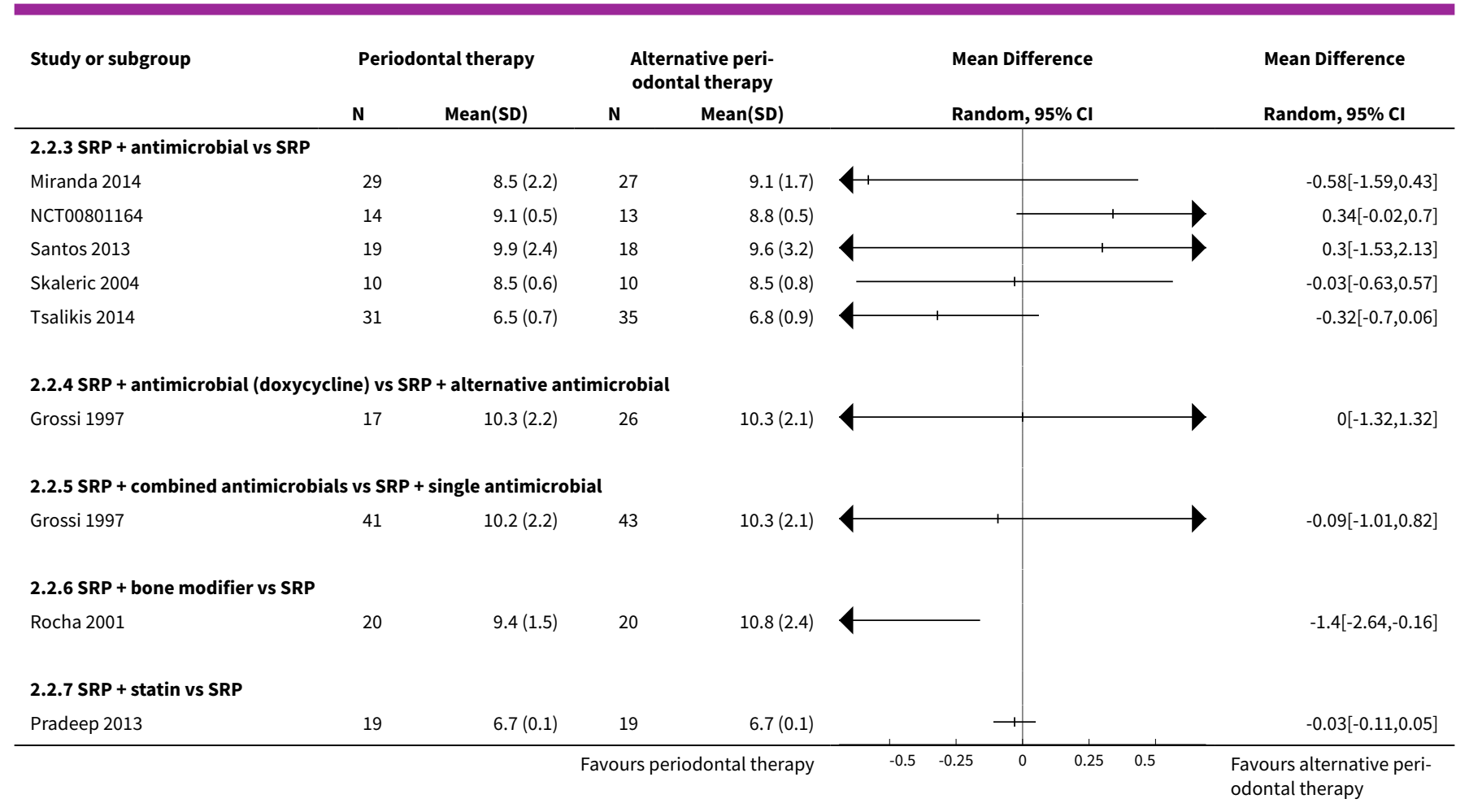

ADDITIONAL TABLES

Table 1. Diagnostic criteria (diabetes mellitus and periodontal disease)

\begin{tabular}{|c|c|c|}
\hline Study & $\begin{array}{l}\text { Diabetes assessment of patients for inclu- } \\
\text { sion }\end{array}$ & Periodontitis assessment of patients for inclusion \\
\hline Al-Zahrani 2009 & Diagnosed with Type 2 DM & CAL: $\geq 3 \mathrm{~mm}$ at $\geq 30 \%$ of sites \\
\hline Calbacho 2005 & Diagnosed with Type 2 DM & $\begin{array}{l}\text { Quote: "moderate chronic marginal periodontitis diagno- } \\
\text { sis" }\end{array}$ \\
\hline Chen 2012 & Diagnosed with Type 2 DM for $>1$ year & $\begin{array}{l}\text { American Academy of Periodontology criteria, with } a \geq 1 \\
\mathrm{~mm} \text { mean CAL }\end{array}$ \\
\hline Engebretson 2011 & $\begin{array}{l}\text { Diagnosed with Type } 2 \text { DM at least } 6 \text { months } \\
\text { previously }\end{array}$ & $\mathrm{CAL}>5 \mathrm{~mm}$ in at least 1 site in each jaw quadrant \\
\hline Engebretson 2013 & $\begin{array}{l}\text { Diagnosed with Type } 2 \mathrm{DM} \text { more than } 3 \\
\text { months duration, an } \mathrm{HbA} 1 \mathrm{c} \text { value between } \\
7.0 \% \text { and less than } 9.0 \% \text { at screening }\end{array}$ & $\begin{array}{l}\text { CAL and PPD of at least } 5 \mathrm{~mm} \text { in } 2 \text { or more quadrants of } \\
\text { the mouth }\end{array}$ \\
\hline Gay 2014 & $\begin{array}{l}\text { Diagnosed with Type } 2 \text { DM. HbA1c levels } \\
\geq 6.5 \% \text {; initial } \mathrm{HbA} 1 \mathrm{c} \text { values between } 5.7 \%- \\
6.5 \% \text { were included if they were taking hy- } \\
\text { poglycaemic medications ( } \mathrm{n}=16 \text { ) }\end{array}$ & $\begin{array}{l}\text { Severe chronic periodontitis according to American Acad- } \\
\text { emy of Periodontology criteria }\end{array}$ \\
\hline Gilowski 2012 & Diagnosed with Type 2 DM & At least 4 non-adjacent sites with $P D \geq 4 \mathrm{~mm}$ \\
\hline
\end{tabular}


Table 1. Diagnostic criteria (diabetes mellitus and periodontal disease) (Continued)

\begin{tabular}{|c|c|c|}
\hline Grossi 1997 & WHO criteria for designation as having DM & $\begin{array}{l}\text { No periodontal inclusion criteria stated; mean PPD } \\
\text { around } 3.5 \text { to } 3.7 \mathrm{~mm} \text { for all groups; CAL in the range } 4.5 \mathrm{t} \\
5 \mathrm{~mm} \text { at baseline }\end{array}$ \\
\hline
\end{tabular}

\begin{tabular}{lll}
\hline Haerian Ardakani 2014 & Diagnosed with Type 2 DM, HbAlc $\leq 7 \%$ & $\begin{array}{l}\text { Chronic periodontitis with 3 regions probe depth }>4 \text { mm } \\
\text { and }<7 \mathrm{~mm}\end{array}$ \\
\hline Jones $\mathbf{2 0 0 7}$ & $\begin{array}{l}\text { Statement that inclusion depended on a re- } \\
\text { peat HbA1c of > or equal to 8.5\% }\end{array}$ & $\begin{array}{l}\text { Community Periodontal Index of Treatment Need (CPITN; } \\
\text { Ainamo 1982) scores of } \geq 3 \text { in at least 2 sextants }\end{array}$ \\
\hline Katagiri $\mathbf{2 0 0 9}$ & Type 2 DM and HbA1c 6.5\%-10.0\% & At least 2 pocket sites with PPD $\geq 4 \mathrm{~mm}$ \\
\hline
\end{tabular}

\begin{tabular}{lll}
\hline Kiran 2005 & $\begin{array}{l}\text { Diabetes - participants under treatment for } \\
\text { Type 2 DM with HbAlc in the range 6\%-8\% }\end{array}$ & Not reported \\
\hline Koromantzos 2011 & Type 2 DM and HbA1c levels from 7\% to 10\% & $\begin{array}{l}\text { At least 8 sites with PPD } \geq 6 \text { mm and 4 sites with CAL } \geq 5 \\
\mathrm{~mm} \text {, distributed in at least 2 different quadrants }\end{array}$ \\
\hline Kothiwale 2013 & $\begin{array}{l}\text { Type 2 DM with a minimum duration of 2 } \\
\text { years }\end{array}$ & $\begin{array}{l}\text { CPI (community periodontal index: PPD } \geq 4 \text { mm) and LA } \\
\text { (loss of attachment: CAL } \geq 4 \text { mm) indices (as stated in Pe- } \\
\text { ter 2007) }\end{array}$ \\
\hline
\end{tabular}

\begin{tabular}{lll}
\hline Li 2011 & Type 2 DM & Not reported \\
\hline Llambes 2008 & $\begin{array}{l}\text { Type 1 DM with severities defined by the } \\
\text { American Diabetes Association criteria }\end{array}$ & At least 5 teeth with a site of PPD $\geq 5$ mm and CAL $\geq 3$ mm \\
\hline Macedo 2014 & $\begin{array}{l}\text { Type 2 DM diagnosed for }>5 \text { years and HbA1c } \\
>7 \%\end{array}$ & $\begin{array}{l}\text { At least } 1 \text { site with PPD } \geq 5 \text { mm on each quadrant, and 2 } \\
\text { teeth with CAL } \geq 6 \text { mm }\end{array}$ \\
\hline Madden 2008 & $\begin{array}{l}\text { Type 2 DM for }>1 \text { year; HbA1c }>7 \% \text { but } \\
<13.11 \%\end{array}$ & Löe and Silness Gl \\
\hline Miranda 2014 & $\begin{array}{l}\text { Type 2 DM for } \geq 5 \text { years; HbA1c levels } \geq 6.5 \% \\
\leq 11 \%\end{array}$ & $\begin{array}{l}\text { More than 30\% of the sites with PPD and CAL } \geq 4 \text { mm and a } \\
\text { minimum of } 6 \text { teeth with at least } 1 \text { site with PPD and CAL } \\
\geq 5 \text { mm and BOP at baseline }\end{array}$ \\
\hline
\end{tabular}

\begin{tabular}{|c|c|c|}
\hline Moeintaghavi 2012 & $\begin{array}{l}\text { Diagnosis of type } 2 \text { DM with glycated } \\
\text { haemoglobin (HbA1c) values over } 7 \%\end{array}$ & American Academy of Periodontology criteria \\
\hline NCT00801164 & $\begin{array}{l}\text { Self reported type } 2 \text { DM of more than } 3 \\
\text { months duration; a current HbAlc value be- } \\
\text { tween } 7.0 \% \text { and } 12 \%\end{array}$ & $\begin{array}{l}\text { Moderate to severe chronic periodontitis, defined by loss } \\
\text { of clinical attachment of }>5 \mathrm{~mm} \text { on } 2 \text { separate teeth }\end{array}$ \\
\hline O'Connell 2008 & $\begin{array}{l}\text { Type } 2 \text { DM diagnosed for }>5 \text { years and } \mathrm{HbA} 1 \mathrm{C} \\
>8 \%\end{array}$ & At least 1 site with $P D \geq 5 \mathrm{~mm}$ and 2 teeth with $C A L \geq 6 \mathrm{~mm}$ \\
\hline Pradeep 2013 & $\begin{array}{l}\text { Classed as type } 2 \text { DM based on the American } \\
\text { Diabetic Association } 2011 \text { criteria }\end{array}$ & $\mathrm{PD} \geq 5 \mathrm{~mm}$ or $\mathrm{CAL} \geq 4 \mathrm{~mm}$ and vertical bone loss $\geq 3 \mathrm{~mm}$ \\
\hline Raman 2014 & $\begin{array}{l}\text { Type } 2 \text { DM diagnosed at least } 1 \text { year prior to } \\
\text { the study }\end{array}$ & $\begin{array}{l}\text { PD } 5 \text { or more pockets of } \geq 5 \mathrm{~mm} \text { and probing } \mathrm{AL} \text { of } \geq 4 \mathrm{~mm} \\
\text { or more in at least } 2 \text { different quadrants which bled on } \\
\text { probing }\end{array}$ \\
\hline Rocha 2001 & Type 2 DM for at least 5 years & PPD $>3 \mathrm{~mm}$ in at least 1 tooth \\
\hline Rodrigues 2003 & Diagnosed with Type 2 DM & 1 site and 2 teeth with $>5 \mathrm{~mm}$ PPD and $>6 \mathrm{~mm} \mathrm{CAL}$ \\
\hline
\end{tabular}


Table 1. Diagnostic criteria (diabetes mellitus and periodontal disease) (Continued)

$\begin{array}{lll}\text { Santos } 2009 & \text { Type } 2 \text { DM within the past } 5 \text { years } & \begin{array}{l}>30 \% \text { of sites with PD and CAL } \geq 5 \mathrm{~mm} \text { at baseline (based } \\ \text { on the 1999 World Workshop for classification of Peri- } \\ \text { odontal Diseases and Conditions) }\end{array}\end{array}$

\begin{tabular}{lll}
\hline Santos 2012 & Type 2 DM for at least the past 5 years & $>30 \%$ of sites with PD and CAL $\geq 4$ mm at baseline \\
\hline Santos 2013 & Type 2 DM for at least the past 5 years & $>30 \%$ of sites with PD and CAL $\geq 4$ mm at baseline \\
\hline Singh 2008 & Type 2 DM & $\geq 30 \%$ teeth PD and CAL $\geq 4$ mm at baseline \\
\hline Skaleric 2004 & Type 1 DM <5 years & 4 teeth in at least 2 quadrants with $\geq 5$ mm PD \\
\hline Sun 2011 & Type 2 DM for over a year; HbA1c: 7.5\%-9.5\% & $\begin{array}{l}>20 \text { teeth, probing depth }>5 \text { mm, more than } 30 \% \text { teeth } \\
\text { with attachment loss }>4 \text { mm, or over } 60 \% \text { teeth with PD }>4 \\
\text { mm and AL }>3 \text { mm }\end{array}$ \\
\hline
\end{tabular}

Tsalikis $2014 \quad$ Type $2 \mathrm{DM}$, diagnosed at least 1 year before 6 pockets $>5 \mathrm{~mm}$ and CAL $>3$ mm with radiographic bone baseline examination; at least 2 consecutive loss values of $\mathrm{HbA} 1 \mathrm{c}<7.5 \%$ as assessed by the patients' medical records

\begin{tabular}{ll}
\hline Yun 2007 Newly diagnosed Type 2 DM & $\begin{array}{l}\text { Periodontal - PPD > or equal to } 5 \mathrm{~mm} \text { but }<8 \mathrm{~mm} \text { in } 1 \text { site } \\
\text { in } 4 \text { teeth or } 2 \text { different quadrants. No indication of CAL or } \\
\text { alveolar bone loss }\end{array}$ \\
\hline
\end{tabular}

Zhang 2013 Type 2 DM for $>1$ year; HbA1c level within 3 months before recruitment should at least be $5.5 \%$
At least 4 teeth with $P P D \geq 5 \mathrm{~mm}, \mathrm{CAL} \geq 4 \mathrm{~mm}$, and BOP, distributed in 2 or more oral quadrants

Study authors' inclusion criteria for diabetes and periodontal disease

$\mathrm{AL}=$ attachment loss; $\mathrm{BOP}=$ bleeding on probing; $\mathrm{CAL}=$ clinical attachment level; $\mathrm{DM}=$ diabetes mellitus; $\mathrm{GI}=$ gingival index; $\mathrm{PD}=\mathrm{pocket}$ depth; $\mathrm{PPD}=$ probing pocket depth; $\mathrm{WHO}=$ World Health Organization

\section{Table 2. Diabetic control at baseline (HbA1c threshold for participants)}

- 4 studies (11\%) had the broadest defined inclusion thresholds ranging at least a $4 \%$ difference in HbA1c levels (Madden 2008 (fair-poor: 7.0\%-13.11\%); NCT00801164 (fair-poor: 7.0\%-12.0\%); Miranda 2014 (good-poor: 6.5\%-11.0\%); Katagiri 2009 (good-poor: $6.5 \%-10.0 \%))$

- 4 studies (11\%) ranged a defined 2\%-3\% difference in HbA1c levels (Kiran 2005 (good-fair: 6.0\%-8.0\%); Engebretson 2013 (fair-poor: 7.0\%-9.0\%); Sun 2011 (fair-poor: 7.5\%-9.5\%); Koromantzos 2011 (fair-poor: 7.0\%-10.0\%))

- 3 studies (8\%) only included participants within the threshold (good: $<7.5 \%$ ) for controlled diabetes (Gay 2014 (patients in receipt of diabetic treatment (79\%) - good: 5.7\%-6.5\%; patients not receiving diabetic treatment (21\%) - good-poor: >6.5\%); Haerian Ardakani 2014 (<7.0\%); Tsalikis $2014(<7.5 \%))$

- 1 study (3\%) included patients with HbAlc levels >5.5\% (good: Zhang 2013) without a defined upper threshold limit for inclusion

- 2 studies (8\%) included patients with HbA1c levels $>7.0 \%$ (fair-poor: Macedo 2014; Moeintaghavi 2012) without a defined upper threshold limit for inclusion

- 2 studies (6\%) only included patients with uncontrolled HbA1c levels (Jones 2007 (poor: >8.5\%); Skaleric 2004 (poor: >9.0\%)) without a defined upper threshold limit for inclusion 
Table 2. Diabetic control at baseline (HbA1c threshold for participants) (Continued)

- 19 trials (53\%) did not report their patient inclusion criteria for HbA1c levels (Al-Zahrani 2009; Calbacho 2004; Chen 2012; Engebretson 2011; Gilowski 2012; Grossi 1997; Kothiwale 2013; Li 2011; Llambés 2008; O'Connell 2008; Pradeep 2013; Raman 2014; Rocha 2001; Rodrigues 2003; Santos 2009; Santos 2012; Santos 2013; Singh 2008; Yun 2007)

\section{Table 3. Types of antidiabetic therapy in included trials}

- In five studies (17\%), all patients were reported to be in receipt of oral hypoglycaemic medication, insulin, controlled diet or combination treatment (Gilowski 2012; Kiran 2005; Santos 2009; Santos 2012; Santos 2013)

- All patients were in receipt of oral hypoglycaemic medication, insulin, or combination treatment (no diet component) in 3 studies (9\%) (Jones 2007; Koromantzos 2011; Li 2011)

- In Engebretson 2013 (3\%), all except 11 patients (2\% of 514 participants) were in receipt of oral hypoglycaemic medications, insulin, or combination treatment (no diet component)

- In 2 studies (6\%), all patients were in receipt of oral hypoglycaemic medication, insulin, or controlled diet (no combination treatment) (Chen 2012; Katagiri 2009)

- 2 trials' patients used oral hypoglycaemic medication or insulin (no controlled diet or combination therapy) (Al-Zahrani 2009; Engebretson 2011)

- In 1 study (3\%), all patients were in receipt of oral hypoglycaemic medication, insulin, or combination treatment (no diet component) (Zhang 2013)

- In 1 study, all patients used oral hypoglycaemic medication, but some also used insulin (no further details provided, except that insulin use was stratified to each group) (Grossi 1997)

- 4 studies' (11\%) patients only used oral hypoglycaemic medication to control their diabetes (Calbacho 2004; Kothiwale 2013; Miranda 2014; Moeintaghavi 2012)

- In 1 study (Llambés 2008), all patients solely used insulin to control their diabetes

- Singh 2008 only included patients in receipt of antidiabetic therapy but gave no indication what form

- Gay 2014 (3\%) only reports that all except 26 patients (21\% of 126 participants) were in receipt of "diabetic treatment" without further description

- 13 studies (39\%) did not report use of antidiabetic therapy use (Haerian Ardakani 2014; Macedo 2014; Madden 2008; NCT00801164; O'Connell 2008; Pradeep 2013; Raman 2014; Rocha 2001; Rodrigues 2003 (excluded insulin users, but no other detail); Skaleric 2004 (excluded non-insulin users, but no other detail); Sun 2011; Tsalikis 2014; Yun 2007 (only that groups were well matched for oral hypoglycaemic medication and diet control - no further detail))

Table 4. Changes in antidiabetic therapy during study period

\begin{tabular}{lll}
\hline Study ID & Pre-intervention & Change in diabetic therapy during study \\
\hline Al-Zahrani 2009 & $\begin{array}{l}\text { All (seemingly) in receipt of oral hypoglycaemic med- } \\
\text { ication (Overall: 72\%) or insulin (Overall: 28\%; Gp A: } \\
\text { 20\%; Gp B: 29\%; Gp C: } 36 \%(\mathrm{P}=0.64))\end{array}$ & $\begin{array}{l}\text { Quote: "None of the participants reported a } \\
\text { change in the types or doses of their medica- } \\
\text { tions during the study period" }\end{array}$ \\
\hline Calbacho $\mathbf{2 0 0 5}$ & All in receipt of oral hypoglycaemic medication only & $\begin{array}{l}\text { Authors report in correspondence that no varia- } \\
\text { tion of antidiabetic therapy occurred during the } \\
\text { study }\end{array}$ \\
\hline \hline
\end{tabular}


Table 4. Changes in antidiabetic therapy during study period (Continued)

Chen 2012

in receipt of oral hypoglycaemic medication (Gp A 38; Gp B: 35; Gp C: 36), insulin (Gp A: 4; Gp B: 5; Gp C: 4), or diet (Gp A: 0; Gp B: 3; Gp C: 1) (P= 0.574)

Not reported

Inclusion criteria required all patients to be in receipt of stable dosage of oral hypoglycaemic medications or insulin

Engebretson 2013

All except 11 patients ( $2 \%$ of 514 participants) were in receipt of oral hypoglycaemic medication (Overall: 47\%; Gp A: 46\%; Gp B: 49\%), insulin (Overall: 16\%; Gp A: $16 \%$; Gp B: $16 \%$ ), or combination treatment (Overall: 35\%; Gp A: 37\%; Gp B: 33\%)

Inclusion criteria required agreement to continue existing antidiabetic therapy unless medically indicated otherwise, and no changes to have been made to current therapy for prior 3 months
Gay $2014 \quad$ All except 26 patients ( $21 \%$ of 126 participants) were in receipt of "diabetic treatment" (Gp A: 79\%; Gp B: $80 \%$ ) without further description

Of diabetic treatment recipients, 21 patients were on insulin therapy: Gp A: $21 \%(n=14) ; G p ~ B: 12 \%(n=7)$
Study confirms use of medications remained constant during study period

Quote: "Of the 462 participants with medication data available at all study visits, 128 of 233 $(55 \%)$ in the treatment group and 137 of 229 $(60 \%)$ in the control group had no protocol-defined changes in diabetes medications during the study"
Quote: "About 18 test and 13 control subjects had changes in their medications during the 4month trial"

No indication whether these numbers reflect baseline patients (Gp A: 66; Gp B: 60) or analysed patients (Gp A: 48; Gp B: 42)

\section{Gilowski 2012}

Quote: "All patients received optimal diabetic treatment including diet regimen, insulin supplementation, and/or oral hypoglycaemic drugs"
Not reported
All in receipt of oral hypoglycaemic medication Also states groups were stratified by insulin use, but no further detail provided
Dose and type of medication monitored. Most changes were to other oral agents (not described in detail). 2 people each in 2 arms (Gp C, and Gp E) were changed to insulin therapy. Similar results found when people whose treatment had been changed were excluded (but actual data not given)

\begin{tabular}{ll} 
Haerian Ardakani 2014 & Not reported \\
\hline Jones 2007 & Pattern of treatment similar in both groups ( 2 2 = \\
& $\begin{array}{l}50.89, \mathrm{P}=0.64) \text { for proportions receiving insulin, in- } \\
\text { sulin and oral hypoglycaemic, oral hypoglycaemic } \\
\text { alone }\end{array}$
\end{tabular}

\section{Katagiri 2009}

All in receipt of oral hypoglycaemic medication, insulin, or diet

Diet: Overall: $\mathrm{n}=3$; Gp A: $\mathrm{n}=1 ; \mathrm{Gp} \mathrm{B}: \mathrm{n}=2$

Oral hypoglycaemic medication: Overall: $\mathrm{n}=27$; Gp A: $\mathrm{n}=15 ; \mathrm{Gp}$ B: $\mathrm{n}=12$

Insulin: Overall: $n=19 ; G p$ A: $n=16 ;$ Gp B: $n=3$
Not reported

Participants in the usual care group were twice as likely $(20 \%$ versus $11 \%, P<0.12)$ to increase insulin from baseline to 4 months and less likely to decrease insulin $(1 \%$ versus $6 \%, P<0.21)$

Quote: "The doses and kinds of anti-diabetic drugs, including oral hypoglycaemic drugs and insulin injections and methods of diet and exercise were not changed to assess the real effects of periodontal treatment on blood glucose control"

Quote: "No change in the medication or diet was made for both groups during the study period" A: 64\%; Gp B: 72\%), insulin (Gp A: 9\%; Gp B: 9\%), diet (Gp A: $9 \%$; Gp B: $5 \%$ ) or combination (Gp A: $18 \%$; Gp B:14\%) 
Table 4. Changes in antidiabetic therapy during study period (Continued)

Koromantzos 2011 All in receipt of oral hypoglycaemic medication, insulin or both

Insulin: Gp A n = 12 (40\%); Gp B n = 7 (23.3\%)

OHA: Gp A n = 21 (70\%); Gp B n = $27(90 \%)$
Quote: "Over the course of the study, no oral medication changes were performed, while a similar number of [Gp A] and [Gp B] participants increased their insulin dosages [four (13.3\%) for [Gp A] and three (10.0\%) for [Gp B] participants. Out of the seven patients that increased their insulin dosages, five (three in [Gp A] and two in [Gp B]) were patients that were lost to follow-up"

No changes reported, but also not anticipated due to protocol instruction not to change antidiabetic therapy during the course of the study abetes, diet and physical therapy was unchanged throughout the course of the study as monitored by the physician"

Kothiwale 2013

\section{Li 2011}

Gp A (oral hypoglycaemic agents: $77.3 \% /$ insulin injection: $27.3 \%)$; Gp B (78.9\%/21.1\%); Gp C (76\%/16\%)
Not identified by translation

Quote: "Insulin doses were quite stable in both groups. 18 patients from group 1 and 20 patients from group 2 did not change their insulin dose during the clinical investigation. 12 patients changed insulin doses in group 1 , but half of them had a variation of less than 3 units a day. In group 2, 10 patients modified insulin doses during the study, and 8 of them had changes of less than 3 units a day"

\begin{tabular}{lll}
\hline Macedo 2014 & Not reported & Not reported \\
\hline Madden 2008 & Not reported & $\begin{array}{l}\text { Quote: "Of the 42 remaining subjects who com- } \\
\text { pleted the study, 15 had their diabetes medica- } \\
\text { tions changed at the advice of their physicians } \\
\text { (Gp n=9; Gp B n=6). There were } 27 \text { subjects who } \\
\text { did not have diabetic medication changes" }\end{array}$ \\
\end{tabular}

Miranda 2014

Quote: "all subjects included in this study reported to be under metformin or glibenclamide treatment. In addition, two subjects per group also reported to be under insulin supplementation"
Not reported to inform investigators if any change occurred"
All in receipt of oral hypoglycaemic medications (no insulin)

Inclusion criteria specifies patients "blood sugar controlled with glybenclamide and metformin, without insulin administration"
Quote: "our study as a prerequisite included only patients who did not have any change in their diabetic control regimen during the 3-month study period"

No further detail, assumed no changes

Unknown due to study not yet having been published

Not reported

Not reported

Quote: "2 of the 5 subjects from [Gp A] who did not complete the study had their diabetic medication changed during the course of the study and had to be excluded...For [Gp B], 1 sub- 
ject had his medications for Type 2 diabetes changed"

\begin{tabular}{|c|c|c|}
\hline Rocha 2001 & Not reported & Not reported \\
\hline Rodrigues 2003 & $\begin{array}{l}\text { Insulin users excluded from participation, no other } \\
\text { detail reported }\end{array}$ & $\begin{array}{l}\text { Quote: "alterations in ... diabetes control were } \\
\text { recorded" - however, data not reported }\end{array}$ \\
\hline Santos 2009 & $\begin{array}{l}\text { All in receipt of oral hypoglycaemic medication, in- } \\
\text { sulin, diet or combination } \\
\text { Diet: Overall: } n=6 ; \text { Gp A: } n=2 ; \text { Gp B: } n=4 \\
\text { Diet + insulin: Overall: } n=5 ; \text { Gp A: } n=3 ; \text { Gp B: } n=2 \\
\text { Diet + OHA: Overall: } n=21 ; G p A: n=11 ; G p ~ B: n=10 \\
\text { Diet + OHA + insulin: Overall: } n=4 ; G p A: n=2 ; G p \text { B: } n \\
=2\end{array}$ & $\begin{array}{l}\text { Quote: "To assess the effects of the periodontal } \\
\text { treatments on metabolic control, no changes } \\
\text { in the medication or diet were made during the } \\
\text { study period ( } 6 \text { months)" }\end{array}$ \\
\hline
\end{tabular}

Santos $2012 \quad$ All in receipt of oral hypoglycaemic medication, insulin, diet or combination

Diet: Overall: $n=6 ; G p$ A: $n=3 ; G p$ B: $n=3$

Diet + insulin: Overall: $n=3 ; G p A: n=1 ; G p$ B: $n=2$

Diet + OHA: Overall: $n=23 ; G p A: n=12 ; G p ~ B: n=11$

Diet + OHA + insulin: Overall: $n=2 ; G p A: n=1 ; G p ~ B: n$

$=1$

\section{Santos 2013}

All in receipt of oral hypoglycaemic medication, insulin, diet or combination

Diet: Overall: $\mathrm{n}=1 ; \mathrm{Gp} A: \mathrm{n}=1 ; \mathrm{Gp}$ B: $\mathrm{n}=0$

Diet + insulin: Overall: $n=5 ; G p A: n=1 ; G p$ B: $n=4$

Diet + OHA: Overall: $n=28 ; G p$ A: $n=14 ; G p B: n=14$

Diet + OHA + insulin: Overall: $n=4 ; G p A: n=3 ; G p$ B: $n$

$=1$

\begin{tabular}{ll}
\hline Singh 2008 & $\begin{array}{l}\text { Exclusion criteria specifies "Patients with uncon- } \\
\text { trolled DM" }\end{array}$
\end{tabular}

Quote: "..we did not attempt to change the diabetic control of our patients by giving any additional instructions for control of blood glucose levels"

\section{Skaleric 2004}

Not specifically reported. "Patients with type 2 noninsulin dependent diabetes were excluded from the study"

insulin dependent diabetes were excluded from the
study"

Quote: "no changes in the category of treatment regimen for DM occurred during the study"
Quote: "subjects reported no changes in the category of DM treatment regimen during the study"
Quote: "No change in the medication or diet was made for the patients. None of the patients received any additional guidance for managing their diabetic status"

Quote: "Insulin dosage was adjusted according to the routine procedure for blood glucose self management.... made at the discretion of the diabetologist" - however, data not reported

\begin{tabular}{lll}
\hline Sun $\mathbf{2 0 1 1}$ & Not reported & Not reported \\
\hline Tsalikis 2014 & Not reported & Not reported \\
\hline Yun 2007 & $\begin{array}{l}\text { Not specifically reported. } \\
\text { Quote: "These groups were well matched for .., oral } \\
\text { hypoglycemic medication, the proportion of patients } \\
\text { prescribed diet control" }\end{array}$ & Not reported \\
\hline
\end{tabular}

\section{Zhang 2013}

All in receipt of oral hypoglycaemic medication, insulin or combination

Not reported

Overall: oral medication $n=55(77 \%)$; insulin $n=41$

(58\%); Gp A: oral medication $n=40$ (82\%); insulin $n=$ 
Table 4. Changes in antidiabetic therapy during study period (Continued) 30 (61\%); Gp B: oral medication $n=15(68 \%)$; insulin $n$ $=11(50 \%)$

Table 5. Periodontal therapies compared in included studies

\section{Comparison 1: Periodontal therapy vs no active inter- vention/usual care $(n=9)$}

Subgroup 1.1: SRP $(n=5)$

- $\mathrm{SRP}+\mathrm{OHI}+$ additional mechanical therapy vs no active intervention (Chen 2012 (3 of 3 arms: Gps A+B combined vs Gp C))

- SRP vs OHI (Gay 2014)

- SRP + OHI vs no active intervention (Kiran 2005; Kothiwale 2013; Singh 2008 (2 of 3 arms: Gp A vs Gp C); Zhang 2013)

- Periodontal therapy described as "mechanical therapy" vs OHI (Li 2011 (3 of 3 arms: Gps A+B combined vs Gp C))

- SRP vs no active intervention (Moeintaghavi 2012)

Subgroup 1.2: SRP + antimicrobials $(n=4)$

- SRP + antimicrobial (doxycycline) vs OHI (Calbacho 2004)

- $\mathrm{SRP}+\mathrm{OHI}+$ antimicrobial vs OHI (Engebretson 2013; Katagiri 2009; Raman 2014)

- $\mathrm{SRP}+\mathrm{OHI}+$ antimicrobial (doxycycline) + antimicrobial (chlorhexidine) vs usual treatment (Jones 2007)

- SRP + OHI + antimicrobial vs no active intervention (Singh 2008 (2 of 3 arms: Gp B vs Gp C); Sun 2011)

Comparison 2: Periodontal therapy vs alternative periodontal therapy $(n=26)$

\section{Subgroup 2.1: SRP vs alternative mechanical therapy $(n=1)$}

- $\mathrm{SRP}+\mathrm{OHI}$ vs mechanical therapy (supragingival cleaning) $+\mathrm{OHI}$ (Koromantzos 2011)

- $\mathrm{SRP}+\mathrm{OHI}+$ additional mechanical therapy (subgingival cleaning) vs SRP + $\mathrm{OHI}+$ additional mechanical therapy (supragingival cleaning) (Chen 2012 (2 of 3 arms: Gp A vs Gp B))

- Immediate SRP + OHI + additional mechanical therapy (prophylaxis) vs ongoing SRP + $\mathrm{OHI}+\mathrm{ad}-$ ditional mechanical therapy (prophylaxis) (Santos 2009)

- Immediate SRP + OHI + additional mechanical therapy (prophylaxis incl. subgingival debridement) vs ongoing SRP $+\mathrm{OHI}+$ additional mechanical therapy (prophylaxis incl. subgingival debridement) (Santos 2012)

Subgroup 2.3: SRP + antimicrobial vs antimicrobial $(n=1)$

- $\mathrm{SRP}+\mathrm{OHI}+$ antimicrobial (doxycycline) vs antimicrobial (doxycycline) (Yun 2007)

\section{Subgroup 2.4: SRP + antimicrobial vs SRP $(n=12)$}

- $\mathrm{SRP}+\mathrm{OHI}+$ antimicrobials vs SRP + OHI (Al-Zahrani 2009 (3 of 3 arms: Gps B (doxycycline) + C (antimicrobial photodynamic therapy (aPDT)) combined vs Gp A))

- $\mathrm{SRP}+\mathrm{OHI}+$ antimicrobial (subantimicrobial doxycycline) vs SRP + OHI (Gilowski 2012)

- $\mathrm{SRP}+\mathrm{OHI}+$ antimicrobial (chlorhexidine) vs SRP + OHI (Madden 2008)

- $\mathrm{SRP}+\mathrm{OHI}+$ antimicrobial (metronidazole) vs SRP + OHI (Miranda 2014)

- $\mathrm{SRP}+\mathrm{OHI}+$ antimicrobial (doxycycline) vs SRP + OHI (Singh 2008 (2 of 3 arms: Gp B vs Gp A); Tsalikis 2014)

- SRP + antimicrobials (doxycycline) vs SRP (Engebretson 2011 (3 of 3 arms: Gps A (subantimicrobial doxycycline) $+B$ (doxycycline) combined vs Gp C))

- SRP + antimicrobial (tetracycline) vs SRP (Haerian Ardakani 2014)

- SRP + antimicrobial (iodine) vs SRP (NCT00801164) 
Table 5. Periodontal therapies compared in included studies (Continued)

- $\mathrm{SRP}+\mathrm{OHI}+$ additional mechanical therapy (scale and polish) + antimicrobial (doxycycline) vs SRP $+\mathrm{OHI}+$ additional mechanical therapy (scale and polish) (O'Connell 2008)

- $\mathrm{SRP}+\mathrm{OHI}+$ additional mechanical therapy (prophylaxis) + antimicrobial (amoxicillin) vs SRP + $\mathrm{OHI}+$ additional mechanical therapy (prophylaxis) (Rodrigues 2003)

- $\mathrm{SRP}+\mathrm{OHI}+$ antimicrobial (chlorhexidine) + additional mechanical therapy (prophylaxis incl. subgingival debridement) vs SRP $+\mathrm{OHI}+$ additional mechanical therapy (prophylaxis incl. subgingival debridement) (Santos 2013)

- SRP + additional mechanical therapy (supragingival prophylaxis) + antimicrobial (minocycline) vs SRP + additional mechanical therapy (supragingival prophylaxis) (Skaleric 2004)

Subgroup 2.5: SRP + antimicrobial (doxycycline) vs SRP + alternative antimicrobial $(n=3)$

- $\mathrm{SRP}+\mathrm{OHI}+$ antimicrobial (doxycycline) vs SRP + OHI + alternative antimicrobial (aPDT) (Al-Zahrani 2009 (2 of 3 arms: Gp B vs Gp C))

- SRP + antimicrobial (doxycycline) vs SRP + alternative 'sub-' antimicrobial (doxycycline) (Engebretson 2011 (2 of 3 arms: Gp A vs Gp B))

- SRP + antimicrobial (doxycycline) vs SRP + alternative antimicrobial (chlorhexidine) (Grossi 1997 (2 of 5 arms: Gp A vs Gp D))

Subgroup 2.6: SRP + combined antimicrobials vs SRP + single antimicrobial $(n=4)$

- SRP + antimicrobial (chlorhexidine) + additional antimicrobial (doxycycline) vs SRP + antimicrobial (chlorhexidine) (Grossi 1997 (2 of 5 arms: Gp B vs Gp D))

- SRP + antimicrobial (doxycycline) + additional antimicrobial (iodine) vs SRP + antimicrobial (doxycycline) (Grossi 1997 (2 of 5 arms: Gp C vs Gp A))

- $\mathrm{SRP}+\mathrm{OHI}+$ antimicrobial (1: chlorhexidine rinse) + antimicrobial (2: systemic doxycycline) vs SRP $+\mathrm{OHI}+$ antimicrobial (1: chlorhexidine rinse) (Llambés 2008)

- $\mathrm{SRP}+\mathrm{OHI}+$ antimicrobial (1: doxycycline) + antimicrobial (2: aPDT) vs SRP + OHI + antimicrobial (1: doxycycline) (Macedo 2014)

N.B. In analysis, Grossi 1997 estimate is presented combined effect from Gps B + C vs Gps A + D to use maximum data

Subgroup 2.7: SRP + bone modifier vs SRP $(n=1)$

- $\mathrm{SRP}+\mathrm{OHI}+$ bone modifier (aminobisphosphanate) vs SRP + OHI (Rocha 2001)

Subgroup 2.8: SRP + statin vs SRP $(n=1)$

- $\mathrm{SRP}+\mathrm{OHI}+$ statin (simvastatin) vs SRP + OHI (Pradeep 2013)

aPDT = antimicrobial photodynamic therapy; $\mathrm{OHI}=$ oral hygiene instruction; $\mathrm{SRP}=$ scaling and root planing; vs = versus

Table 6. Secondary outcomes: Comparison 1. Periodontal therapy versus no active intervention/usual care

\begin{tabular}{|c|c|c|c|c|c|}
\hline Outcome & Time point & $\begin{array}{l}\text { Number of } \\
\text { studies }\end{array}$ & $\begin{array}{l}\text { Control } \\
\text { group } \\
\text { weighted } \\
\text { mean }\end{array}$ & Mean difference (IV, 95\% Cl; P value) & $\begin{array}{l}\text { Heterogeneity (P } \\
\text { value; } I^{2} \text { ) }\end{array}$ \\
\hline \multirow[t]{2}{*}{ BOP } & 3-4 months & 4 & 0.059 & $\begin{array}{l}-0.16,95 \% \mathrm{Cl}-0.21 \text { to }-0.10 \text { (Random); } \mathrm{P} \\
<0.00001\end{array}$ & $(P=0.06) ; I^{2}=59 \%$ \\
\hline & 6 months & 3 & 0.004 & $\begin{array}{l}-0.14,95 \% \mathrm{Cl}-0.17 \text { to }-0.10 \text { (Random); } \mathrm{P} \\
<0.00001\end{array}$ & $(P=0.26) ; I^{2}=25 \%$ \\
\hline
\end{tabular}


Table 6. Secondary outcomes: Comparison 1. Periodontal therapy versus no active intervention/usual care (Continued)

\begin{tabular}{|c|c|c|c|c|c|}
\hline \multirow[t]{2}{*}{ CAL } & 3-4 months & 8 & 1.534 & $\begin{array}{l}-0.25,95 \% \mathrm{Cl}-0.45 \text { to }-0.05 \text { (Random); P } \\
=0.01\end{array}$ & $\begin{array}{l}(P=0.0005) ; 1^{2}= \\
73 \%\end{array}$ \\
\hline & 6 months & 5 & 1.308 & $\begin{array}{l}-0.41,95 \% \mathrm{Cl}-0.71 \text { to }-0.11 \text { (Random); P } \\
=0.008\end{array}$ & $\begin{array}{l}(P<0.0001) ; 1^{2}= \\
85 \%\end{array}$ \\
\hline \multirow[t]{2}{*}{ GI } & 3-4 months & 4 & 0.182 & $\begin{array}{l}-0.54,95 \% \mathrm{Cl}-0.80 \text { to }-0.27 \text { (Random); } \mathrm{P} \\
<0.0001\end{array}$ & $\begin{array}{l}(P<0.0003) ; 1^{2}= \\
84 \%\end{array}$ \\
\hline & 6 months & 1 & 1.3 & $\begin{array}{l}-0.30,95 \% \mathrm{Cl}-0.41 \text { to }-0.19 \text { (Fixed); } \mathrm{P}< \\
0.00001\end{array}$ & $\mathrm{n} / \mathrm{a}$ \\
\hline \multirow[t]{2}{*}{ PI } & 3-4 months & 7 & 1.566 & $\begin{array}{l}-0.57,95 \% \mathrm{Cl}-0.79 \text { to }-0.35 \text { (Random); } \mathrm{P} \\
<0.00001\end{array}$ & $\begin{array}{l}(P<0.00001) ; I^{2}= \\
88 \%\end{array}$ \\
\hline & 6 months & 2 & 0.390 & $\begin{array}{l}-0.41(-0.51 \text { to }-0.30)(\text { Fixed); } \mathrm{P}< \\
0.00001\end{array}$ & $(P=0.09) ; I^{2}=66 \%$ \\
\hline \multirow[t]{2}{*}{ PPD } & 3-4 months & 9 & 1.303 & $\begin{array}{l}-0.40,95 \% \mathrm{Cl}-0.62 \text { to }-0.18 \text { (Random); } \mathrm{P} \\
<0.0004\end{array}$ & $\begin{array}{l}(P<0.00001) ; I^{2}= \\
88 \%\end{array}$ \\
\hline & 6 months & 4 & 0.364 & $\begin{array}{l}-0.34,95 \% \mathrm{Cl}-0.44 \text { to }-0.25 \text { (Random); } \mathrm{P} \\
<0.00001\end{array}$ & $(P=0.23) ; I^{2}=30 \%$ \\
\hline
\end{tabular}

$\mathrm{BOP}=$ bleeding on probing; $\mathrm{CAL}=$ clinical attachment level; $\mathrm{Cl}=$ confidence interval; $\mathrm{GI}$ = gingival index; $\mathrm{PI}=$ plaque index; $\mathrm{PPD}=$ probing pocket depth 


\begin{tabular}{|c|c|c|c|c|c|c|c|}
\hline Outcome & Time point & Subgroup & $\begin{array}{l}\text { Pooled num- } \\
\text { ber of studies }\end{array}$ & $\begin{array}{l}\text { Single studies } \\
\text { (unsuitable for } \\
\text { pooling) }\end{array}$ & $\begin{array}{l}\text { Control } \\
\text { group mean } \\
\text { (weighted } \\
\text { mean where } \\
\text { pooled) }\end{array}$ & $\begin{array}{l}\text { Mean difference (IV, } 95 \% \mathrm{Cl} \text {; P } \\
\text { value) }\end{array}$ & $\begin{array}{l}\text { Heterogene- } \\
\text { ity (P value; } \\
I^{2} \text { ) }\end{array}$ \\
\hline \multirow[t]{12}{*}{ BOP } & 3-4 months & $\begin{array}{l}\text { SRP vs alternative mechani- } \\
\text { cal therapy }\end{array}$ & $\mathrm{n} / \mathrm{a}$ & $\begin{array}{l}\text { Koromantzos } \\
20111\end{array}$ & 0.597 & $-0.22(-0.34,-0.11) ; P=0.0001$ & $\mathrm{n} / \mathrm{a}$ \\
\hline & & SRP vs alternative SRP & $\mathrm{n} / \mathrm{a}$ & Chen $2012^{2}$ & 0.121 & $0.01(-0.03,0.05) ; P=0.53$ & $\mathrm{n} / \mathrm{a}$ \\
\hline & & & & Santos 20093 & 0.119 & $-0.03(-0.09,0.03) ; P=0.33$ & \\
\hline & & & & Santos 20124 & 0.109 & $-0.02(-0.09,0.04) ; P=0.44$ & \\
\hline & & SRP + antimicrobial vs SRP & 6 & $\mathrm{n} / \mathrm{a}$ & 0.172 & $\begin{array}{l}0.01(-0.03,0.05)(\text { Random); } P= \\
0.52\end{array}$ & $\begin{array}{l}(P=0.23) ; 1^{2}= \\
27 \%\end{array}$ \\
\hline & & $\begin{array}{l}\text { SRP }+ \text { antimicrobial vs an- } \\
\text { timicrobial }\end{array}$ & $\mathrm{n} / \mathrm{a}$ & Yun 2007 & 0.582 & $-0.16(-0.35,0.03) ; P=0.10$ & $\mathrm{n} / \mathrm{a}$ \\
\hline & & $\begin{array}{l}\text { SRP + combined antimicro- } \\
\text { bials vs SRP + single antimi- } \\
\text { crobial }\end{array}$ & $\mathrm{n} / \mathrm{a}$ & Macedo 2014 & 0.157 & $-0.01(-0.08,0.06) ; P=0.78$ & $\mathrm{n} / \mathrm{a}$ \\
\hline & 6 months & $\begin{array}{l}\text { SRP vs alternative mechani- } \\
\text { cal therapy }\end{array}$ & $\mathrm{n} / \mathrm{a}$ & $\begin{array}{l}\text { Koromantzos } \\
2011^{1}\end{array}$ & 0.617 & $-0.24(-0.35,-0.13) ; P<0.0001$ & $\mathrm{n} / \mathrm{a}$ \\
\hline & & SRP vs alternative SRP & $\mathrm{n} / \mathrm{a}$ & Chen $2012^{2}$ & 0.12 & $0.02(-0.25,0.29) ; P=0.89$ & $\mathrm{n} / \mathrm{a}$ \\
\hline & & & & Santos 20093 & 0.114 & $-0.03(-0.11,0.04) ; P=0.38$ & \\
\hline & & & & Santos 20124 & 0.102 & $-0.03(-0.11,0.04) ; P=0.37$ & \\
\hline & & SRP + antimicrobial vs SRP & 2 & $\mathrm{n} / \mathrm{a}$ & 0.116 & $0.02(-0.03,0.07)($ Fixed); $P=0.46$ & $\begin{array}{l}(P=0.45) ; 1^{2}= \\
0 \%\end{array}$ \\
\hline \multirow[t]{3}{*}{ CAL } & 3-4 months & SRP vs alternative SRP & $\mathrm{n} / \mathrm{a}$ & Chen $2012^{2}$ & 2.55 & $0.73(0.22,1.24) ; P=0.005$ & $\mathrm{n} / \mathrm{a}$ \\
\hline & & & & Santos 20093 & 3.5 & $-0.30(-0.76,0.16) ; P=0.20$ & \\
\hline & & & & Santos 20124 & 3.5 & $-0.40(-0.87,0.07) ; P=0.10$ & \\
\hline
\end{tabular}




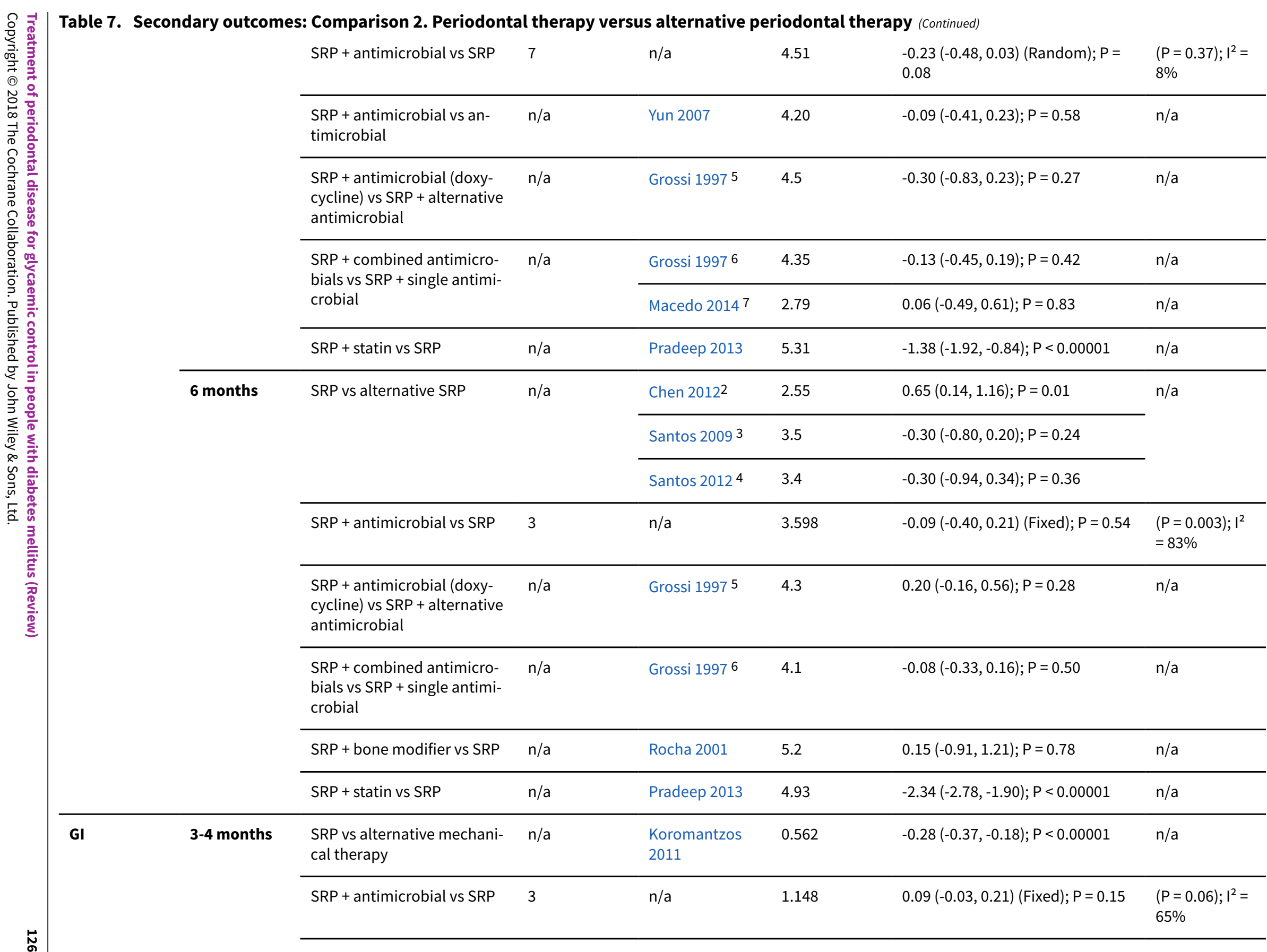




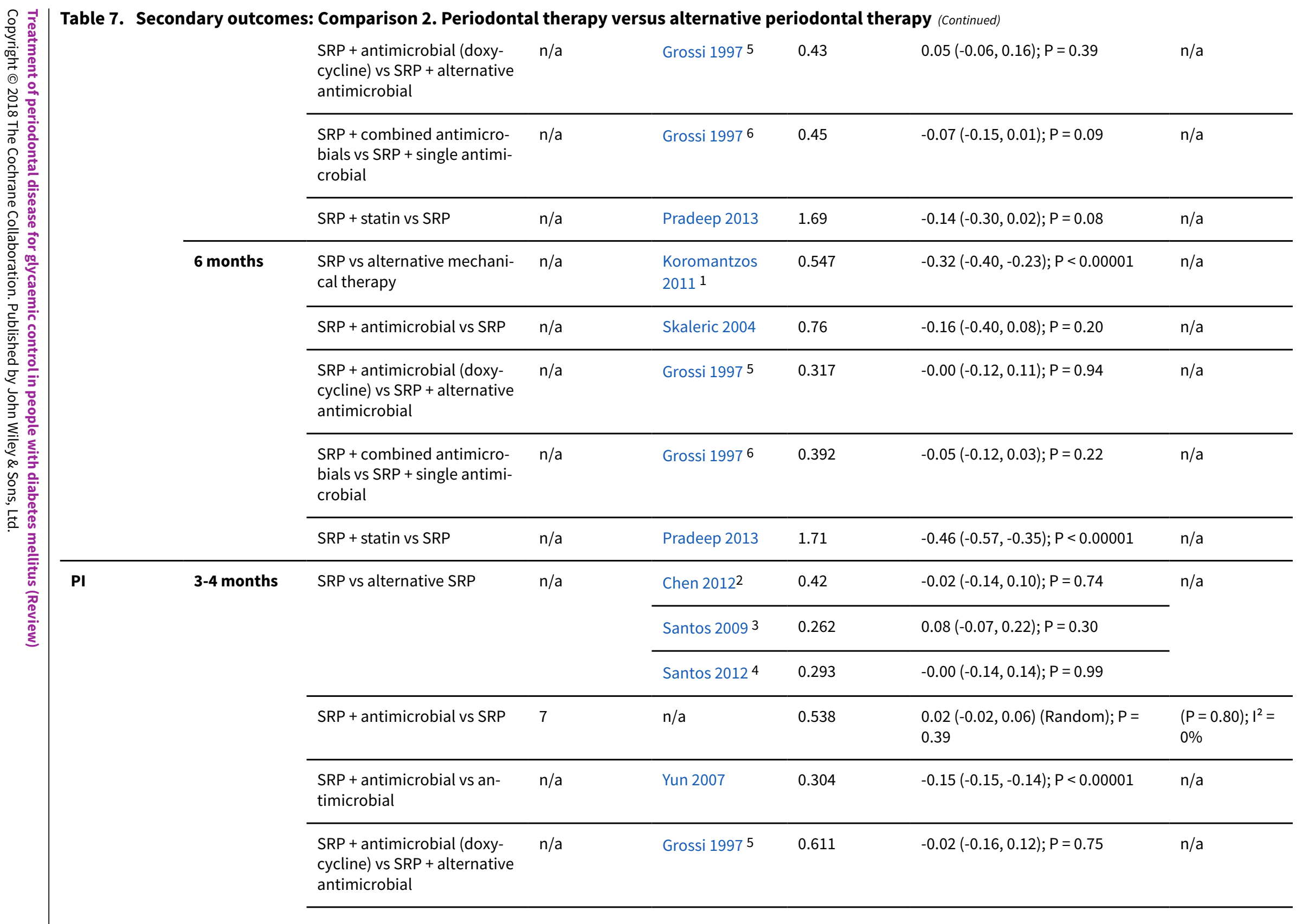




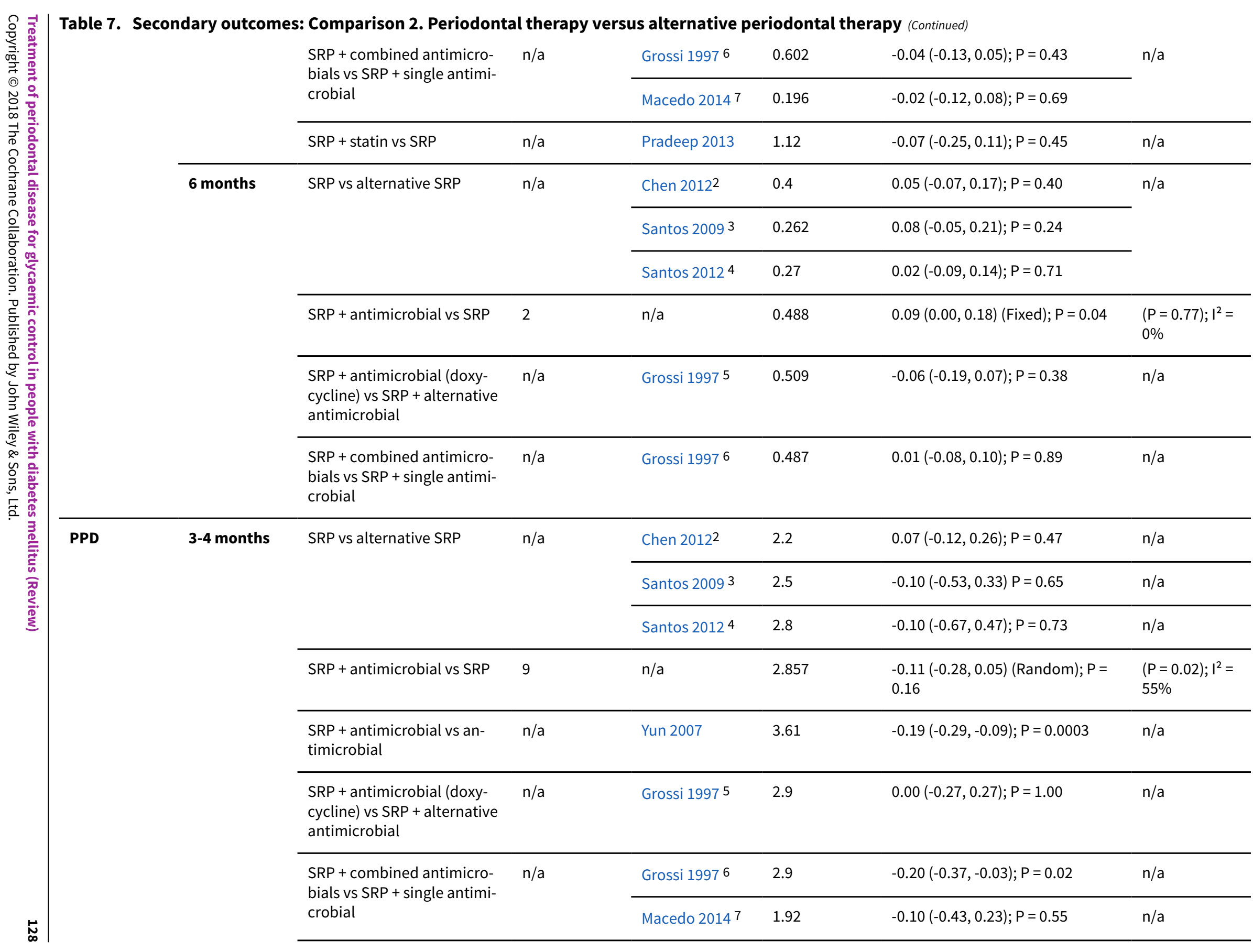




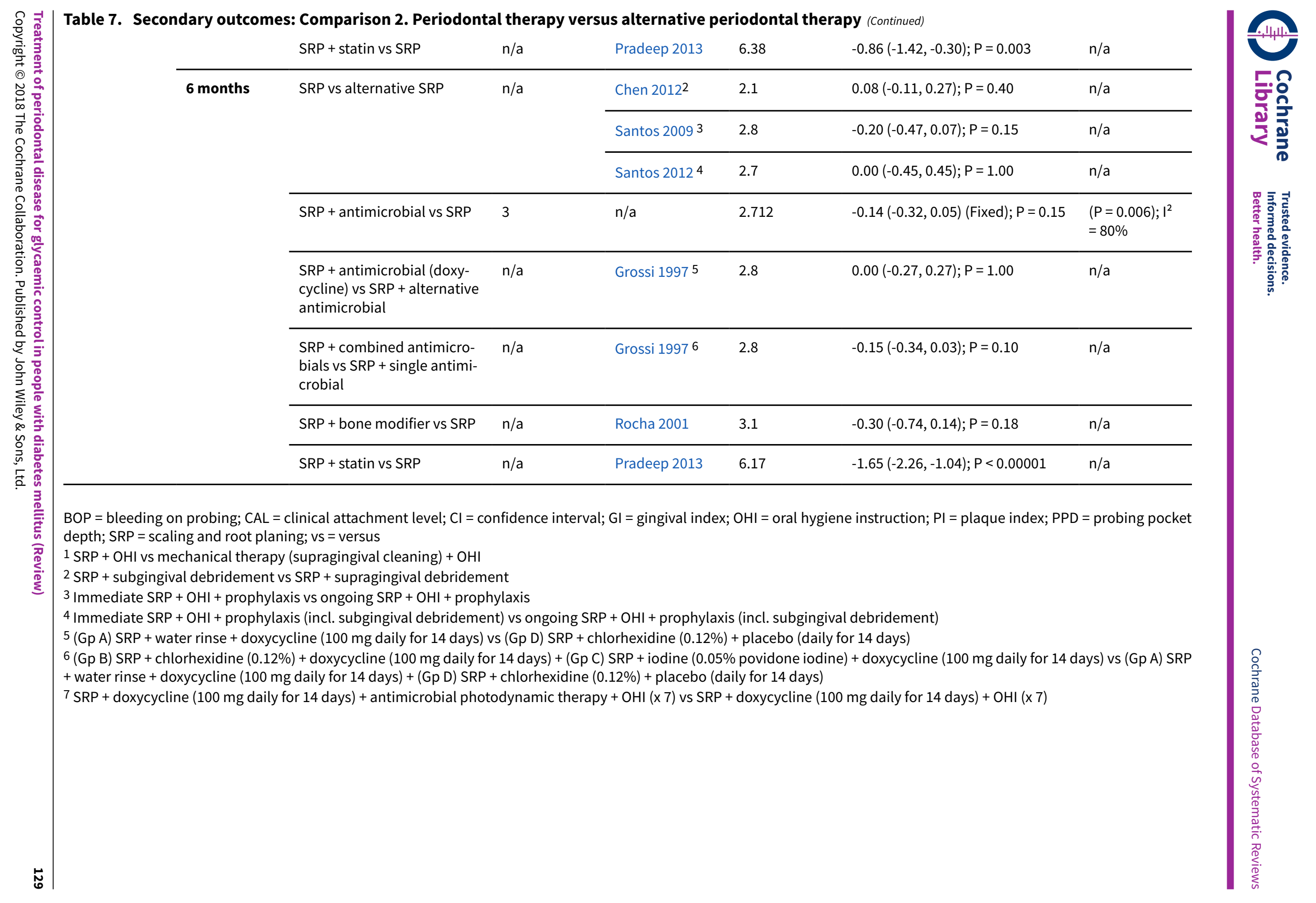




\title{
AP PEN DICES
}

\section{Appendix 1. Search strategies}

\section{The Cochrane Oral Health Group Trials Register search strategy}

From April 2013, searches of the Cochrane Oral Health Group Trials Register were carried out in the Cochrane Register of Studies using the search strategy below:

\author{
\#1 (diabet* or IDDM OR DMI OR MODY OR DM2 OR NIDDM OR IIDM):ti,ab \\ \#2 periodont*:ti,ab \\ \#3 (\#1 and \#2) AND (INREGISTER)
}

Previous searches of the Cochrane Oral Health Group Trials Register were carried out using the Procite software and the search strategy below:

((diabet* or IDDM OR DMI OR MODY OR DM2 OR NIDDM OR IIDM)and periodont*)

\section{The Cochrane Central Register of Controlled Trials (CENTRAL) search strategy}

\#1 MeSH descriptor DIABETES MELLITUS explode all trees

\#2 (diabet* in Abstract or diabet* in Record Title)

\#3 (dka in All Text or iddm in All Text)

\#4 (dmi in Record Title or dmi in Abstract)

\#5 (mody in All Text or dm2 in All Text or niddm in All Text)

\#6 (iidm in Record Title or iidm in Abstract)

\#7 insulin* next secret* next dysfunc* in All Text

\#8 (insulin* next resist* in Record Title or insulin* next resist* in Abstract)

\#9 ((impaired next glucose next tolerance in All Text or glucose next intoleran* in All Text or insulin* next resist* in Record Title) and (DM in Record Title or DM in Abstract or DM2 in Record Title or DM2 in Abstract))

\#10 ((juvenile* in All Text or child* in All Text or keto* in All Text or labil* in All Text or brittl ${ }^{\star}$ in All Text or "early onset" in All Text) and (diabetes in All Text or DM in All Text or DM1 in All Text))

\#11 (("keto* prone" in All Text near/6 diabet* in All Text) or (autoimmun* in All Text near/6 diabet* in All Text) or ("auto immun" in All Text near/6 diabet* in All Text) or ("sudden onset" in All Text near/6 diabet* in All Text))

\#12 ((keto* in All Text and (resist* in All Text near/6 diabet* in All Text)) or (nonketo* in All Text near/6 diabet* in All Text) or (non in All Text and (keto* in All Text near/6 diabet* in All Text)) or (adult* in All Text and (onset in All Text near/6 diabet ${ }^{\star}$ in All Text)) or (matur ${ }^{\star}$ in All Text and (onset in All Text near/6 diabet* in All Text)) or (late* in All Text and (onset in All Text near/6 diabet* in All Text)) or (slow* in All Text and (onset in All Text near/6 diabet* in All Text)) or (stabl* in All Text near/6 diabet* in All Text))

\#13 MeSH descriptor INSULIN RESISTANCE explode all trees

\#14 ("insulin* depend*" in All Text or "noninsulin* depend*" in All Text or "non insulin-depend*" in All Text or (typ* in All Text and (I in All Text near/6 diabet* in All Text)) or (typ* in All Text and (II in All Text near/6 diabet* in All Text)))

\#15 ((insulin* in All Text and (defic* in All Text near/6 absolut in All Text)) or (insulin* in All Text and (defic* in All Text near/6 relativ* in All Text)))

\#16 ((metabolic* in All Text and syndrom* in Record Title) or (metabolic* in All Text and syndrom* in Abstract) or (plurimetabolic ${ }^{*}$ in All Text and syndrom* in Record Title) or (plurimetabolic* in All Text and syndrom* in Abstract) or (pluri in All Text and metabolic* in All Text and syndrom* in Record Title) or (pluri in All Text and metabolic* in All Text and syndrom* in Abstract))

$\# 17$ (\#1 or \#2 or \#3 or \#4 or \#5 or \#6 or \#7 or \#8 or \#9 or \#10 or \#11 or \#12 or \#13 or \#14 or \#15 or \#16)

\#18 MeSH descriptor PERIODONTICS explode all trees

\#19 MeSH descriptor PERIODONTAL DISEASES explode all trees

\#20 MeSH descriptor PREVENTIVE DENTISTRY explode all trees

\#21 MeSH descriptor Dental Care for Chronically Ill explode all trees

\#22 (periodont* in All Text or gingivitis in All Text or gingiva* in All Text)

\#23 MeSH descriptor DENTAL PROPHYLAXIS explode all trees

\#24 ((scale* in All Text near/6 polish* in All Text) or (scaling in All Text near/6 polish* in All Text) or (root in All Text near/6 plane in All Text) or (root in All Text near/6 planed in All Text) or (root in All Text near/6 planing in All Text))

\#25 MeSH descriptor SURGICAL FLAPS explode all trees

\#26 ((\#25 or (surgical in All Text and flap* in All Text) ) and periodont* in All Text)

\#27 ((tooth in All Text near/6 scaling in All Text) or (teeth in All Text near/6 scaling in All Text) or (dental in All Text near/6 scaling in All Text))

\#28 ((tooth in All Text near/6 scale* in All Text) or (teeth in All Text near/6 scale* in All Text) or (dental in All Text near/6 scale* in All Text))

\#29 ((oral in All Text near/6 prophylaxis in All Text) or (dental in All Text near/6 prophylaxis in All Text))

\#30 MeSH descriptor ORAL HYGIENE this term only

\#31 MeSH descriptor ORAL HEALTH this term only

\#32 (oral next hygien* in All Text or oral next health* in All Text) 
$\# 33$ (\#18 or \#19 or \#20 or \#21 or \#22 or \#23 or \#24 or \#25 or \#26 or \#27 or \#28 or \#29 or \#30 or \#31 or \#32)

\#34 (\#17 and \#33)

\section{MEDLINE via OVID search strategy}

1. exp Diabetes Mellitus/

2. diabet\$.ab,ti.

3. (DKA or IDDM).mp. or DMI.ab,ti. [mp=title, original title, abstract, name of substance word, subject heading word]

4. (MODY or DM2 or NIDDM).mp. or IIDM.ti,ab. [mp=title, original title, abstract, name of substance word, subject heading word]

5. insulin\$ secret\$ dysfunc\$.ti,ab.

6. insulin\$ resist\$.ti,ab.

7. ((impaired glucose tolerance or glucose intoleran $\$$ or insulin\$ resist\$) and (DM or DM2)).ti,ab.

8. insulin\$ depend\$.mp. or insulin?depend\$.ti,ab. [mp=title, original title, abstract, name of substance word, subject heading word]

9. (non insulin\$ depend\$ or nonisulin\$ depend\$ or nonisulin?depend).mp. or non insulin?depend\$.ti,ab. [mp=title, original title, abstract, name of substance word, subject heading word]

10.(("typ\$1" or typ\$I) adj6 DM).ti,ab.

11.(("typ\$2" or typ\$ II) adj6 DM).ti,ab.

12.((juvenil\$ or child\$ or keto\$ or labil\$ or brittl\$ or earl\$ onset) adj6 (DM or DM1)).ti,ab.

13.((keto\$ prone or autoimmun\$ or auto immun\$ or sudden onset) adj6 (DM or DM1)).ti,ab.

14.((keto\$ resist\$ or nonketo\$ or non keto $\$$ or adult\$ onset or matur\$ onset or late\$ onset or slow onset or stabl\$) adj6 (DM or DM2)).ti,ab.

15.exp Insulin Resistance/

16.(insulin\$ defic\$ adj6 (absolut\$ or relativ\$)).ti,ab.

17. metabolic $\$$ syndrom $\$ . t i, a b$.

18.(syndrom $\$$ X not (fragil\$ X or X linked)).ti,ab.

19. (plurimetabolic $\$$ syndrom $\$$ or pluri metabolic $\$$ syndrom $\$$ ).ti,ab.

20.or/1-19

21.exp Periodontics/

22.exp Periodontal Diseases/

23.exp Preventive Dentistry/

24.exp Dental Care for Chronically IIl/

25.periodont\$.mp. [mp=title, original title, abstract, name of substance word, subject heading word]

26.Surgical Flaps/

27.surgical flap\$.mp. [mp=title, original title, abstract, name of substance word, subject heading word]

28.(26 or 27) and periodont\$.mp. [mp=title, original title, abstract, name of substance word, subject heading word]

29.exp Dental Prophylaxis/

30.(scale\$ adj4 polish\$).mp. [mp=title, original title, abstract, name of substance word, subject heading word]

31.(scaling adj4 polish\$).mp. [mp=title, original title, abstract, name of substance word, subject heading word]

32.((root\$ adj4 planing) or (root\$ adj4 plan\$)).mp. [mp=title, original title, abstract, name of substance word, subject heading word]

33. (gingivitis or gingiva\$).mp. [mp=title, original title, abstract, name of substance word, subject heading word]

34.((tooth adj6 scaling) or (teeth adj6 scaling) or (dental adj6 scaling)).mp. [mp=title, original title, abstract, name of substance word, subject heading word]

35.(((tooth adj6 scale\$) or teeth) adj6 scale\$).mp. [mp=title, original title, abstract, name of substance word, subject heading word]

36.(((oral adj3 prophylaxis) or dental) adj3 prophylaxis).mp. [mp=title, original title, abstract, name of substance word, subject heading word]

37. Oral Hygiene/

38. Oral Health/

39. (oral hygien\$ or oral health\$).mp. [mp=title, original title, abstract, name of substance word, subject heading word]

40.or/21-25

41.or/28-40

42.or/40-41

43.20 and 42

The above subject search was linked to the Cochrane Highly Sensitive Search Strategy (CHSSS) for identifying randomised trials in MEDLINE: sensitivity maximising version (2008 revision) as referenced in Chapter 6.4.11.1 and detailed in box 6.4.c of theCochrane Handbook for Systematic Reviews of Interventions version 5.1.0 (updated March 2011). 
1. randomized controlled trial.pt.

2. controlled clinical trial.pt.

3. randomized.ab.

4. placebo.ab.

5. drug therapy.fs.

6. randomly.ab.

7. trial.ab.

8. groups.ab.

9. or/1-8

10. exp animals/ not humans.sh.

11.9 not 10

\section{EMBASE via OVID search strategy}

1. exp Diabetes Mellitus/

2. diabet\$.ab,ti.

3. (DKA or IDDM).mp. or DMI.ab,ti. [mp=title, abstract, subject headings, drug trade name, original title, device manufacturer, drug manufacturer name]

4. (MODY or DM2 or NIDDM).mp. or IIDM.ti,ab. [mp=title, abstract, subject headings, drug trade name, original title, device manufacturer, drug manufacturer name]

5. insulin\$ secret\$ dysfunc\$.ti,ab.

6. insulin\$ resist\$.ti,ab.

7. ((impaired glucose tolerance or glucose intoleran\$ or insulin\$ resist\$) and (DM or DM2)).ti,ab.

8. insulin\$ depend\$.mp. or insulin?depend\$.ti,ab.

9. (non insulin\$ depend\$ or nonisulin $\$$ depend\$ or nonisulin?depend).mp. or non insulin?depend\$.ti,ab.

10. (("typ\$1" or typ\$I) adj6 DM).ti,ab.

11. (("typ\$2" or typ\$ II) adj6 DM).ti,ab.

12. ((juvenil\$ or child\$ or keto\$ or labil\$ or brittl\$ or earl\$ onset) adj6 (DM or DM1)).ti,ab.

13. ((keto\$ prone or autoimmun\$ or auto immun\$ or sudden onset) adj6 (DM or DM1)).ti,ab.

14. ((keto $\$$ resist $\$$ or nonketo $\$$ or non keto $\$$ or adult $\$$ onset or matur $\$$ onset or late $\$$ onset or slow onset or stabl\$) adj6 (DM or DM2)).ti,ab.

15. exp Insulin Resistance/

16. (insulin\$ defic\$ adj6 (absolut\$ or relativ\$)).ti,ab.

17. metabolic\$ syndrom\$.ti,ab.

18. (syndrom $\$ X$ not (fragil\$ $X$ or $X$ linked)).ti,ab.

19. (plurimetabolic $\$$ syndrom $\$$ or pluri metabolic $\$$ syndrom\$).ti,ab.

20. or/1-19

21. exp Periodontics/

22. exp Periodontal Disease/

23. exp Preventive Dentistry/

24. Dental Care.mp. and Chronic\$ill\$

25. periodont\$.mp. [mp=title, abstract, subject headings, drug trade name, original title, device manufacturer, drug manufacturer name]

26. (surgical flap\$ and periodont\$).mp.

27. exp Dental Prophylaxis/

28. (scale\$ adj4 polish\$).mp.

29. (scaling adj4 polish\$).mp.

30. ((root\$ adj4 planing) or (root\$ adj4 plan\$)).mp.

31. (gingivitis or gingiva\$).mp.

32. ((tooth adj6 scaling) or (teeth adj6 scaling) or (dental adj6 scaling)).mp.

33. (((tooth adj6 scale\$) or teeth) adj6 scale\$).mp.

34. (((oral adj3 prophylaxis) or dental) adj3 prophylaxis).mp.

35. Mouth Hygiene/

36. (oral hygien or oral health\$).mp.

37. or/21-36

38. 20 and 37

The above subject search was linked to the Cochrane Oral Health Group filter for identifying randomised controlled trials in EMBASE via OVID:

1. random\$.ti,ab.

2. factorial\$.ti,ab.

3. (crossover\$ or cross over\$ or cross-over\$).ti,ab.

4. placebo\$.ti,ab.

5. (doubl\$ adj blind\$).ti,ab.

Treatment of periodontal disease for glycaemic control in people with diabetes mellitus (Review) 
6. (singl\$ adj blind\$).ti,ab.

7. assign $\$ . t i, a b$.

8. allocat\$.ti,ab.

9. volunteer\$.ti,ab.

10. CROSSOVER PROCEDURE.sh.

11. DOUBLE-BLIND PROCEDURE.sh.

12. RANDOMIZED CONTROLLED TRIAL.sh.

13. SINGLE BLIND PROCEDURE.sh.

14. or/1-13

15. (exp animal/ or animal.hw. or nonhuman/) not (exp human/ or human cell/ or (human or humans).ti.)

16. 14 NOT 15

\section{CINAHL via EBSCO search strategy}

S1 MH "DIABETES MELLITUS+"

S2 TI diabet*

S3 AB diabet*

S4 DKA or IDDM or TI DMI or AB DMI

S5 MODY or DM2 or NIDDM or TI IDDM or AB IDDM

S6 TI insulin ${ }^{\star}$ secret $^{\star}$ dysfunc ${ }^{\star}$ or AB insulin ${ }^{\star}$ secret $^{\star}$ dysfunc $^{\star}$

S7 TI insulin* resist ${ }^{\star}$ or $A B$ insulin* resist*

S8 impaired glucose tolerance or glucose intoleran* or insulin* resist*

S9 TI DM or AB DM or TI DM2 or AB DM2

S10 S9 and S8

S11 insulin* depend* or AB insulin* depend ${ }^{\star}$ or TI insulin* depend*

S12 non insulin ${ }^{\star}$ depend $^{\star}$ or nonisulin ${ }^{\star}$ depend $^{\star}$ or non isulin* depend $^{\star}$

S13 "typ* 1 " or "typ* I"

S14 TI DM or AB DM

S15 S14 and S13

S16 "typ* 2 " or "typ* II"

S17 S16 and S14

S18 TI DM or AB DM or TI DM1 or AB DM1

S19 juvenil* or child* or keto* or labil* or brittl* or "earl* onset"

S20 S19 and S18

S21 keto* prone or autoimmun* or auto immun* or "sudden onset"

S22 S21 and S18

S23 keto resist* or nonketo* or non keto* or "adult* onset" or matur* or "late* onset" or "slow onset" or stabl*

S24 S23 and S18

S25 MH INSULIN RESISTANCE

S26 insulin* defic*

S27 TI metabolic* syndrom* or AB metabolic* syndrom*

S28 syndrom* $X$ not ( fragil ${ }^{\star} X$ or $X$ linked)

S29 TI plurimetabolic syndrom $^{\star}$ or AB plurimetabolic* syndrom* or TI pluri metabolic syndrom $^{\star}$ or AB pluri metabolic syndrom $^{\star}$

$\mathrm{S} 30 \mathrm{~S} 29$ or S28 or S27 or S26 or S25 or S24 or S22 or S20 or S17 or S15 or S12 or S11 or S10 or S7 or S6 or S5 or S4 or S3 or S2 or S1

S31 MH PERIODONTICS or MH PERIODONTAL DISEASES or MH PREVENTIVE DENTISTRY or MH DENTAL CARE FOR CHRONICALLY ILL

S32 periodont*

S33 MH SURGICAL FLAPS or surgical flap*

S34 S33 and S32

S35 MH DENTAL PROPHYLAXIS

S36 scale or scaling and polish

S37 root and plan*

S38 gingivitis or gingiva*

S39 (tooth or teeth or dental) and scal*

S40 (oral or dental) and prophylaxis

S41 MH ORAL HYGIENE or oral hygien* or oral health*

S42 S41 or S40 or S39 or S38 or S37 or S36 or S35 or S34 or S32 or S31

S43 S42 and S30

The above subject search was linked to the Cochrane Oral Health Group filter for identifying controlled trials in CINAHL:

S1 MH Random Assignment

S2 MH Single-blind studies

S3 MH double-blind studies

Treatment of periodontal disease for glycaemic control in people with diabetes mellitus (Review) 
S4 MH triple-blind studies

S5 MH crossover design

$\mathrm{S} 6 \mathrm{MH}$ factorial design

S7 multicentre study or multicenter study or multi-centre study or multi-center study

$\mathrm{S} 8 \mathrm{TI}$ random or $\mathrm{AB}$ random

S9 $\mathrm{TI}$ latin square or $\mathrm{AB}$ latin square

$\mathrm{S} 10 \mathrm{TI}$ crossover or AB crossover or TI cross-over or AB cross-over

S11 MH placebos

S12 (singl ${ }^{\star}$ or doubl ${ }^{\star}$ or trebl ${ }^{\star}$ or tripl $\left.l^{\star}\right)$ and $\left(\right.$ blind $^{\star}$ or mask $\left.^{\star}\right)$

S13 MH clinical trials

S14 placebo*

S15 clinical and trial

$\mathrm{S} 16 \mathrm{~S} 1$ or $\mathrm{S} 2$ or $\mathrm{S} 3$ or $\mathrm{S} 4$ or $\mathrm{S} 5$ or $\mathrm{S} 6$ or $\mathrm{S} 7$ or $\mathrm{S} 8$ or $\mathrm{S} 9$ or $\mathrm{S} 10$ or $\mathrm{S} 11$ or $\mathrm{S} 12$ or $\mathrm{S} 13$ or $\mathrm{S} 14$ or $\mathrm{S} 15$

\section{LILACS via BIREME Virtual Health Library search strategy}

diabet\$ [Palavras]

and periodont\$ [Palavras]

The above subject search was linked to the Brazilian Cochrane Centre filter for identifying randomised controlled trials in LILACS:

((Pt RANDOMIZED CONTROLLED TRIAL OR Pt CONTROLLED CLINICAL TRIAL OR Mh RANDOMIZED CONTROLLED TRIALS OR Mh RANDOM ALLOCATION OR Mh DOUBLE-BLIND METHOD OR Mh SINGLE-BLIND METHOD OR Pt MULTICENTER STUDY) OR ((tw ensaio or tw ensayo or tw trial) and (tw azar or tw acaso or tw placebo or tw control\$ or tw aleat\$ or tw random\$ or (tw duplo and tw cego) or (tw doble and tw ciego) or (tw double and tw blind)) and tw clinic\$)) AND NOT ((CT ANIMALS OR MH ANIMALS OR CT RABBITS OR CT MICE OR MH RATS OR MH PRIMATES OR MH DOGS OR MH RABBITS OR MH SWINE) AND NOT (CT HUMAN AND CT ANIMALS)) [Palavras]

\section{ZETOC Conference Proceedings search strategy}

diabet* AND periodont* $^{\star}$

ISI Web of Knowledge Conference Proceedings search strategy

diabet* AND periodont* $^{\star}$

US National Institutes of Health Trials Registry (ClinicalTrials.gov) and WHO International Clinical Trials Registry Platform search strategy

periodontal AND diabetes

WHAT'S NEW

\begin{tabular}{lll}
\hline Date & Event & Description \\
\hline 13 March 2018 & Amended & $\begin{array}{l}\text { Minor typographical error corrected in references (Firatli). Minor } \\
\text { numerical error corrected in Characteristics of included studies } \\
\text { table (Santos 2009). }\end{array}$ \\
\hline
\end{tabular}

\section{H I S T O R Y}

Protocol first published: Issue 2, 2004

Review first published: Issue 5, 2010

\begin{tabular}{lll}
\hline Date & Event & Description \\
\hline 5 November 2015 & New search has been performed & Search run up to December 2014 \\
\hline 5 November 2015 & $\begin{array}{l}\text { New citation required but conclusions } \\
\text { have not changed }\end{array}$ & $\begin{array}{l}\text { Review now contains 35 included studies. The previous version } \\
(2010) \text { had 7 included studies. New authors involved }\end{array}$ \\
\hline \hline
\end{tabular}

Treatment of periodontal disease for glycaemic control in people with diabetes mellitus (Review) 


\section{CONTRIBUTIONS OF AUTHORS}

Conception/design of the review: Terry C Simpson (TS).

Co-ordination of the review: Jo C Weldon (JW).

Writing the protocol: TS, Ian Needleman (IN), Sarah H Wild (SW), David R Moles (DM), Susan Furness (SF), Edward Mills (EM).

Developing search strategy: Sylvia Bickley (Cochrane OHG), TS.

Running electronic searches: Anne Littlewood (Cochrane OHG).

Communication with authors and organisations:TS, JW, SF, Helen V Worthington (HW).

Screening titles, abstracts and full text papers: TS, SF, HW, JW, Zipporah Iheozor-Ejiofor (ZIE).

Arbiter for inclusion/exclusion of papers: ZIE.

Extracting data: TS, SF, HW, JW, ZIE, IN, DM, Brian Stevenson (BS).

Appraising quality/risk of bias: ZIE, JW.

Inputting numerical data: JW, HW.

Analysis of data: HW, JW.

Risk of bias analysis: ZIE, JW.

Interpretation of data: HW, JW.

Summary of findings tables: ZIE, JW.

Writing the review: TS, JW, ZIE, SF, HW.

Reviewing draft review/providing comments: IN, BS, DM, SF, SW, TS, HW, JW, ZIE.

\section{5 update}

Edward Mills was not able to be involved in this update. Susan Furness, Zipporah Iheozor-Ejiofor, Brian Stevenson and Jo Weldon were added to the review team.

\section{DECLARATIONS OF INTEREST}

Terry C Simpson (TS): none known.

Jo Weldon (JW): none known. JW is salaried member of staff of the Cochrane Oral Health Group.

Helen V Worthington (HW): none known. HW is Co-ordinating Editor of the Cochrane Oral Health Group.

Ian Needleman (IN): none known. IN is an Editor with the Cochrane Oral Health Group.

Sarah H Wild (SW): none known.

David R Moles (DM): none known.

Brian Stevenson (BS): none known.

Susan Furness (SF): none known. SF is an Editor with the Cochrane Oral Health Group.

Zipporah Iheozor-Ejiofor (ZIE): none known. ZIE is salaried member of staff and an Editor with the Cochrane Oral Health Group.

\section{SOURCES OF SUPPORT}

\section{Internal sources}

- School of Dentistry, The University of Manchester, UK.

- Manchester Academic Health Sciences Centre (MAHSC), UK.

The Cochrane Oral Health Group is supported by MAHSC and the NIHR Manchester Biomedical Research Centre

\section{External sources}

- National Institute for Health Research (NIHR), UK.

This project was supported by the NIHR, via Cochrane Infrastructure funding to the Cochrane Oral Health Group. The views and opinions expressed therein are those of the authors and do not necessarily reflect those of the Systematic Reviews Programme, NIHR, NHS or the Department of Health

- Cochrane Oral Health Group Global Alliance, Other.

Through our Global Alliance (http://ohg.cochrane.org/partnerships-alliances), the Cochrane Oral Health Group has received support from: British Association for the Study of Community Dentistry, UK; British Association of Oral Surgeons, UK; British Orthodontic Society, UK; British Society of Paediatric Dentistry, UK; British Society of Periodontology, UK; Canadian Dental Hygienists Association, Canada; Mayo Clinic, USA; National Center for Dental Hygiene Research \& Practice, USA; New York University College of Dentistry, USA; and Royal College of Surgeons of Edinburgh, UK

\section{DIFFERENCES BETWEEN PROTOCOLANDREVIEW}

For the 2015 update the following amendments have been made to the published protocol for this review. 
- The original second objective (to identify whether further research is required in this area and if so, to identify the important research questions and appropriate study designs) and third objective (to investigate the various combinations of therapies used in treating periodontal disease in people with diabetes mellitus) have been removed as they are considered to be consequences of the outcome of the review.

- Periodontal treatment has been defined broadly to include any professionally-delivered intervention designed to reduce periodontal disease, and the criteria for types of interventions amended accordingly.

- Fructosamine has been deleted as an outcome measure because HbAlc is considered a more reliable and widely used measure of glycaemic control. Fructosamine (glycolated albumin) may be used as an indicator of glycaemic control over the previous 2 to 3 weeks in individuals who have atypical haemoglobin (eg sickle cell disease or thalassaemia), which does not form HbAlc.

- The previously vague secondary outcome 'oral hygiene' has been reworded as 'plaque indices.'

- Trials where participants have metabolic syndrome are specifically excluded from this review.

- Diagnostic assessment criteria for diabetes mellitus are now clearly stated.

- Periodontal outcome assessment was removed as a risk of bias domain, as it was agreed that the addition of periodontal outcome assessment misdirected attention from the primary focus (glycaemic control) of this review.

\section{N DEX TERMS}

\section{Medical Subject Headings (MeSH)}

Dental Scaling; Diabetes Mellitus, Type 1 [*blood]; Diabetes Mellitus, Type 2 [blood]; Glycated Hemoglobin A [metabolism]; Hyperglycemia [blood] [*therapy]; Oral Hygiene; Periodontal Diseases [blood] [therapy]; Randomized Controlled Trials as Topic; Root Planing; Time Factors

\section{MeSH check words}

Humans 